\title{
The distribution of dental features in non-avian theropod dinosaurs: Taxonomic potential, degree of homoplasy, and major evolutionary trends
}

\author{
Christophe Hendrickx, Octávio Mateus, Ricardo Araújo, and Jonah Choiniere
}

\begin{abstract}
Isolated theropod teeth are some of the most common fossils in the dinosaur fossil record and are continually reported in the literature. Recently developed quantitative methods have improved our ability to test the affinities of isolated teeth in a repeatable framework. But in most studies, teeth are diagnosed on qualitative characters. This can be problematic because the distribution of theropod dental characters is still poorly documented, and often restricted to one lineage. To help in the identification of isolated theropod teeth, and to more rigorously evaluate their taxonomic and phylogenetic potential, we evaluated dental features in two ways. We first analyzed the distribution of 34 qualitative dental characters in a broad sample of taxa. Functional properties for each dental feature were included to assess how functional similarity generates homoplasy. We then compiled a quantitative data matrix of 145 dental characters for 97 saurischian taxa. The latter was used to assess the degree of homoplasy of qualitative dental characters, address longstanding questions on the taxonomic and biostratigraphic value of theropod teeth, and explore the major evolutionary trends in the theropod dentition.

In smaller phylogenetic datasets for Theropoda, dental characters exhibit higher levels of homoplasy than non-dental characters, yet they still provide useful grouping information and optimize as local synapomorphies of smaller clades. In broader phylogenetic datasets, the degree of homoplasy displayed by dental and non-dental characters is not significantly different. Dental features on crown ornamentations, enamel texture and tooth microstructure have significantly less homoplasy than other dental features and can be used to identify many theropod taxa to 'family' or 'sub-family' level, and some taxa to genus or species. These features should, therefore, be a priority for investigations seeking to classify isolated teeth.

Our observations improve the taxonomic utility of theropod teeth and in some cases can help make isolated teeth useful as biostratigraphic markers. This proposed list of dental features in theropods should, therefore, facilitate future studies on the systematic paleontology of isolated teeth.
\end{abstract}

Hendrickx, Christophe, Mateus, Octávio, Araújo, Ricardo, and Choiniere, Jonah. 2019. The distribution of dental features in non-avian theropod dinosaurs: Taxonomic potential, degree of homoplasy, and major evolutionary trends. Palaeontologia Electronica 22.3.74 1110. https://doi.org/10.26879/820

palaeo-electronica.org/content/2019/2806-dental-features-in-theropods

Copyright: November 2019 Palaeontological Association.

This is an open access article distributed under the terms of Attribution-NonCommercial-ShareAlike 4.0 International (CC BY-NC-SA

4.0 ), which permits users to copy and redistribute the material in any medium or format, provided it is not used for commercial

purposes and the original author and source are credited, with indications if any changes are made.

creativecommons.org/licenses/by-nc-sa/4.0/ 
Christophe Hendrickx. Evolutionary Studies Institute, University of the Witwatersrand, South Africa. currently: Unidad Ejecutora Lillo, CONICET-Fundación Miguel Lillo, Miguel Lillo 251, San Miguel de Tucumán 4000, Tucumán, Argentina. christophendrickx@gmail.com Octávio Mateus. Museu da Lourinhã, 9 Rua João Luis de Moura, 2530-158, Lourinhã, Portugal; Universidade Nova de Lisboa, Departamento de Ciências da Terra, Faculdade de Ciências e Tecnologia, Quinta da Torre, 2829-516, Caparica, Portugal.omateus@fct.unl.pt Ricardo Araújo. Museu da Lourinhã, 9 Rua João Luis de Moura, 2530-158, Lourinhã, Portugal; Huffington Department of Earth Sciences, Southern Methodist University, PO Box 750395, 75275-0395, Dallas, Texas, USA; Instituto de Plasmas e Fusão Nuclear, Instituto Superior Técnico, Universidade de Lisboa, Portugal; Museum für Naturkunde, Berlin, Germany. rmaraujo@smu.edu Jonah Choiniere. Evolutionary Studies Institute, University of the Witwatersrand, South Africa. jonah.choiniere@wits.ac.za

Keywords: teeth; evolution; function

Submission: 22 September 2017. Acceptance: 23 October 2019.

\section{INTRODUCTION}

Theropods are a lineage of bipedal dinosaurs including birds and their most recent common ancestors (e.g., Padian and Chiappe, 1998; Chiappe and Witmer, 2002; Long and Schouten, 2008; Naish, 2012). Although non-avian theropods were mostly carnivores, there is abundant evidence for substantial trophic variation within the group, including herbivory (e.g., Kobayashi et al., 1999; Zanno et al., 2009; Sander et al., 2010; Zanno and Makovicky, 2011), omnivory (e.g., Holtz et al., 1998) and piscivory (e.g., Charig and Milner, 1997; Amiot et al., 2010; Xing et al., 2013b). This trophic diversity is reflected in a diverse array of tooth shape and dental morphologies within the group (Currie et al., 1990; Hendrickx and Mateus, 2014a; Hendrickx et al., 2015d).

Like fishes, crocodiles, squamates and other groups of dinosaurs, non-avian theropods are polyphyodont animals, i.e., they continuously replaced their teeth throughout their lifespan (Smith et al., 2005; Hendrickx et al., 2015d). Teeth, and particularly tooth enamel, are robust skeletal elements (Hillson, 2005), and most toothed theropods had 50 or more teeth that were replaced every one to two years (Fiorillo and Currie, 1994; Erickson, 1996). Consequently, theropod teeth are one of the most common fossils in terrestrial Mesozoic formations (e.g., Erickson, 1996; Smith et al., 2005; Blob and Badgley, 2007) and are constantly reported in the literature (e.g., Currie et al., 1990; Rauhut and Werner, 1995; Baszio, 1997; Zinke, 1998; Sankey et al., 2002; Sweetman, 2004; Maganuco et al., 2005; Vullo et al., 2007; Larson, 2008; Casal et al., 2009; Lubbe et al., 2009; Ösi et al., 2010; Han et al., 2011; Sues and Averianov, 2013; Larson and Currie, 2013; Richter et al., 2013; Torices et al., 2015; Kear et al., 2013; Madzia, 2014; Hendrickx and Mateus, 2014a; Cobos et al., 2014; Tavares et al., 2014; Fanti et al., 2014; Brusatte and Clark, 2015; Csiki-Sava et al., 2016; Gerke and Wings, 2016; Alonso et al., 2017; Malafaia et al., 2017a; Avrahami et al., 2018; Frederickson et al., 2018; Averianov et al., 2019; Wongko et al., 2019; Young et al., 2019).

Isolated theropod teeth provide taphonomic, paleoenvironmental and paleoecological data (e.g., Briggs and Crowther, 2001; Amiot et al., 2004, 2006, 2009, 2011; Rogers et al., 2007; Fanti et al., 2014; Gerke and Wings, 2016; Hassler et al., 2018; Frederickson et al., 2018). They may also provide evidence for paleodiversity, biostratigraphy (i.e., temporal/geographic ranges of theropod taxa), and anatomical information on clades when articulated skeletal fossils are missing or poorly represented (Brusatte et al., 2007; Larson et al., 2016). In addition, the size and morphology of theropod teeth and their denticles provide important functional, positional and trophic data that can be used to form hypotheses for body size, bite force and feeding behavior (e.g., D'Amore, 2009; D'Amore and Blumenschine, 2009; Reichel, 2010; Brink et al., 2015; Monfroy, 2017; Torices et al., 2018). Despite the importance of theropod teeth, their detailed morphology is often poorly known, leading to imprecise taxonomic assignments (e.g., Ösi et al., 2010; Amiot et al., 2011; Carrano et al., 2012; RuizOmeñaca et al., 2012; Torices et al., 2015; Madzia, 2014; Gerke and Wings, 2016). Such taxonomic imprecision obscures potentially useful information 
that teeth may provide for paleogeographic and stratigraphic distributions of theropod clades.

Morphometric multivariate analyses have shown promise for identifying isolated teeth (e.g., Smith et al., 2005; Larson and Currie, 2013) and recent studies have indicated that qualitative characters can also be useful for differentiating taxa, even for theropods with similar dentition (Hendrickx and Mateus, 2014a; Hendrickx et al., 2015c). Relatively few theropods have had their dentition studied in detail, with notable exceptions of Coelophysis (Buckley and Currie, 2014), Majungasaurus (Fanti and Therrien, 2007; Smith, 2007), megalosaurids (Hendrickx et al., 2015c), Albertosaurus (Buckley et al., 2010), Tyrannosaurus (Smith, 2005), Troodon (Currie, 1987) and Buitreraptor (Gianechini et al., 2011b). Recent research has shown that much variation in teeth remains to be described, particularly with respect to denticle shape, cross-sectional geometry, extension of the mesial carina and the presence of crown ornamentations.

The scarcity of information on theropod tooth morphology leads to taxonomic assignments on the basis of a priori assumptions of their phylogenetic affinities (Smith, 2005). The current literature contains many tooth-based taxonomic assessments based on scarce or poorly understood data (e.g., Soto and Perea, 2008; Buffetaut, 2011; Vullo et al., 2014; Mo and Xu, 2015; Serrano-Martínez et al., 2015, 2016). For example, the marginal undulations visible on the crown of some carcharodontosaurids is often considered as a key character of this clade, leading some authors (e.g., Chure et al., 1999) to assign isolated teeth to Carcharodontosauridae solely based on this feature when, in fact, it has a broader taxonomic distribution (Brusatte et al., 2007). Another example is the presence of mesial denticles significantly smaller than the distal denticles, a dental feature long thought to characterize dromaeosaurid teeth (e.g., Rauhut and Werner, 1995; Milner, 2002; Sweetman, 2004) but also seen on the crowns of other distantly related clades (Rauhut et al., 2010). A broader, more comprehensive understanding of the distribution of key dental morphologies in theropods will reduce errors in taxonomic assessments of isolated teeth.

This research investigates the distribution of 34 discrete dental characters in 200 saurischians, mostly non-avian theropods, and provides functional properties for each of them. We then use a data matrix of 145 dentition-based characters coded in 97 saurischian taxa and a variety of trees from the literature to map the distribution of these characters and to assist other systematists in developing dental character sets. Using our character distributions, we evaluate the taxonomic value of theropod teeth and propose dentitionbased synapomorphies for several theropod lineages. We also evaluate homoplasy in different partitions within dental characters and compare our assessments to homoplasy levels in character sets derived from the rest of the skeleton. Finally, we explore the major transformations occurring in the dentition of non-avian theropods throughout their evolution. Our findings should be of broad use to improve the accuracy of taxonomic assessment of isolated theropod teeth, facilitate future study on the systematic paleontology of isolated theropod teeth and ultimately to improve their potential as biostratigraphic markers.

\section{MATERIALS AND METHODS}

\section{Material}

We investigated dental features on teeth preserved within the upper and lower jaws as well as isolated teeth belonging to a total of 198 taxa bracketed phylogenetically between the basal saurischian Herrerasaurus ischigualastensis (Reig, 1963; Sereno and Novas, 1994) and the basal avialan Archaeopteryx lithographica (Godefroit et al., 2013a; Foth et al., 2014; Lefèvre et al., 2017; Appendix 1.1). The basal saurischians Daemonosaurus (Sues et al., 2011), Eodromaeus (Martínez et al., 2011) and Eoraptor (Sereno et al., 1993, 2013), as well as the Scansoriopterygidae (Czerkas and Yuan, 2002) and the Anchiornithinae (sensu Xu et al., 2016; Agnolin et al., 2019; 'Anchiornithidae' of Foth and Rauhut, 2017 and 'Anchiorninae' of Hu et al., 2018), recently recovered as non-avialan theropods by some authors (Sues et al., 2011; Brusatte et al., 2014; Xu et al., 2015b, 2017; Baron et al., 2017; Cau et al., 2017; Müller et al., 2018; Hu et al., 2018), were also included in this study. Of these 200 taxa, we examined firsthand the dentition of 125 taxa deposited in 35 scientific collections from Argentina, France, Belgium, Germany, Italy, Spain, Portugal, Qatar, Switzerland, the United Kingdom, South Africa, China, Canada and the United States (Appendix 1.1). Anatomical observations were assisted with the use of an AM411T Dino-Lite Pro digital microscope. The dentition of a further 34 non-avian theropod taxa was examined from high-quality casts and high-resolution photographs provided by colleagues. Publications with well-illustrated and/or 
well-described teeth were used to study an additional 41 taxa (Appendix 1.1).

\section{Nomenclature on the Theropod Dentition and Theropod Classification}

The anatomical, positional, directional and morphometric nomenclature used in this study (Figure 1) follows the terminology proposed by Hendrickx et al. (2015d). This terminology is partly based on the measurements provided by Smith et al. (2005) and the directional terminology defined by Smith and Dodson (2003). We also follow the dental notation proposed by Smith and Dodson
(2003), which identifies the side of the jaw (i.e., left $=\mathrm{L}$; right $=\mathrm{R}$ ), followed by the abbreviation of the tooth-bearing bone (i.e., premaxilla $=\mathrm{pm}$; maxilla $=$ $\mathrm{mx}$; dentary $=\mathrm{dt}$ ) and then the position occupied along the tooth-bearing bone (e.g., Rmx3 for the third right maxillary tooth). When referred to cranial and mandibular bones, the non-standardized traditional Owenian/Romerian directional and anatomical terms (Harris, 2004; Wilson, 2006) were favored over the terminology of the Nomina Anatomica Veterinaria (ICVGAN, 2012) and the Nomina Anatomica Avium (Baumel, 1993), because they are the most commonly used in the
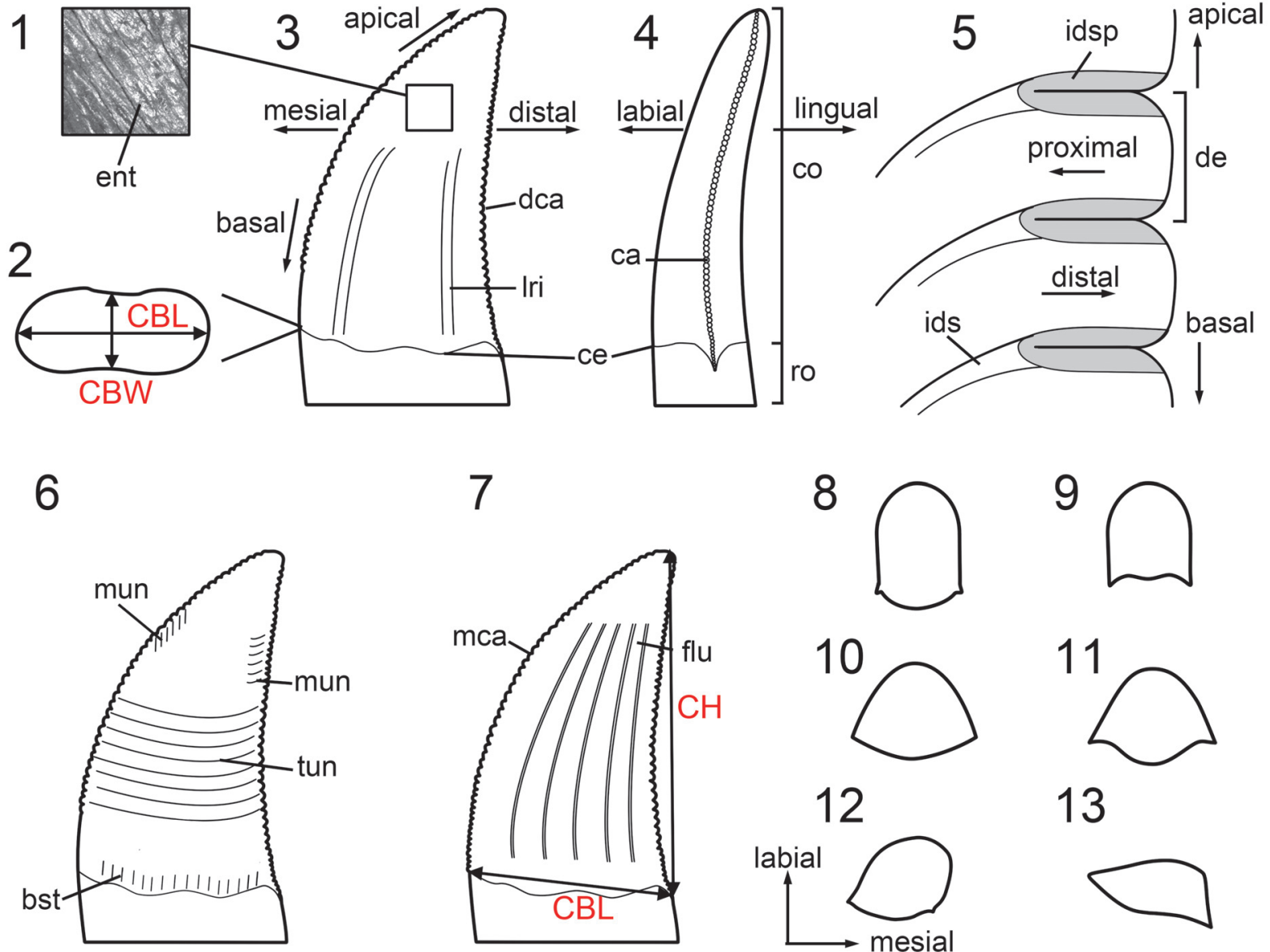

8
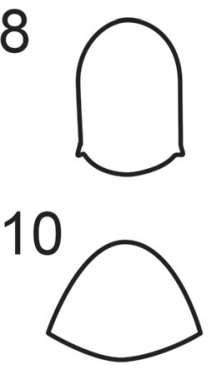

12

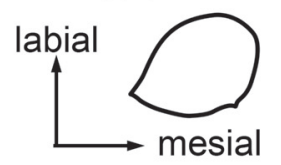

9

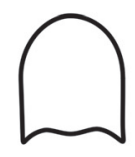

11

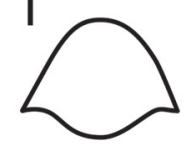

13

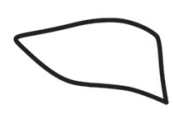

FIGURE 1. Anatomical, directional, and morphometric terminology used in this study. Figure modified from Hendrickx et al. (2015d). 1, Close up of the enamel surface of crown in 3 in labial view; 2, basal cross-section of crown in 3 in basal view showing CBL (crown-base length) and CBW (crown-base width); $\mathbf{3}$, idealized lateral theropod tooth in labial view; 4, idealized lateral theropod tooth in distal view; 5 , idealized denticles of a denticulated distal carina in labial view; 6, idealized lateral theropod tooth in labial view showing the crown ornamentations; 7, idealized fluted theropod tooth in labial view showing $\mathrm{CBL}$ and $\mathrm{CH}$ (crown height); 8, U-shaped cross-section with convex lingual margin; 9, U-shaped cross-section with central ridge on the lingual margin; 10, D-shaped cross-section; 11, salinonshaped cross-section; 12, labiolingually wide J-shaped cross-section; 13, labiolingually narrow J-shaped cross-section. Abbreviations: bst, basal striation; ca, carina; ce, cervix; co, crown; dca, distal carina; de, denticle; ent, enamel texture; flu, flute; ids, interdenticular sulcus; idsp, interdenticular space; Iri, longitudinal ridge; mun, marginal undulation; mca, mesial carina; ro, root; tun, transverse undulation. 
non-avian theropod literature (Eddy and Clarke, 2011; Hendrickx and Mateus, 2014b; Hendrickx et al., 2015a). Consequently, 'anterior' and 'posterior' are used as directional terms rather than the veterinarian alternatives 'cranial' and 'caudal', respectively.

The theropod phylogeny used to investigate the distribution of dental features in non-avian theropods mostly follows the classification recently summarized by Hendrickx et al. (2015b), with variation in the phylogenetic position of Eoraptor, Eodromaeus, Daemonosaurus, Dracovenator, Limusaurus and Megaraptora. The classification here-followed is based on the results obtained by: Müller et al. (2018, fifth phylogenetic analysis), based on the dataset of Langer et al. (2017), for non-neotheropod Saurischia; Ezcurra (2017) and Wang et al. (2017a) for non-averostran Neotheropoda; Rauhut and Carrano (2016) and Wang et al. (2017a) for Ceratosauria; Carrano et al. (2012) and Rauhut et al. (2016) for non-coelurosaurian Tetanurae; Arden et al. (2019) for Spinosauridae; and Brusatte and Carr (2016) and Delcourt and Grillo (2018) for Tyrannosauroidea, with Pantyrannosauria as the sister clade of Proceratosauridae (Figure 2). We also follow the phylogenetic tree obtained by Cau et al. (2017) based on the dataset of Brusatte et al. (2014) for non-tyrannosauroid Coelurosauria, with Anchiornithinae being recovered as a basal clade among Troodontidae. We follow Ezcurra's (2017) nomenclature for 'Syntarsus' kayentakatae and Megapnosaurus rhodesiensis, also known as Coelophysis kayentakatae and Coelophysis rhodesiensis (e.g., Nesbitt and Ezcurra, 2015; Martínez and Apaldetti, 2017; Piechowski et al., 2019), based on the phylogenetic distribution of these two distantly related taxa among Coelophysoidea. The phylogenetic placement of Sciurumimus at the base of the megalosaurid clade follows the result of the cladistic analysis obtained by Rauhut et al. (2012). Aorun was recently suggested to be the basalmost Alvarezsauroidea by Xu et al. (2018), a paper which came out at the final stage of the correction of this manuscript. The dental evolution of theropods is, therefore, explored based on the phylogenetic placement of Aorun at the base of Coelurosauria. We, however, briefly discuss the evolution of the dentition in Maniraptoriformes based on the inclusion of this taxon within Alvarezsauroidea. We finally adopt the phylogenetic definitions compiled by Hendrickx et al. (2015b) and Hendrickx and Carrano (2016) for each of the non-avian theropod clades, with the exception of Abelisauridae (here being defined as the most inclusive clade containing Carnotaurus sastrei but not Ceratosaurus nasicornis and Noasaurus leali) and Caenagnathoidea (here defined as the least inclusive clade containing Avimimus portentosus, Oviraptor philoceratops and Caenagnathus collinsi).

In most cladistic analyses recently performed on Coelurosauria, Compsognathidae are more closely related to Maniraptoriformes than to Tyrannosauroidea (e.g., Senter, 2007, 2011; Smith et al., 2008; Dal Sasso and Maganuco, 2011; Carrano et al., 2012; Turner et al., 2012; Choiniere et al., 2014a; Brusatte et al., 2014), and the name Neocoelurosauria (Hendrickx, 2015; Paul, 2016) is here used to refer to the clade Compsognathidae + Maniraptoriformes. Likewise, the clade Troodontinae (Gilmore, 1924), defined by Martyniuk (2012, p. 178) as "<Troodon formosus \& Saurornithoides mongoliensis", or the least inclusive clade containing Troodon formosus and Saurornithoides mongoliensis, here corresponds to the least inclusive clade containing Sinovenator changii and Troodon formosus, most members of which have teeth bearing denticles. Finally, as for the clade Caenagnathoidea (i.e., Avimimus + Caenagnathidae + Oviraptoridae; sensu Qiu et al., 2019) which gathers edentulous taxa, the clade Ornithomimoidea here refers to toothless ornithomimosaurs, which includes the 'family'-level clades Ornithomimidae and Deinocheiridae (sensu Lee et al., 2014b). This node-based taxon can be defined as the least inclusive clade containing Ornithomimus velox and Deinocheirus mirificus (for another definition, see Sereno, 2017).

\section{Distribution and Degree of Homoplasy of Dental Features}

The distribution, degree of homoplasy and phylogenetic utility of qualitative dental characters were assessed by using a modified version of the dentition-based data matrix created by Hendrickx and Mateus (2014a; Appendices 2, 3.1 and 3.2). To this original dataset of 60 taxa, we removed the poorly known paravian Richardoestesia gilmorei (Currie et al., 1990) and added 38 new theropod taxa, especially focusing on those with peculiar dentition [e.g., Chilesaurus (Novas et al., 2015); Halszkaraptor (Cau et al., 2017); Limusaurus (Wang et al., 2017a)] and basally branching members of major theropod clades (e.g., Nqwebasaurus; De Klerk et al., 2000; Choiniere et al., 2012). Herrerasaurus ischigualastensis (Reig, 1963; Sereno and Novas, 1994) and Archaeopteryx lithographica (Meyer, 1861; Howgate, 1984; Rauhut, 


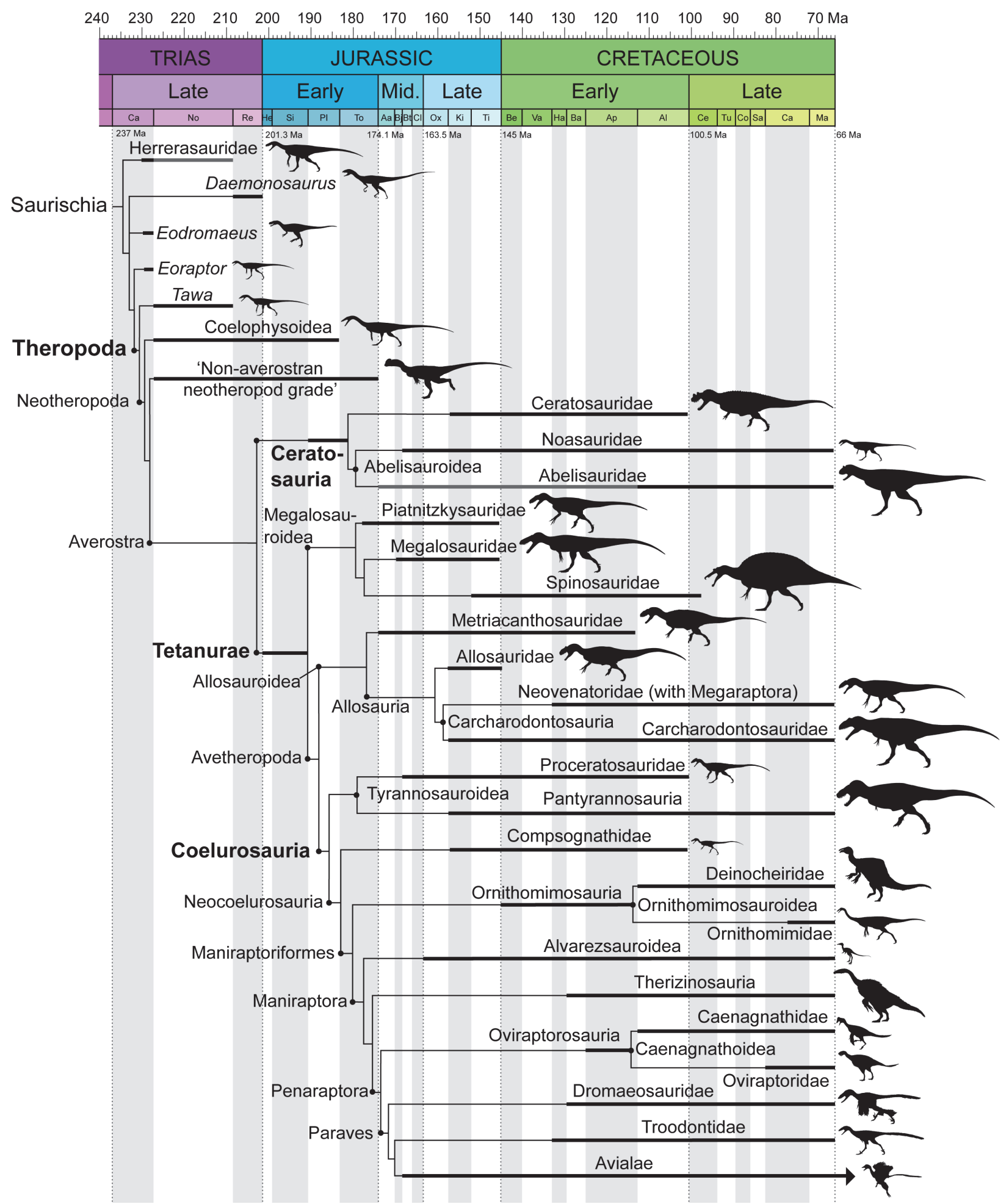

FIGURE 2. Phylogeny and stratigraphic distribution of theropod dinosaurs. Figure modified from Hendrickx et al. (2015b; see references therein). The theropod silhouettes are from Conty (Eodromaeus), the Smithsonian Institution (Daemonosaurus; modified), Julio Garza (Dilophosauridae), Gregory S. Paul (Metriacanthosauridae), T. Michael Keesey (Deinocheiridae), Funkmonk (Alvarezsauroidea and Therizinosauria), Jaime Headden (Caenagnathidae), and Scott Hartman (all other silhouettes). All silhouettes other than those from the Smithsonian Institution and Gregory S. Paul were downloaded from phylopic.org and are under a Creative Commons Attribution-NonCommercial-ShareAlike 3.0 Unported License unless stated otherwise (see Appendix 1.2). 
2014) were used to bracket the sample of interest phylogenetically. We added several edentulous (e.g., adult Limusaurus, Citipati, and Struthiomimus) and partially edentulous taxa (e.g., Erlikosaurus, juvenile Limusaurus, Shenzhousaurus) to this matrix. We also revised a large number of the dental characters used in that study from personal observation of the specimens and changes made by Gerke and Wings (2016; see Appendix 3.4 and 3.5). Characters 30,49 and 73 were deleted (characters 71 and 73 were combined) and seven new dental features (char. 17, 18, 27, 35, 36, 54, and 145) were added. In total, our revised data matrix comprises 145 characters (Appendix 2) coded for a total of 97 saurischian taxa (Appendix 3.1 and 3.2). Distribution of dental features. The distribution of dental characters was visualized on eight trees representative of alternative phylogenetic hypotheses for non-avian theropod evolution. Trees were built using Mesquite 3.2 (Maddison and Maddison, 2017). Variations in the topology result from the differing placement of: 1) ceratosaurids outside abelisauroids (Rauhut and Carrano, 2016) or among non-noasaurid ceratosaurs (Wang et al., 2017a); 2) Monolophosaurus as a megalosauroid (Rauhut et al., 2016) or a non-orionide tetanuran (Carrano et al., 2012); 3) megaraptorans among neovenatorids (Carrano et al., 2012) or tyrannosauroids (Porfiri et al., 2014); 4) Epidexipteryx within Avialae (Foth and Rauhut, 2017) or Oviraptorosauria (Brusatte et al., 2014); 5) Troodontidae as a sister clade of Avialae (Cau et al., 2017; Foth and Rauhut, 2017) or Dromaeosauridae and, therefore, forming the clade Deinonychosauria (Turner et al., 2012); 6) Eshanosaurus as the basalmost (Xu et al., 2001) or a more derived member of Therizinosauria (Barrett, 2009); 7) Scipionyx as a compsognathid (Dal Sasso and Maganuco, 2011) or a closely related taxon of Ornitholestes (Choiniere et al., 2014a). Character distributions for dental features were visualized on each tree using TNT 1.5 (Goloboff and Catalano, 2016) and WinClada 1.00.08 (Nixon, 2002) based on the Nexus file created with Mesquite 3.2.

Isolated shed teeth are typically the most common theropod material found on dinosaur fossil sites. We, therefore, examined the distribution of crown-based characters (i.e., characters on mesial and lateral crowns) as well as enamel surface texture (i.e., characters 38 to 121). Likewise, given that the majority of theropod shed teeth belong to the lateral dentition (i.e., maxillary and dentary teeth that differ significantly in their morphology from that of premaxillary and mesial dentary teeth;
Hendrickx et al. 2015c), the distribution of characters on the lateral dentition (i.e., characters 67 to 121) was also examined.

Degree of homoplasy of dental characters. Several methods were performed to evaluate the role of dentition-based characters in phylogenetic reconstruction and to measure the degree of homoplasy of dental features.

- To assess the amount of homoplasy in dental characters and to quantify their utility in providing grouping information, we employed the ensemble consistency and retention indices $(\mathrm{Cl}$ and $\mathrm{RI}$, respectively; Kluge and Farris, 1969; Farris, 1989) and the individual character consistency and retention indices (ci and ri, respectively). $\mathrm{Cl}$ is a measure of the amount of homoplasy of an entire dataset on a tree, whereas ci measures the amount of homoplasy in a given character on this tree (Kitching et al., 1998) $\mathrm{Cl}$ and ci range from 1 (i.e., a $\mathrm{Cl}$ of 1 denotes that there is no homoplasy and a ci of 1 that the character is non-homoplastic) to a value that asymptotically approaches 0 with increasing amounts of homoplasy, and, for $\mathrm{Cl}$, with increasing character sample size. Conversely, RI measures the amount of homoplasy retained as a local synapomorphy (i.e., meaning how many of the homoplastic characters still convey some grouping information) of an entire dataset for a tree, whereas ri measures the amount of homoplasy retained as a local synapomorphy for a given character on this tree. RI and ri range in value from 1 (i.e., all characters fit the tree perfectly; non-ambiguous synapomorphy) to 0 (i.e., no character is synapomorphic for a certain clade; nonsynapomorphic character). We also used $\mathrm{Cl}$ and $\mathrm{RI}$ for mean values of ci and ri that were calculated for a set of characters related to the same dentition sub-unit. To provide a baseline assessment of homoplasy, we calculated $\mathrm{Cl}$ and $\mathrm{RI}$ in all eight trees of the non-avian theropod classification. The 145 dental characters and the stats.run script available in TNT (http://phylo.wikidot.com/ tntwiki) were used to determine the most consistent tree topology in terms of dental features. We calculated $\mathrm{Cl}$ and $\mathrm{RI}$ in datasets restricted to non-averostran Saurischia (9 taxa), Ceratosauria (14 taxa), Megalosauroidea (14 taxa), Allosauroidea (13 taxa), Coelurosauria (47 taxa), Maniraptoriformes 
(35 taxa), Paravians (19 taxa) and Dromaeosauridae (11 taxa) to determine the theropod lineages with lowest homoplasy levels in terms of dental characters and to quantify the utility of dental characters for grouping information. Uninformative characters were deactivated using the command XINACT before calculating $\mathrm{Cl}$ and $\mathrm{RI}$ values for each topological tree and each clade.

- To assess and compare the degree of homoplasy between dental and non-dental characters, we appended our dentitionbased characters to eight of the most recent datasets on non-avian theropods [i.e., Lee et al. (2014a) based on Cau et al.'s (2014) data matrix, Choiniere et al. (2014a) and Wang et al. (2017a) for non-avian Theropoda; Tortosa et al. (2014) and Rauhut and Carrano (2016) for Ceratosauria; Carrano et al. (2012) for non-coelurosaur Tetanurae; and Cau et al. (2015) using Brusatte et al.'s (2014) dataset and Foth and Rauhut (2017) for Coelurosauria; see Appendix 3.6], by firstly removing the preexisting dental characters from each dataset. All eight resulting supermatrices were imported to Mesquite 3.2 (Maddison and Maddison, 2017) and the tree topology obtained by the authors of each of these data matrices was built with the same software. The $\mathrm{Cl}$ and $\mathrm{RI}$ were calculated on these trees using TNT for each resulting supermatrix after all uninformative characters were deactivated and when the dental characters were: i) included in the dataset, ii) excluded from the supermatrix, and iii) considered separately (i.e., all nondental characters were excluded from the dataset).

- We then calculated the consistency (ci) and retention (ri) indexes for all dental and nondental characters in all five supermatrices using the TNT scrip CharStats.run (Ramírez, 2013) and performed a one-sample t-test and Mann-Whitney U-test to ascertain if there were statistically significant differences between the mean values of ci (here noted $\mathrm{Cl}$ ) and ri (here noted RI) of dental and nondental characters. These statistics, which are parametric and non-parametric estimators of differences in mean between two sets of characters, were performed in all eight supermatrices using PAST3 (Hammer et al., 2001), and the corresponding $p$-values and
Mann-Whitney-scores are found in Appendix 4.1 and 4.2 .

- To know whether particular partitions of the dentition and tooth are more reliable than others, we calculated ci and ri for each of the 145 dental characters in all eight theropod tree topologies and measured the mean value of ci and ri (here noted $\mathrm{Cl}$ and $\mathrm{RI}$, respectively) for each dentition sub-unit, which we arbitrarily pre-defined (i.e., premaxillary, maxillary, dentary and palatal teeth, crowns, carinae, denticles and ornamentations for the mesial and lateral dentition, enamel texture and microstructure, and root). We then performed an ANOVA test to identify differences in variance between dentition sub-units using the ci and ri values of all 145 dental characters in the most consistent topological tree. To make post hoc identifications, of which sub-units varied significantly, we calculated Mann-Whitney pairwise comparisons using Bonferroni-corrected $p$-values on the ANOVA data using PAST3 (Appendix 4.1 and 4.2).

\section{Institutional Abbreviations}

AM, Albany Museum, Grahamstown, South Africa; AMNH, American Museum of Natural History, New York City, USA; ANSP, Academy of Natural Sciences, Philadelphia, Pennsylvania, USA; AODF, Australian Age of Dinosaurs Fossil, Australian Age of Dinosaur Museum of Natural History, Winton, Queensland, Australia; BMMS, Bürgermeister Müller Museum, Solnhofen, Germany; BMNHC, Beijing Museum of Natural History, Beijing, China; BP, Evolutionary Studies Institute (formerly "Bernard Price Institute for Palaeontological Research"), University of the Witwatersrand, Johannesburg, South Africa; BSPG, Bayerische Staatssammlung für Paläontologie und Historische Geologie, München, Germany; BYU-VP, Brigham Young University Museum of Vertebrate Paleontology, Provo, USA; CAGS, Chinese Academy of Geological Sciences, Beijing, China; CCMGE, Chernyshev's Central Museum of Geological Exploration, Saint Petersburg, Russia; CEU, College of Eastern Utah, Price, Utah, USA; CMNH, Carnegie Museum of Natural History, Pittsburgh, USA; CV, Chongqing Museum of Natural History, Chongqing, China; DINO, Dinosaur National Monument, Vernal, Utah, USA; DLXH, Dalian Xinghai Museum, Dalian, Liaoning Province, China; DMNH, Perot Museum of Nature and Science, Dallas, Texas, USA; DMNS, Denver Museum of 
Nature and Science, Denver, Colorado, USA; DMR-TF, Department of the Mineral Resources, Palaeontological collection, Bangkok, Thailand; DNHM, Dalian Natural History Museum, Dalian, Liaoning Province, China; ELDM, Erenhot Dinosaur Museum, Erenhot, Inner Mongolia, China; FMNH, Field Museum of Natural History, Chicago, USA; FPDM, Fukui Prefectural Dinosaur Museum, Katsuyama, Fukui, Japan; FRDC-GS, Fossil Research and Development Center, Gansu Bureau of Geology and Mineral Resources Exploration, Lanzhou, China; GR, Ghost Ranch Ruth Hall Museum of Paleontology collections, Ghost Ranch Conference Center, New Mexico, USA; HG, Paleontological Center, Bohai University, Jinzhou City, China; HGM, Henan Geological Museum, Zhengzhou, Henan Province, China; HMN, Museum für Naturkunde, Berlin, Germany; ISIR, Indian Statistical Institute, Kolkata, India; IVPP, Institute for Vertebrate Paleontology and Paleoanthropology, Beijing, China; JLUM, Geological Museum of the Jilin University, Changchun, Jilin province, China; JME, Jura Museum Eichstätt, Eichstätt, Germany; JMP, Jinzhou Museum of Paleontology, Jinzhou, Liaoning Province, China; JZMP, Jinzhou Museum of Paleontology, Jinzhou, Liaoning Province, China; KMV, Kunming Municipal Museum, Guandu district, China; LDM-LCA, Lufeng Dinosaur Museum-Lufeng Chuanjie A'na, A'na, China; LFGT, Bureau of Land and Resources of Lufeng County, Lufeng, Yunnan, China; LH PV, Long Hao Institute of Geology and Paleontology, Hohhot, Nei Mongol, China; LHC, Las Hoyas Collection, Universidad Autónoma de Madrid, Madrid, Spain; LPMB, Liaoning Paleontological Museum, Liaoning, China; MACN, Museo Argentino de Ciencias Naturales 'Bernardino Rivadavia,' Buenos Aires, Argentina; MB, Museum für Naturkunde der Humboldt Universität, Berlin, Germany; MCCM, Museo de Ciencias de Castilla-La Mancha [now MUPA, Museo de Paleontología de Castilla-La Mancha], Cuenca, Spain; MCF-PVPH, Museo Municipal 'Carmen Fuñes,' Plaza Huincul, Argentina; MCZ, Museum of Comparative Zoology, Harvard University, Cambridge, Massachusetts, USA; MG, Museu Geológico, Lisbon, Portugal; MHNA-PV, Muséum d'Histoire Naturelle d'Aix-en-Provence, France; MIWG, Dinosaur Isle, Isle of Wight Museum Services, Sandown, UK; ML, Museu da Lourinhã, Lourinhã, Portugal; MLP, Museo de La Plata, La Plata, Argentina; MMCN-PV, Museo Municipal 'Ernesto Bachmann,' Villa El Chocón, Neuquén, Argentina; MML, Museo Municipal de Lamarque, Río Negro, Argentina; MNA, Museum of Northern
Arizona, Flagstaff, Arizona, USA; MNHN, Muséum national d'Histoire naturelle, Paris, France; MNN, Musée National du Niger, Niamey, Niger; MNUFR, Mongolia National University, Ulaanbaatar, Mongolia; MOR, Museum of the Rockies, Bozeman, Montana, USA; MPCA, Museo Provincial Carlos Ameghino, Cipolletti, Río Negro, Argentina; MPCD, Institute of Paleontology and Geology, Mongolian Academy of Sciences (formerly IGM), Ulaanbaatar, Mongolia; MPCO.V, Museu de Paleontologia de Cruzeiro do Oeste, Cruzeiro do Oeste, Brazil; MPEF-PV, Museo Paleontológico 'Egidio Feruglio,' Trelew, Argentina; MPM-Pv, Museo Padre Molina Paleontología de Vertebrados, Río Gallegos, Santa Cruz, Argentina; MSM, Mesa Southwest Museum, Mesa, Arizona, USA; MSNM, Museo di Storia Naturale di Milano, Milan, Italy; MSP, Arizona Museum of Natural History, Mesa, Arizona, USA; MUCPv, Museo de la Universidad Nacional del Comahue, Neuquén, Argentina; MWC, Museum of Western Colorado, Fruita, Colorado, USA; NCSM, North Carolina Museum of Natural Sciences, Raleigh, USA; NGMC, National Geological Museum of China, Beijing, China; NHFO, Natural History Fossil Collection, Qatar Museum Authority, Doha, Qatar; NHMUK PV, Natural History Museum, London, UK; NIGP, Nanjing Institute of Geology and Palaeontology, Nanjing, China; NMC, Canadian Museum of Nature, Ottawa, Ontario, Canada; NMMNH, New Mexico Museum of Natural History and Science, Albuquerque, New Mexico, US; NMW, National Museum Wales, Cardiff, UK; OCP, Office Chérifien des Phosphates, Khouribga, Morocco; OUMNH, Oxford University Museum, Oxford, UK; PIN, Paleontological Institute, Russian Academy of Sciences, Moscow, Russia; PMoL, Paleontological Museum of Liaoning, Shenyang, Liaoning, China; PULR, Paleontología, Universidad Nacional de La Rioja, La Rioja, Argentina; PVL, Fundación 'Miguel Lillo,' San Miguel de Tucumán, Argentina; PVSJ, Museo de Ciencias Naturales, Universidad Nacional de San Juan, San Juan, Argentina; QG, Zimbabwe Natural History Museum, Bulawayo, Zimbabwe; RMM, McWane Science Center, Birmingham, Alabama, USA; ROM, Museum of the Rockies, Bozeman, Montana, USA; RTMP, Royal Tyrrell Museum of Palaeontology, Drumheller, Alberta, Canada; SBA-SA, Soprintendenza per i Beni Archeologici di Salerno Avellino Benevento e Caserta, Salerno, Italia; SGM, Ministère de l'Énergie et des Mines, Rabat, Morocco; SMA, Sauriermuseum Aathal, Aathal, Switzerland; SMNS, Staatliches Museum für Naturkunde, Stuttgart, 
Germany; SMU, Southern Methodist, University, Dallas, USA; SNGM, Servicio Nacional de Geología y Minería, Santiago, Chile; STM, Shandong Tianyu Museum of Nature, Pingyi, Shandong Province, China; TPII, Thanksgiving Point Institute, Inc., North American Museum of Ancient Life, Lehi, Utah, USA; UA, Université d'Antananarivo, Antananarivo, Madagascar; UC, University of Chicago Paleontological Collection, Chicago, USA; UCM, University of Colorado Museum of Natural History, Boulder, Colorado, USA; UCMP, University of California Museum of Paleontology, Berkeley, USA; UMNH, Natural History Museum of Utah, University of Utah, Salt Lake City, USA; USNM, United States National Museum Vertebrate Paleontology, National Museum of Natural History, Washington, District of Columbia, USA; USP, Universidade de São Paulo, São Paulo, Brazil; WDC, Wyoming Dinosaur Center, Thermopolis, Wyoming, USA; WMN, LWL-Museum für Naturkunde, Münster, Germany; YFGP, Yizhou Fossil and Geology Park, Liaoning, China; YPM, Yale Peabody Museum of Natural History, Yale, Connecticut, USA; ZCDM, Zhucheng Dinosaur Museum, Zhucheng, China; ZDM, Zigong Dinosaurian Museum, Zigong, Sichuan, China; ZIN PH, Paleoherpetological Collection, Zoological Institute, Russian Academy of Sciences, Saint Petersburg, Russia; ZLJT, Lufeng World Dinosaur Valley Park, Yunnan, China; ZPAL, Institute of Palaeobiology of the Polish Academy of Sciences, Warsaw, Poland.

\section{RESULTS}

\section{Summary}

- 'Super'-family and 'family'-level clades are better defined by dental characters than major theropod clades such as Ceratosauria, Maniraptora and Tetanurae.

- Spinosauridae (21 synapomorphies), followed by Allosauroidea (7), and Abelisauroidea, Tyrannosauroidea and Therizinosauria (6), are the best-supported clades in terms of dental features.

- $\mathrm{Cl}$ values of the dentition-based data matrix are particularly low $(\sim 0.2)$ in all eight trees of the theropod phylogeny, and $\mathrm{RI}$ values are close to 0.45 .

- The tree topology hypothesizing Epidexipteryx as the basalmost oviraptorosaur is the most consistent in terms of dental features.

- Megalosauroidea is the theropod clade with the least amount of dental homoplasy, and whose dental characters provide the most useful grouping information.

- Excluding dental characters improves $\mathrm{Cl}$ and $\mathrm{RI}$ in all eight supermatrices (i.e., character matrices that combine our dentitionbased data matrix with different recently published matrices on the theropod skeleton).

- $\mathrm{Cl}$ values are not significantly different when including or excluding dental characters in Choiniere et al.'s (2014a) and Lee et al.'s (2014a) matrices dealing with non-avian theropod classification.

- Highest $\mathrm{Cl}$ values of dental characters are obtained for the datasets of Tortosa et al. (2014) and Rauhut and Carrano (2016) on ceratosaur relationships.

- Among all dental sub-units, the crown enamel texture and microstructure show the highest $\mathrm{Cl}$ and $\mathrm{RI}$ values.

\section{Distribution of Apomorphic Dental Characters}

Our sample of eight trees shows that the majority of theropod clades and OTUs are diagnosed by dentition-based characters that show homoplasy across our taxonomic sample (Figures 3 and 4; Appendices 3.1 and 5). In non-avetheropod theropods, most major clades such as Neotheropoda, Averostra, Ceratosauria, Tetanurae, Orionides and Megalosauroidea are defined by zero-to-two dentition-based synapomorphies, whereas three or more dental features support most 'family' -level clades such as Ceratosauridae, Abelisauridae, Piatnitzkysauridae and Spinosauridae. In Avetheropoda, most major clades and 'super-family' and 'family'-level subclades such as Avetheropoda, Allosauroidea, Neovenatoridae, Carcharodontosauridae, Coelurosauria, Tyrannosauroidea, Compsognathidae, Ornithomimosauria, Therizinosauria, Maniraptoriformes, Pennaraptora, Dromaeosauridae and Troodontidae are also supported by three or more dentitionbased synapomorphies. The distribution of crownbased characters (i.e., characters related to the mesial and lateral crowns and excluding those on the size, disposition and outline of premaxillary, maxillary and dentary alveoli as well as the root and enamel microstructure) also shows that many 'super-family', 'family' or 'sub-family' level clades are defined by a combination of three or more crown-based characters (Appendices 3.1 and 5, Tree topology 9). Likewise, the majority of theropod genera are diagnosed by two or more crown-based autapomorphies, particularly so in non-maniraptori- 


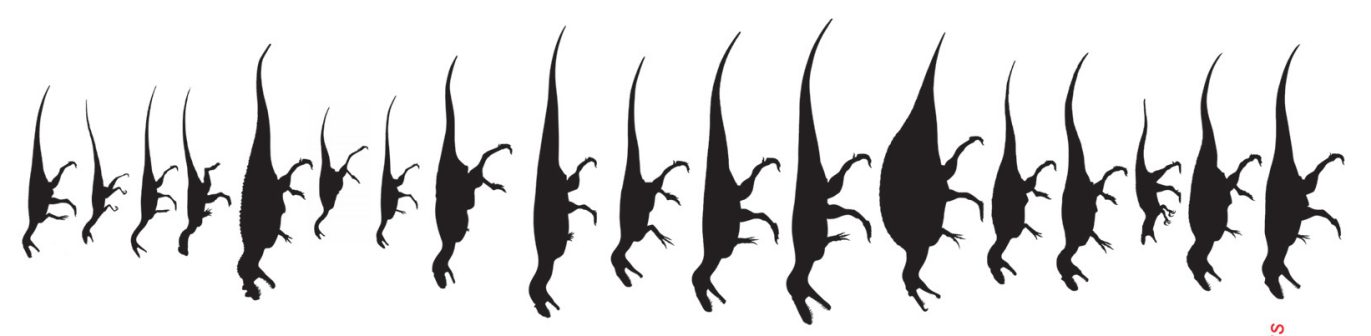

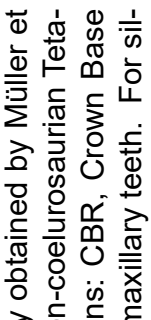

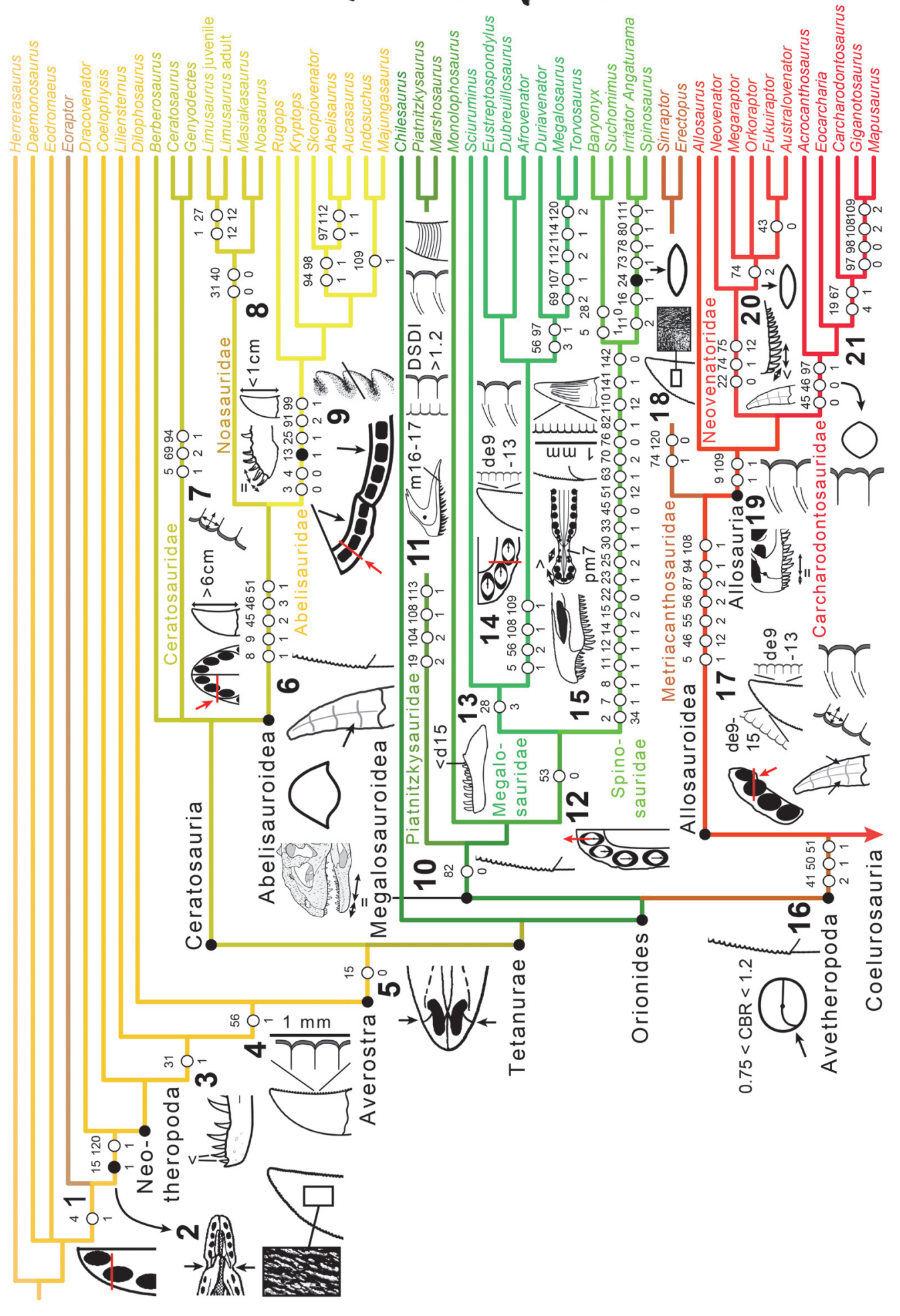

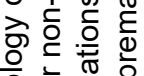
흥 훙 응

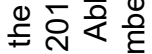

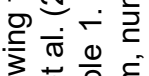

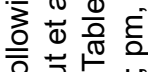
는 록 ब 정 응 든 흥 흘 ڤ) 은 Ф్

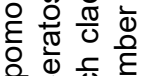
造造 है के 흔 选

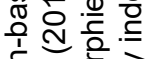
을 市 ฮ

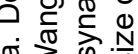

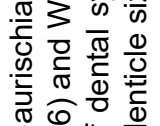
ஸ

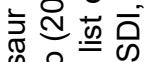

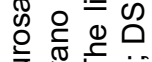
至 ○ல ळ

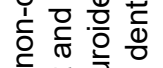

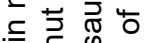
क

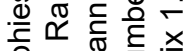

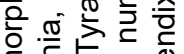
है

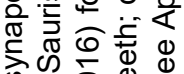
कि ट्ते

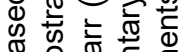
○ 등 운 응 응 它 웡

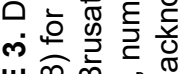
山舟家

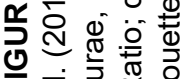



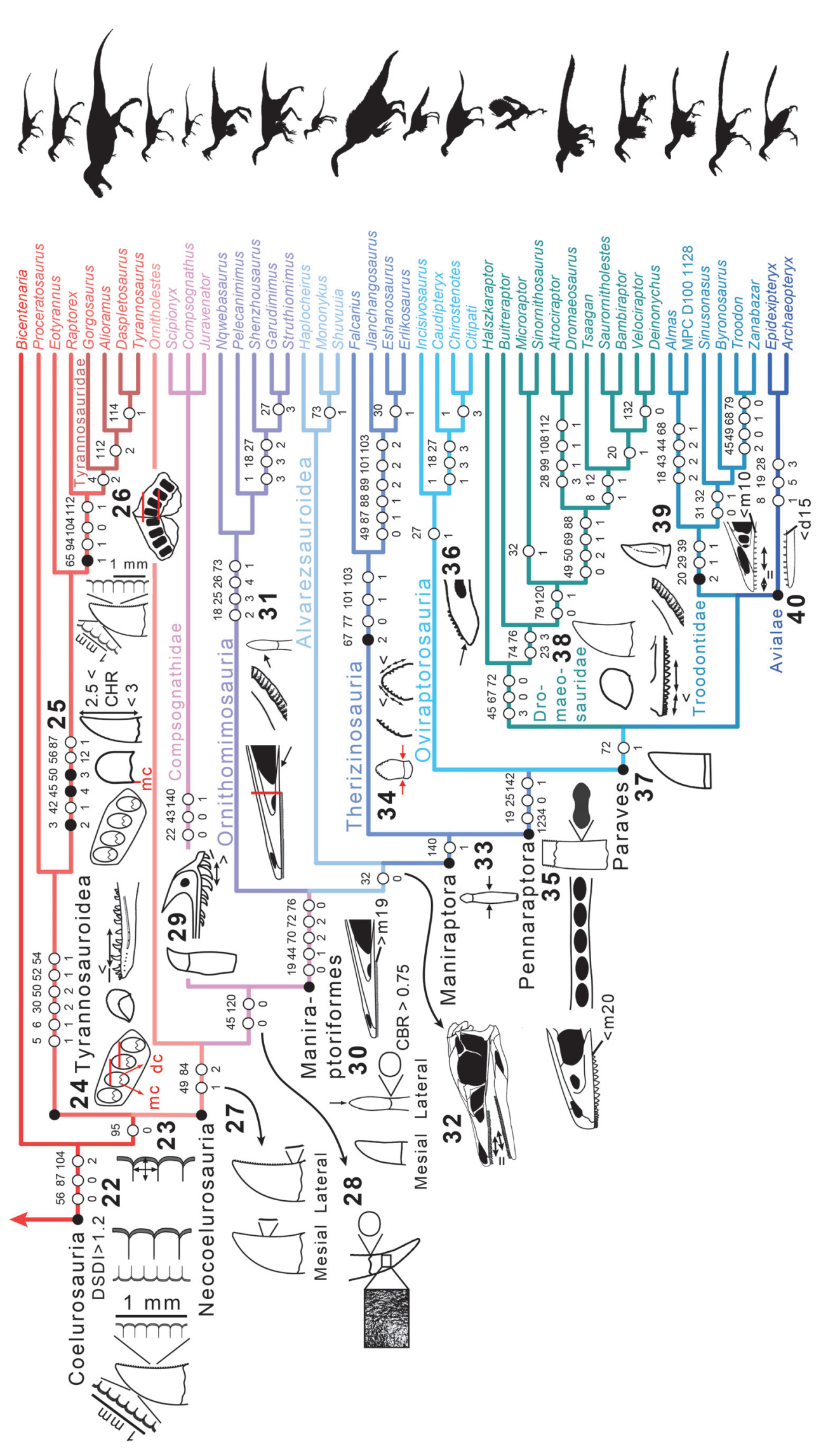

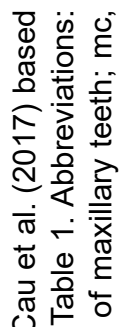

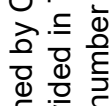

弯 음

Oํㅜㅇ

항 $\frac{0}{0}$

$\frac{\pi}{0} \frac{0}{0}$

둥

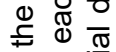

은 흔

을 䗆

흥

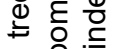

๘ $\frac{10}{\pi} \div$

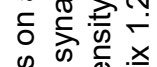

๑) 웜

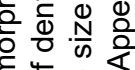

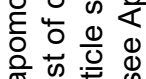

응 武 을

केष 웜

ठ .

制

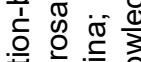

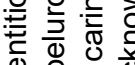

○

.

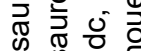

o

ठ원하

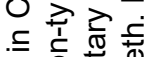

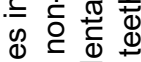

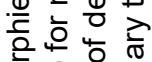

文 $\overline{\overline{0}} \overline{\overline{\bar{x}}}$

을 है

市宁施

के

幽导

त्ञ

노을

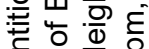

这

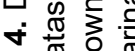

山 중 잉

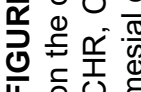


form theropods and Dromaeosauridae. However, fewer than two lateral crown-based autapomorphies characterize most non-avian theropod genera, especially among non-ziphodont theropod clades with simple dentition (i.e., Ornithomimosauria, Alvarezsauroidea, Oviraptorosauria and Troodontidae; Appendices 3.1 and 5, Tree topology 10). Subtle differences in the distribution of dentition-based synapomorphies occur between each non-avian theropod tree represented here. However, most major theropod clades (i.e., Averostra, Avetheropoda, Maniraptoriformes, Neocoelurosauria, Maniraptora and Pennaraptora) are diagnosed by the same number of apomorphic dental features in the large majority of trees (Appendices 3.1 and 5).

With 21 synapomorphies, Spinosauridae is by far the best-supported clade in terms of dentitionbased features (Figure 3; Table 1). It is followed by the clades Spinosaurinae and Allosauroidea, with seven synapomorphies each. With six synapomorphies, the clades Abelisauroidea, Tyrannosauroidea, Pantyrannosauria and Therizinosauroidea are also well-supported by dental characters (Figures 3 and 4; Table 1). On the other hand, no dental synapomorphies support the clades Neotheropoda, Ceratosauria, Tetanurae, Orionides, Alvarezsauroidea and Troodontidae + Avialae (see discussion).

The folidont and putative theropod Chilesaurus shows the most dentition-based, tooth-based and lateral crown-based autapomorphies of any individual theropod genus $(23,16$ and 9, respectively; Appendices 3.1 and 5). It is followed by Epidexipteryx and Sciurumimus (12) as well as Dracovenator and Jianchangosaurus (11), whereas Saurornitholestes and Deinonychus are supported by 10 dentition-based characters. Jianchangosaurus (10), Eoraptor (9), Afrovenator (8), Sciurumimus (8), Acrocanthosaurus (8), Troodon (8) and Dracovenator (7) are also diagnosed by more than six crown-based autapomorphies, whereas Afrovenator (8), Piatnitzkysaurus (6), Eoraptor (6) and Skorpiovenator and Eodromaeus (5 each) display the unique combination of more than four lateral crown-based characters. Out of the 93 toothed taxa included in our data matrix, 74 and 62 are diagnosed by one or more crown-based and lateral crown-based apomorphic characters, respectively. Among the theropods showing apomorphic dental features, 31 are diagnosed by three or more crown-based characters, whereas 38 taxa are diagnosed by two or more lateral crown-based autapomorphies.

\section{Degree of Homoplasy of Dental Features}

Degree of homoplasy and grouping information of dental characters. With values ranging from 0.2075 (tree 4) to 0.209 (tree 5), $\mathrm{Cl}$ is particularly low and relatively similar in all eight trees (Appendix 4.2, Table A1). Likewise, RI values vary slightly between trees and range from 0.448 (tree 4) to 0.4531 (tree 5). With 1210 steps and $\mathrm{Cl}$ and RI of 0.2091 and 0.4531 , respectively, the tree hypothesizing the scansoriopterygid Epidexipteryx as the basalmost oviraptorosaur (tree 5), is the most consistent in terms of dental features (Appendix 4.2, Table A1). On the other hand, the longest tree (tree 4), which provides the least consistent explanation of our dental observations, hypothesizes that megaraptorans are tyrannosauroids (1219 steps; $\mathrm{Cl}$ of 0.2075 and RI of 0.448).

With $\mathrm{Cl}$ and $\mathrm{RI}$ values of 0.689 and 0.707 , respectively, Megalosauroidea $(n=14)$ is the nonavian theropod clade with the least amount of dental homoplasy, and whose dental characters provide the most useful grouping information (Appendix 4.2, Table A2). Ceratosauria $(n=14 ; \mathrm{Cl}$ of 0.683 and RI of 0.653 ) and Allosauroidea ( $n=$ 13 ; Cl of 0.547 ; RI of 0.448 ) show the second and third lowest amount of dental homoplasy, respectively. On the other hand, coelurosaurs $(n=47 ; \mathrm{Cl}$ of 0.341 ; RI of 0.498 ), followed by maniraptoriforms $(n=35$; Cl of 0.378; RI of 0.472) and paravians $(n=19 ; \mathrm{Cl}$ of $0.472 ; \mathrm{Rl}$ of 0.502$)$, have the highest amount of dental homoplasy, whereas dental characters provide the least important grouping information among non-averostran theropods ( $\mathrm{n}=$ 9; $\mathrm{RI}$ of $0.174 ; \mathrm{Cl}$ of 0.513 ) and Dromaeosauridae $(n=11 ; \mathrm{RI}$ of $0.329 ; \mathrm{Cl}$ of 0.51 ).

Excluding dental characters generally improves $\mathrm{Cl}$ and $\mathrm{RI}$ (Figure 5.1). The highest values of $\mathrm{Cl}$ and $\mathrm{RI}$ were obtained when dentitionbased characters are excluded from the dataset in all eight supermatrices, whereas the lowest values of $\mathrm{Cl}$ and $\mathrm{Rl}$ occurred when the dentition-based data matrix was considered separately (Figure 5.1; Appendix 4.2, Table A3). Cl, although strongly correlated with the number of taxa, does not show a significant relationship to the number of characters (Sanderson and Donoghue, 1989). Cl values show little variation when dental characters are either included or excluded for the supermatrices of Choiniere et al. (2014a) and Lee et al. (2014a) (Figure 5.1; Appendix 4.2, Table A3). Mann-Whitney U-test scores based on $\mathrm{Cl}$ values confirm that the difference between dental and non-dental characters is not statistically significant for those two datasets ( $p$-values > 0.05; Table 2; Appendix 4.2, Table A4). 
TABLE 1. List of unambiguous dental synapomorphies in 40 theropod clades illustrated in Figures 3 and 4 and based on a data matrix of 145 dental characters coded in 97 saurischian taxa.

\begin{tabular}{ll}
\hline \multicolumn{1}{c}{ Clade } & \multicolumn{1}{c}{ Unambiguous dental synapomorphy } \\
\hline $\begin{array}{l}\text { 1. Eoraptor + Theropoda } \\
\text { 2. Dracovenator + Neotheropoda }\end{array}$ & $\begin{array}{l}\text { Character 4: partial overlap of the first and second premaxillary alveoli in palatal view } \\
\text { Characters 15, 120: slightly constricted premaxillary tooth row in palatal view; braided } \\
\text { oriented enamel surface texture not clearly visible with light }\end{array}$ \\
$\begin{array}{l}\text { 3. Liliensternus + Dilophosaurus + } \\
\text { Averostra }\end{array}$ & $\begin{array}{l}\text { Character 31: first dentary tooth/alveolus substantially smaller than second and third } \\
\text { dentary teeth/alveoli }\end{array}$ \\
4. Dilophosaurus + Averostra & $\begin{array}{l}\text { Characters 56: } 14 \text { to } 19 \text { mid-crown denticles per five millimeters on the distal carina (DC) } \\
\text { of mesial teeth in subadults/adults }\end{array}$ \\
5. Averostra & $\begin{array}{l}\text { Character 15: unconstricted premaxillary tooth row in palatal view } \\
\text { 6. Abelisauroidea }\end{array}$ \\
& $\begin{array}{l}\text { Characters 8, 9, 45, 46, 51: mesial and distal premaxillary teeth subequal in size than the } \\
\text { first six mesial maxillary teeth (or alveoli); salinon-shaped outline of basal cross-section of } \\
\text { the crown in the mesialmost tooth; concave surfaces adjacent to the mesial distal carina } \\
\text { on the lingual side of the mesial teeth; mesial carina extends to the cervix or just above it } \\
\text { in mesial teeth }\end{array}$
\end{tabular}

7. Ceratosauridae

Characters 5, 69, 94: overlap of the second and third premaxillary alveoli in palatal view; largest crown in subadults/adults higher than six centimeters; subquadrangular mesial denticles at two-thirds of the crown in lateral teeth

8. Noasauridae

Characters 31, 40: first dentary tooth/alveolus subequal size in than second and third dentary alveoli; largest crown in subadults/adults shorter than one centimeter

9. Abelisauridae

Characters 3, 4, 13, 25, 91, 99: premaxillary alveoli all mesio-distally oriented; no overlap of the first and second premaxillary alveoli in palatal view; subrectangular premaxillary and maxillary alveoli; distal denticles hooked and apically inclined from distal margin in lateral teeth

10. Megalosauroidea Character 82: mesial carina extending to base of crown or slightly above the cervix in lateral teeth

11. Piatnitzkysauridae

Characters $19,104,108,112,113: 18$ to 19 maxillary teeth/ alveoli; distal denticles larger than mesial ones in lateral crowns (DSDI > 1.2); short and poorly developed interdenticular sulci between mid-crown denticles on the distal carina in lateral teeth; tenuous and numerous transverse undulations on the crown surface of lateral teeth

12. Megalosauria

Character 53: mesial carina facing labially in mesialmost teeth

13. Megalosauridae

Character 28: fewer than 15 dentary teeth/alveoli

14. Megalosauridae more derived than Sciurumimus

15. Spinosauridae

Characters $5,56,108,109$ : overlap of the second and third premaxillary alveoli in palatal view; nine to 13 denticles per five millimeters on the distal carina at mid-crown in mesial teeth; short and poorly developed interdenticular sulci between basal and mid-crown denticles on the distal carina in lateral teeth

Characters 2, 7, 8, 11, 12, 14, 15, 22, 23, 25, 30, 33, 45, 51, 63, 70, 76, 82, 110, 141, 142: more than six premaxillary teeth; distal premaxillary teeth smaller than mesial premaxillary teeth; mesial premaxillary and maxillary teeth subequal in size; second premaxillary tooth/ alveolus significantly smaller than third and fourth tooth/alveolus; distalmost premaxillary tooth significantly smaller than more mesial teeth; premaxillary teeth anterior to external naris; strongly lateromedially constricted premaxillary tooth rows; first maxillary tooth/ alveolus significantly smaller than second tooth/alveolus; procumbent mesial maxillary teeth; subcircular maxillary alveoli; mesialmost dentary teeth significantly larger than more distal ones; terminal rosette of the dentary bearing four teeth; outline of the basal crosssection of mesial teeth subcircular; mesial carina extending well-beneath the cervix in mesial teeth and to the base of crown or slightly above the cervix in lateral teeth; flutes present on the lingual surface of mesial teeth; distal margin of lateral teeth convex, with apex centrally positioned; $C B R>0.75$ in lateral teeth; subcircular cross-sectional outline of lateral teeth; flutes on both lingual and labial sides of lateral teeth; root strongly tapered apically and oval to subcircular in cross-section at mid-root

16. Avetheropoda

Characters 41, 50, 51: labiolingual compression of the crown weak $(0.75<\mathrm{CBR}<1.2$, tooth subcircular) in mesial teeth; mesial carina of mesial teeth slightly twisted, curves onto the mesiolingual surface; mesial carina extends to the cervix or slightly above it in mesial teeth 
TABLE 1 (continued).

\section{Clade}

17. Allosauroidea

18. Metriacanthosauridae

19. Allosauria

20. Neovenatoridae

21. Carcharodontosauridae

22. Coelurosauria

23. Tyrannosauroidea + Neocoelurosauria

24. Tyrannosauroidea

25. Pantyrannosauria

26. Tyrannosauridae

27. Neocoelurosauria

28. Compsognathidae + Maniraptoriformes

29. Compsognathidae

30. Maniraptoriformes

31. Ornithomimosauria

32. Alvarezsauroidea + Maniraptora

33. Maniraptora
Unambiguous dental synapomorphy

Characters $5,46,55,56,87,94,108,112$ : overlap of the second and third premaxillary alveoli in palatal view; concave surface on the labial and lingual surfaces adjacent to the distal and mesial and distal carinae, respectively, in mesial teeth; nine to 13 mesial and distal denticles per five millimeters in mesial teeth, and nine to 15 in the mesial carina of lateral teeth; subquadrangular mesial denticles at two-thirds height of crown in lateral teeth; short and poorly developed interdenticular sulci between distal denticles at midcrown in lateral teeth; tenuous or well-visible transverse undulations on the crown surface of lateral teeth

Characters 74,120 : surface centrally positioned on the labial surface of the crown roughly flattened in lateral teeth; irregular and non-oriented enamel surface texture

Characters 9, 109: distal premaxillary teeth/alveoli subequal in size than the first six mesial maxillary teeth/alveoli; short and poorly developed interdenticular sulci in basal denticles of the distal carina

Characters $22,74,75$ : first maxillary tooth significantly smaller than second maxillary tooth; surface centrally positioned on the labial surface of the crown roughly flattened in lateral teeth; concave surface adjacent to the distal carina on the labial and lingual surfaces, in lateral teeth

Characters 45, 46, 97: subcircular, ovoid or elliptical outline of basal cross-section of the crown in the mesialmost tooth; concave surface adjacent to the carina absent in mesial teeth; biconvex mesial denticles

Characters 56, 87, 104: more than 20 distal denticles at mid-crown and more than 30 mesial denticles at two-thirds height of the crown in mesial and lateral teeth, respectively; distal denticles larger than mesial ones in lateral crowns (DSDI >1.2)

Character 95: mid-crown denticles (DC) on distal carina as long mediodistally as apicobasally, subquadrangular, in lateral teeth

Characters $5,6,30,50,52,54$ : overlap of the second and third as well as third and fourth premaxillary alveoli in palatal view; mesialmost dentary teeth significantly smaller than mid- and distal dentary teeth; mesial carina of mesial teeth strongly twisted, curving onto the lingual surface; distal carina of mesial teeth strongly labially deflected and facing mostly lingually

Characters $3,42,45,50,56,87$ : all premaxillary alveoli labio-lingually oriented; important baso-apical elongation of the crown $(2.5<\mathrm{CHR} \leq 3)$ in mesial teeth; U-shaped outline of basal cross-section of the crown in the mesialmost tooth; mesial carina almost straight and strongly lingually deflected in mesial teeth; fewer than 20 distal denticles per five millimeters at mid-crown in the mesial dentition of subadults/adults, and fewer than 30 mesial denticles per five millimeters at two-thirds height in the lateral dentition

Character 4: overlap of the first and second premaxillary alveoli in palatal view almost complete

Characters 49, 84: unserrated distal carina in mesial teeth; distal carina extends wellabove the cervix in lateral teeth

Characters 45, 120: subcircular outline of basal cross-section of the crown in the mesialmost tooth; smooth or irregular non-oriented enamel surface texture of the crown

Characters 22, 43, 140: First maxillary tooth significantly smaller than second maxillary tooth; strongly distally recurved mesial teeth; root with convex mesial and distal margins, root significantly larger than base crown

Characters $19,44,70,72,76$ : more than 19 maxillary teeth; distal margin of the mesial crowns straight in lateral view; weak (i.e., CBR > 0.75) labiodistal compression of the lateral crowns; margin of crown convex, so that the crown apex of lateral teeth is centrally positioned; subcircular cross-sectional outline at the base crown in lateral teeth

Characters 18, 25, 26, 73: maxillary teeth absent in the anteriormost and/or most of the posterior portion of the maxilla; maxillary alveoli merged to form an open groove; distalmost maxillary tooth aligned to the anteroventral rim of the antorbital fenestra; mesial margin of lateral teeth slightly convex, almost straight

Character 32: mid-dentary teeth/alveoli subequal in size than mesial maxillary teeth/alveoli

Character 140: root with convex margins, root significantly larger than crown base 
TABLE 1 (continued).

\begin{tabular}{|c|c|}
\hline Clade & Unambiguous dental synapomorphy \\
\hline 34. Therizinosauria & $\begin{array}{l}\text { Characters } 67,77,101,103 \text { : important constriction at the cervix in lateral teeth, base of } \\
\text { crown occupying } 85 \% \text { or less of largest crown width; mesial carina present in lateral teeth; } \\
\text { lower number of denticles apically than at the mid-crown on the mesial and distal carinae } \\
\text { in lateral teeth }\end{array}$ \\
\hline 35. Pennaraptora & $\begin{array}{l}\text { Characters } 19,25,142 \text { : fewer than } 19 \text { maxillary teeth/alveoli; oval to lenticular maxillary } \\
\text { alveoli; } 8 \text {-shaped outline of mid-root in cross-section }\end{array}$ \\
\hline 36. Oviraptorosauria & Character 27: teeth absent in the anteriormost portion of the dentary \\
\hline 37. Paraves & $\begin{array}{l}\text { Character } 72 \text { : distal margin of lateral crown slightly concave, roughly straight, or straight, } \\
\text { apex positioned at the same level as distal profile }\end{array}$ \\
\hline 38. Dromaeosauridae & $\begin{array}{l}\text { Characters } 45,67,72 \text { : D-shaped or J-shaped cross-sectional outline of the base crown in } \\
\text { mesial teeth; constriction between crown and root absent in lateral teeth; distal margin of } \\
\text { lateral crown strongly concave }\end{array}$ \\
\hline 39. Troodontidae & $\begin{array}{l}\text { Characters } 20,29,39 \text { : mesial maxillary teeth significantly smaller than distal maxillary } \\
\text { teeth; dentary alveoli merged to form an open groove; constriction between crown and } \\
\text { root present in all lateral teeth }\end{array}$ \\
\hline 40. Avialae & $\begin{array}{l}\text { Characters } 8,19,28 \text { : mesial premaxillary teeth subequal in size than the first six mesial } \\
\text { maxillary teeth; fewer than ten maxillary teeth; fewer than } 15 \text { dentary teeth }\end{array}$ \\
\hline
\end{tabular}

$\mathrm{Cl}$ values between dental and non-dental characters are significantly different (t-test and MannWhitney U-test with $\mathrm{p}$-values $<0.05$ ) for all other supermatrices (Figure 5.1). There are also significant differences in RI for dental and non-dental characters for all eight supermatrices (Table 2). Cl values are lower for dental characters in all eight supermatrices and range from 0.239 to 0.591 in all datasets. The highest $\mathrm{Cl}$ values for dental characters are found in two datasets on ceratosaurs [i.e., Rauhut and Carrano (2016) and Tortosa et al. (2014); Appendix 4.2, Table A4]. Similarly, dental characters always show lower RI values, varying from 0.379 to 0.559 , with the highest value obtained for the dataset of Tortosa et al. (2014; ceratosaurs; Appendix 4.2, Table A4).

Degree of homoplasy and grouping information of each dental sub-units/characters. With values of 0.62 and 0.565 , the dentition sub-units showing the highest $\mathrm{Cl}$ scores are the crown enamel texture and microstructure ( $n$, the total number of characters on crown enamel texture and microstructure in the dataset $=20$ ) as well as the mesial crown ornamentations ( $n=4)$, respectively (Figure 5.2; Appendix 4.2, Table A5). The lowest $\mathrm{Cl}$ are obtained for the carina $(0.183 ; n=8)$ and crown $(0.215 ; n=9)$ morphology in the mesial dentition, but also for the carina $(0.216 ; n=10)$, denticle $(0.222 ; n=23)$ and crown $(0.216 ; n=10)$ morphology of the lateral dentition (Figure 5.2). Characters on the premaxillary $(n=17)$, maxillary $(n=9)$ and dentary teeth $(n=10)$ as well as on the root mor- phology $(n=6)$ also show particularly low $\mathrm{Cl}$ values (average values from 0.229 to 0.286 ). The highest $\mathrm{RI}$ scores are for the enamel texture and microstructure (0.58), premaxillary teeth (0.472) and carina morphology of both mesial and lateral dentitions ( 0.46; Figure 5.2). The lowest RI scores are for the lateral crown ornamentations $(0.231 ; n=10$; Figure 5.2). Most of the 34 dental features that we highlight have $\mathrm{Cl}$ scores below 0.3 and $\mathrm{RI}$ scores above 0.4 . This is particularly the case for lateral dentition characters, half of which have RI values higher than 0.45 (Appendix 4.2, Table A6). ANOVAs show that there is no statistically significant difference of variance in RI values between each of the dentition sub-units (Appendix 4.2, Table A8). On the other hand, the difference of variance in $\mathrm{Cl}$ between enamel characters and characters on the premaxillary and dentary teeth, mesial and lateral crown morphology, carina morphology, and lateral denticle morphology is statistically significant (Appendix 4.2, Table A7).

\section{DISCUSSION}

\section{Summary}

- The high levels of homoplasy exhibited by dental features result from a large amount of convergence in the dentition of distantly related theropod taxa with similar feeding strategies.

- Dental characters are the least homoplastic and provide the most important grouping 


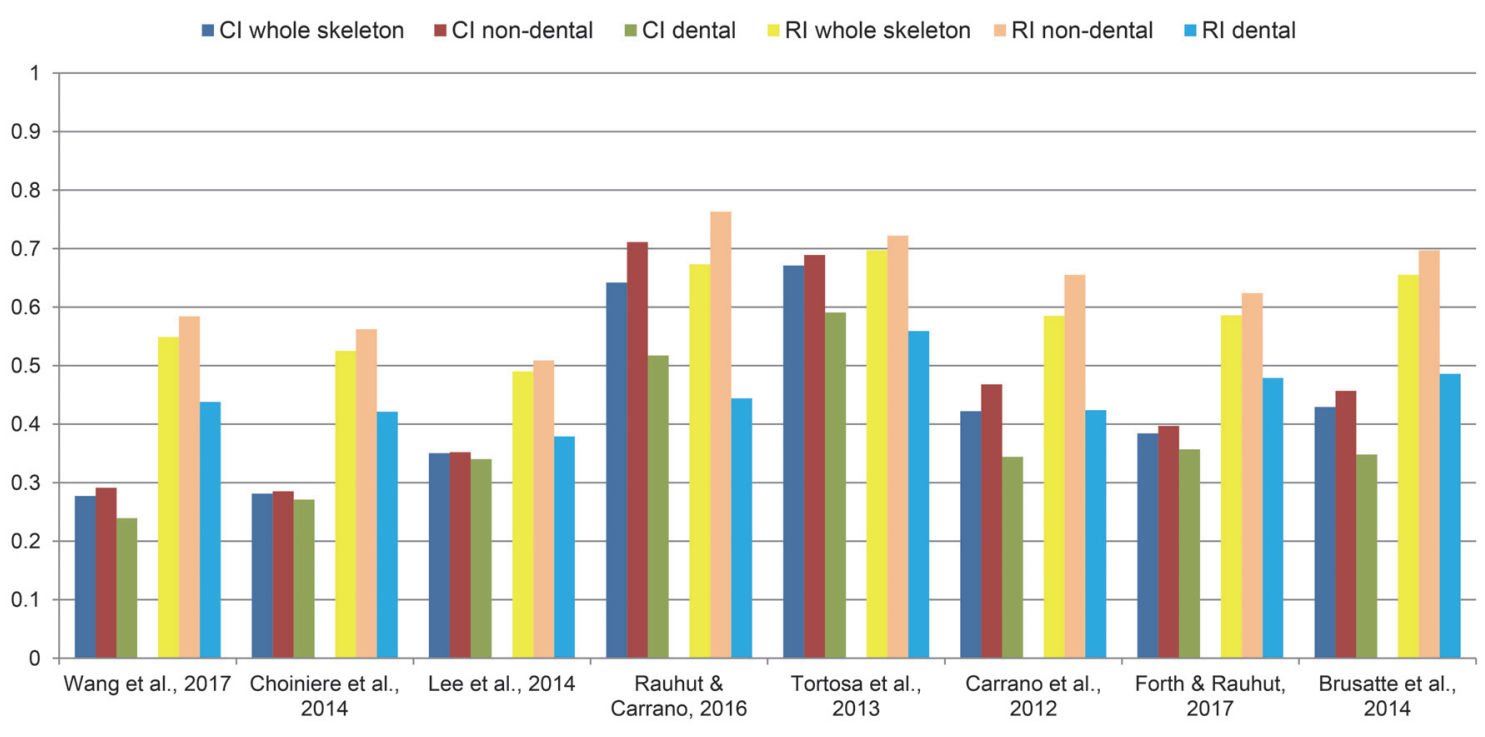

ai $\square$ ri

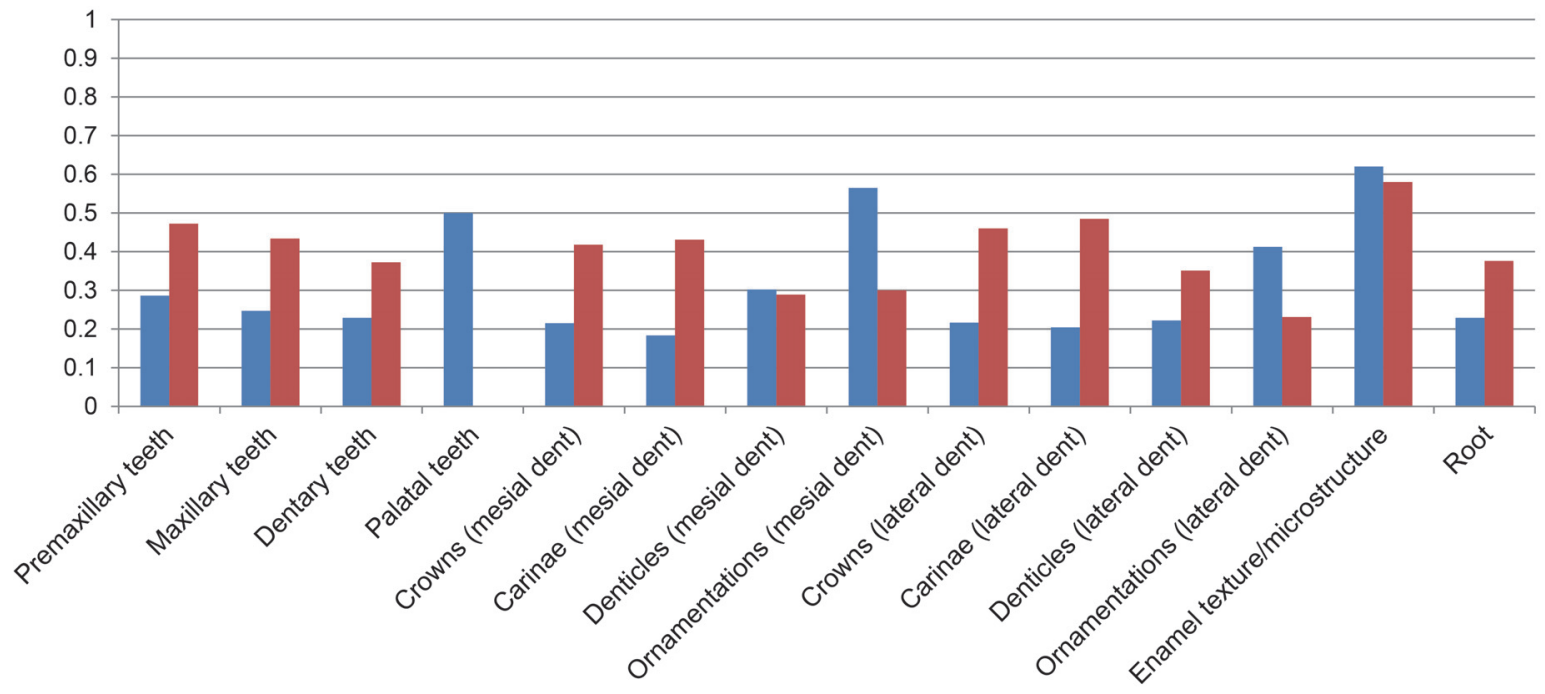

FIGURE 5. Cl and RI scores obtained in eight supermatrices separated into skeleton-based, non-dental based and dental-based data matrices, and $\mathrm{Cl}$ and $\mathrm{RI}$ values for each dentition sub-unit. 1, Cl and RI scores in eight supermatrices combining our dentition-based data matrix to eight of the most recent datasets on non-avian theropods, ceratosaurs, non-coelurosaur tetanurans and non-avian coelurosaurs, when dental characters are included (whole skeleton), excluded (non-dental), and considered separately (dental); 2, Cl and RI values measured for each dentition sub-unit in eight trees of the theropod classification (see Appendix 4.2, Table A5). Abbreviations: dent, dentition.

information for Megalosauroidea and Ceratosauria. This results from the highly specialized dentition of Spinosauridae and the diagnostic dentition of Ceratosauridae and Abelisauridae.

- Dental features always convey less grouping information than characters derived from the rest of the skeleton. Nevertheless, over broad phylogenetic ranges such as nonavian theropods, dentition-related characters might be just as homoplastic as nondental characters.

- Crown ornamentations and microstructure should be prioritized when attempting to ascribe theropod teeth to a certain taxon as they are the least homoplastic dental sub- 
unit and convey the most important grouping information.

- Among the 34 dental features we highlight, longitudinal ridges and flutes on the crown are the least homoplastic. Additionally, crown height and thickness, the presence and extension of the mesial carina, the cross-sectional outline and enamel surface texture convey the most important grouping information. Conversely, the absence of denticles on the mesial carina, a labially deflected distal carina, the shape of mesial/ distal denticles, the difference in size between mesial and distal denticles, and the presence and development of transverse/ marginal undulations and interdenticular sulci provide little grouping information.

- Few theropod taxa bear diagnostic crowns that can be identified at the genus level due to morphological convergence and the variability of dental features (e.g., extension of the mesial carina, presence of mesial denticles, labial/lingual depressions, longitudinal ridges, marginal and transverse undulations) along the tooth row in a single individual.

- Most isolated theropod shed teeth, which are typically from the lateral dentition, do not show taxonomic precision sufficient for finescale biostratigraphic analysis. Nevertheless, some theropod taxa such as Majungasaurus, Piatnitzkysaurus, Afrovenator, Acrocanthosaurus, Tyrannosaurus, Saurornitholestes, and Troodon have highly diagnostic teeth that may be useful biostratigraphic markers.

- The most important evolutionary transition in the dentition of theropod dinosaurs occurred with the emergence of Spinosauridae, which are characterized by a highly specialized dentition showing a strong adaptation towards piscivory. Several authors have proposed a list of evolutionary steps leading to the derived dentition of Spinosauridae based on putative spinosaurid teeth from the Jurassic of Africa. These isolated teeth, however, likely belong to non-spinosaurid theropods so that the timing and novelty sequence of the apomorphic dental characters displayed by spinosaurids (e.g., flutes, minutes denticles, veined enamel texture) remain unknown.

- Two important evolutionary steps in the dentition of theropods occurred during the radiation of Allosauroidea and Tyrannosauroidea.
Both clades evolved independently transversely thick asymmetrical mesial teeth with J-shaped and/or salinon-shaped cross-sectional outline and a mesial carina that migrated mesiolingually. These evolutionary changes can be functionally explained by an anteroposterior shortening of the premaxilla possibly as the result of adaptation to a diet involving increased levels of bone-crunching and bone-biting, with a high degree of torsion applied on the mesial dentition.

- The most important transition in the evolution of the coelurosaur dentition, marked by tooth simplification, occurred with the radiation of Maniraptoriformes and likely results from a trophic shift between carnivory and herbivory. The changes in tooth morphology can be summarized by three evolutionary steps: i) a loss of mesial denticles in both mesial and lateral teeth, and a loss of distal denticles in mesial teeth in basal neocoelurosaurs; ii) the development of conical mesial teeth and an irregular enamel surface texture in the clade Compsognathidae + Maniraptoriformes; and iii) an increase in the number of maxillary teeth, the loss of a distal curvature in both mesial and lateral teeth, and the development of lateral teeth with a subcircular outline in Maniraptoriformes.

\section{Taxonomic Potential of Theropod Teeth}

Homoplasy and grouping information in theropod dental features. Dentition-based characters exhibit high levels of homoplasy among non-avian theropods $(\mathrm{Cl}$ values are $\sim 0.21$ for each of the theropod trees we examined). However, the homoplasy present in dental characters is apportioned in such a way that they still provide useful grouping information and are thus potentially of taxonomic value ( $\mathrm{RI}$ values $\sim 0.45$ ). $\mathrm{Cl}$ and $\mathrm{RI}$ increase when dental characters are not taken into consideration in all eight supermatrices (Figure 5.1), indicating that non-dental characters are typically less homoplastic and convey more grouping information than dentition-based features. Megalosauroids and ceratosaurs were revealed to be the theropod clades with the least amount of dental homoplasy. Dental characters also provide the most important grouping information in these two clades (Appendix 4.2, Table A2). High $\mathrm{Cl}$ and RI values in Megalosauroidea and Ceratosauria likely result from the derived and highly peculiar dentition of Spinosauridae (see below) and the diagnostic dentition of Ceratosauridae and Abelisauridae. $\mathrm{Cl}$ and $\mathrm{RI}$ val- 
TABLE 2. $\mathrm{p}$-values of the t-test and Mann-Whitney U-test (M-W) for $\mathrm{Cl}$ and $\mathrm{RI}$ values between dental and no-dental characters in five datasets of the theropod skeleton. Significantly similar values between dental and non-dental sets of characters are bolded.

\begin{tabular}{lccccc}
\hline \multicolumn{1}{c}{ Dataset } & Clade & t-test, ci & t-test, ri & M-W, ci & M-W, ri \\
\hline Brusatte et al. (2014) & Non-avian Coelurosauria & $2.14 \mathrm{E}-10$ & $4.67 \mathrm{E}-14$ & $6.77 \mathrm{E}-10$ & $2.26 \mathrm{E}-15$ \\
Carrano et al. (2012) & Non-coelurosaur Tetanurae & $2.29 \mathrm{E}-09$ & $5.42 \mathrm{E}-13$ & $4.61 \mathrm{E}-10$ & $1.03 \mathrm{E}-12$ \\
Choiniere et al. (2014a) & Non-avian Theropoda & $\mathbf{0 . 2 2 0 2 5}$ & $7.31 \mathrm{E}-07$ & $\mathbf{0 . 4 4 8 3}$ & $2.01 \mathrm{E}-07$ \\
Foth and Rauhut (2017) & Non-avian Coelurosauria & 0.001355 & $1.66 \mathrm{E}-07$ & 0.006778 & $1.69 \mathrm{E}-07$ \\
Lee et al. (2014a) & Non-avian Theropoda & 0.014723 & $2.43 \mathrm{E}-08$ & $\mathbf{0 . 0 9 6 9 0 4}$ & $2.71 \mathrm{E}-08$ \\
Rauhut and Carrano (2016) & Ceratosauria & $5.47 \mathrm{E}-09$ & $1.99 \mathrm{E}-10$ & $4.15 \mathrm{E}-08$ & $5.93 \mathrm{E}-10$ \\
Tortosa et al. (2014) & Ceratosauria & 0.015288 & 0.0088158 & 0.018854 & 0.0077152 \\
Wang et al. (2017a) & Non-avian Theropoda & 0.0027452 & $9.99 \mathrm{E}-08$ & 0.0005687 & $2.19 \mathrm{E}-08$ \\
\hline
\end{tabular}

ues obtained in each non-avian theropod clade also reveal that the amount of dental homoplasy and the usefulness of dental characters in providing grouping information are neither greater nor lower in basal or derived theropod lineages.

The data matrices of Choiniere et al. (2014a) and Lee et al. (2014a), which returned relatively similar $\mathrm{Cl}$ values regardless of dental character inclusion/exclusion from the supermatrices (Figure 5.1; Table 2; Appendix 4.2, Table A4), are two out of three of the larger, more comprehensive datasets in non-avian theropods, and thus large differences in homoplasy levels between dental and non-dental characters in other smaller datasets might be an artifact of low character and taxon sample size in other theropod datasets. These results may also indicate that over broad phylogenetic ranges, or when huge numbers of characters are sampled, dental characters might be just as homoplastic as other characters. Nevertheless, significant differences of $\mathrm{Cl}$ values between dental and non-dental characters in Wang et al.'s (2017a) dataset on non-avian theropods, which is the second largest used in this study, suggests that characters on the dentition tend to be more homoplastic than the rest of the skeleton even over broad phylogenetic ranges. The significant differences in $\mathrm{RI}$ among dental and non-dental characters in all eight supermatrices (Table 2), and when dental characters are included and excluded from each of these datasets, clearly indicate that dental features always convey less grouping information than the rest of the skeleton. The highest $\mathrm{Cl}$ values for dental characters were obtained from the datasets of Tortosa et al. (2014) and Rauhut and Carrano (2016), which show 0.5 to 0.6 values, respectively. This suggests that dental features are less homoplastic for ceratosaurs. This may result from the low sample size of these datasets, which only con- tain 15 to 16 taxa. Analysis of clades within datasets with more characters shows that ceratosaurs, as well as megalosauroids, have less dental homoplasy than other theropod lineages. Meaningful differences, which cannot be attributed to known effects of sample size on $\mathrm{Cl}$ and $\mathrm{RI}$, indeed appear to be present between groups of relatively similar sample sizes that have independent lineage histories such as Ceratosauria, Megalosauroidea, Allosauroidea, and Dromaeosauridae. RI values for dental characters range from 0.42 to 0.48 in the datasets dealing with ceratosaurs, non-coelurosaur tetanurans and non-avian coelurosaurs, providing relatively similar grouping information in each of these clades. Dental characters provide more useful grouping information in Megalosauroidea and Ceratosauria than in Allosauroidea, Coelurosauria, Maniraptoriformes or Paravians.

The high degree of homoplasy among dental features can be explained by a large amount of convergence in the dentition of distantly related theropod taxa with similar feeding strategies. The lateral dentitions of ceratosaurids, piatnitzkysaurids, allosauroids, basal tyrannosauroids and dromaeosaurids have many dental features in common and only subtle differences such as the extension of the mesial carina on the crown, the difference in size between mesial and distal denticles and the presence and extension of labial and/ or lingual depressions on the crown can differentiate them (C.H. personal obs.). This also explains the strong homoplasy displayed by characters on premaxillary, maxillary and dentary teeth, as well as the crown, carina and denticle morphology of both mesial and lateral dentitions. All these dental features appear to be dominated by functional constraints so that distantly related theropods with similar feeding strategies will rapidly and convergently acquire these characters throughout their evolu- 
tion. On the other hand, crown ornamentations $(\mathrm{Cl}$ of 0.49 ) and microstructure $(\mathrm{Cl}$ of 0.62$)$, although bearing functional properties and linked to diet (e.g., Sander, 1999; Brink et al., 2015, 2016; Wang et al., 2015), are the least homoplastic possibly because they require more complex developmental/genetic mechanisms to evolve than other dental features under the same evolutionary pressure. Indeed, crown microstructure has been suggested to bear some phylogenetic potential in dinosaurs (Hwang, 2005, 2010; Wang et al., 2015).

The ANOVA test found statistically significant differences in $\mathrm{Cl}$ values between characters of the enamel morphology and premaxillary teeth, mesial and distal crown, carina morphology, and lateral denticle morphology (Appendix 4.2). This is due to the particularly high values of $\mathrm{Cl}$ in enamel-related characters, with seven out of 17 characters on the enamel microstructure having $\mathrm{Cl}$ and $\mathrm{RI}$ scores of 1. Nevertheless, such high $\mathrm{Cl}$ and RI values for characters on enamel microstructure should be considered cautiously as they obviously result from low sampling size. Information on crown histology was taken from Hwang (2007), who investigated the enamel microstructure in 24 distantly related theropod taxa, of which 15 are included in our data matrix. In addition, eight of the characters provided by Hwang (2007) are applicable only to 10 taxa, and five of the characters are restricted to six taxa. Interestingly, Hwang $(2005,2007)$ observed a large amount of homoplasy in enamel microstructure among theropods but the results of our study appear to show the reverse pattern. Our study suggests that, among all dental characters displayed by isolated shed teeth, features on enamel texture and microstructure convey the most important grouping information ( $\mathrm{RI}$ is 0.58 ) of all dental subunits and should be investigated first in order to assign theropod teeth to taxa with more confidence. Our low sampling size might nonetheless negate the effects of Hwang's (2007) larger dataset as increasing numbers of taxa drives these metrics down in a predictable pattern.

Homoplasy and grouping information in the $\mathbf{3 4}$ dental characters highlighted. Low $\mathrm{Cl}$ scores in most of the 34 dental characters examined $(\mathrm{Cl}$ values of 0.251 ) demonstrate that most of these features are strongly homoplastic $(\mathrm{Cl}$ is less than 0.3 , and typically around 0.2; Appendix 4.2, Table A6). Longitudinal ridges $(\mathrm{Cl}$ is 0.75$)$ and flutes $(0.54)$ on the crown are the dental characters with the least amount of homoplasy and the only characters with $\mathrm{Cl}$ values higher than 0.5 . Nonetheless, significantly higher RI values (RI is 0.39 ) reveal that many dental characters provide some grouping information and can still be optimized as local synapomorphies of less inclusive theropod clades. This is particularly the case of dental features on the lateral dentition and characters related to: crown thickness $(R I=0.59)$, height $(R I=0.54)$, presence of the mesial carina $(R I=0.57)$, extension of the mesial carina $(\mathrm{RI}=0.52)$, cross-sectional outline $(R I=0.6)$, and enamel surface texture $(\mathrm{RI}=0.61)$.

With $\mathrm{Cl}$ and $\mathrm{RI}$ equal to 1 , when characters on enamel microstructure are excluded, subrectangular alveoli in the premaxilla (found in Abelisauridae only) and a mesial dentition bearing a longitudinal ridge centrally positioned on the lingual surface [seen in Tyrannosauridae and Raptorex, a possible juvenile of Tarbosaurus (Fowler et al., 2011b)] are the only non-homoplastic and uniquely synapomorphic dental characters of theropod subclades (Appendix 4.2, Table A5). With RI higher than 0.7, a premaxillary tooth row anterior to the external naris, the shape of the maxillary alveoli, the spacing of the dentary teeth, the presence of serrations on the distal carina and a twisted mesial carina in mesial teeth, the presence of flutes in lateral teeth, and the presence of minute or very large denticles along the distal carina in the mesial and lateral teeth, are dental characters that also convey important grouping information and have potential taxonomic value. Likewise, with RI higher than 0.6, the presence of an alveolar groove in the dentary, the crown thickness and cross-sectional outline, and the extension of the mesial carina, as well as the presence of a constriction between crown and root, the extension of the mesial and distal carinae and the shape of mesial denticles at two-third of the crown height in the lateral dentition, are dental features that provide useful grouping information.

Among the 34 dental features we highlight, additional features that convey relatively good grouping information are: basal constriction at the cervix in lateral teeth (RI of 0.625), absence of mesial and distal carinae ( 0.59 and 0.5$)$, cross-sectional outline in lateral teeth (0.57), and straight or convex distal profile in the lateral crown (0.58). On the other hand, with an RI score equal or lower than 0.4 , procumbent premaxillary, maxillary or dentary teeth ( $R \mathrm{I}$ is 0.14 on average), absence of denticles on the mesial carina (0.29), a labially deflected distal carina (0.23), the shape of mesial and distal denticles (0.24), distal denticles significantly smaller/larger than mesial denticles (0.29), transverse and marginal undulations (0.31), and the presence and development of interdenticular 
sulci between mid-crown distal denticles (0.25) provide little grouping information. Finally, with RI equal to 0 , procumbent premaxillary teeth, the presence of basal striations, denticles on the mesial carina and interdenticular sulci between distal denticles in mesial teeth, the shape of mesial denticles and the presence of longitudinal ridges in lateral teeth, as well as the presence of longitudinal grooves in mesial and lateral teeth are dental features that are optimizing as localized autapomorphies of phylogenetically distant taxa and, consequently, do not convey grouping information. The combination of these features may, nonetheless, narrow down the phylogenetic distribution of isolated teeth to a certain taxon.

Distribution of dental characters in theropods and their biostratigraphic value. The distribution of dentition-based and crown-based characters on theropod trees reveals that dental features tend to better-diagnose 'super-family' and 'family' level clades than major theropod clades such as Ceratosauria, Maniraptora, Orionides, Paraves and Tetanurae (Appendix 5). The distribution of crownbased features has also shown that most theropod OTUs are diagnosed by fewer than three crownbased autapomorphies and fewer than two lateral crown-based apomorphic characters (Appendix 5, Tree topology 9 and 10). This suggests that few theropod taxa bear diagnostic crowns that can be identified at the genus level, and that most isolated theropod shed teeth, which are typically from the lateral dentition, do not show taxonomic precision sufficient for fine-scale biostratigraphic analysis. Taxa diagnosed by four lateral crown-based autapomorphies or more include Acrocanthosaurus, Afrovenator, Chilesaurus, Majungasaurus, Megaraptor, Piatnitzkysaurus, Saurornitholestes, Sciurumimus, Skorpiovenator, Troodon and Tyrannosaurus. Several authors such as Baszio (1997), Fiorillo and Gangloff (2001), Smith et al. (2005), and Fanti and Therrien (2007) have successfully identified isolated crowns to these taxa, and biostratigraphic correlations of the deposits containing these fossils can be inferred based on theropod shed teeth only (Larson and Currie, 2013).

Many theropod shed teeth are, however, not diagnostic to the genus level due to both morphological convergence and to the variability of dental features along the tooth row within a single individual. For example, the crowns of Megalosauridae, Carcharodontosauridae and Dromaeosauridae are typically characterized by the presence of a mesial carina not reaching the cervix (Hendrickx et al., 2015c), pronounced marginal undulations (Brusatte et al., 2007), and longitudinal ridges and/or deep, apicobasally extended labial/lingual depressions (Gianechini et al., 2011b; Evans et al., 2013), respectively. But each of these features is varyingly represented along the tooth row (Figure 6). The dentition of Acrocanthosaurus atokensis (NCSM 14345), for instance, displays strong marginal undulations in $R m \times 3$ whereas $R m \times 1, R m \times 2$ and Rmx4 lack these undulations (Figure 6.1). The same appears for transverse undulations that are well-visible on the labial surface of $\mathrm{Rmx} 1$ to 6 but only seen on the lingual surface in Lmx2 and Lmx4 (personal obs.). In the left maxilla of Alioramus altai (MPC-D 100-1844), a lingual depression is visible in some teeth but absent in others, while the mesial carina reaches the cervix in $\mathrm{Lmx} 4$ and extends far above the root in Lmx3 (Figure 6.2). Important intra-individual variation also occurs along the tooth row in the troodontid Byronosaurus jaffei (MPC-D 100-983), in which some maxillary and dentary crowns are strongly folidont and lack longitudinal ridges and labial depressions (Figure 6.3). Yet, more distal teeth tend to be devoid of constriction between root and crown and display a single, well-visible longitudinal ridge and a deep depression labially (Figure 6.3). Despite these dental variations along the tooth row, isolated theropod teeth can often be assigned to 'family' or 'sub-family' level clades with confidence, some of them up to the genus level, therefore providing important information on the biogeographic and stratigraphic ranges for these taxa and clades, and stratigraphic information for deposits preserving highly diagnostic theropod shed teeth.

\section{Evolutionary Transformations in the Non-Avian Theropod Dentition}

The distribution of 145 dental characters on the theropod general consensus tree allows us to identify several evolutionary transformations within the dentition across Theropoda. These evolutionary steps can be summarized as follow:

Number of teeth. (1) Increase in the number of premaxillary teeth to more than five in Spinosauridae. (2) Decrease in the number of maxillary teeth to fewer than ten in Avialae (i.e., Epidexipteryx + Archaeopteryx), to fewer than 15 in the clade Dilophosaurus + Averostra, carcharodontosaurines, and the clade Microraptorinae + Eudromaeosauria (ACCTRAN for the latter), and to fewer than 20 in pennaraptorans. (3) Increase in the number of maxillary teeth to 15 in allosauroids (ACCTRAN), to more than 14 in proceratosaurids, and to more 


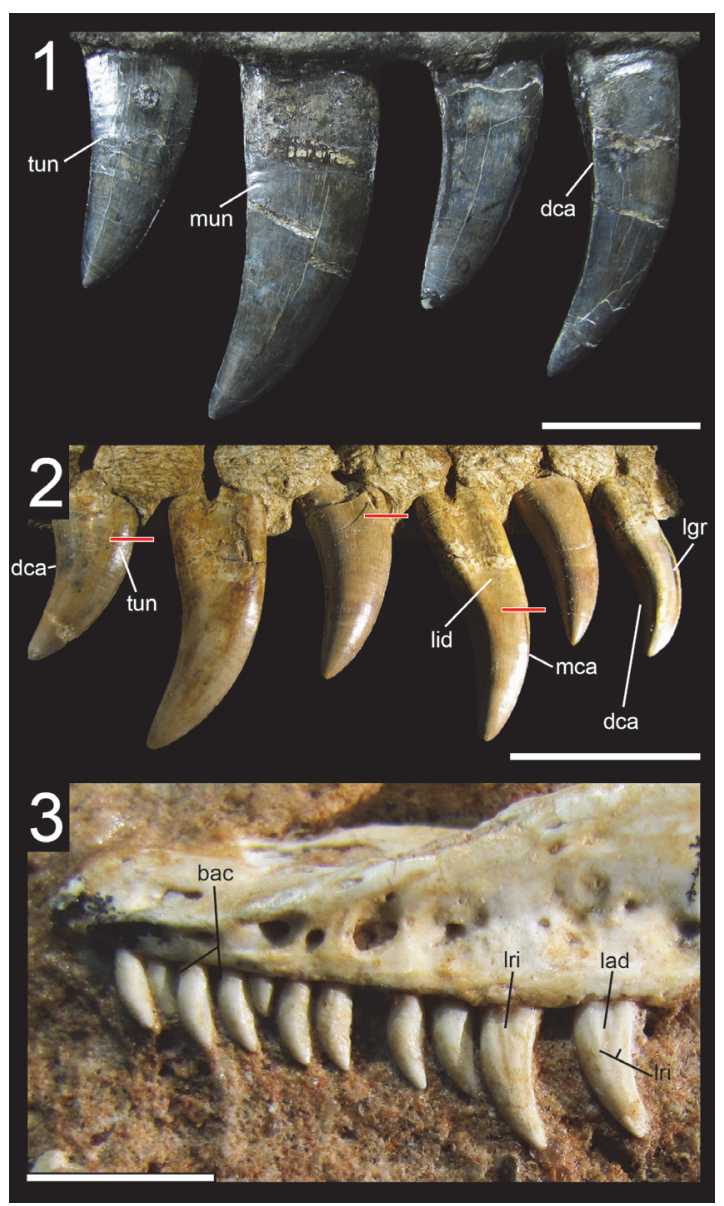

FIGURE 6. Dental variation along the tooth row in nonavian Theropoda. 1, First four right maxillary teeth of the carcharodontosaurid Acrocanthosaurus atokensis (NCSM 14345) in labial view, showing both the presence of marginal and transverse undulations on the third and fourth maxillary teeth, respectively, and a distal carina strongly displaced labially in the first maxillary tooth; 2, First six left maxillary teeth of the tyrannosaurid Alioramus altai (MPC-D 100-1844) in lingual view, showing the strong labial displacement of the distal carina in the three first maxillary teeth, a longitudinal groove, a lingual depression and transverse undulations on the first, third and sixth maxillary teeth, respectively, and the variable basal extension (represented by the horizontal red line) of the mesial carina along the maxillary tooth row; 3, Mesial left maxillary teeth of the troodontid Byronosaurus jaffei (MPC-D 100-983) in labial view, showing the presence and absence of a basal constriction in the mesial and distal maxillary teeth, respectively, a single and two longitudinal ridges on the labial surface of the second and third ziphodont maxillary teeth, respectively, and a labial depression on the third ziphodont maxillary tooth. Abbreviations: bac, basal constriction; dca, distal carina; lad, labial depression; lid, lingual depression; Igr, longitudinal groove; Iri, longitudinal ridge; mca, mesial carina; mun, marginal undulation; tun, transverse undulation. Scale bars $=5$ $\mathrm{cm}(1), 3 \mathrm{~cm}(2), 5 \mathrm{~mm}$ (3). than 19 teeth in maniraptoriforms and derived troodontids (i.e., troodontids more derived than Sinusonasus; ACCTRAN for the latter). (4) Increase in the number of dentary teeth to more than 25 in baryonychines, maniraptoriforms, and troodontids (ACCTRAN for the two latter). (5) Decrease in the number of dentary teeth to fewer than 15 in ceratosaurs (ACCTRAN), megalosaurids, neocoelurosaurs and avialans (ACCTRAN for the two latter), and to fewer than 26 in pennaraptorans (ACCTRAN).

Tooth loss. (1) Loss of distal premaxillary teeth in the clade Caudipteridae + Caenagnathoidea. (2) Loss of premaxillary teeth in Ornithomimosauria more derived than Pelecanimimus, Therizinosauria/Therizinosauroidea and Caenagnathoidea. (3) Loss of distal maxillary teeth in Ornithomimosauria, possibly in Scansoriopterygidae and a basal clade comprising Almas and Jinfengopteryx among Troodontidae. (4) Loss of the mesialmost dentary teeth in Oviraptorosauria and derived Therizinosauroidea, and loss of distal dentary teeth in ornithomimosaurs more derived than Pelecanimimus, possibly also in Scansoriopterygidae. (5) Loss of maxillary and dentary teeth in Ornithomimosauria more derived than Shenzhousaurus and in the clade Caudipteridae + Caenagnathoidea among Oviraptorosauria. (6) Loss of palatal teeth in theropods (ACCTRAN).

Tooth row extension. (1) Distal displacement of tooth-row throughout the evolution of theropods, with the distalmost maxillary tooth: lying posterior to the anterior rim of orbit in non-dilophosaurid and non-averostran theropods (DELTRAN); being anterior or aligned to the anteriormost rim of orbit and posterior to the posteriormost rim of the antorbital fenestra in non-tetanuran averostrans, Liliensternus (unknown) and dilophosaurids (ACCTRAN); being anterior or aligned to the posteriormost rim of the antorbital fenestra and posterior to the anteriormost rim of the antorbital fenestra in non-ornithomimosaur maniraptoriforms and non-troodontid and avialan paravians (ACCTRAN); being aligned to the anteriormost rim of the antorbital fenestra in troodontid and avialan paravians (ACCTRAN). (2) The tooth row even extends anterior to the anteroventral rim of the antorbital fenestra in ornithomimosaurs and possibly alvarezsaurids.

Dentition size. (1) Decrease in crown size in coelurosaurs and noasaurids (ACCTRAN), whose mesial and lateral dentitions include teeth of less than one centimeter in apicobasal height. (2) Increase in crown size in Tyrannosauroidea and Dromaeosauridae more derived than Graciliraptor, 
whose dentition encompasses teeth of more than one centimeter, and Ceratosauridae, Allosauroidea (ACCTRAN) and derived Tyrannosaurinae, which all bear teeth of more than six centimeters. (3) Decrease in size of distal premaxillary teeth in spinosaurids, oviraptorosaurs (ACCTRAN) and microraptorine dromaeosaurids, whose mesial premaxillary teeth are significantly larger than the distal ones. (4) Isodonty (teeth of equal width and close height) of premaxillary and mesial maxillary teeth in Abelisauroidea, mesial premaxillary and maxillary teeth in Spinosauridae, and distal premaxillary and mesial maxillary teeth in Allosauria and Avialae (DELTRAN). (5) Isodonty of maxillary teeth in Paraves (ACCTRAN) or Dromaeosauridae (DELTRAN), and morphometric heterodonty (n.b., here referred to as teeth of different width and height, not shape) in troodontids, whose mesial maxillary teeth are significantly smaller than those from the posterior portion of the maxilla. (6) Decrease in size of mesialmost maxillary teeth in ceratosaurids, tyrannosauroids, and paravians (ACCTRAN), whose mid-maxillary teeth are significantly larger than the mesialmost maxillary teeth. (7) Decrease in size of mesialmost dentary teeth in tyrannosauroids and abelisaurids, whose mid-dentary teeth are significantly larger than mesialmost dentary teeth. There is an increase in size of the mesialmost dentary teeth in Spinosauridae, derived therizinosaurids (i.e., Eshanosaurus + Erlikosaurus), and possibly Scansoriopterygidae. (8) Decrease in size of the first dentary tooth, compared to the second and third dentary teeth, in neotheropods more derived than Coelophysis, and paravians (ACCTRAN). (9) Increase in size of the first dentary tooth, which is equal in size to more distally positioned dentary teeth, in noasaurids, Neocoelurosauria (ACCTRAN), and the sisterclade of the clade encompassing Almas among Troodontidae.

Alveoli. (1) Development of subrectangular alveoli in Abelisauridae, subcircular maxillary alveoli in Spinosauridae and Maniraptoriformes (ACCTRAN), and oval/lenticular maxillary alveoli in Pennaraptora. (2) Merging of alveoli to form an open groove in ornithomimosaurs and possibly alvarezsaurids for the maxilla, and in troodontids and possibly alvarezsaurids for the dentary.

Basal constriction. (1) Mesiodistal constriction at the level of the cervix in mesial teeth in neocoelurosaurs (ACCTRAN) and in carcharodontosaurines, alvarezsaurids and maniraptoriforms (DELTRAN for the two latter) in lateral teeth. (2) Loss of a constriction in the lateral dentition in dromaeosaurids, and in the mesial dentition in non-halszkaraptorine dromaeosaurids (ACCTRAN). (3) Development of an important constriction in the lateral dentition of therizinosaurs.

Basal crown cross-sectional outline. (1) Development of a salinon-shaped cross-section of the crown base in the mesialmost dentition of Abelisauroidea and Allosauroidea, and a U-shaped cross-section in pantyrannosaurians. (2) Development of a D-shaped or J-shaped cross-section of the base-crown in the mesialmost teeth in Dromaeosauridae and Coelurosauria more derived than Bicentenaria. (3) Development of a subcircular cross-section of the base-crown in the mesial dentition of carcharodontosaurids and neocoelurosaurs more derived than Ornitholestes, in the lateral dentition of maniraptoriforms, and in the mesial and lateral dentitions of spinosaurids. (4) Labiolingual constriction of the crown base at midlength of the tooth, creating a figure-8-shaped cross-section of the crown, in the lateral dentition of non-halszkaraptorine dromaeosaurids.

Crown curvature. (1) Loss of a concave distal profile in the lateral dentition of Abelisauridae (ACTRAN) or Abelisauridae (DELTRAN), spinosaurines (ACTRAN) and paravians. (2) Development of a convex distal profile of the lateral crowns in Maniraptoriformes and a concave distal profile of the lateral crowns in Dromaeosauridae.

Denticle/carina loss. (1) Loss of a mesial carina in the mesial and lateral dentitions (ACCTRAN), and loss of distal denticles in the mesial dentition of Neocoelurosauria. (2) Loss of a distal carina in the lateral dentition of Maniraptoriformes (ACCTRAN) or Paraves (DELTRAN). (3) Loss of mesial and distal serrations in the mesial and lateral dentitions of Spinosaurinae. (4) Development of a denticulated distal carina in the mesial dentition of Therizinosauroidea and Eudromaeosauria (ACCTRAN), and in the lateral dentition of troodontids more derived than Byronosaurus, and the clade gathering Microraptorinae and Eudromaeosauria.

Denticle shape. Development of asymmetrically convex or hooked denticles in the lateral dentition of Abelisauridae, Troodontidae more derived than Byronosaurus, and Therizinosauroidea (ACCTRAN).

Distal denticles larger than mesial denticles (DSDI > 1.2). (1) Reduction in size of mesial denticles compared to distal ones in Noasauridae (excluding Limusaurus), Piatnitzkysauridae and Coelurosauria. (2) Increase in size of mesial denticles compared to distal ones in Carcharodontosauridae (ACCTRAN). (3) Increase in the size of 
mesial denticles in adult Tyrannosauroidea more derived than Eotyrannus, and Therizinosauria (ACCTRAN) becoming equally sized as the distal denticles.

Interdenticular sulci. Development, in the lateral dentition, of interdenticular sulci between apical denticles in the mesial carina of Allosauroidea (ACCTRAN) and Megalosaurinae more derived than Duriavenator, and between mid-crown denticles of the distal carina in Piatnitzkysauridae, Megalosauridae more derived than Sciurumimus, Allosauroidea, Tyrannosauroidea (ACCTRAN) and Eudromaeosauria more derived than Graciliraptor.

Carina. (1) Basal extension of the mesial carina to the cervix (or below it) in the mesial dentition of Abelisauroidea, Spinosauridae and Allosauroidea (DELTRAN), and in the lateral dentition of neotheropods more derived than Liliensternus (DELTRAN). (2) Apicobasal shortening of the mesial carina, terminating well-above the cervix, in the lateral dentition of Megalosauroidea, Carcharodontosauria and Coelurosauria more derived than Bicentenaria (ACCTRAN for the two latter).

Enamel undulations. (1) Development of transverse undulations in some lateral crowns in Averostra (ACCTRAN), Megalosaurinae, and Dromaeosauridae more derived than Graciliraptor. (2) Development of pronounced and well-visible transverse undulations in some lateral crowns in Ceratosauria and Allosauroidea (both ACCTRAN). (3) Development of marginal undulations in some lateral crowns in Megalosaurinae more derived than Duriavenator, Tyrannosaurinae more derived than Alioramus, and Orionides (ACCTRAN). (4) Development of mesiodistally elongated marginal undulations in some lateral crowns in Allosauria.

Labial depression. Development of a centrally positioned labial depression extending on the basal half or more than half of the crown in Megaraptora and non-halszkaraptorine Dromaeosauridae.

Flutes. Development of flutes on both labial and lingual surfaces in some lateral teeth in Spinosauridae and Microraptorinae (ACCTRAN).

Enamel surface texture. (1) Development of a braided oriented enamel texture in Microraptorinae + Eudromaeosauria, and a veined enamel texture in Spinosauridae (ACCTRAN). (2) Loss of an oriented enamel texture (to become an irregular and non-oriented texture) in Abelisauroidea (ACCTRAN), Metriacanthosauridae, and Neocoelurosauria more derived than Ornitholestes.

Root. Mesiodistal inflation of the root at mid-height in Compsognathidae and Maniraptora, and apical tapering of the root in Spinosauridae and Therizinosauria (ACCTRAN).

\section{Major Evolutionary Transitions in the Non- Avian Theropod Dentition}

The distribution of dental characters based on our dentition-based data matrix allows us to highlight several major evolutionary trends in theropods.

Spinosauridae. The most important dental evolutionary transition occurred with the radiation of spinosaurid theropods (Figure 3; Table 1). With 21 dental synapomorphies, the spinosaurid dentition is highly specialized, from the conical fluted crowns bearing minute or no denticles at all to the terminal rosette of the upper and lower jaw with subcircular alveoli (e.g., Charig and Milner, 1997; Sereno et al., 1998; Sues et al., 2002; Dal Sasso et al., 2005; Hendrickx and Mateus, 2014a; Sales and Schultz, 2017). Several studies have shown that these theropods were semi-aquatic animals feeding, at least partially, on fish (e.g., Charig and Milner, 1997; Rayfield et al., 2007; Amiot et al., 2010; Ibrahim et al., 2014; Hendrickx et al., 2016; Hassler et al., 2018), suggesting that their peculiar dentition is linked to the specialized piscivorous diet.

Buffetaut (2011) and Serrano-Martínez et al. (2016) investigated the early evolution of the spinosaurid dentition based on isolated teeth from the Jurassic of Tanzania and Niger. According to these authors, dental adaptations towards piscivory were gradually acquired throughout the evolution of Spinosauridae. The teeth of the possible spinosaurid Ostafrikasaurus crassiserratus from the Tendaguru Formation (Late Jurassic) of Tanzania are characterized by large denticles (10 denticles per $5 \mathrm{~mm}$ ), 'wrinkled' enamel texture, and weakly compressed fluted crowns (Buffetaut, 2011; Rauhut, 2011). This suggests that a reduction in denticle size or the disappearance of denticles entirely and a decrease of labiolingual compression (CBR) probably occurred after the Jurassic (Buffetaut, 2011). Likewise, the presence of a single non-fluted spinosaurid tooth (MUPE HB-87) with a veined enamel texture from the Irhazer Group (Middle Jurassic) of Niger suggests that veined texture of the enamel evolved early in spinosaurid evolution, unlike flutes that appeared later (Serrano-Martínez et al., 2016).

The taxonomy of these teeth is, however, questionable. The teeth referred to Ostafrikasaurus have many features present in the mesial teeth of Ceratosaurus such as flutes, large denticles and a moderate labiolingual compression (Rauhut, 2011; C.H. personal obs.). In addition, the fine 'wrinkling' 
described by Buffetaut (2011) is as pronounced as that observed in Ceratosaurus premaxillary teeth (UMNH VP 5278), and the crown even shows a smooth enamel texture on the labial side (Rauhut, 2011). This enamel surface texture differs significantly from the deeply veined or anastomosed enamel texture of spinosaurid crowns (C.H. personal obs.). Given that ceratosaurids were possibly present in the Kimmeridgian-Tithonian of Africa (Janensch, 1925; Rauhut, 2011), and although some teeth of Ostafrikasaurus do show flutes on both labial and lingual surfaces and present a mesial carina reaching the cervix, two features that were not observed in any mesial Ceratosaurus teeth, their similarity with ceratosaurid mesial teeth casts doubt on their spinosaurid affinity. We think that the tooth ascribed to Spinosauridae by Serrano-Martínez et al. (2016) unlikely belongs to this clade. Given the relatively low denticle density ( 17.5 denticles per $5 \mathrm{~mm}$ for MC and DC), a mesial carina terminating far above the cervix, and the braided texture of the enamel (i.e., the texture is not deeply veined, does not curve basally close to carina and strongly resembles the braided texture of megalosaurid teeth; see Hendrickx et al., 2015c; C.H. personal obs.), it is likely that MUPE HB-87 represents a tooth from the mesial dentition of a megalosaurid, most probably Afrovenator abakensis, rather than a spinosaurid.

The evolutionary steps proposed by Buffetaut (2011) and Serrano-Martínez et al. (2016) for the spinosaurid dentition based on putative spinosaurid shed teeth must, therefore, be considered as tentative. Likewise, given the amount of homoplasy in dental features, it is unlikely that the taxonomic attributions in those works are accurate. Although a reduction of denticle size, a decrease in labiolingual crown compression and the development of flutes and veined/anastomosed enamel texture had to occur through Spinosauridae evolution prior to the appearance of definitive spinosaurid taxa, the timing and sequence of these features remain unknown.

Avetheropoda. Two major evolutionary steps in the dentition of theropods occurred during the radiation of Allosauroidea and Tyrannosauroidea (Figures 3 and 4; Table 1). These dental changes affect the mesial and lateral dentitions of allosauroids but are restricted to the mesial dentition in tyrannosauroids. The outgroup condition for allosauroids and coelurosaurs is transversely narrow (i.e., CBR < $0.75)$ mesial teeth, in which the cross-sectional outline at the crown base is lanceolate. The dental evolution of basal avetheropods is marked by a transverse thickening of mesial teeth (CBR between 0.75 and 1.2 ), yet the lanceolate crosssectional outline was retained by basalmost coelurosaurs such as Bicentenaria. Consequently, both allosauroids and coelurosaurs more derived than Bicentenaria independently evolved mesial teeth in which the mesial carina migrated mesiolingually, and concavities appeared adjacent to the mesial and/or distal carinae on the lingual surface of the crown, resulting in a J-shaped and/or salinon-shaped cross-sectional outline at the crown base in mesialmost teeth. Basal tyrannosauroids developed mesial teeth in which the mesial carina twists onto the mesiolingual surface whereas the distal carina moved labially and faces lingually (Figure 4; Table 1). These evolutionary changes in both Allosauroidea and Tyrannosauroidea likely result from an anteroposterior shortening of the premaxilla as well as an adaptation towards a diet involving bone-crunching and bone-biting, with a high degree of torsion applied on the mesial dentition. The mesial teeth of some tyrannosaurids share a high CBR and a relatively small angle between the mesial and distal carinae with basal allosauroids, indicating the ability to endure high mechanical stresses (Reichel, 2010, 2012). The mesial dentition of these groups was specialized for gripping and pulling on the prey, and the lingually deflected carinae of the mesial teeth, which have a U- or D-shaped cross-sectional outline, made them effective tools for defleshing carcasses (Reichel, 2012). The anteroposterior shortening of the premaxilla leads to the overlap of the second and third premaxillary alveoli, a dental feature seen in both Allosauroidea and Tyrannosauroidea. This shortening was accentuated in basal tyrannosauroids, in which the third and fourth premaxillary alveoli also overlap. Similarly, abelisaurid theropods had an anteroposterior shortening of their premaxillae and developed labiolingually thick mesial teeth showing a salinon, J-shaped or Dshaped cross-sectional outline. In Abelisauridae, the premaxillary alveoli remain mesiodistally oriented and do not overlap. In Tyrannosauroidea, these dental changes were accompanied by a decrease in size of the mesialmost dentary teeth. Conversely, allosauroids retained relatively large premaxillary and mesial dentary teeth, as well as a mesial dentition with distally-positioned distal carina and crowns with a strongly convex lingual margin. In Allosauroidea, the mesial and distal denticles remained equal in size, whereas basal tyrannosauroids are characterized by mesial denticles significantly smaller than distal denticles, a 
dental feature present in basal coelurosaurs such as Bicentenaria (Novas et al., 2012) and Zuolong (Choiniere et al., 2010a). The development of interdenticular sulci between distal denticles and transverse undulations in lateral teeth also occurred in allosauroids, whereas the lateral teeth of basal tyrannosauroids resemble those of more basal coelurosaurs.

Neocoelurosauria and the shift in feeding ecology in Maniraptoriformes. The high number of dental synapomorphies diagnosing Maniraptoriformes (five; Figure 4; Table 1) marks an important transition in the evolution of the coelurosaur dentition characterized by tooth simplification. This transition has been suggested to be functionally linked to a switch in dietary preferences (Zanno and Makovicky, 2011; see below). Such dental transformation occurred gradually throughout the evolution of neocoelurosaurs, and changes in tooth morphology seem to have occurred in three evolutionary steps.

(1): Loss of mesial denticles in both mesial and lateral teeth, and loss of distal denticles in mesial teeth in basal neocoelurosaurs. While basalmost coelurosaurs such as Bicentenaria, Zuolong and basal tyrannosauroids have denticulated mesial and distal carinae along the whole dentition, a mesial carina is absent in the mesial and distal dentitions of most compsognathids, and the distal carina is unserrated in the mesial teeth of these taxa. A mesial carina is, nevertheless, present in some mesial and lateral teeth of the basalmost neocoelurosaurs Ornitholestes and Fukuivenator, but mesial denticles are absent and the carina is restricted to the apical portion of the crowns (Azuma et al., 2016; C.H. personal obs.). Sinocalliopteryx, which is a large and likely adult individual, appears to be the only member of Compsognathidae that retained denticles on the distal and mesial carinae in mesial and lateral teeth, respectively ( $\mathrm{Ji}$ et al., 2007). Conversely, Ornitholestes and all compsognathids with the exception of Sinocalliopteryx have unserrated mesial teeth and distally denticulated lateral teeth (Stromer, 1934; Ostrom, 1978; Currie and Chen, 2001; Hwang et al., 2004; Chiappe and Göhlich, 2010; Dal Sasso and Maganuco, 2011; C.H. personal obs.). The basal neocoelurosaur Fukuivenator is characterized by a dentition in which all teeth appear to be fully unserrated (Azuma et al., 2016), an apomorphic condition among neocoelurosaurs given the presence of serrations in compsognathids, Ornitholestes, basal alvarezsauroids, therizinosaurs and oviraptorosaurs (see below). The systematic position of Fukuivenator is still unresolved in Neocoelurosauria and this taxon might be a basal paravian closely related to basal dromaeosaurs such as Halsz- karaptor due to the presence of D-shaped mesial teeth and unserrated crowns. Unlike Fukuivenator, some lateral teeth show denticles restricted to a certain portion of the distal carina in Ornitholestes (AMNH 619), Compsognathus (MNHN CNJ 79; Dal Sasso and Maganuco, 2011), Scipionyx (Dal Sasso and Maganuco, 2011) and Juravenator (JME Sch 200). In these taxa the denticulated distal carina does not reach the cervix and/or the apex, marking a further step towards tooth simplification in coelurosaurs. Furthermore, the lateral dentition of Compsognathus (MNHN CNJ 79; Stromer, 1934; Peyer, 2006) and Ornitholestes (AMNH 619) comprises both finely denticulated (i.e., more than 10 denticles per $1 \mathrm{~mm}$ ) and completely unserrated carinae. Unserrated teeth are present in the mesial maxillary teeth of Scipionyx (Dal Sasso and Maganuco, 2011). The absence of denticles in the mesial and/or distal carinae of Ornitholestes and most compsognathids could be ontogenetic given that these specimens might represent young individuals (e.g., Ostrom, 1978; Currie and Chen, 2001; Hwang et al., 2004; Chiappe and Göhlich, 2010; Dal Sasso and Maganuco, 2011). Yet, given the absence of fully-grown specimens among many basal neocoelurosaurs, and because the subadult specimen of Fukuivenator may represent a basal neocoelurosaur with unserrated mesial and lateral teeth, the absence of carina and denticles in neocoelurosaurs is here considered as a derived dental character.

(2): Conical mesial teeth and irregular enamel surface texture in the clade Compsognathidae + Maniraptoriformes. The subcircular crown cross-section present in the mesial teeth of Compsognathidae, Ornithomimosauria, Alvarezsauroidea and Oviraptorosauria contrasts with the J-shaped, D-shaped and/or U-shaped mesial teeth of the basal coelurosaur Zuolong (Choiniere et al., 2010a), tyrannosauroids (e.g., Holtz, 2004; Rauhut et al., 2010) and the basal neocoelurosaurs Ornitholestes (AMNH 619) and Fukuivenator (Azuma et al., 2016). Nevertheless, the basal ornithomimosaur Pelecanimimus (Pérez-Moreno et al., 1994) and the basalmost therizinosaur Falcarius (UMNH 15097) also show a D-shaped cross-sectional outline at the crown base in some mesial teeth, a dental feature here tentatively considered as autapomorphic to these taxa. It is, however, possible that unserrated mesialmost teeth with a D-shaped cross-section is synapomorphic for a clade excluding Compsognathidae and gathering Ornitholestes, Fukuivenator, and maniraptoriforms, with D-shaped and unserrated mesial teeth being the plesiomorphic condition to basal ornithomimosaurs and therizinosaurs, a hypothesis developed below. The mesial dentition of compsognathids retains the strong distal curvature of basal coelurosaurs but the enamel surface texture of the crown is no lon- 
ger braided and oriented. A braided surface texture of the enamel is clearly present in Bicentenaria, Dilong, Guanlong, Ornitholestes, and Proceratosaurus whereas it is irregular at least in Compsognathus, Haplocheirus, Juravenator, and Nqwebasaurus. A braided enamel surface texture is, however, present in is, however, present in some crowns of the basalmost alvarezsauroid Aorun (IVPP V15709), a possible autapomorphic feature of this taxon.

(3): Increase in the number of maxillary teeth, loss of distal curvature in both mesial and lateral teeth, and lateral teeth with a subcircular outline in Maniraptoriformes. Unlike non-maniraptoriform coelurosaurs, the distal profile of mesial and lateral teeth of basal maniraptoriforms is no longer concave but straight and convex, respectively, and all teeth have a subcircular cross-sectional outline. The combination of these dental features and the presence of a constriction define the conidont/folidont dentition of alvarezsaurids, ornithomimosaurs, oviraptorosaurs and therizinosaurs, which contrasts with the ziphodont dentition of the large majority of non-maniraptoriform theropods. Ziphodont teeth with distally recurved crowns were, however, retained in the lateral dentition of the basal alvarezsauroid Haplocheirus (Choiniere et al., 2014b). The position of Aorun, a coelurosaur bearing ziphodont mesial and distal teeth, at the base of Alvarezsauroidea (Xu et al., 2018), in fact, leads to a single dental synapomorphy for the clade Maniraptoriformes (i.e., the subcircular cross-section outline of lateral teeth) and no dental synapomorphies for Maniraptora. The recent discovery of basal alvarezsauroids with a ziphodont dentition, therefore, suggests that: i) basal ornithomimosaurs also evolved from ziphodont theropods with distally recurved teeth and independently developed straight and minute unserrated crowns; or that ii) basal alvarezsauroids like Aorun and Haplocheirus evolved from taxa with a conidont/folidont dentition and reacquired ziphodont teeth. We favor the first hypothesis given the presence of distal serrations in the lateral dentition of the basalmost therizinosaur Falcarius (Zanno, 2010a) and oviraptorosaur Incisivosaurus (IVPP V13326; see below), and distally recurved lateral teeth in the basal ornithomimosaur Pelecanimimus (LH 777). In addition, Haplocheirus represents the only definitive non-paravian maniraptoriform known from the Jurassic, and particularly long (i.e., >30 Ma) ghost lineages remain to be filled for the clades Ornithomimosauria, Therizinosauria and Oviraptorosauria (n.b., the dental and mandibular anatomy of Eshanosaurus suggests that this taxon is likely a derived therizinosaur from the Cretaceous; Barrett, 2009). Consequently, the basalmost members of ornithomimosaurs, therizinosaurs and oviraptorosaurs may have also had a ziphodont dentition whose teeth independently evolved into conidont or folidont teeth.

The sequence of these steps in the evolution of the neocoelurosaur dentition may change with the potential discovery of basal ornithomimosaurs, therizinosaurs and oviraptorosaurs in Jurassic deposits. If revealed to be a basal neocoelurosaur, Fukuivenator from the Early Cretaceous of Japan (Azuma et al., 2016), in fact, disrupts the succession of these steps. The dentition of this taxon shows D-shaped mesialmost teeth and distally recurved crowns, two plesiomorphic features present in basalmost coelurosaurs and Ornitholestes. Yet, Fukuivenator also bears unserrated teeth, as in ornithomimosaurs, and folidont lateral teeth, as in Pelecanimimus, therizinosaurs and basal oviraptorosaurs. A relatively similar dentition is that of the ornithomimosaur Pelecanimimus, which shares with Fukuivenator the presence of D-shaped mesial teeth, unserrated teeth and folidont crowns. Nevertheless, Pelecanimimus bears a large number of premaxillary, maxillary and dentary teeth, which all seem to be constricted. It is, therefore, possible that basal maniraptoriforms like Pelecanimimus and Falcarius retained D-shaped/spatulate unserrated mesial teeth and distally recurved crowns present in basalmost coelurosaurs and Ornitholestes, and evolved a lateral dentition made of constricted teeth with a sub-circular cross-sectional outline at the crown base. In this scenario, denticles, lost in basalmost maniraptoriforms (i.e., Fukuivenator + maniraptoriforms), would be reacquired in the clade Alvarezsauroidea + Maniraptora (e.g., Aorun, Haplocheirus, Incisivosaurus, and therizinosaurs), and independently lost in alvarezsaurids, caudipterids and paravians.

Choiniere et al. (2014b) note that the mesial dentition of Haplocheirus resembles that of derived alvarezsaurids in lacking serrations and having a mild recurvature, whereas the middle and distal maxillary and dentary teeth share the plesiomorphic morphology of coelurosaurs. Unserrated mesial teeth are actually plesiomorphic for the clade Compsognathidae + Maniraptoriformes. A weak curvature of the mesial teeth is also present in Falcarius, Incisivosaurus and Pelecanimimus, and appears to be synapomorphic for maniraptoriforms. We, consequently, consider the mesial and lateral dentitions of Haplocheirus and Aorun to have a plesiomorphic morphology. Choiniere et al. (2014b) also hypothesized either different rates of dental evolution or different evolutionary intervals in the premaxilla and the maxilla to explain such heterodonty in Haplocheirus. Based on the 
sequence of dental evolution observed in neocoelurosaurs, we favor the second hypothesis and suggest that the mesial tooth crown morphology of neocoelurosaurs changed first and appeared before an increase in tooth number, unlike what was inferred for troodontids (Choiniere et al., 2014b).

A large amount of evidence supports the hypothesis that the dental transition seen in the evolution of neocoelurosaurs results from a trophic shift between carnivory and herbivory (Barrett and Rayfield, 2006; Zanno et al., 2009; Zanno and Makovicky, 2011). Direct evidence shows that compsognathids were carnivorous, feeding on an array of small, medium and possibly large-sized prey items such as fish, lizards, birds, theropods, ornithischians and mammals (e.g., Ostrom, 1978; Currie and Chen, 2001; Hwang et al., 2004; Peyer, 2006; Dal Sasso and Maganuco, 2011; Xing et al., 2012). Gastroliths in compsognathids were also found in Sinosauropteryx and Sinocalliopteryx (Dong and Chen, 2000; Xing et al., 2012), which would suggest a partially herbivorous diet in this clade. The presence of few gastroliths combined with the absence of stones in the stomach content of other compsognathids suggest that these stomach stones, known as geo-gastroliths (sensu Wings, 2007), were ingested accidentally (Xing et al., 2012). On the other hand, the presence of a gastric mill in the basal ornithomimosaurs Nqwebasaurus (De Klerk et al., 2000; Choiniere et al., 2012), Shenzhousaurus (Ji et al., 2003), and in the basal oviraptorosaur Caudipteryx (Ji et al., 1998; Zhou and Wang, 2000), implied by the size, distribution and estimated mass of the gastroliths compared to the mass of the animal (Wings and Sander, 2007; Choiniere et al., 2012), allows confident inference of herbivory in these taxa (Barrett, 2005; Zanno and Makovicky, 2011). An herbivorous lifestyle was also inferred in therizinosaurs and alvarezsaurids on the basis of the development of a large number of morphological features supporting herbivory such as: an edentulous premaxilla; the presence of a beak-like keratinous rhamphotheca; U-shaped and dorsally convex dentary; neck elongation; the reduction in bite force in therizinosaurs (Kirkland et al., 2005; Zanno et al., 2009; Zanno, 2010a; Lautenschlager, 2013, 2014; Lautenschlager et al., 2013); the presence of a dorsally convex dentary; a densely packed dentition; and conical/folidont teeth in both therizinosaurs and alvarezsaurids (Zanno and Makovicky, 2011). Zanno and Makovicky (2011) have shown that a change in dentition morphology in maniraptoriforms was accompanied by the development of a large number of cranial and postcranial adaptations towards herbivory such as an elongation of the neck, the presence of an opisthopubic pelvis (this feature may be correlated with the ventilation system rather than herbivory; Macaluso and Tschopp, 2018) and the elongation of manual unguals (Paul, 1984; Zanno and Makovicky, 2011; Lautenschlager et al., 2013; Lautenschlager, 2014).

\section{DISTRIBUTION OF DENTAL FEATURES IN THEROPODA}

The study of the dental morphology in 160 taxa allows us to propose a list of 34 dental features, which when combined can help identify isolated theropod teeth with additional confidence. The distribution of several of these dental features is illustrated in three phylogenetic trees (Figures 3 , 4,7 and 8 ), a summary figure (Figure 9 ) and three tables (Tables 1, 3 and 4). A list of dental synapomorphies in 40 theropod clades illustrated in Figures 3 and 4 is provided in Table 1 based on our data matrix of 145 dental characters coded in 97 saurischian taxa. Table 3 provides the distribution of each dental feature in non-avian theropods whereas Table 4 lists the most important dental features seen in 31 theropod clades. A phylogenetic tree showing the distribution of each of these features for the mesial and lateral dentitions is also provided in Appendix 6. We finally provide functional properties for each dental feature in Table 3.

Although the theropod dentition varies morphologically through ontogeny (e.g., Carr, 1999; Carr and Williamson, 2004; Rauhut et al., 2012; Araújo et al., 2013), the teeth of post-hatchling individuals were also taken into consideration. The identification of teeth belonging to immature individuals can be difficult as their morphology may resemble that of distantly related taxa due to comparable diet or heterochronic processes (e.g., Rauhut et al., 2012). Nonetheless, little difference in tooth morphology and denticle shape and density have been shown to exist between juvenile and adult individuals of theropods like Coelophysis, Albertosaurus, and Gorgosaurus (Buckley et al., 2010; Buckley and Currie, 2014), and some theropod taxa also bear highly diagnostic teeth that can be referred to young individuals based on tooth size only (C.H. personal obs.).

Dentition-based characters are common in cladistic analyses and are often incorporated to help in assessing the relationships of non-avian theropods. The previous use of these dental characters and a discussion of their scorings are pro- 


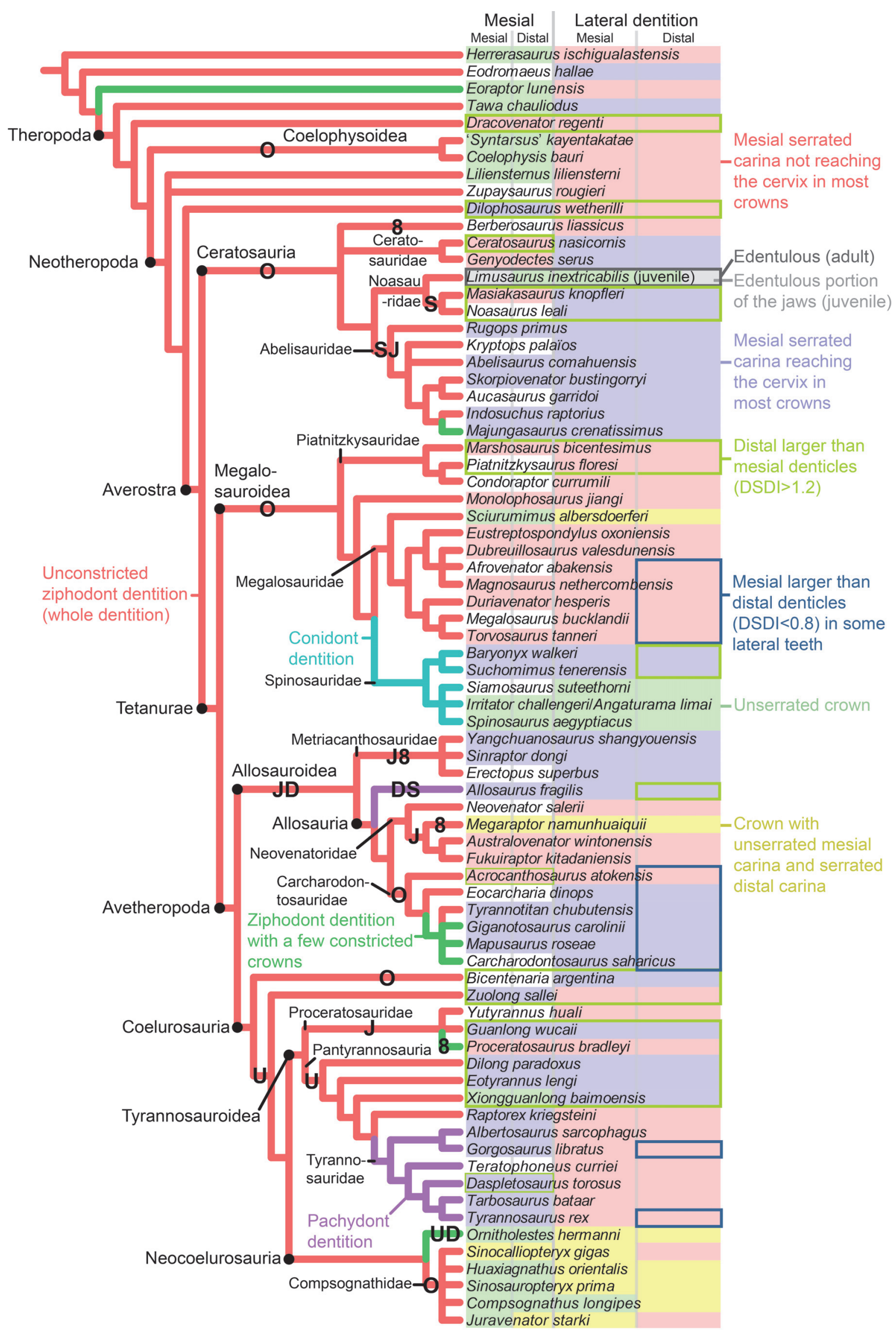


vided in Appendix 7. The datasets, results of the different analyses, and the list of taxa included in this study are provided as .xlsx files in Appendix 8.

\section{Dentition type}

Ziphodonty, a lateral dentition mostly composed of strongly labiolingually narrow crowns with a distal curvature, typically denticulated carinae, and absence of constriction at the cervix (Hendrickx et al., 2015d), is the plesiomorphic condition of theropods, sauropodomorphs or even dinosaurs in general (Sander, 1997; Langer and Benton, 2006; Cabreira et al., 2016; for a different hypothesis see Baron et al., 2017). A ziphodont dentition is present in Ornitholestes, non-alvarezsaurid Alvarezsauroidea, Dromaeosauridae and all non-maniraptoriform theropods other than Spinosauridae, Allosaurus and Tyrannosauridae. The anchiornithine Caihong may also be the only troodontid with a ziphodont dentition. Folidonty, a lateral dentition mostly composed of teeth with an important constriction at the level of the cervix, thus displaying a lanceolate leaf-shaped outline in lateral view (Hendrickx et al., 2015d), is present in Pelecanimimus, therizinosaurs, oviraptorosaurs other than Caudipteryx, Troodontidae (with the possible exception of the anchiornithine Caihong), and some Microraptor specimens. Only Allosaurus and mature Tyrannosauridae display a pachydont dentition (see section on Crown base ratio greater than 0.75), a lateral dentition mostly composed of labiolingually expanded and distally recurved crown, in which the labiolingual width is greater than $60 \%$ of the mesiodistal length, from cervix to apex (Hendrickx et al., 2015c; C.H. personal obs.). Finally, conidonty, a lateral dentition mostly composed of conical crowns bearing minute denticles or no denticles at all (Hendrickx et al., 2015d), is present in all Spinosauridae, all toothed ornithomimosaurs with the exception of Pelecanimimus, the basal oviraptorosaur Caudipteryx (IVPP V12430, NGMC 97-4-A), and the unenlagiine Austroraptor (Novas et al., 2009; Currie and Carabajal, 2012). A conidont mesial dentition, however, characterizes the basal members of the clades Oviraptorosauria (i.e., Incisivosaurus, Caudipteridae) and Scansoriopterygidae, while an unserrated subconidont/ incisiform mesial dentition with a D-shaped crosssection characterizes some basal maniraptoriforms such as Falcarius, Fukuivenator and Ornitholestes (see section on D-shaped cross-section).

Remarks and synapomorphy. Most theropods are pseudoheterodont, characterized by a dentition where crown morphology gradually changes along the jaw so that mesial and lateral teeth differ significantly in their morphology (Hendrickx et al., 2015d). The basal saurischian Eoraptor (Sereno et al., 2013), the noasaurid Masiakasaurus (based on UA 8680 and the referred isolated lateral teeth), the basal maniraptoriform Fukuivenator (Azuma et al., 2016), the microraptorines Microraptor (Xu et al., 2000; Pei et al., 2014) and IVPP V13476 (Xu and $\mathrm{Li}, 2016$ ), and the troodontids Byronosaurus (Makovicky et al., 2003), Daliansaurus (Shen et al., 2017a), IVPP V20378, Jinfengopteryx (Ji et al., 2005), Gobivenator (Tsuihiji et al., 2014), Sinovenator (IVPP V12615), Sinusonasus (IVPP V11527) and Xixiasaurus (Lü et al., 2010) possess a hetero-

FIGURE 7 (previous page). Distribution of dental features in non-neocoelurosaur Theropoda. Phylogenetic tree based on the results obtained by Langer et al. (2017), for non-neotheropod Saurischia, Ezcurra (2017) and Wang et al. (2017a) for non-averostran Neotheropoda, Rauhut and Carrano (2016) and Wang et al. (2017a) for Ceratosauria, Carrano et al. (2012) and Rauhut et al. (2016) for non-coelurosaurian Tetanurae, Brusatte and Carr (2016) for Tyrannosauroidea, and Novas et al. (2012), Rauhut et al. (2012) and Choiniere et al. (2014a) for the phylogenetic distribution of Bicentenaria, Sciurumimus and Zuolong, respectively. The branch colors represent the dentition types and the presence or absence of constricted crowns: ziphodont taxa with unconstricted crowns are in red, ziphodont taxa with a few constricted crowns are in green, conidont taxa are in turquoise, and pachydont taxa are in violet. The colors of taxa represent the presence or absence of serrations on the mesial and distal carinae for both mesial (left column) and lateral dentition (right column): toothless taxa are in grey, taxa with unserrated crowns are in green, taxa with a denticulated distal carina and a denticulated mesial carina not reaching the cervix are in red, taxa with a denticulated distal carina and a denticulated mesial carina reaching the cervix are in blue, and taxa with a denticulated distal carina and an unserrated mesial carina are in yellow. Taxa whose dentition is not known are on a white background. Taxa with distal denticles larger than mesial ones are boxed in green. Some compsognathid taxa possess a double condition in their mesial and lateral dentition: Juravenator bears mesial crowns with denticulated and unserrated distal carina, Compsognathus shows lateral crowns with unserrated and denticulated distal carina, and Sinocalliopteryx possesses denticulated and unserrated mesial carinae in the lateral teeth. Abbreviations: 8, figure-8-shaped crosssection of lateral teeth; D, D-shaped cross-section of mesial teeth; J, J-shaped cross-section of mesial teeth; O, subcircular/lanceolate cross-section of mesial teeth; S, Salinon-shaped cross-section of mesial teeth; U, U-shaped crosssection of mesial teeth. 


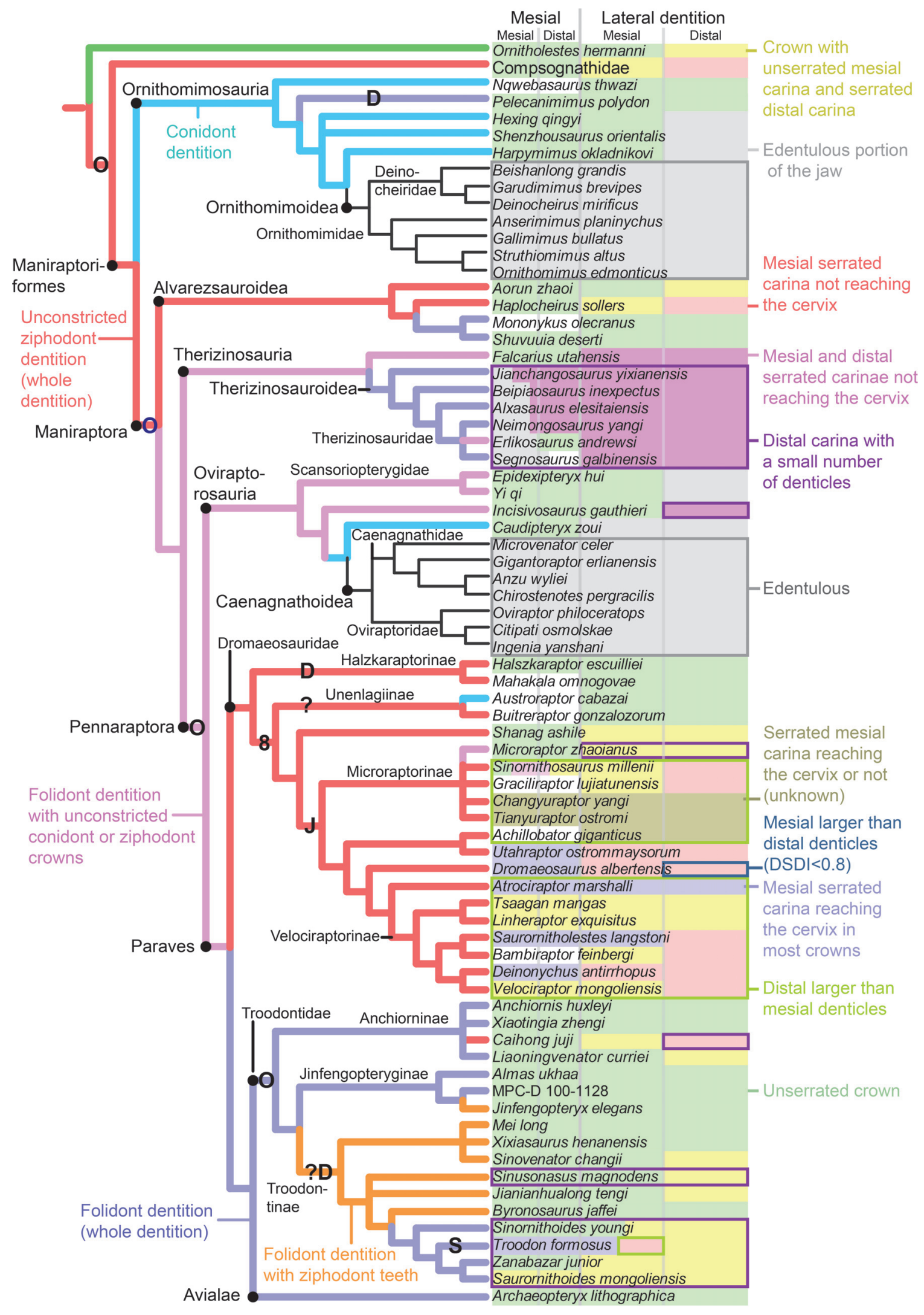


dont lateral dentition that encompasses both ziphodont and folidont teeth. Such heterodont dentition is here considered synapomorphic for Troodontinae (Figure 8). Most lateral teeth of these troodontids appear to be slightly to strongly constricted, thus having a folidont dentition. If a ziphodont dentition, directly associated with faunivory, is the ancestral condition of theropods (e.g., Langer and Benton, 2006; Cabreira et al., 2016), a conidont dentition is synapomorphic for Spinosauridae and Ornithomimosauria. A pachydont dentition is synapomorphic for Tyrannosauridae (see section on Crown base ratio greater than 0.75 ), whereas a folidont dentition is a synapomorphy of Alvarezsauridae/Parvicursorinae and the clade Therizinosauria + Pennaraptora.

Functional morphology. A ziphodont dentition is an adaptation for carnivory, being suited to slashing, cutting through flesh and defleshing (Abler, 1992; Schwenk, 2000; D'Amore, 2009; D'Amore and Blumenschine, 2009; Brink et al., 2015). Folidonty is related to herbivory (Zanno and Makovicky, 2011; Pu et al., 2013) and omnivory (Holtz et al., 1998; Barrett, 2000; Longrich, 2008) as leaf-shaped teeth are suitable for puncturing, tearing apart and shredding fibrous plant fodder and/or small prey (Reisz and Sues, 2000; Sues, 2000). A strictly carnivorous diet has been proposed for derived troodontids with folidont teeth (Zanno and Makovicky, 2011; Torices et al., 2018), yet a folidont dentition appears to be characteristic of herbivorous and/or omnivorous extant animals. Among lepidosaurs, small constricted crowns with a low number of relatively large mesial and distal denticles, as seen in therizinosaurids and derived troodontids, is clearly present in herbivores such as Sauromalus and Iguana (Melstrom, 2017).
Insectivorous and omnivorous lacertilians like Corytaphanes and Ctenosaura also show small teeth with a constriction between crown and root, yet they all appear to be multicusped (i.e., a crown with a low number $(<5)$ of large and prominent convexities of different size, the apicalmost one being the largest), such tooth morphology is absent in theropods. Several lines of evidence, including bite marks on a ceratopsian bone (Jacobsen, 1997), shed teeth associated with ornithopod remains (Ryan et al., 1998), crown microstructure (Brink et al., 2015) and microwear pattern combined with Finite Element Analysis (FEA) (Torices et al., 2018), support the fact that at least the troodontid Troodon was a carnivore and a predator feeding on smaller or softer prey items such as invertebrates (Torices et al., 2018). Consequently, other closely related troodontids may have also had a strictly carnivorous diet (see sections on hooked denticles and small number of denticles on the carina). This is the case of folidont troodontids with large apically pointed denticles like Zanabazar or with a lateral dentition combining folidont and ziphodont teeth such as Byronosaurus, Sinusonasus, Gobivenator and Xixiasaurus [see Lü et al. (2010) for a different opinion].

The crowns of both pachydont and conidont dentitions are particularly labiolingually thick, with pachydont dentitions being present in apex predators adapted to bone-crunching and bone-biting involving a high degree of torsion (e.g., Holtz, 2003; Snively et al., 2006; Holtz, 2008; Reichel, 2010). Conversely, conidont dentitions are adapted to impaling and holding prey items (e.g., Charig and Milner, 1997; Holtz, 1998a; Sereno et al., 1998; Sues et al., 2002; Holtz et al., 2004; Xing et al., 2013b). Besides strengthening the tooth to

FIGURE 8 (previous page). Distribution of dental features in Neocoelurosauria. Phylogenetic tree of Cau et al. (2017) based on the dataset of Brusatte et al. (2014) for Neocoelurosauria, with changes brought by Lamanna et al. (2014) for Caenagnathoidea, and Xu et al. (2018) and Pu et al. (2013) for the phylogenetic position of Aorun and Jianchangosaurus, respectively. The branch colors represent the dentition types: ziphodont taxa with unconstricted crowns are in red, ziphodont taxa with a few constricted crowns are in green, taxa with both folidont and ziphodont lateral dentition are in orange, folidont taxa with unconstricted mesial crowns are in pink, folidont taxa with constricted crowns only are in blue, and conidont taxa are in turquoise. Colors of taxa represent the presence or absence of serrations on the mesial and distal carinae for both mesial (left) and lateral dentition (right): toothless taxa are in grey, taxa with unserrated crowns are in green, taxa with a denticulated distal carina and an unserrated mesial carina are in yellow, taxa with denticulated mesial and distal carinae are in red, and taxa with both denticulated mesial and distal carinae not reaching the cervix are in blue. Taxa (Sinocalliopteryx, Compsognathus and Juravenator) showing both conditions (e.g., mesial dentition with unserrated teeth and lateral dentition with denticulated teeth) are bicolored. Taxa whose dentition is not known are on a white background. Some paravians such as Troodon, Velociraptor and Saurornitholestes possess a lateral dentition with denticulated and unserrated carinae. Taxa with distal denticles larger than mesial ones are boxed in green, and taxa with large typically hooked denticles are boxed in purple. Abbreviations: 8 , figure-8-shaped cross-section of lateral teeth; D, D-shaped cross-section of mesial teeth; J, J-shaped cross-section of mesial teeth; O, subcircular/lanceolate cross-section of mesial (black 'O') and lateral (blue 'O') teeth; U, U-shaped cross-section of mesial teeth. 
Af

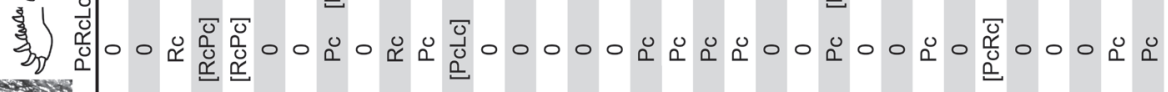

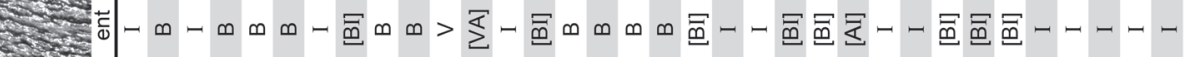
W Indent

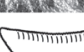

NimIIIIII

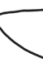

trongy

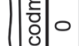

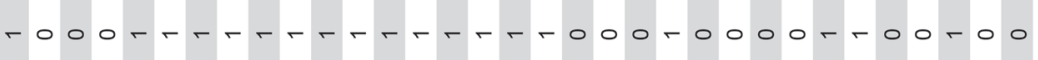

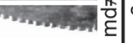

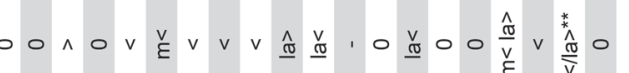

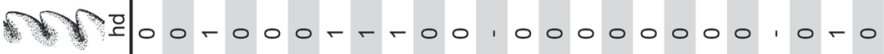

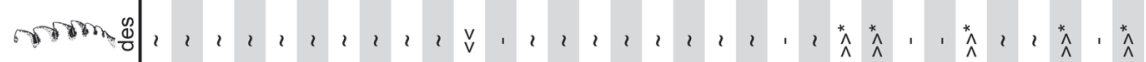

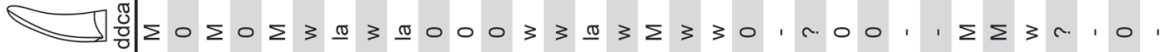

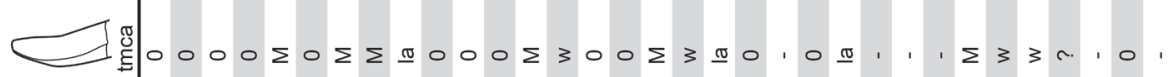

umm $<$ ह

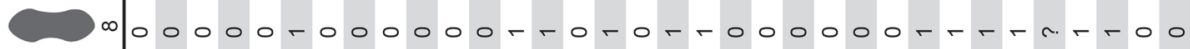

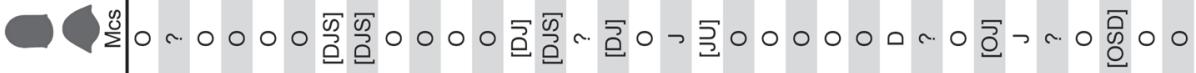
III:0

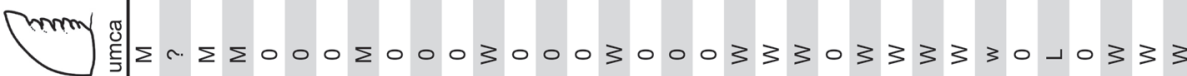

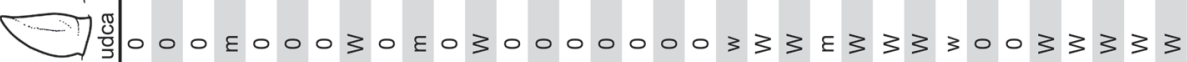

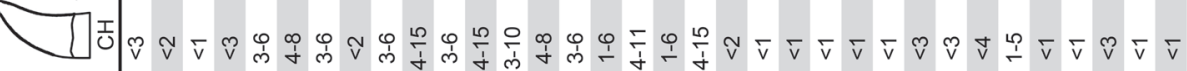

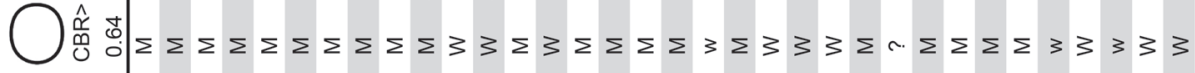

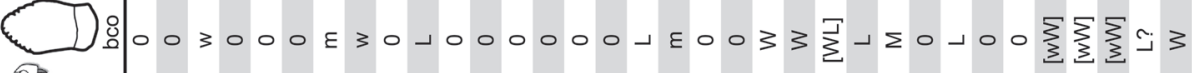

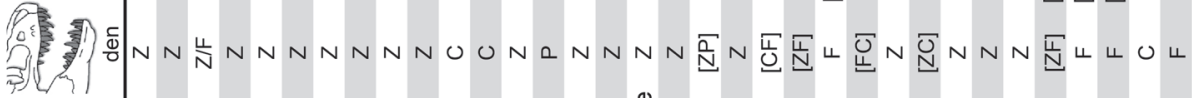

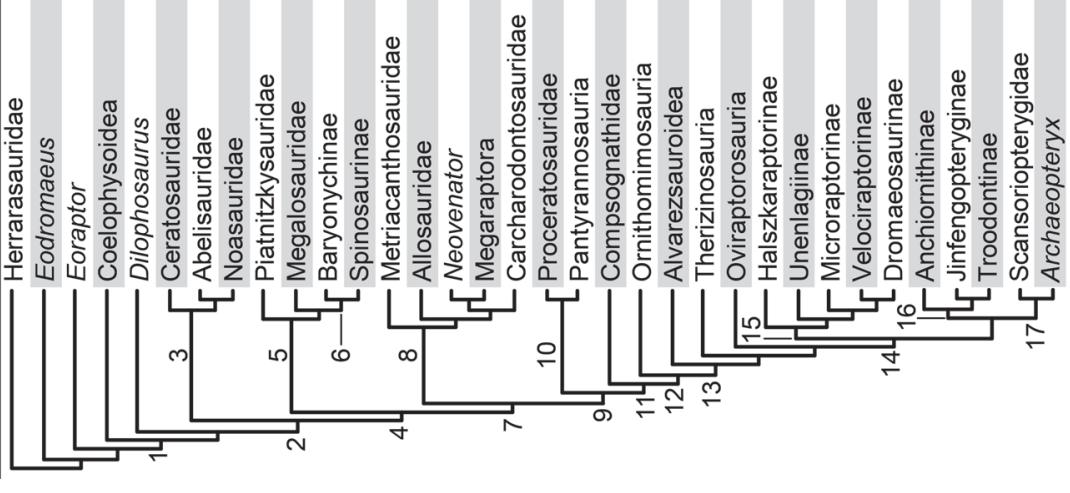


resist lateral bending during feeding, Reichel (2012) also suggested that pachydont teeth provided the ability of individual crowns to make wide cuts through meat. Conidont teeth are often used to infer a piscivorous diet (Baszio, 1997; Sankey, 2001; Brinkman, 2008). The presence of gastroliths in basal ornithomimosaurs and the oviraptorosaur Caudipteryx with minute conical teeth suggests that such dentition was also suited for herbivory (Makovicky et al., 2004; Zanno and Makovicky, 2011; Choiniere et al., 2012). It has also been suggested that the presence of small conical teeth in the mesial dentition of therizinosaurs and Incisivosaurus and the whole dentition of ornithomimosaurs and Caudipteryx is likely a functional precursor of a rhamphotheca, which is present in more derived forms of ornithomimosaurs, therizinosaurs and oviraptorosaurs (Zanno and Makovicky, 2011). The heterodont dentition displayed by some coelurosaurs has been interpreted as an indicator of a dietary shift from faunivory to herbivory or omnivory (Zanno and Makovicky, 2011). A heterodont dentition with simple conical teeth in the mesial portion of the jaws, in fact, characterizes non-carnivorous lepidosaurs such as iguanids (Barrett, 2000; Melstrom, 2017).

\section{Basal constriction of the crown}

Whole dentition. A mesiodistal constriction at the cervix is present in both mesial and lateral teeth in the ornithomimosaur Pelecanimimus (LHC 7777), many therizinosaurs such as Alxasaurus (Russell and Dong, 1993), Eshanosaurus (Xu et al., 2001; Figure 10.3), and Jianchangosaurus ( $\mathrm{Pu}$ et al., 2013), the alvarezsaurid Shuvuuia (MPC-D 100977), basal avialans such as some Archaeopteryx specimens (e.g., Rauhut et al., 2018; Kundrát et al., 2019) and troodontids (e.g., Currie et al., 1990; Baszio, 1997; Norell et al., 2000; Currie and Dong, 2001b; Sankey et al., 2002; Averianov and Sues,

FIGURE 9 (previous page). Distribution of dental features in non-avian Theropoda. Phylogenetic tree based on Hendrickx et al. (2015b) and Cau et al. (2017), with the exclusion of megaraptorans here placed among Neovenatoridae (Benson et al., 2010; Carrano et al., 2012). Letters between brackets represent polymorphic features. Clade numbers: 1, Neotheropoda; 2, Averostra; 3, Ceratosauria; 4, Tetanurae; 5, Megalosauroidea; 6, Spinosauridae; 7, Avetheropoda; 8, Allosauroidea; 9, Coelurosauria; 10, Tyrannosauroidea; 11, Neocoelurosauria; 12, Maniraptoriformes; 13, Maniraptora; 14, Paraves; 15, Dromaeosauridae; 16, Troodontidae; 17, Avialae. Abbreviations: 0, absent; 1, present at least in some teeth or some taxa; 8, figure-8-shaped cross-section at the cervix; ?, unknown; -, inapplicable; , medium-sized denticles (i.e., between 15 and 250 denticles on the carina); $\neq$, difference between mesial and distal denticles; <, distal denticles significantly larger than mesial ones (DSDI > 1.2); $<<$, minute denticles (more than 250 denticles on the carina); >, mesial denticles significantly larger than distal ones (DSDI < 0.9); >>, large denticles (i.e., fewer than 15 denticles on the carina); $\mathbf{A}$, anastomosed texture; $\mathbf{B}$, braided texture; bco, basal constriction at the cervix; bst, basal striations; $\mathbf{C}$, conidonty (dentition with conical crowns); CBR, crown base ratio; $\mathbf{C H}$, crown height in the largest teeth, in centimeters; codm, convex distal margin; cos, concave surface adjacent to carinae; D, D-shaped cross-section; ddca, displaced distal carina; dd, distal denticles; den, dentition type; des, denticle size; dt, present in the dentary; ent, enamel texture; edj, edentulous jaw; F, folidonty (dentition with lanceolate crowns); flu, fluted teeth; hd, hooked denticles; I, smooth or irregular non-oriented texture; ids, interdenticular sulci; J, J-shaped cross-section; L, present in all lateral teeth and, for the edentulism, edentulous posterior portion of the maxilla and/or dentary; la, present in some lateral teeth (e.g., la>, mesial denticles significantly larger than distal ones in some lateral teeth; la-Iri, longitudinal ridge present in some lateral teeth); Lc, presence of laterocumbent teeth; Igr, longitudinal groove; Iri, longitudinal ridges; M, present in all mesial teeth and, for the edentulism, edentulous premaxilla and anterior portion of the dentary; $\mathbf{m}$, present in some mesial teeth (e.g., $\mathbf{m}<$, distal denticles significantly larger than mesial ones in some mesial teeth; $\mathbf{m}$-Igr, longitudinal groove present in some mesial teeth); Mcs, mesial teeth, cross-sectional outline at the cervix; md, mesial denticles; mde, mesial denticles reaching the cervix; mun, marginal undulations; $\mathbf{m x}$, present in the maxilla; $\mathbf{O}$, subcircular/lanceolate cross-section; $\mathbf{P}$, pachydonty (dentition with particularly thick blade-shaped crowns); Pc, presence of procumbent teeth; PcRcLc, procumbent, retrocumbent and laterocumbent dentition; S, Salinon-shaped cross-section; pm, present in the premaxilla; Rc, presence of retrocumbent teeth; tmca, twisted mesial carina; tun, transverse undulations; U, U-shaped cross-section; udca, unserrated distal carina; umca, unserrated mesial carina; V, veined texture; $\mathbf{W}$, present in the whole dentition and, for the edentulism, fully edentulous jaws; $\mathbf{w}$, present in some mesial and lateral teeth; Z, ziphodonty (dentition with blade-shaped crowns). ${ }^{*}$, this applies to derived therizinosaurs and troodontids given that the basal members of these clades either have no serrations at all (e.g., Anchiornis) or minute denticles (e.g., Falcarius, Sinovenator). As for Oviraptorosauria, this applies to the lateral dentition of Incisivosaurus given that caudipterids have unserrated teeth; ${ }^{* *}$, the dentition of basal tyrannosauroids have a DSDI > 1.2 whereas some tyrannosaurid teeth have a DSDI < 0.9. Images of unserrated tooth (udca) and unserrated mesial carina (umca) by Jaime Headden, denticle size (des) and hooked denticles (hd) from Currie et al. (1990, modified), and mesial denticles smaller than distal ones ( $m d \neq d d)$ from Ostrom (1969; modified). 
TABLE 3. Distribution of dental features in non-avian theropods and their hypothetical functionality, adaptation or causes.

\begin{tabular}{|c|c|c|}
\hline Dental feature & Distribution & $\begin{array}{l}\text { Possible functionality, adaptation } \\
\text { or causes }\end{array}$ \\
\hline Ziphodonty & $\begin{array}{l}\text { Ornitholestes, non-alvarezsaurid Alvarezsauroidea, } \\
\text { Dromaeosauridae, and non-maniraptoriform theropods other than } \\
\text { Allosaurus, Austroraptor, Spinosauridae and Tyrannosauridae }\end{array}$ & $\begin{array}{l}\text { Slashing, cutting flesh and defleshing } \\
\text { (carnivory) }\end{array}$ \\
\hline Folidonty & $\begin{array}{l}\text { Pelecanimimus and all other Maniraptoriformes other than } \\
\text { Ornitholestes, Aorun, Haplocheirus, Ornithomimosauria and } \\
\text { Dromaeosauridae }\end{array}$ & $\begin{array}{l}\text { Puncturing, tearing apart and } \\
\text { shredding fibrous plant fodder and/or } \\
\text { small prey (herbivory/omnivory or } \\
\text { carnivory) }\end{array}$ \\
\hline Pachydonty & Allosaurus and mature Tyrannosauridae & Bone-crunching (hypercarnivory) \\
\hline Conidonty & $\begin{array}{l}\text { Spinosauridae, Ornithomimosauria (Pelecanimimus excluded), } \\
\text { Caudipteridae and Austroraptor }\end{array}$ & $\begin{array}{l}\text { Piercing and impaling slippery prey } \\
\text { (piscivory), or raking vegetation } \\
\text { (herbivory); precursor of a } \\
\text { rhamphotheca }\end{array}$ \\
\hline Basal constriction & $\begin{array}{l}\text { Chilesaurus, Pelecanimimus and non-dromaeosaurid Maniraptora, } \\
\text { with the exclusion of non-alvarezsaurid Alvarezsauroidea, } \\
\text { Oviraptorosauria and some Therizinosauria such as Falcarius and } \\
\text { Erlikosaurus for the whole dentition; Masiakasaurus, Majungasaurus, } \\
\text { Chenanisaurus, Proceratosaurus, Ornitholestes and Halszkaraptor for } \\
\text { mesial teeth; Carcharodontosaurinae, Microraptor, Fukuivenator, } \\
\text { Falcarius, Erlikosaurus, Incisivosaurus/Protarchaeopteryx and } \\
\text { possibly Scansoriopterygidae for lateral teeth }\end{array}$ & Crown strength? \\
\hline $\mathrm{CBR}>0.75$ & $\begin{array}{l}\text { Spinosauridae, Allosauridae, Tyrannosauridae, Ornithomimosauria, } \\
\text { Alvarezsauroidea, Therizinosauria, and Troodontidae for both mesial } \\
\text { and lateral teeth }\end{array}$ & $\begin{array}{l}\text { Crown strength (resisting higher } \\
\text { stresses in different directions) }\end{array}$ \\
\hline $\mathrm{CHR}>60 \mathrm{~mm}$ & $\begin{array}{l}\text { Non-neocoelurosaur Averostra with the exclusion of Abelisauroidea, } \\
\text { Piatnitzkysauridae and possibly Baryonychinae, Neovenatoridae and } \\
\text { Proceratosauridae }\end{array}$ & Allometric feature \\
\hline Unserrated crown & $\begin{array}{l}\text { Limusaurus, Spinosaurinae and Maniraptoriformes other than non- } \\
\text { alvarezsaurid Alvarezsauroidea, Therizinosauria, Incisivosaurus, } \\
\text { derived Troodontidae, Microraptorinae, Eudromaeosauria and } \\
\text { possibly Epidexipteryx for the whole dentition; Sciurumimus, some } \\
\text { Coelophysoidea, basal Tyrannosauroidea and Microraptorinae, and } \\
\text { most Compsognathidae for mesial teeth; Aorun, Ornitholestes and } \\
\text { some Compsognathidae and Dromaeosauridae for lateral teeth }\end{array}$ & Crown simplification \\
\hline $\begin{array}{l}\text { Unserrated mesial } \\
\text { carina, serrated distal } \\
\text { carina }\end{array}$ & $\begin{array}{l}\text { Many theropods from most clades for mesial teeth; Sciurumimus, } \\
\text { some megaraptorans, Ornitholestes, Compsognathidae, basal } \\
\text { Alvarezsauroidea, a few Dromaeosauridae and many derived } \\
\text { Troodontidae for lateral teeth }\end{array}$ & Crown simplification \\
\hline $\begin{array}{l}\text { Concave surface } \\
\text { adjacent to carinae }\end{array}$ & $\begin{array}{l}\text { Abelisauroidea, Allosaurus, Tyrannosauroidea and many Paraves for } \\
\text { mesial teeth; some non-neotheropod Saurischia, Ceratosauridae, } \\
\text { non-spinosaurid Megalosauroidea, Metriacanthosauridae, } \\
\text { Neovenatoridae and Therizinosauria for lateral teeth }\end{array}$ & $\begin{array}{l}\text { Increase in enamel surface, better } \\
\text { penetration and withdrawal }\end{array}$ \\
\hline $\begin{array}{l}\text { J-shaped cross- } \\
\text { section }\end{array}$ & $\begin{array}{l}\text { Abelisauroidea, basal Tyrannosauroidea, most non- } \\
\text { carcharodontosaurid Allosauroidea, and some Eudromaeosauria for } \\
\text { mesial teeth; Masiakasaurus and some Troodontidae for lateral teeth }\end{array}$ & $\begin{array}{l}\text { Crown strength with linguodistal } \\
\text { cutting edges }\end{array}$ \\
\hline $\begin{array}{l}\text { Salinon-shaped } \\
\text { cross-section }\end{array}$ & $\begin{array}{l}\text { Abelisauroidea, Allosauridae and some Troodontidae for mesial teeth; } \\
\text { some Troodontidae for lateral teeth }\end{array}$ & $\begin{array}{l}\text { Crown strength with mesiodistal } \\
\text { cutting edges }\end{array}$ \\
\hline $\begin{array}{l}\text { D-shaped cross- } \\
\text { section }\end{array}$ & $\begin{array}{l}\text { Metriacanthosauridae, Allosauridae, some Noasauridae, } \\
\text { Abelisauridae and Megaraptora, Ornitholestes, Pelecanimimus, } \\
\text { Halszkaraptor? and some Troodontidae for mesial teeth }\end{array}$ & $\begin{array}{l}\text { Crown strength with mesiodistal } \\
\text { cutting edges }\end{array}$ \\
\hline $\begin{array}{l}\text { U-shaped cross- } \\
\text { section }\end{array}$ & Zuolong, Ornitholestes and most Pantyrannosauria for mesial teeth & $\begin{array}{l}\text { Crown strength with lingual cutting } \\
\text { edges }\end{array}$ \\
\hline $\begin{array}{l}\text { Figure-8-shaped } \\
\text { cross-section }\end{array}$ & $\begin{array}{l}\text { Berberosaurus, Metriacanthosauridae, some Megaraptora, } \\
\text { Tyrannosauroidea and Troodontidae, and most Dromaeosauridae }\end{array}$ & $\begin{array}{l}\text { Crown strength, better penetration } \\
\text { and withdrawal? }\end{array}$ \\
\hline
\end{tabular}


TABLE 3 (continued).

\begin{tabular}{|c|c|}
\hline Dental feature & Distribution \\
\hline $\begin{array}{l}\text { Mesial carina } \\
\text { reaching the cervix }\end{array}$ & $\begin{array}{l}\text { In some, if not all, mesial and/or lateral teeth of non-averostran } \\
\text { Saurischia, Ceratosauria, Piatnitzkysaurus, Torvosaurus, } \\
\text { Spinosauridae, Allosauroidea (excluding most Neovenatoridae), } \\
\text { Pantyrannosauria, Eudromaeosauria and Troodon }\end{array}$ \\
\hline Twisted mesial carina & $\begin{array}{l}\text { Dilophosaurus, Masiakasaurus, Allosauroidea, basal } \\
\text { Tyrannosauroidea, and some Abelisauridae and Dromaeosauridae fo } \\
\text { mesial teeth only; Piatnitzkysaurus, Allosauridae, and some } \\
\text { Tyrannosauroidea, Therizinosauria and Dromaeosauridae for lateral } \\
\text { teeth }\end{array}$ \\
\hline Split carina & $\begin{array}{l}\text { Allosauroidea, Tyrannosauroidea, Segnosaurus, and } \\
\text { Dromaeosauridae }\end{array}$ \\
\hline $\begin{array}{l}\text { Distal carina strongly } \\
\text { deflected labially }\end{array}$ & $\begin{array}{l}\text { Ceratosauridae, Masiakasaurus, Allosaurus, Sinraptor, } \\
\text { Tyrannosauroidea and Dromaeosaurus for the mesial and some } \\
\text { lateral teeth; Monolophosaurus, Deinonychus, Richardoestesia and } \\
\text { several basal Saurischia, Carcharodontosauria and Dromaeosaurida } \\
\text { for mesial teeth; Saltriosaurus, Berberosaurus, Arcovenator, } \\
\text { Piatnitzkysauridae and Neovenatoridae for some lateral teeth }\end{array}$ \\
\hline Hooked denticles & $\begin{array}{l}\text { Eoraptor, Gojirasaurus, Abelisauroidea, Therizinosauroidea, } \\
\text { Eudromaeosauria, Troodontidae (including Anchiornithinae) }\end{array}$ \\
\hline $\begin{array}{l}\text { Small number of } \\
\text { large denticles on the } \\
\text { carina }\end{array}$ & $\begin{array}{l}\text { Therizinosauroidea, Troodontidae, Incisivosaurus, Microraptor and } \\
\text { Paronychodon }\end{array}$ \\
\hline $\begin{array}{l}\text { Large number of } \\
\text { minute denticles on } \\
\text { the carina }\end{array}$ & Baryonychinae \\
\hline $\begin{array}{l}\text { Sporadic variation of } \\
\text { denticle size }\end{array}$ & Baryonychinae, Nuthetes, Segnosaurus and Richardoestesia \\
\hline
\end{tabular}

Bilobate denticles Abelisauridae, Megalosauridae, Erectopus, Carcharodontosauridae, Tyrannosauridae

Distal denticles larger Non-averostran theropods, Ceratosaurus, Noasauridae,

than mesial denticles Abelisauridae, Piatnitzkysauridae, Baryonychinae, Allosaurus, Acrocanthosaurus, basal Coelurosauria, non-tyrannosaurid Tyrannosauroidea, juvenile Tyrannosauridae, non-unenlagiine Dromaeosauridae, and Troodontinae

Convex distal profile Abelisauridae, Ceratosaurus, Spinosaurinae, Ornithomimosauria, of the crown Alvarezsauroidea, Therizinosauria, and Oviraptorosauria

Fluted crowns Tawa, Coelophysis (juveniles), Masiakasaurus, Spinosauridae, an undescribed metriacanthosaurid and Austroraptor for most of/all the dentition; Ceratosaurus, Scipionyx and Velociraptor for the mesial dentition; Sinosaurus, Dilong and most Microraptorinae for the lateral dentition

Transverse undulations

Eodromaeus, Dracovenator, non-neocoelurosaur Averostra, Falcarius, many eudromaeosaurians and a few derived troodontids; non-neocoelurosaur Averostra when numerous and covering most of the crown

Marginal undulations Ceratosaurus, Abelisauridae, non-neocoelurosaur Tetanurae

Interdenticular sulci Tawa, non-neocoelurosaur Averostra, Falcarius, many eudromaeosaurians and a few derived troodontids

Longitudinal ridges Allosaurus, Tyrannosauroidea, Microraptorinae for mesial teeth; Orkoraptor, Alvarezsauroidea, Therizinosauria and Paraves for lateral system? teeth venom
Possible functionality, adaptation or causes

Crown cutting along the whole crown height leading to deeper wounds

Slicing flesh on the crown width causing wide cuts and open wounds (predatory lifestyle?)

Trauma, aberrant tooth replacement or genetic factors

Causing wide cuts and open wounds, possibly gripping function (predatory lifestyle?)

Slicing flesh, gripping function, possibly removing feather/fur

Slicing through resistant structures such as fibrous material (herbivory/ omnivory)

Tooth simplification, precursor of unserrated crown

Denticle reduction due to tooth simplification

Trauma or genetic factor?

Piercing or slicing function?

Crown strength

Piercing, gripping and keeping slippery prey

Suction minimization, crown strength, or byproduct of growth

Suction minimization, crown strength, or byproduct of growth

Distributing stresses and/or preventing suction, possibly hosting septic bacteria or helping entrance of

Crown strength, venom delivery 
TABLE 3 (continued).

\begin{tabular}{|c|c|c|}
\hline Dental feature & Distribution & $\begin{array}{l}\text { Possible functionality, adaptation } \\
\text { or causes }\end{array}$ \\
\hline Longitudinal groove & Abelisauridae, Paraves & $\begin{array}{l}\text { Crown strength, venom delivery } \\
\text { system? }\end{array}$ \\
\hline Basal striations & Herrerasauridae, Proceratosaurus & Crown strength, byproduct of growth? \\
\hline $\begin{array}{l}\text { Irregular enamel } \\
\text { texture }\end{array}$ & $\begin{array}{l}\text { Herrerasauridae, Eoraptor, Liliensternus, Sinosaurus, Abelisauroidea } \\
\text { (Masiakasaurus excluded), Erectopus, Irritator, Allosaurus, } \\
\text { Metriacanthosauridae, Tyrannosaurinae and most of non- } \\
\text { dromaeosaurid Neocoelurosauria }\end{array}$ & Crown strength \\
\hline $\begin{array}{l}\text { Braided enamel } \\
\text { texture }\end{array}$ & $\begin{array}{l}\text { Non-abelisaurid Ceratosauria, non-spinosaurid Megalosauroidea, } \\
\text { Carcharodontosauria, Aorun, Segnosaurus and most non-averostran } \\
\text { Theropoda, non-neocoelurosaur Coelurosauria and Eudromaeosauria }\end{array}$ & Crown strength \\
\hline $\begin{array}{l}\text { Veined and } \\
\text { anastomosed enamel } \\
\text { texture }\end{array}$ & $\begin{array}{l}\text { Incisivosaurus for the premaxillary dentition; Spinosauridae for the } \\
\text { whole dentition }\end{array}$ & $\begin{array}{l}\text { Crown strength for particularly high } \\
\text { stresses }\end{array}$ \\
\hline $\begin{array}{l}\text { Procumbent } \\
\text { premaxillary teeth }\end{array}$ & $\begin{array}{l}\text { Daemonosaurus, Masiakasaurus, Chilesaurus, Similicaudipteryx, } \\
\text { Ornitholestes, Epidexipteryx, Archaeopteryx }\end{array}$ & $\begin{array}{l}\text { Procumbency: prehension of small to } \\
\text { medium-sized prey and/or vegetation } \\
\text { (possibly branch raking/stripping) }\end{array}$ \\
\hline $\begin{array}{l}\text { Procumbent } \\
\text { maxillary teeth }\end{array}$ & $\begin{array}{l}\text { Dracovenator, Coelophysoidea, Dilophosaurus, Masiakasaurus, } \\
\text { Spinosauridae, and Scipionyx for mesial maxillary teeth; } \\
\text { Similicaudipteryx, Epidexipteryx, Archaeopteryx and possibly } \\
\text { Chilesaurus and Nqwebasaurus for the whole maxillary dentition }\end{array}$ & \\
\hline $\begin{array}{l}\text { Procumbent dentary } \\
\text { teeth }\end{array}$ & $\begin{array}{l}\text { Herrerasaurus, Daemonosaurus, Daliansaurus, Megapnosaurus, } \\
\text { Masiakasaurus, Duriavenator, Spinosaurus, Ornitholestes, } \\
\text { Haplocheirus, Incisivosaurus, and some Tyrannosauroidea and } \\
\text { Dromaeosauridae for mesial dentary teeth; Chilesaurus, } \\
\text { Shenzhousaurus, Similicaudipteryx and Epidexipteryx for the whole } \\
\text { dentary dentition }\end{array}$ & \\
\hline $\begin{array}{l}\text { Laterocumbent and } \\
\text { retrocumbent teeth }\end{array}$ & $\begin{array}{l}\text { Spinosaurinae (laterocumbent teeth); Eoraptor, some Coelophysoidea } \\
\text { and basalmost Neotheropoda, and juvenile Limusaurus (retrocumbent } \\
\text { premaxillary teeth); Panguraptor, Sciurumimus and several } \\
\text { Dromaeosauridae (retrocumbent maxillary teeth) }\end{array}$ & $\begin{array}{l}\text { Hold and keep struggling and/or } \\
\text { slippery prey, or keep an immobile } \\
\text { bite }\end{array}$ \\
\hline $\begin{array}{l}\text { Partial/complete } \\
\text { premaxillary } \\
\text { edentulism }\end{array}$ & $\begin{array}{l}\text { Partial: Limusaurus (juvenile), Caudipteryx; Complete: } \\
\text { Therizinosauroidea, Caenagnathoidea, and Ornithomimosauria more } \\
\text { derived than Pelecanimimus }\end{array}$ & $\begin{array}{l}\text { Edentulism: paralleled with the } \\
\text { development of a rhamphotheca and } \\
\text { gastric mill, weight saving demands, } \\
\text { abandon of carnivory (herbivory or } \\
\text { omnivory) }\end{array}$ \\
\hline $\begin{array}{l}\text { Partial/complete } \\
\text { maxillary edentulism }\end{array}$ & $\begin{array}{l}\text { Partial/anteriormost portion of the maxilla: Limusaurus (juvenile) and } \\
\text { Erlikosaurus; Partial/posterior portion of the maxilla: Limusaurus } \\
\text { (juvenile), basalmost ornithomimosaurs (i.e., Nqwebasaurus, } \\
\text { Pelecanimimus), Shuvuuia, Erlikosaurus, Similicaudipteryx, many } \\
\text { troodontids, and Epidexipteryx; Complete: Caudipteryx }+ \\
\text { Caenagnathoidea, and Ornithomimosauria more derived than } \\
\text { Pelecanimimus }\end{array}$ & \\
\hline $\begin{array}{l}\text { Partial/complete } \\
\text { dentary edentulism }\end{array}$ & $\begin{array}{l}\text { Partial/anteriormost portion of the dentary: Limusaurus (juvenile), } \\
\text { Therizinosauroidea, and non-caenagnathoid Oviraptorosauria; Partial/ } \\
\text { posterior portion of the dentary: Limusaurus (juvenile), non- } \\
\text { ornithomimoid ornithomimosaurs more derived than Pelecanimimus, } \\
\text { Similicaudipteryx, and possibly Protarchaeopteryx and all } \\
\text { Scansoriopterygidae; Complete: Caudipteryx + Caenagnathoidea, } \\
\text { Ornithomimoidea }\end{array}$ & \\
\hline Complete edentulism & $\begin{array}{l}\text { All theropods more derived than Eodromaeus for the pterygoid; } \\
\text { Limusaurus, Ornithomimoidea and Caenagnathoidea for the whole } \\
\text { skull }\end{array}$ & \\
\hline
\end{tabular}


TABLE 4. Most conspicuous dental features in 31 theropod clades. Dental features for important subclades and taxa are also provided in brackets. 'small crowns in comparison to the upper and lower jaws' height.

\begin{tabular}{|c|c|}
\hline Clade & Dental features \\
\hline Non-neotheropod Theropoda & $\begin{array}{l}\text { Small to medium-sized ziphodont dentition; subcircular cross-section of mesial crowns; } \\
\text { mesial carina of mesialmost crowns, when present, facing labially and not reaching the } \\
\text { cervix; dentary teeth weakly to strongly procumbent; mesial carina, when present, straight } \\
\text { and reaching the cervix in some lateral teeth; mesialmost basal striations on the crown } \\
\text { (Herrerasaurus); procumbent premaxillary teeth (Daemonosaurus); fluted mesial and lateral } \\
\text { teeth (Tawa) }\end{array}$ \\
\hline Coelophysoidea & $\begin{array}{l}\text { Mesialmost teeth unserrated or with unserrated mesial carina; mesial dentition and mesial } \\
\text { maxillary dentition with slender, elongated and strongly recurved (i.e., strongly concave distal } \\
\text { profile) teeth; diastema between premaxillary and maxillary teeth; constricted tooth-row } \\
\text { between premaxilla and maxilla; retrocumbent premaxillary teeth; procumbent mesial } \\
\text { maxillary teeth; procumbent mesial dentary teeth (Megapnosaurus and some Coelophysis } \\
\text { specimens); fluted mesial and lateral teeth (juvenile Coelophysis) }\end{array}$ \\
\hline
\end{tabular}

Non-averostran Neotheropoda Mesial dentary teeth significantly larger than mid- and posterior dentary teeth; terminal (Liliensternus, Zupaysaurus, rosette of dentary with four to five teeth; mesial carina of mesial teeth denticulated, facing Sinosaurus, Dracovenator, and labially and extending basally well-above the cervix; mesial carina of mesial teeth centrally Dilophosaurus) positioned or slightly labially deflected from the mesial margin of the crown; retrocumbent premaxillary teeth; procumbent mesial maxillary teeth; mesial carina of mesial teeth reaching the cervix in some lateral teeth; DSDI>1.2 (Dracovenator and Dilophosaurus)

Non-abelisauroid Ceratosauria Mesial carina of mesial teeth restricted to the apical portion of the crown and facing labially in (Berberosaurus, Saltriovenator, mesialmost teeth; lateral dentition with a few strongly labiolingually compressed crown and Ceratosauridae) $(\mathrm{CBR}<0.4)$; mid-maxillary teeth taller than the dentary height; mesial and lateral crowns with a strongly labially deflected distal carina; labial surface of the crown weakly convex, almost flat, in some lateral teeth; mesial carina of lateral teeth extends to the cervix or slightly above it; concave surface(s) on the lingual and/or labial sides and adjacent to the mesial and/or distal carinae in some crowns; mesial teeth with fluted lingual surfaces (Ceratosaurus)

Abelisauridae Mesial carina reaching the cervix in mesial and lateral teeth; distal profile of crown weakly concave, straight or convex; lateral teeth with mesial and distal carinae on the mid-line of the mesial and distal profile, respectively; irregular enamel surface texture; subrectangular premaxillary and maxillary alveoli; Salinon to J-shaped cross-sectional outline of mesial teeth in most taxa; apically hooked distal denticles in some taxa

Noasauridae

Crown height lower than $2 \mathrm{~cm}$; irregular enamel surface texture; mesial carina of lateral teeth reaching the cervix, DSDI>1.2 (Noasaurinae); procumbent mesial dentition, mesial dentary teeth basally constricted, fluted and with a salinon-shaped cross-sectional outline (Masiakasaurus, possibly in Noasaurinae); flutes, hooked distal denticles and constriction between crown and root in some lateral teeth (Masiakasaurus); unserrated teeth and partial edentulism in juveniles, complete edentulism in adults (Limusaurus, possibly in Elaphrosaurinae)

Piatnitzkysauridae

Mesial teeth mesiodistally short and elongated, with a lenticular cross-sectional outline at the cervix; mesialmost teeth with a mesial carina facing labially; DSDI>1.2; mesial carina extending above the cervix in mesial and most lateral teeth; distal carina strongly deflected labially, enamel undulations and short to well-developed interdenticular sulci between distal denticles in some teeth; braided enamel texture; hooked distal denticles and mesial carina spiraling mesiolingually and reaching the root in some lateral crowns (Piatnitzkysaurus)

Megalosauridae

Mesial teeth mesiodistally short and elongated, with a lenticular cross-sectional outline at the cervix; mesialmost teeth with a mesial carina facing labially; mesial carina extending wellabove the cervix in mesial and lateral teeth; short to well-developed interdenticular sulci in between distal denticles in some teeth; braided enamel texture; transverse/marginal undulations in some teeth in most taxa; bilobate mesial denticles and well-visible transverse undulations in some lateral crowns (Megalosaurinae)

Spinosauridae

Conidont dentition; strong allometric heterodonty along the premaxillary, maxillary and dentary dentitions, with the largest crowns bore mesially; more than 5 premaxillary teeth; procumbent mesial maxillary teeth; terminal rosette of dentary with four to five teeth; mesial carina reaching the cervix, and extending on part of the root, in most/all teeth; fluted mesial and lateral teeth; veined/anastomosed enamel surface texture; enamel curving basally adjacent to the carinae; large number of minute denticles changing sporadically in size along the carinae (Baryonychinae); unserrated and beaded carinae, laterocumbent teeth, and distal profile of crowns weakly concave, straight or convex (Spinosaurinae) 
TABLE 4 (continued).

\begin{tabular}{|c|c|}
\hline Clade & Dental features \\
\hline Metriacanthosauridae & $\begin{array}{l}\text { Mesial and distal crowns with denticulated mesial and distal carinae extending to the root; D- } \\
\text { to salinon-shaped cross-sectional outline at the crown-base in mesialmost teeth; mesial } \\
\text { crowns with mesial carinae spiraling mesiolingually and lingually positioned longitudinal } \\
\text { groove adjacent to the mesial carina; lateral teeth with flat to concave labial depressions at } \\
\text { the crown-base (8-shaped cross-sectional outline of some lateral teeth); short to well- } \\
\text { developed interdenticular sulci between distal denticles; irregular enamel surface texture }\end{array}$ \\
\hline Allosauridae & $\begin{array}{l}\text { Pachydont dentition; mesial and distal crowns with denticulated mesial and distal carinae } \\
\text { extending to the root, and well-beneath the cervix in some teeth; mesial crowns with lingually } \\
\text { positioned longitudinal groove adjacent to the mesial carina; D- to J-and salinon-shaped } \\
\text { cross-sectional outline at the crown-base in mesialmost teeth; mesial teeth and mesial } \\
\text { maxillary crowns with mesial carinae spiraling mesiolingually; mesial and most lateral teeth } \\
\text { with strongly labially deflected distal carina; marginal and transverse undulations well-visible } \\
\text { on some crowns; short to well-developed interdenticular sulci in lateral teeth; dentition with a } \\
\text { combination of irregular and braided enamel surface texture }\end{array}$ \\
\hline
\end{tabular}

Neovenatoridae (with Megaraptora)

Carcharodontosauridae

Non-tyrannosaurid Tyrannosauroidea

Tyrannosauridae

Compsognathidae

Ornithomimosauria

Alvarezsauroidea (Aorun, Haplocheirus, and Alvarezsauridae)
$\mathrm{J}$-shaped cross-section of mesial teeth with concave surface adjacent to mesial carina on the lingual surface of the crown; concave surfaces on the labial/lingual surfaces and adjacent to the distal carina in some lateral crowns; braided enamel surface texture; mesial carina absent and figure-8-shaped cross-section of lateral crowns (Megaraptor and Orkoraptor)

Subcircular cross-section in mesialmost teeth; mesial carina of mesial teeth facing labially and not reaching the cervix; mesialmost teeth with strongly labially displaced distal carina; lateral crowns higher than $6 \mathrm{~cm}$; $\mathrm{DSDI}<0.9$ in lateral teeth; mesial carina reaching the cervix in some/most lateral crowns; well-visible marginal and/or transverse undulations in some lateral teeth; short to well-developed interdenticular sulci in lateral teeth; braided enamel surface texture; bilobate mesial denticles in many taxa; weakly sigmoid distal profile with basal half concave and apical half convex (Carcharodontosauridae more derived than Acrocanthosaurus); weak constriction between crown and root in some lateral teeth (Carcharodontosaurinae); pronounced marginal undulations adjacent to mesial and distal carinae in lateral teeth (Carcharodontosaurus saharicus)

Mesial teeth significantly smaller than lateral teeth; J- to U-shaped cross-sectional outline of mesial teeth; concave surface adjacent to mesial carina in mesial teeth; DSDI>1.2 in most taxa; some lateral crowns with a strongly labially deflected distal carina and a mesial carina extending above the cervix; interdenticular sulci between distal denticles in some lateral crowns; braided enamel surface texture; basal striations in mesial teeth (Proceratosaurus)

Pachydont dentition; mesial teeth with U-shaped cross-sectional outline and longitudinal ridge centrally positioned on lingual surface; $C B R>1.2$ in mesial teeth; transitional dentition with spiraling mesial carina and strongly labially deflected distal carina; short to welldeveloped interdenticular sulci in some teeth; irregular enamel texture and teeth with split mesial carinae in some taxa

Ziphodont dentition; teeth lower than $1 \mathrm{~cm}$; conidont mesial/mesialmost teeth; distal denticulated carina not reaching the cervix and/or the apex in some lateral teeth; unserrated mesial dentition and lateral dentition with unserrated mesial carina in most taxa; retrocumbent premaxillary teeth (Juravenator); mesial denticulated carina (Sinocalliopteryx)

Unserrated crowns; small crowns*, with crown height lower than $1 \mathrm{~cm}$; conidont dentition made of subsymmetrical isodont crowns (all ornithomimosaurs but Pelecanimimus); posterior portions of maxilla and dentary edentulous (non-ornithomimoid ornithomimosaurs); premaxilla and maxilla fully edentulous (ornithomimosaurs more derived than Pelecanimimus); complete edentulism (Ornithomimoidea); D-shaped mesialmost teeth, constricted mesial and lateral teeth (Pelecanimimus)

Crown height lower than $1 \mathrm{~cm}$; small crowns*; unserrated mesial teeth; closely packed mesial and lateral dentition with subsymmetrical (i.e., mesial and distal profiles convex), unserrated and weakly constricted isodont crowns (Alvarezsauridae/Parvicursorinae); faint longitudinal grooves on the apex (Mononykus) 
TABLE 4 (continued).

\begin{tabular}{|c|c|}
\hline Clade & Dental features \\
\hline Therizinosauria & $\begin{array}{l}\text { Folidont dentition made of subsymmetrical isodont crowns with convex mesial and distal } \\
\text { profiles; crown height lower than } 1 \mathrm{~cm} \text {; small crowns*; mesial and distal serrated carinae } \\
\text { never reaching the cervix; partially edentulous dentary; edentulous premaxilla, mesial and } \\
\text { distal serrated carinae extending above the cervix, small number of large typically apically } \\
\text { oriented mesial and distal denticles (Therizinosauroidea); Incisiform mesial teeth with a D- } \\
\text { shaped cross section, minute denticles, twisted mesial carina, transverse undulations, and } \\
\text { longitudinal ridges in some/all lateral teeth (Falcarius); split distal carina, sporadic variation of } \\
\text { denticles size, conidont distalmost dentary dentition (Segnosaurus) }\end{array}$ \\
\hline
\end{tabular}

Oviraptorosauria

Halszkaraptorinae and Unenlagiinae

Microraptorinae

Dromaeosaurinae

Velociraptorinae

Anchiornithinae

Jinfengopteryginae
Conidont mesial dentition; strong allometric heterodonty, with mesial teeth significantly larger than distal teeth; unserrated mesial crowns; lateral dentition with crown height lower than 1 $\mathrm{cm}$; folidont lateral dentition (Incisivosaurus and Similicaudipteryx); anastomosed enamel surface texture in mesial teeth (Incisivosaurus); partial premaxillary edentulism and complete maxillary and dentary edentulism (Caudipteryx); procumbent maxillary and dentary teeth (Similicaudipteryx); complete edentulism (Caenagnathoidea)

Large number of small crowns*; unserrated and strongly distally recurved crowns; irregular and non-oriented enamel surface texture; ridged/fluted lateral teeth (Unenlagiinae); ziphodont lateral crowns (Halszkaraptorinae and Unenlagiinae other than Austroraptor); weakly labiolingually compressed lateral teeth (Halszkaraptorinae and Unenlagiinae other than Buitreraptor); more than five closely packed premaxillary teeth and D-shaped mesial teeth (Halszkaraptor/possibly Halszkaraptorinae); conidont dentition, mesial dentary teeth significantly larger than distal ones (Austroraptor); strongly labiolingually compressed lateral teeth with 8-shaped cross-sectional outline at the crown-base (Buitreraptor)

Ziphodont dentition whose mesial and lateral dentitions include some unserrated crowns; some mesial teeth with a concave surface on the lingual surface and adjacent to the mesial carina; mesial carina not reaching the cervix; some lateral crowns with unserrated mesial carina and fluted/ridged labial surface; labial depression on the crown-base in some lateral teeth; DSDI>1.2 in lateral teeth (all microraptorines other than Microraptor); some constricted crowns in the mesial and lateral dentitions, unserrated mesial carina, and small number of distal denticles in some lateral crowns (Microraptor)

Ziphodont teeth with serrated mesial and distal carinae in all lateral teeth; J-shaped mesial teeth; mesial dentition with serrated mesial carina spiraling mesiolingually and extending close to the cervix; transverse undulations in some teeth; DSDI $>1.2$ in lateral teeth (all dromaeosaurines other than Dromaeosaurus); DSDI $\approx 0$ or $<0.9$, twisted mesial carina in lateral teeth (Dromaeosaurus); retrocumbent maxillary teeth, hooked denticles (Atrociraptor); procumbent dentary teeth (Utahraptor)

Ziphodont dentition with serrated mesial and distal carinae in at least some lateral teeth, DSDI > 1.2, mesial carina not reaching the cervix in most lateral teeth (all velociraptorines other than Tsaagan); some lateral teeth with a figure-of-eight-shaped cross-sectional outline of the crown-base (all velociraptorines other than Deinonychus); many taxa with ridged lateral crowns (e.g., Bambiraptor, Velociraptor, Linheraptor, Acheroraptor); some taxa with retrocumbent maxillary teeth (e.g., Bambiraptor, Deinonychus); mesial carina absent/ unserrated in all teeth (Tsaagan); hooked distal denticles (Saurornitholestes); mesialmost teeth with fluted labial surface (Velociraptor)

Folidont dentition with weakly to strongly distally recurved crowns; crown height lower than 1 $\mathrm{cm}$; mesial dentition unserrated; mesial dentary teeth closely packed; teeth from middle and distal portion of the lateral dentition sparsely spaced; small crowns* (anchiornithines other than Caihong); unserrated lateral teeth (anchiornithines other than Caihong and Liaoningvenator); mesial denticles and apically hooked denticles in some lateral teeth (Caihong)

Folidont dentition with fully unserrated teeth; mesial teeth closely packed; small crowns; dentary teeth resting in an open alveolar groove; posterior portion of maxilla edentulous; dentition with some unconstricted teeth (Jinfengopteryx) 
TABLE 4 (continued).

\begin{tabular}{ll}
\hline \multicolumn{1}{c}{ Clade } & \multicolumn{1}{c}{ Dental features } \\
\hline Troodontinae & $\begin{array}{l}\text { Folidont dentition with distally recurved crowns in the central and distal portions of the lateral } \\
\text { dentition; D- to salinon-shaped mesial dentition; mesial teeth closely packed; dentary teeth } \\
\text { resting in an open alveolar groove; fully unserrated dentition in many taxa (e.g., Mei, } \\
\text { Xixiasaurus, Urbacodon, Gobivenator, Byronosaurus); heterodont lateral dentition made of } \\
\text { folidont and ziphodont crowns in many basal forms (e.g., Xixiasaurus, Sinovenator, } \\
\text { Jianianhualong. Byronosaurus); some lateral teeth with a small number of large distal } \\
\text { denticles in derived forms (e.g., Sinornithoides, Troodon, Zanabazar, Saurornithoides); } \\
\text { mesial denticulated carina (Troodon, Pectinodon); interdenticular sulci and transverse } \\
\text { undulations in some teeth (Troodon) } \\
\text { Conidont dentition; unserrated teeth; strong allometric heterodonty, with mesial teeth } \\
\text { significantly larger than distal ones; procumbent premaxillary and dentary teeth; maxilla and } \\
\text { dentary partially edentulous }\end{array}$ \\
\hline Scansoriopterygidae &
\end{tabular}

2007; Shen et al., 2017a, 2017b; Xu et al., 2017; Pei et al., 2017b; Figure 10.2). If constricted teeth appear to be present in the majority, if not all troodontids, many of them (e.g., Byronosaurus, Daliansaurus, Sinovenator, Sinusonasus, Xixiasaurus) have a lateral dentition that also includes non-constricted ziphodont teeth. Constricted teeth are typically present in the anterior portion of the maxilla and dentary, where they are closely packed. In non-maniraptoriform theropods, constricted teeth in the whole dentition are only present in the noasaurid Masiakasaurus (UA 8680, 9091; FMNH P 2476) and the basal tetanuran Chilesaurus (Novas et al., 2015; see Baron and Barrett (2017) for a different opinion on the classification of Chilesaurus). Yet not all teeth are constricted in Masiakasaurus, and the lateral teeth only show a weak constriction between crown and root (Figure 10.1).

Mesial dentition. A weakly constricted crown at the level of the cervix can be seen in the premaxillary teeth of some abelisaurids such as Chenanisaurus (Longrich et al., 2017) and Majungasaurus (Rpm1 of FMNH PR 2008). A basal constriction between crown and root is also present in some premaxillary teeth of the basal tyrannosauroid Proceratosaurus (Rauhut et al., 2010; Figure 10.4), and the basal coelurosaur Ornitholestes (AMNH 619). Zinke and Rauhut (1994) mentioned the presence of a constriction in some mesial dentary teeth in the compsognathid Compsognathus. Although a weak constriction might be present in some premaxillary and lateral teeth, this feature is not clearly observable in the two Compsognathus specimens (MNHN CNJ 79; BSP AS I563). In dromaeosaurids, a week constriction is present in some premaxillary teeth of the basal dromaeosaurid Halszkaraptor (MPC-D 102-119). Zhou et al.
(2000) noted a constricted premaxillary crown in the basal oviraptorosaur Caudipteryx but a close examination of the teeth of specimen IVPP V12430 failed to confirm this observation. As other toothed oviraptorosaurs, the premaxillary dentition of Caudipteryx is conidont.

Lateral dentition. In the oviraptorosaurs Incisivosaurus (IVPP V13326; Osmólska et al., 2004), Protarchaeopteryx (Ji et al., 1998), and Similicaudipteryx (Li et al., 2018, figure S3f), as well as the therizinosaurs Erlikosaurus (Zanno et al., 2016) and Falcarius (Zanno, 2010a), only the lateral dentition is constricted between crown and root, the mesial dentition of these taxa being conidont or subconical/incisiform (Zanno, 2010a; Zanno and Makovicky, 2011). Likewise, the juvenile megalosaurid Sciurumimus (BMMS BK 11, Rdt4, Rdt6) and the carcharodontosaurids Carcharodontosaurus (SGM Din 1) and Giganotosaurus (MUCPV$\mathrm{CH}-1$ ) clearly show a ziphodont dentition but a few lateral teeth are weakly constricted at the cervix. Some lateral teeth of the microraptorine IVPP V13476 (Xu and Li, 2016) and most Microraptor specimens (e.g., IVPP V12330, V13320, V13475; BMNHC PH881; Xu et al., 2000; Turner et al., 2012; Pei et al., 2014) are also constricted. A constriction between crown and root is also visible in at least one lateral tooth of the putative dromaeosaurid Richardoestesia gilmorei (Rdt13, Currie et al., 1990 , figure $8.4 \mathrm{G}$ ). Only the distal teeth of the lateral dentition are constricted in the basal maniraptoriform Fukuivenator (Azuma et al., 2016), a dental synapomorphy of this taxon.

Remarks and synapomorphy. Constricted teeth are present in the mesial and/or lateral dentition of abelisauroids, carcharodontosaurines, proceratosaurids and the majority of non-avian maniraptoriforms. With some exceptions (Sciurumimus, 


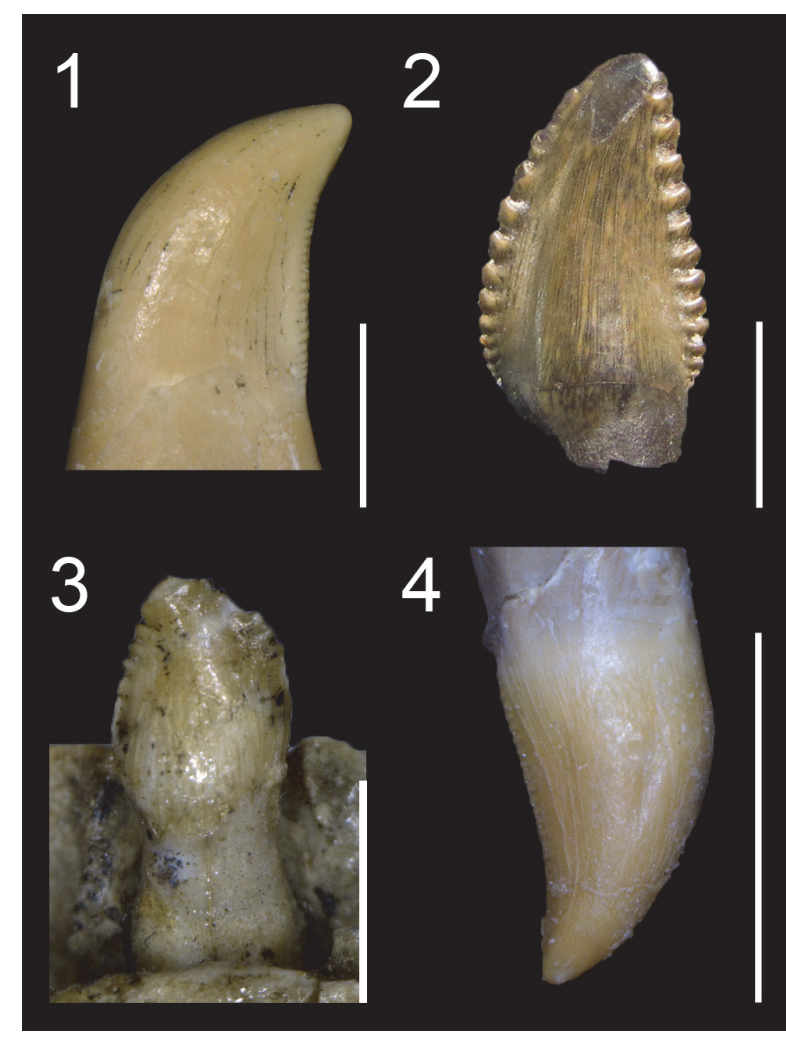

FIGURE 10. Basal constriction in non-avian Theropoda. 1, Isolated lateral tooth of the noasaurid Masiakasaurus knopfleri (FMNH PR 2476) in labial view; 2, Isolated tooth of the troodontid Troodon formosus (DMNH 22837) in labial view; 3, Thirteenth left dentary tooth of the therizinosaur Eshanosaurus deguchiianus (IVPP V11579) in lingual view; 4, Fourth right premaxillary tooth of the proceratosaurid Proceratosaurus bradleyi (NHMUK PV R.4860) in labial view. Scale bars equal 2 $\mathrm{mm}(1), 3 \mathrm{~mm}(3), 5 \mathrm{~mm}(2,4)$.

carcharodontosaurines, Chilesaurus, Halszkaraptor, Masiakasaurus, Microraptor, Ornitholestes, Pelecanimimus, Proceratosaurus and some abelisaurids), constricted teeth appear to be absent in all dromaeosaurids and all non-maniraptoriform theropods. It is unknown whether some lateral teeth of scansoriopterygids are weakly constricted as this condition cannot be positively confirmed in Epidexipteryx (IVPP V15471) and Yi (STM 31-2). The constriction occurs at approximately the same level on both mesial and distal sides of the teeth, and at the level of the cervix in all non-avian theropods displaying this feature (C.H. personal obs.). In some Archaeopteryx specimens, the tooth constriction is present slightly apical to the root in the lateral dentition, and well-above the cervix in mesial teeth (e.g., BSPG 1999 I 50; 11 th specimen of Archaeopteryx). Likewise, the constriction on the mesial margin occurs more apically than that on the distal margin in some lateral teeth of Archaeopteryx.

A constriction at the base of the crown appeared independently in noasaurids, abelisaurids, carcharodontosaurids and coelurosaurs throughout the evolution of theropods (Appendix 6.1 for mesial and lateral teeth). Despite the presence of a constriction in some mesial and possibly lateral teeth of Ornitholestes (AMNH 619), constricted teeth are not present in basal Ornithomimosauria (i.e., Nqwebasaurus) and Alvarezsauroidea (i.e., Aorun, Haplocheirus) implying that this feature is not synapomorphic for Maniraptora [sensu Senter (2011) and Turner et al. (2012)]. Pelecanimimus is the only known ornithomimosaur with folidont teeth suggesting that this feature may be autapomorphic for this taxon (e.g., Makovicky et al., 2010; Jin et al., 2012; Turner et al., 2012). Folidonty is synapomorphic for the clade Therizinosauria + Pennaraptora (Figure 8; Appendix 6.1 for lateral teeth). Constricted teeth were lost in the large majority of dromaeosaurids but appear to be retained, or re-acquired, in the mesial dentition in the basalmost member of Dromaeosauridae Halszkaraptor and in the lateral dentition of Microraptor. A folidont dentition appeared several times throughout the evolution of theropod dinosaurs, occurring in: Pelecanimimus (Pérez-Moreno et al., 1994); Alvarezsauridae/Parvicursorinae; and Therizinosauria + Pennaraptora.

Functional morphology. A marked constriction at the cervix, giving a lanceolate outline of the crown, is typically correlated with a diet involving a mixture of food, and primarily plant material (e.g., Galton, 1984, 1985; Barrett, 2000). Whilst the labiolingual compression of the tooth allows keeping a sharp edge that cuts food items, it is possible that a mesiodistal expansion of the crown relative to the root increases the surface area of the enamel as well as strengthening the crown by dissipating the forces applied apically along the tooth width. This hypothesis, however, requires to be tested with physical models or through FEA.

\section{Crown base ratio greater than 0.75}

A labiolingually broad crown is the typical condition of mesial teeth in non-avian theropods. A crown base ratio (CBR) greater than 0.75 is present in the mesial dentition of the majority of ziphodont theropods. However, a weak labiolingual compression of the whole crown (and not only the base) is also present in the dentition of Spinosauridae, which bear subconical teeth (e.g., Charig and 
Milner, 1997; Sereno et al., 1998; Sues et al., 2002), and large/mature Tyrannosauridae, which possess pachydont teeth (e.g., Holtz, 2003, 2008; C.H. personal obs.). These taxa have a crownbase ratio higher than this arbitrary value in many or most teeth of the mesial and lateral dentitions. The allosauroid Allosaurus and the basal maniraptoriform Ornitholestes have thick incrassate lateral teeth (CBR >0.75) along part of the dentary row. Teeth from the maxilla and the distalmost portion of the dentary (typically from the eighth dentary crown in both Allosaurus and Ornitholestes) are moderately (CBR around 0.5 to 0.7 ) to strongly (CBR of less than 0.5) labiolingually narrowed in these two taxa (C.H. personal obs.). In maniraptoriforms, the dentition of all ornithomimosaurs, therizinosaurs, alvarezsaurids and most likely Caudipteryx have a CBR higher than 0.75. The mesial and lateral dentitions of many troodontids, however (e.g., MPC-D 100-1128, Byronosaurus, Zanabazar), also have a subcircular cross-sectional outline at the crown base (C.H. personal obs.).

Remarks and synapomorphy. The subcircular outline of the crown is a synapomorphy of Spinosauridae (Sereno et al., 1998) and Maniraptoriformes (Appendix 6.2 for lateral teeth), being present in at least some crowns in the basal forms of: spinosaurids (Baryonyx, Charig and Milner, 1997; Ostafrikasaurus if a Spinosauridae; Buffetaut, 2011); ornithomimosaurs (Nqwebasaurus; Choiniere et al., 2012); alvarezsauroids (Aorun, Haplocheirus; Choiniere et al., 2014b); and therizinosaurs (Falcarius; personal obs.). Pachydont lateral teeth (CBR >0.6) are absent in the nontyrannosaurid tyrannosauroids Xiongguanlong ( $\mathrm{Li}$ et al., 2010), Guanlong (IVPP V14531), the possibly immature Dryptosaurus (Brusatte et al., 2011; R. Molnar, personal commun., 2017) and Raptorex (Sereno et al., 2009), a probable juvenile of Tarbosaurus (Fowler et al., 2011b). This feature is present in the basal tyrannosaurids Albertosaurus (Buckley et al., 2010; Reichel, 2010) and Gorgosaurus (AMNH 5458; USMN 12814), and the alioraminine Qianzhousaurus (Lü et al., 2014) so that pachydont teeth (and pachydonty) are a synapomorphy of Tyrannosauridae. It should be noted that some non-tyrannosaurid tyrannosauroids such as Dilong (IVPP 14242) and Proceratosaurus (Gerke and Wings, 2016) also bear pachydont lateral teeth while most teeth of the lateral dentition of the alioraminine Alioramus are not pachydont (Brusatte et al., 2012).

Functional morphology. The expansion of the crown labiolingually adds resistance and the ability to withstand bending loads applied from all directions (e.g., Therrien et al., 2005; Snively et al., 2006; Holtz, 2008; Reichel, 2010). Thick teeth in carnivorous theropods are adapted to resist contact with hard items such as bones and scales during prey capture and feeding. The mesial dentition was most likely subject to higher stress and loads than the lateral dentition during bites. In theropods, lateral crowns are as labiolingually wide as those of the mesial dentition in pachydont and conidont theropod teeth, used for bone-crushing or to impale and hold prey, respectively (e.g., Therrien et al., 2005; Holtz, 2008). Interestingly, teeth with a CBR greater than 0.75 are also present in many maniraptoriforms such as ornithomimosaurs, therizinosaurs and alvarezsauroids, in which the crosssection of the crown base is sub-circular (e.g., Clark et al., 1994; Xu et al., 2001; Ji et al., 2003; Barrett, 2009; Choiniere et al., 2012; C.H. personal obs.). Increase in resistance of higher stresses in different directions in the dentition of these omnivorous/herbivorous theropods likely results from the consumption of hard plant material whereas labiolingually compressed blade-shaped ziphodont teeth of carnivores are suitable to slice through softer flesh.

\section{Crown height higher than $60 \mathrm{~mm}$}

Absolute tooth size is a homoplastic feature that also varies allometrically and must be treated with caution for classification purposes. Nonetheless, this feature has proven to be useful to discriminate the teeth of different theropod taxa (Smith, 2005; Smith et al., 2005; Han et al., 2011). Theropods bearing crowns larger than $6 \mathrm{~cm}$ are only known in non-neocoelurosaur averostrans: Allosauroidea (e.g., Acrocanthosaurus, Allosaurus, Carcharodontosaurus, Giganotosaurus, Mapusaurus, Sinraptor), Ceratosaurus, Megalosauridae (e.g., Afrovenator, Megalosaurus, Spinosaurus, Torvosaurus, Wiehenvenator), and some derived Tyrannosauroidea (e.g., Albertosaurus, Bistahieversor, Tarbosaurus, Tyrannosaurus). Abelisauroids, baryonychines, neovenatorids, piatnitzkysaurids, proceratosaurids, and non-neocoelurosaur averostrans, including dromaeosaurids have shorter teeth (Young et al., 2019; C.H. personal obs.) with even the crowns of the largest dromaeosaurids such as Utahraptor being less than $5 \mathrm{~cm}$ in height (C.H. personal obs.). However, given the possible immaturity of Baryonyx and Suchomimus (Charig and Milner, 1997; Hendrickx et al., 2016) and because the longest crown measured in Suchomimus is $54 \mathrm{~mm}$, it is likely that 
some baryonychines had crowns higher than $6 \mathrm{~cm}$. Likewise, the largest crowns of the proceratosaurid Yutyrannus are slightly shorter than $60 \mathrm{~mm}(\mathrm{C} . \mathrm{H}$. personal obs.), and some proceratosaurids or basal tyrannosauroids could have borne lateral crowns exceeding $60 \mathrm{~mm}$.

Remarks and synapomorphy. In the discussion on the taxonomic affinities of a large-sized theropod tooth (IVPP V15310; $\mathrm{CH}=92 \mathrm{~mm}$; $\mathrm{AL}=102$ $\mathrm{mm}$ ) from the Late Jurassic Shishugou Formation of China, Xu and Clark (2008) cite Carcharodontosaurus, Tyrannosaurus and probably Giganotosaurus as the theropods with a crown height subequal or higher than $90 \mathrm{~mm}$. We confirm that some Giganotosaurus lateral teeth have a $\mathrm{CH}$ higher than $9 \mathrm{~cm}$ (e.g., MUCPV-CH-1 L1) and add to this list Torvosaurus (e.g., BYU-VP 725 12817, ML 1100), Acrocanthosaurus (NCSM 14345, Lmx4) and most likely Spinosaurus based on the size of the partially complete Lmx4 in MSNM V4047.

With a $\mathrm{CH}$ of $145.5 \mathrm{~mm}$, the largest known theropod crowns belong to the megalosaurid Torvosaurus gurneyi (SHN.401 and SHN.450; CBL of $48 \mathrm{~mm}$ ) from the Upper Jurassic of Portugal (Malafaia et al., 2017a, 2017b). Other taxa may have possessed larger crowns: the spinosaurid Spinosaurus (CBL of $51 \mathrm{~mm}$ in Lmx4 of MSNM V4047 preserving the crown base only); the tyrannosaurid Tyrannosaurus $(\mathrm{CH}$ of $139 \mathrm{~mm}$ in Rdt3 of FMNH PR2081, the highest crown measured for this taxon; C.H. personal obs.); large-bodied tetanurans from the Kimmeridgian-Tithonian Tendaguru beds of Tanzania $(\mathrm{CH}$ slightly more than $135 \mathrm{~mm}$; CBL of $54 \mathrm{~mm}$ in NHMUK PV R.6758) and the Berriasian-Valanginian Kirkwood Formation of South Africa (CBL >53 mm in MB R.2352; Galton and Molnar, 2012). A lateral dentition including crowns higher than $60 \mathrm{~mm}$ is here considered a synapomorphy of Ceratosauridae, Megalosaurinae more derived than Duriavenator, Allosauroidea, and a clade encompassing Tyrannosauridae + Bistahieversor or Appalachiosaurus (Appendix 6.3 for lateral teeth).

\section{Unserrated teeth}

Mesial dentition. In embryonic/juvenile individuals, unserrated teeth restricted to the mesial dentition have been recorded in Aorun (Choiniere et al., 2014a), Archaeornithoides (Elzanowski and Wellnhofer, 1993), Coelophysis (Colbert, 1989; Figure 11.1), Daspletosaurus (Currie, 2003), Juravenator (Chiappe and Göhlich, 2010), Scipionyx (Dal Sasso and Maganuco, 2011), Sciurumimus (Rauhut et al., 2012; Figure 11.2), Timurlengia
(Averianov and Sues, 2012), Torvosaurus (Araújo et al., 2013) and Troodon (Varricchio et al., 2002). Unserrated teeth are also present in the premaxillary teeth of the "Jordan Theropod" LACM 28471 (Molnar, 1978), considered to be a specimen of Nanotyrannus (Larson, 2013) or a juvenile individual of Tyrannosaurus (Carr and Williamson, 2004). In mature individuals, unserrated teeth are restricted to the mesial dentition of some coelophysoids such as Megapnosaurus rhodesiensis (Raath, 1977) and 'Syntarsus' kayentakatae (Rowe, 1989), and the basal pantyrannosaurian Xiongguanlong (Li et al., 2010; Figure 11.4) and an indeterminate tyrannosauroid from the Cenomanian of Utah (Zanno et al., 2019). Most compsognathids such as Compsognathus, Juravenator and Sinosauropteryx also show unserrated mesial crowns, but these taxa may not have been fully grown individuals (i.e., juveniles to subadults; Currie and Chen, 2001; Peyer, 2006; Chiappe and Göhlich, 2010). Non-denticulate mesial teeth have also been identified in: anchiornithines (e.g., Liaoningvenator, Caihong; Shen et al., 2017b; Hu et al., 2018); Falcarius (UMNH 15097, 15223); Haplocheirus (Choiniere et al., 2014b); microraptorines (e.g., Microraptor, Sinornithosaurus, Shanag; Xu et al., 2000; Xu and Wu, 2001; Turner et al., 2007), non-anchiornithine troodontids (e.g., Daliansaurus, Sinovenator, Sinusonasus, Sinornithoides; Currie and Dong, 2001b; Xu et al., 2002b; Xu and Wang, 2004; Shen et al., 2017a); and Ornitholestes (AMNH 619).

Whole dentition. Unserrated teeth are present in the whole dentition of: the noasaurid Limusaurus [Wang et al.'s (2017a) data matrix]; spinosaurines such as Irritator (Sues et al., 2002; Figure 11.3), Angaturama (Kellner and Campos, 1996), and Spinosaurus (Stromer, 1915); the neocoelurosaur Fukuivenator (Azuma et al., 2016); basal ornithomimosaurs such as Nqwebasaurus (Choiniere et al., 2012; Figure 11.6), Pelecanimimus (Pérez-Moreno et al., 1994), and Shenzhousaurus (Ji et al., 2003), and all alvarezsaurids such as Shuvuuia (Chiappe et al., 1998; Figure 11.7) and Mononykus (Perle et al., 1993). Unserrated crowns are also present in the whole dentition of the basal oviraptorosaur Caudipteryx (IVPP 12430; NGMC 974 A; Figure 11.8 ), and all halszkaraptorine and unenlagiine dromaeosaurids such as Halszkaraptor (Cau et al., 2017), Mahakala (Turner et al., 2011), Buitreraptor (Gianechini et al., 2011b; Figure 11.9) and Austroraptor (Novas et al., 2009). Many non-anchiornithine troodontid like Mei (Xu and Norell, 2004), Byronosaurus (Makovicky et al., 2003), Gobivena- 


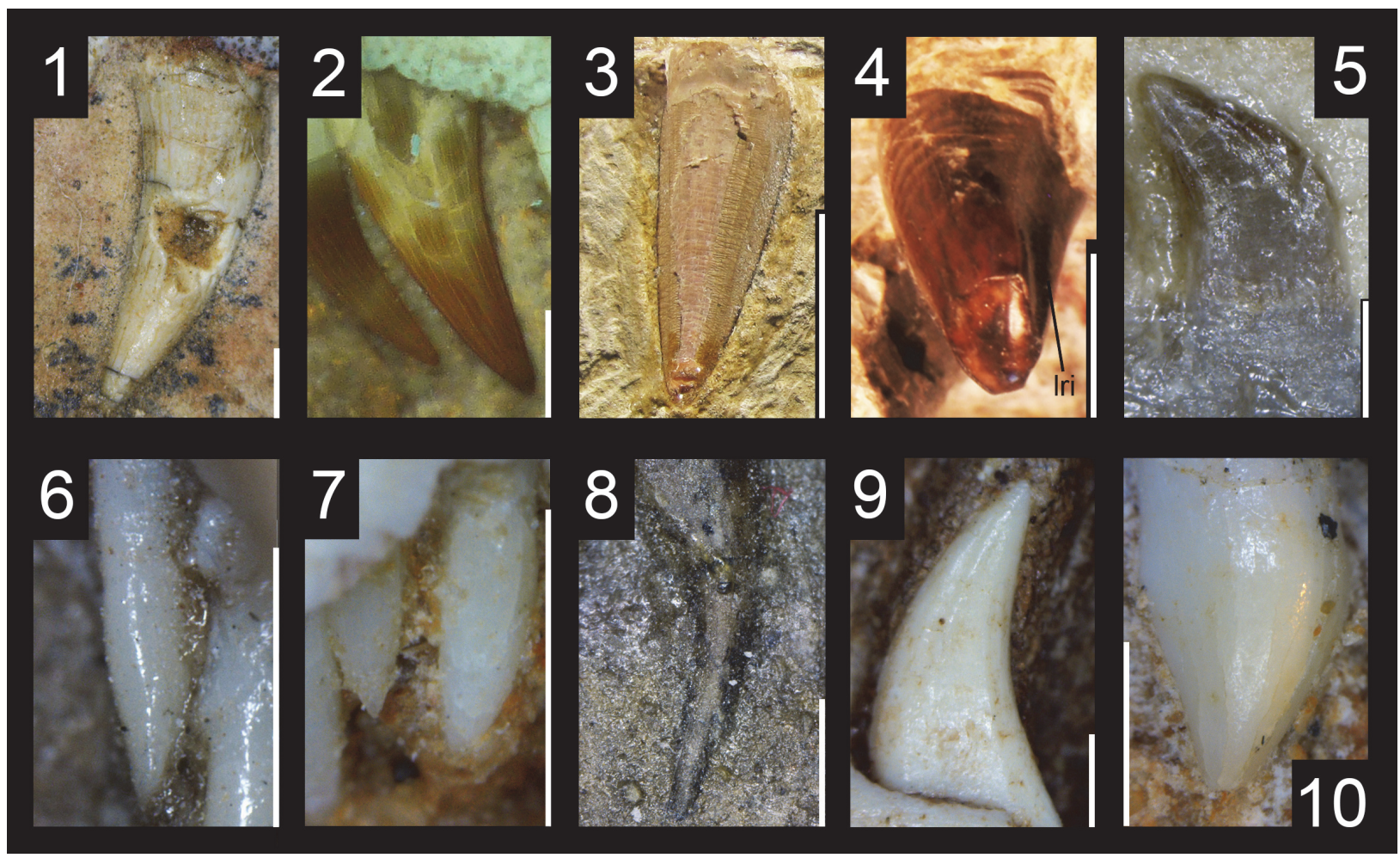

FIGURE 11. Unserrated teeth in non-avian Theropoda. 1, Second right premaxillary tooth of the coelophysoid Coelophysis bauri (DMNS 30596) in labial view; 2, First and second left premaxillary teeth of the megalosaurid Sciurumimus albersdoerferi (BMMS BK 11; courtesy of $\mathrm{H}$. Tischlinger and $\mathrm{O}$. Rauhut) in labial view; 3, Penultimate? right maxillary tooth of the spinosaurid Irritator challengeri (SMNS 58022) in labial view; 4, Third? right premaxillary tooth of the pantyrannosaurian Xiongguanlong baimoensis (FRDC-GS JB16-2-1; courtesy of P. Makovicky) in mesioapical view, showing the central ridge on the lingual surface of the crown; $\mathbf{5}$, Left dentary tooth of the compsognathid Compsognathus longipes (MNHN CNJ79) in lingual view; 6, Left maxillary tooth of the basal ornithomimosaur Nqwebasaurus thwazi (AM 6040) in labial view; 7, Right maxillary teeth of the alvarezsaurid Shuvuuia deserti (MPC-D 100-977) in labial view; 8, Second right? premaxillary tooth of the basal oviraptorosaur Caudipteryx zoui (IVPP V12430) in labial view; 9, Second left dentary tooth of the dromaeosaurid Buitreraptor gonzalezorum (MPCA 245) in labial view; 10, Right maxillary tooth of Almas ukhaa (MPC-D 100-1323) in labial view. Abbreviation: Iri, longitudinal ridge. Scale bars equal $1 \mathrm{~mm}(1-2,5-10), 5 \mathrm{~mm}(4), 1 \mathrm{~cm} \mathrm{(3).}$

tor (Tsuihiji et al., 2014), Urbacodon (Averianov and Sues, 2007), Xixiasaurus (Lü et al., 2010), IVPP V20378 and the jinfengopterygines Jinfengopteryx (Ji et al., 2005), Almas (Pei et al., 2017b; Figure 11.10) and MPC-D 100-1128, also have non-denticulate crowns all along their jaws. Unserrated teeth are also present in the whole dentition of the anchiornithines Anchiornis (IVPP V16055; Hu et al., 2009; Pei et al., 2017a), Eosinopteryx (YFGP-T5197; Godefroit et al., 2013b), Aurornis (YFGP-T5198; Godefroit et al., 2013a) and the basal avialan Archaeopteryx (e.g., Martin et al., 1980; Elzanowski and Wellnhofer, 1996; Mayr et al., 2007).

Remarks and synapomorphy. Protarchaeopteryx, interpreted as a basal oviraptorosaur and a senior synonym of Incisivosaurus (Senter et al., 2004), was described as possessing denticulated teeth (Ji et al., 1998). However, Senter et al. (2004) noted that no serrations could be observed in the holotype specimen of Protarchaeopteryx NGMC 2125. Nevertheless, minute denticles (60 denticles per $5 \mathrm{~mm}$ ) can be seen on the distal carina of Lmx6? in Incisivosaurus (IVPP V1326; contra Xu et al., 2002a) and a non-erupted dentary crown appears to have poorly delimited serrations on both mesial and distal carinae (C.H. personal obs.). Consequently, we follow Ji et al.'s (1998) description of Protarchaeopteryx and consider that serrations are likely to be present in NGMC 2125. The troodontid Zanabazar was described as having unserrated premaxillary teeth by Norell et al. (2009), yet the presence of denticles is clearly reported in the premaxillary teeth by Barsbold (1974) and at least distal serrations appear to be present in the mesial teeth of this taxon (Norell et 
al., 2009, figure 22). In the same way, although scored as having unserrated premaxillary teeth by Zhang et al. (2008, character 346), Epidexipteryx (IVPP V15471) appears to have an apicobasally short carina on the first right premaxillary tooth ( $r 2$ of Zhang et al., 2008, figure 1b) bearing minuscule serrations (C.H. personal obs.). All other teeth are unserrated and the presence of a denticulated carina on this tooth requires confirmation.

Besides Incisivosaurus and Epidexipteryx, a combination of distally denticulated crowns and unserrated teeth is present in the lateral dentition of: the compsognathids Compsognathus (MNHN CNJ 79; Stromer, 1934; Peyer, 2006) and Scipionyx (Dal Sasso and Maganuco, 2011); Ornitholestes (AMNH 619); the basal alvarezsauroids Aorun (IVPP V15709) and Haplocheirus (Choiniere et al., 2014b); the dromaeosaurid Shanag (Turner et al., 2007), Microraptor (IVPP V13330, V13475; Hwang et al., 2002), and IVPP V13476 (Xu and Li, 2016); and some anchiornithines and more derived troodontids such as Caihong (Hu et al., 2018), Liaoningvenator (Shen et al., 2017b), Jianianhualong (Xu et al., 2017), Sinovenator (IVPP V12615; Xu et al., 2002b) and Sinusonasus (IVPP V11527; Xu and Wang, 2004). Interestingly, these taxa typically have particularly small denticles (i.e., more than ten denticles per $\mathrm{mm}$ ), suggesting that taxa with unserrated teeth derive from forms bearing crowns with minute denticles. This is exemplified by three clades with unserrated teeth namely, Spinosaurinae, Alvarezsauridae, and Caudipteridae, which evolve from baryonychine, Haplocheirus and Incisivosaurus-like theropods, respectively, whose teeth are finely denticulated.

Unserrated lateral dentition is absent in mature individuals of Allosauroidea, Ceratosauria, Coelophysoidea, non-neotheropod Theropoda, non-spinosaurine Megalosauroidea, non-unenlagiine Dromaeosauridae, Therizinosauria, Tyrannosauroidea and most derived Troodontinae. The loss of denticles in lateral teeth is a trend that happened several times in the evolution of theropods. The teeth of Alvarezsauroidea, Ornithomimosauria, Oviraptorosauria and Spinosaurinae all lost denticles independently. With the presence of denticulated teeth in the basal members of Alvarezsauroidea (Haplocheirus), Therizinosauria (Falcarius) and Oviraptorosauria (Incisivosaurus), and the absence of denticles in basal taxa belonging to Avialae, Dromaeosauridae (Buitreraptor, Halszkaraptor), Troodontidae (e.g., Almas, Anchiornis, MPC-D 100-1128), and possibly Scansoriopterygidae, unserrated teeth is the apomorphic condition of the clade Paraves. Likewise, the presence of unserrated teeth is synapomorphic to Alvarezsauridae/Parvicursorinae (Appendix 6.5), Aves (sensu Choiniere et al., 2010b), Ornithomimosauria (Appendix 6.5), Caudipteridae + Caenagnathoidea, and Spinosaurinae (Appendix 6.7 for lateral teeth). On the other hand, denticles were independently reacquired by Anchiornithinae (e.g., Caihong, Liaoningvenator), derived Troodontinae (i.e., Sinovenator + more derived forms; Appendix 6.5, and 6.7 for mesial teeth) and the clade Microraptorinae + Eudromaeosauria (sensu Turner et al., 2012; Appendix 6.5 and 6.7 for mesial teeth). The total absence of serrations was thought to be a synapomorphy of the Unenlagiinae by Novas et al. (2009) and Gianechini et al. (2011b), yet their closest ancestors seem to share this feature as well, and unserrated teeth in Unenlagiinae is therefore considered to be a plesiomorphic condition (Appendix 6.5 and 6.7 for mesial teeth).

Functional morphology. The absence of serrations indicates less efficiency of slicing food (Xing et al., 2013b). Unserrated teeth are therefore used to either spear into flesh (Xing et al., 2013b) and deeply injure prey items, or for cropping and browsing vegetation. The absence of denticles seems also to result in the simplification of the crown in the theropod clades whose derived forms have lost their dentition (Gianechini et al., 2011b).

\section{Unserrated mesial carina, denticulated distal carina}

Mesial dentition. Mesial teeth with unserrated/ absent mesial carinae and denticulated distal carinae are present in most theropod clades. This feature is observed in: basal saurischians such as Eoraptor (PVSJ 512), Herrerasaurus (PVSJ 407) and Ischisaurus (PVSJ 605); the coelophysoid Coelophysis (CMNH 81765, 82931); the noasaurid Masiakasaurus (FMNH PR.2696); the megaraptoran Megaraptor (Porfiri et al., 2014); the basal pantyrannosaurians Dilong (IVPP V14242) and Aviatyrannis (MG 27801 D172); some compsognathids such as Juravenator (Chiappe and Göhlich, 2010) and Sinocalliopteryx (Ji et al., 2007); the therizinosaur Erlikosaurus (MPC-D 100-111; n.b. although the anteriormost portion of the dentary is edentulous, we consider the distally denticulated first and second dentary teeth to be from the mesial dentition); the dromaeosaurid Tsaagan (Norell et al., 2006); and the troodontids Linhevenator (Xu et al., 2011a; the mesial carina is the unserrated one in Linhevenator; contra Xu et al., 2011a) and possibly Saurornithoides (AMNH 
6516). Large distal denticles can indeed be seen in the latter, but the mesial teeth are too badly preserved to rule out the presence of mesial serrations. The ceratosaurid Ceratosaurus has a denticulated mesial carina in the premaxillary and dentary teeth (UMNH VP 5278; contra Currie and Carpenter, 2000; Bakker and Bir, 2004).

Lateral dentition. In lateral teeth, this condition is present in: the noasaurid Masiakasaurus (FMNH PR 2476), the juvenile megalosaurid Sciurumimus (Rauhut et al., 2012); some megaraptorans such as Orkoraptor (Novas et al., 2008) and Megaraptor (Porfiri et al., 2014); and all compsognathids, with Sinocalliopteryx and possibly Juravenator (a single tooth, Rmx8, shows what could be mesial denticles on the crown apex) having also a few lateral teeth with denticles on both carinae (e.g., JME Sch 200; Currie and Chen, 2001; Hwang et al., 2004; Peyer, 2006; Ji et al., 2007; Dal Sasso and Maganuco, 2011). A mesial carina is also absent in the lateral dentition of: the basal neocoelurosaur Ornitholestes (AMNH 619); the basal Alvarezsauroidea Aorun (Choiniere et al., 2014a) and Haplocheirus (Choiniere et al., 2014b); and the basal Oviraptorosauria Incisivosaurus. In paravians, a few dromaeosaurids such as Linheraptor (IVPP V16923), Microraptor (Xu et al., 2000; Hwang et al., 2002), Saurornitholestes (Currie et al., 1990), Shanag (Turner et al., 2007), Tsaagan (Norell et al., 2006; Xu et al., 2010) and Velociraptor (Godefroit et al., 2008) also show this condition. In Velociraptorinae, most teeth of Linheraptor and Velociraptor and all the dentition of Tsaagan lack a mesial carina. This dental feature is also seen in some anchiornithines like Caihong (Hu et al., 2018) and Liaoningvenator (Shen et al., 2017b), where distally denticulated teeth appear in the central and/or distal portion of the maxilla and dentary, and many non-anchiornithine troodontids including Daliansaurus (Shen et al., 2017a), Pectinodon (Carpenter, 1982; Larson and Currie, 2013), Saurornithoides (Norell et al., 2009), Sinornithoides (IVPP V9612; Currie and Dong, 2001b), Sinovenator (IVPP V12615), Sinusonasus (IVPP V11527), Troodon (Currie, 1987) and Zanabazar (Norell et al., 2009). Minute mesial denticles restricted to the apical part of the crown have been observed in at least one maxillary tooth of the basal alvarezsauroid Haplocheirus (IVPP V14988; contra Choiniere et al., 2014b).

Remarks and synapomorphy. Similar to the loss of serrations for the whole dentition, the loss of mesial denticles happened several times convergently in the evolution of theropods. The presence of lateral crowns with unserrated mesial carina and denticulated distal carina seems to be the derived condition in Megaraptora (sensu Benson et al., 2010) among Neovenatoridae, and neocoelurosaurs among Coelurosauria (Appendix 6.4 for lateral teeth). The reacquisition of denticles in the mesial carina also occurred independently in some anchiornithines like Caihong ( $\mathrm{Hu}$ et al., 2018), derived troodontids like Troodon and Pectinodon, and in members of the clade Microraptorinae + Eudromaeosauria.

\section{Concave surfaces adjacent to carinae on lateral teeth.}

A slightly concave or planar surface adjacent to the distal and mesial carinae in lateral teeth was considered to be a ceratosaurian (neoceratosaurian sensu Rauhut, 2004) synapomorphy by Rauhut (2004), however, this feature is widespread among non-coelurosaur theropods. Among nonaverostran theropods, a concave surface adjacent to the distal carina is visible on the labial (and in some cases lingual) side of some lateral crowns in the basal saurischian Eodromaeus (PVSJ 560, 561), the non-averostran neotheropod Dilophosaurus (UCMP 37303; Figure 12.1) and Coelophysis (CMNH 81765). As noted by Rauhut (2004), this concave surface is present in some Ceratosauridae such as Ceratosaurus (USNM 4735; UMNH VP 5278) and Genyodectes (MLP 26-39; Rauhut, 2004), but we could not identify this dental feature in the lateral teeth of any Abelisauridae or Noasauridae. A planar surface, however, is observable on the labial surface and adjacent to the distal carina in one lateral tooth of Skorpiovenator (MMCN-PV 48).

A concave area adjacent to the distal carina, on the labiobasal part of the crown, is present in the megalosauroid Piatnitzkysaurus (MACN CH 895) and Afrovenator (MNN TIG1; Figure 12.2). This concave surface is also present on one or both labial and lingual sides all over the crown in the metriacanthosaurid Sinraptor (IVPP 10600; Figure 12.4), the neovenatorid Neovenator (MIWG 6348; Figure 12.3), and some megaraptorans such as Fukuiraptor (Azuma and Currie, 2000, figure 4; Molnar et al., 2009, figure 3E) and Australovenator (Hocknull et al., 2009, figures 20K, 21E). This dental character would, therefore, support the clade Neovenatoridae recovered by Benson et al. (2010) and Carrano et al. (2012), as tyrannosauroids do not seem to display this feature (C.H. personal obs.). The lateral teeth of some troodontids such as Sinornithoides (IVPP V9612), Byronosaurus (MPC-D 100-983) and Urbacodon (Averianov and 


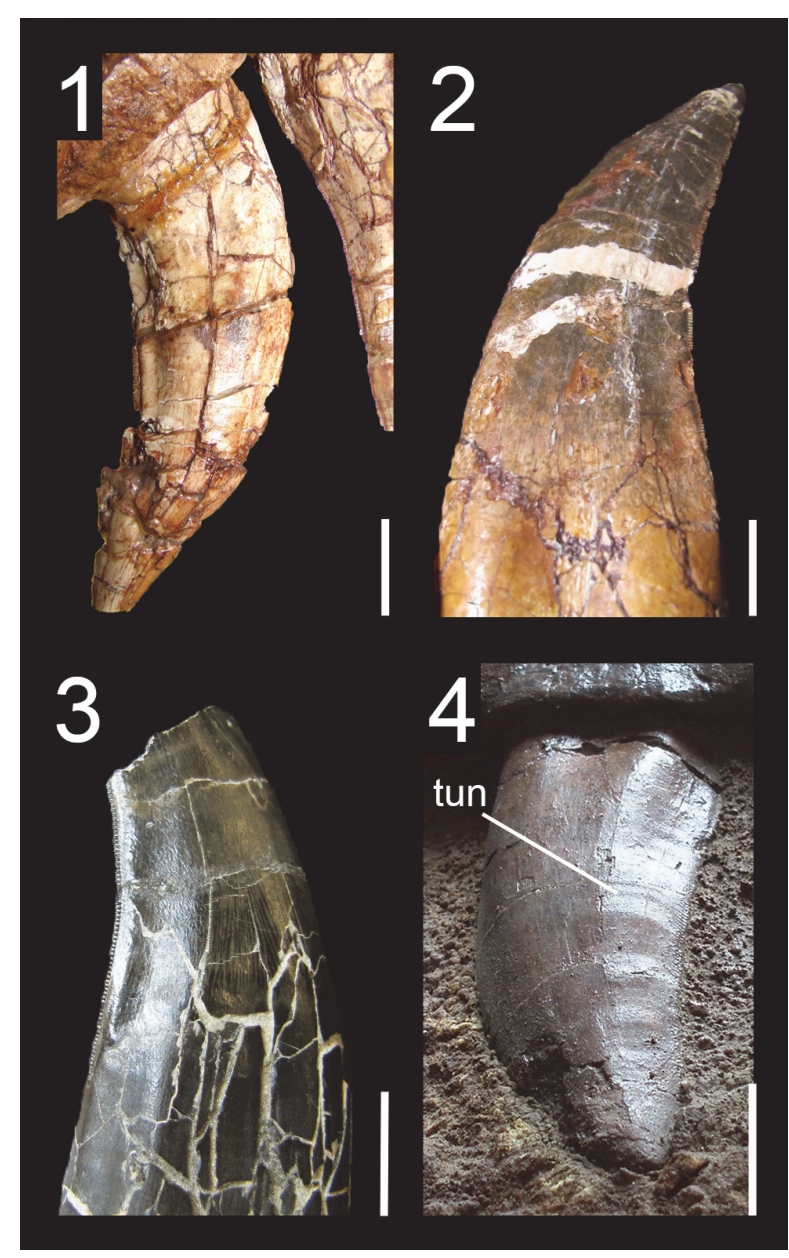

FIGURE 12. Concave surface adjacent to a carina in non-avian Theropoda. 1, Third left maxillary tooth of the dilophosaurid Dilophosaurus wetherilli (UCMP 37303) in lingual view; 2, Isolated tooth of the megalosaurid Afrovenator abakensis (MNN UBA1) in labial view; 3, Isolated tooth of the neovenatorid Neovenator salerii (MIWG 6348) in labial view; 4, Fifth left maxillary tooth of the metriacanthosaurid Sinraptor dongi (IVPP 10600) in labial view, also showing transverse undulations (courtesy of R. Benson). Abbreviation: tun, transverse undulation. Scale bars equal $1 \mathrm{~cm}$.

Sues, 2007) also show a concave surface adjacent to the distal carina on the labial surface of the crown. Some mesial and/or lateral teeth of the basal therizinosaurs Falcarius (UMNH VP 14545) and Jianchangosaurus (Pu et al., 2013, figure 4B) show a deep concave surface adjacent to the distal carina on the lingual surface.

Remarks and synapomorphy. Eoraptor lateral teeth (PVSJ 512) are unique in having a strongly mesiodistally convex surface labiomesially situated (the 'rounded eminence' of Sereno et al., 2013) and adjacent to a mesiodistally concave surface marginal to the distal carina. A similar concave surface is also present in the vicinity of the mesial carina in some lateral teeth of this taxon. A concave or planar surface adjacent to carinae in some lateral teeth is considered to be synapomorphic for Neovenatoridae (Figure 3.20; Appendix 6.8 for lateral teeth) and possibly for Ceratosauridae and Metriacanthosauridae.

Functional morphology. The presence of concave surfaces marginal to carinae or mesially-situated on the tooth had several functional implications in theropods, namely to either enhance the structural strength and stability of the crown by increasing the surface area of the enamel (Folinsbee et al., 2007), or to allow rapid penetration and easier withdrawal during the bite (Freedman, 1957).

\section{J-shaped and salinon-shaped cross-section}

Salinon-shaped cross-section. A concave surface marginal to both carinae and separated by a wide convexity on the lingual surface of the crown, therefore creating a salinon-shaped outline (sensu Hendrickx et al., 2015c; Figure 1.11) of the crown base in cross-section, is seen in the first mesial teeth (pm1-2, dt1) of abelisaurids such as Abelisaurus (MPCA 1, 5), Indosuchus (AMNH 1753) and Majungasaurus (Fanti and Therrien, 2007, figure 6C3; FMNH PR.2100; Figure 13.1). A concave surface adjacent to both carinae has also been observed in some mesial teeth of the noasaurids Masiakasaurus (e.g., UA 9128, FMNH PR.2182), the allosauroids Allosaurus (AMNH 600, 851; CMNH 21703; UMNH VP 2151, 6145, 7438) and Sinraptor (IVPP V10600), as well as the troodontids Troodon (Currie, 1987; Currie et al., 1990), Urbacodon (Averianov and Sues, 2007), and an indeterminate taxon from Uzbekistan (Averianov and Sues, 2007). A mesiodistally biconcave profile of the lingual surface of the crown is also present in the lateral teeth of the troodontid Xixiasaurus (the "distinct grooves adjacent to the carinae" of Lü et al., 2010, p. 384, figure 3A1).

J-shaped cross-section. A concave surface adjacent to the mesial carina only occurs on the lingual surface of more distal mesial teeth (pm3-4, mx1-2, dt2-3) of abelisaurids, which, therefore, have a Jshaped cross-sectional outline (Figure 1.12,13) of the crown base. A J-shaped cross-sectional outline here refers to labiolingually wide (Figure 1.12) or narrow crowns (Figure 1.13) characterized by a spiraling mesial carina facing mesiolingually or lingually, a distally oriented distal carina and the pres- 


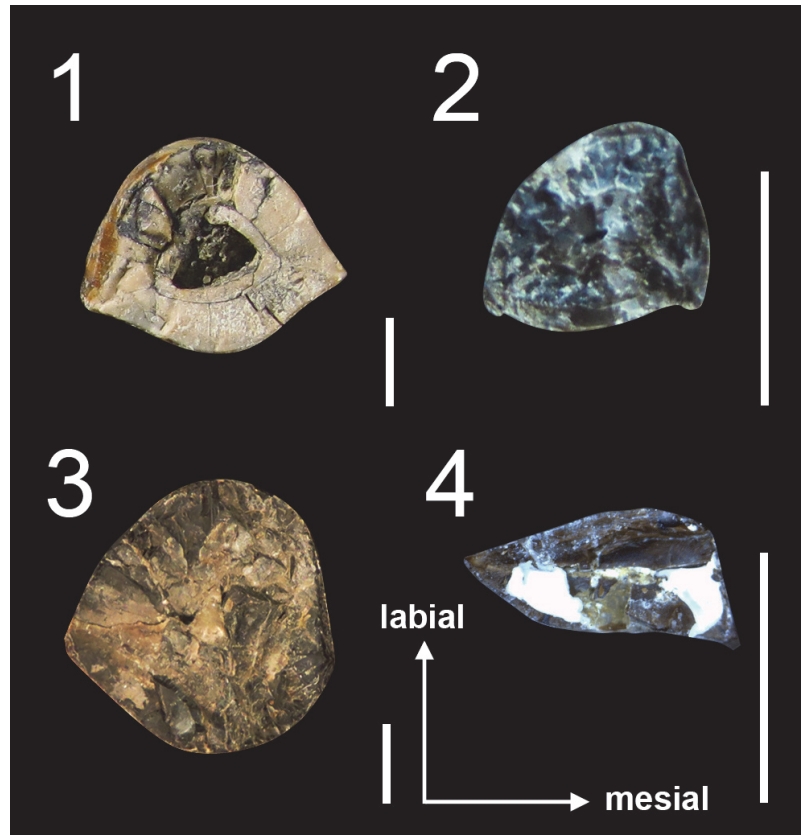

FIGURE 13. Cross-section of mesial teeth in non-avian Theropoda. 1, Salinon-shaped cross-section in the first right premaxillary tooth of the abelisaurid Majungasaurus crenatissimus (FMNH PR.2008) in apical view; 2, Ushaped cross-section in an isolated premaxillary tooth of the basal pantyrannosaurian Eotyrannus lengi (MIWG 1997.550; reversed) in apical view; 3, D-shaped crosssection in the first left premaxillary tooth of the allosaurid Allosaurus fragilis (UMNH VP 9258; reversed) in apical view; 4, J-shaped cross-section in the second right premaxillary tooth of the dromaeosaurid Atrociraptor marshalli (TMP 1995.166.01) in apical view. Scale bars equal $5 \mathrm{~mm}$.

ence of a concave surface adjacent to mesial carina on the lingual surface and, in some cases, to the distal carina on the labial surface of the crown. Such morphology of the crown is present in mesial teeth of: the noasaurid Masiakasaurus; the non-carcharodontosaurian allosauroids Allosaurus (e.g., AMNH 851, CMNH 21703) and Sinraptor (IVPP 10600); the megaraptorans Australovenator (White et al., 2015, figure 7A4, B3), Fukuiraptor (Currie and Azuma, 2006, figure 1D), Megaraptor (MUCPv 595), and Murusraptor (Coria and Currie, 2016); the tyrannosauroids Aviatyrannis (MG 27801 D90), Guanlong (IVPP V14531) and Proceratosaurus (NHMUK PV R.4860); and some paravians such as Atrociraptor (Currie and Varricchio, 2004; Figure 13.4), Deinonychus (Ostrom, 1969, figure 24D2), Dromaeosaurus (AMNH 5356; Currie et al., 1990), Linhevenator (Xu et al., 2011a), Saurornitholestes (Currie et al., 1990, figure 8.6i) and Sinornithosaurus (Xu and $\mathrm{Wu}, 2001)$. A concavity adjacent to the mesial carina on the lingual surface of the crown is also present in the lateral dentition of Masiakasaurus (FMNH PR $2471 \mathrm{dt} 4$, UA 8680 $\mathrm{dt6}$ ), some troodontids such as Xixiasaurus (Lü et al., 2010, figure 3A1), Zanabazar (Norell et al., 2009 , figure 30 , which illustrates the right mid-dentary teeth in lingual view) and an indeterminate troodontid from Uzbekistan (Averianov and Sues, 2007, figure 7L).

Remarks and synapomorphy. One or two concave surfaces marginal to the carinae appeared convergently in the mesial teeth of Abelisauroidea, non-carcharodontosaurid allosauroids, basal coelurosaurs (e.g., Ornitholestes, Guanlong, Proceratosaurus), Dromaeosauridae and Troodontinae. A salinon-shaped outline in the mesial teeth is a possible synapomorphy of Abelisauroidea and Allosauroidea (Appendix 6.9 for mesial teeth).

\section{D-shaped cross-section}

A symmetrically to asymmetrically D-shaped cross-section (Figure 1.10) can be observed in mesial teeth of some allosauroids such as Sinraptor (IVPP V10600) and Allosaurus (CMNH 1254, 21703; SMA 005/02; Figure 13.3). This morphology seems to have been lost in mesial teeth of Carcharodontosauridae, as the mesial crowns of Acrocanthosaurus (NCSM 14345), Giganotosaurus (MUCPv-CH-1, dt1), and Mapusaurus (MCFPVPH-108.166) display a mesial carina facing mesiolabially (C.H. personal obs.), forming a wide lenticular/lanceolate outline of the crown in crosssection. Although deformed, Ornitholestes mesial teeth are D-shaped in cross-section for the two distalmost premaxillary and the first two maxillary crowns (C.H. personal obs.). The neocoelurosaur Fukuivenator appears to share a relatively similar dentition, with unserrated and spatulate mesial teeth. In this taxon it is the mesialmost premaxillary tooth (or teeth) which is D-shaped in cross-section, the more distal ones being sub-oval (Azuma et al., 2016). A similar morphology was observed in the mesial crown of Falcarius (UMNH 15097), which is also unserrated and D-shaped in cross-section. A D-shaped cross-section was also noted in: a mesial isolated tooth of the noasaurid Vespersaurus (Langer et al., 2019); the premaxillary teeth of the abelisaurids Rahiolisaurus (pm1 of ISIR 550; C.H. personal obs.) and possibly an indeterminate taxon (pm1 of MPCN-PV 69; Gianechini et al., 2015, figure 2G); the megaraptoran Megaraptor (Porfiri et al., 2014); the basal ornithomimosaur Pelecanimimus (Pérez-Moreno et al., 1994); and the troodontids Byronosaurus (Averianov and 
Sues, 2016; the cross-section is, however, subcircular according to Makovicky et al., 2003), Daliansaurus (Shen et al., 2017a), Sinusonasus (IVPP V11527), Urbacodon, Xixiasaurus (Averianov and Sues, 2016), and Zanabazar (Norell et al., 2009). The cross-section at the crown-base in Megaraptor is more $\mathrm{J}$-shaped due to the presence of the spiraling mesial carina and a concave surface adjacent to it on the lingual surface of the crown (C.H. personal obs.). It is possible that unserrated and somewhat spatulate/incisiform mesial crowns with a D-shaped cross-section outline is synapomorphic to a clade gathering Fukuivenator, Ornitholestes, and maniraptoriforms, such mesial crown morphology being plesiomorphic to the basalmost members of the Therizinosauria (i.e., Falcarius) and Ornithomimosauria (i.e., Pelecanimimus and possibly Nqwebasaurus). It is unknown whether the cross-sectional outline of the mesial dentition of some troodontids such as Byronosaurus, Daliansaurus, Sinusonasus, Xixiasaurus, and Zanabazar is D-, J- or salinon-shaped but that of one premaxillary tooth of Urbacodon (CCMGE 71/12455) genuinely appears D-shaped based on the lingual profile of the crown (Averianov and Sues, 2016, figure 4B). The cross-section of the incisiform (sensu Cau et al., 2017) premaxillary teeth of the basal dromaeosaurid Halszkaraptor also appear to be D-shaped at the crown-base.

Remarks and synapomorphy. As seen with the mesial dentition of Abelisauridae and Allosauridae there is a continuum of morphological variation from a D-shaped/salinon-shaped cross-sectional outline of the first mesial crowns to a more Jshaped outline of the more distal crowns (Fanti and Therrien, 2007). The differences in cross-section morphologies are then positional and can, therefore, be subtle in theropods, so that D-shaped, salinon-shaped and J-shaped cross-sections are not discrete conditions. A J-shaped/D-shaped crosssection outline is synapomorphic to Dromaeosauridae (Appendix 6.9 for mesial teeth).

\section{U-shaped cross-section}

A U-shaped cross-section of the crown at the cervix (Figure 1.8-9) is typically referred to as Dshaped outline by several authors (e.g., Hutt et al., 2001; Sereno et al., 2009; Choiniere et al., 2010a). However, D-shaped and U-shaped cross-sections cannot be confused because the carinae of the latter are positioned on the same side of the tooth and typically facing lingually, which is not the case in a crown with a D-shaped cross-section, in which the distal carina does not face lingually $(\mathrm{C} . \mathrm{H}$. per- sonal obs.). A U-shaped cross-section of the crown base is visible in the mesial teeth of the pantyrannosaurians: Albertosaurus (Currie, 2003; Buckley et al., 2010), Appalachiosaurus (RMM 6670), Daspletosaurus (TMP 1994.153.01; Lehman and Carpenter, 1990), Dilong (IVPP V14243; Xu et al., 2004), Eotyrannus (Hutt et al., 2001; Figure 13.2), Gorgosaurus (1991.36.500), Nanotyrannus (Molnar, 1978; Carr and Williamson, 2004), Raptorex (LH PV18; Sereno et al., 2009), Tarbosaurus (Hurum and Sabath, 2003), Timurlengia (Averianov and Sues, 2012), Tyrannosaurus (Smith, 2005), Xiongguanlong (Li et al., 2010) and an indeterminate tyrannosauroid from the Cenomanian of Utah (if the mid-crown cross-section is indeed salinonshaped, the base crown is here considered $U$ shaped; Zanno et al., 2019). A similar morphology is seen in the premaxillary teeth of the non-tyrannosauroid coelurosaur Zuolong (Choiniere et al., 2010a) and in the first two premaxillary teeth of Ornitholestes (AMNH 619).

Remarks and synapomorphy. In the basal tyrannosauroids Aviatyrannis, Guanlong, and Proceratosaurus, the mesial carina of mesial teeth twists lingually but does not face entirely lingually so that the cross-section of mesial teeth is not U-shaped but rather $\mathrm{J}$-shaped. In many tyrannosauroids, the lingual surface of mesial teeth is concave, biconcave or planar, but in Ornitholestes and some tyrannosaurids like Tarbosaurus and Tyrannosaurus, the mesial margin is strongly convex, giving an oval-shaped to the crown base's cross-section (Smith, 2005, figure 8A). Due to the obvious lingual position of the carinae, the cross-sectional outline of these crowns is still referred to as U-shaped. As Dilong is currently placed as the basalmost member of the Pantyrannosauria (Brusatte et al., 2010b; Brusatte and Carr, 2016), mesial teeth with an U-shaped cross-section of the crown base is a possible synapomorphy of Pantyrannosauria (Figure 4) or a broader clade encompassing Ornitholestes, Tyrannosauroidea and Zuolong (Figure 7; Appendix 6.9 for mesial teeth).

\section{Figure-of-eight-shaped cross-section}

The presence of a figure-of-eight-shaped outline of the crown-base in cross-section, due to the presence of labial and lingual depressions on the crown base, is a common feature of dromaeosaurids such as Bambiraptor (AMNH 30556), Buitreraptor (Gianechini et al., 2011b), Dromaeosaurus (AMNH 5356), Pyroraptor (Allain and Taquet, 2000; Gianechini et al., 2011b; C.H. personal obs.), Saurornitholestes (Currie et al., 1990; Sankey et al., 
2002), Tsaagan (Norell et al., 2006) and the possible dromaeosaurid Richardoestesia gilmorei (Currie et al., 1990; Hendrickx and Mateus, 2014a). This outline, inferred from the presence of a deep labial depression on the crown and a basal depression commonly present lingually, is likely present in other dromaeosaurids such as Atrociraptor (TMP 1995.166.01), Graciliraptor (IVPP V13474), Microraptor (IVPP V16903; QM V1002; Xing et al., 2013b, figure S1) and Sinornithosaurus (IVPP V12811; Gianechini et al., 2011a). An eight-shaped cross-sectional outline is also likely present or was also observed in: the basal ceratosaur Berberosaurus (MNHN To 369); the allosaurid Allosaurus (Ldt13 of YPM-PU 14554 VII 3), the megaraptorans Megaraptor (Porfiri et al., 2014) and Orkoraptor (Novas et al., 2008); the troodontids Byronosaurus (Lmx14; Figure 6.3) and Xixiasaurus (Lü et al., 2010, figure 3A2); the tyrannosauroids Dilong (IVPP V14243), Proceratosaurus (Rauhut et al., 2010), and the two species of Alioramus, $A$. remotus (PIN 3141/1) and $A$. altai (Brusatte et al., 2012; Figure 6.2). Although large and fully mature tyrannosauroids do not appear to share this crosssectional outline (C.H. personal obs.), this dental feature does not seem to be due to immaturity as Alioramus altai, Proceratosaurus, and the Dilong specimen IVPP V14243 were subadult individuals (Xu et al., 2004; Brusatte et al., 2009b, 2012; Rauhut et al., 2010). Deep and well-visible labial and lingual depressions are also present at the base of the crown in several mid-maxillary teeth of Sinraptor (IVPP 10600), giving a figure-8-shaped outline of the crown base in cross-section.

Remarks and synapomorphy. Based on our observations, the crown base of coelophysoids, abelisauroids, megalosauroids, allosaurids, carcharodontosaurids, compsognathids, alvarezsaurids, therizinosaurs and oviraptorosaurs does not have a figure-8-shaped cross-section (C.H. personal obs.). Such an outline was also reported in the non-averostran neotheropod Liliensternus (Gianechini et al., 2011b, figure 2c), yet it is likely that the figure-8-shaped outline corresponds to a cross-section in the root base and not at the base of the crown. A figure-8-shaped cross-section of the lateral crown base is considered to be a synapomorphy of Dromaeosauridae more derived than Halszkaraptorinae (Figure 8; Appendix 6.9 for lateral teeth).

Functional morphology. The presence of a deep labial depression on the crown of the microraptorine Sinornithosaurus was interpreted as a venom delivery duct of a venomous animal by Gong et al.
(2010, 2011). This hypothesis was, however, rejected by Gianechini et al. (2011b), who did not give an alternative morphological or functional interpretation, and Turner et al. (2012). The latter consider that the labial depressions are restricted to the root, which is exposed beneath the maxilla, as the teeth are partially released from their respective alveoli as a preservational artifact. Yet, labial and lingual depressions extending on most of the crown is a common feature in dromaeosaurids (C.H. personal obs.). Both lingual and labial depressions, in fact, result from the track of the erupting replacement tooth that grows lingually from the tooth root (e.g., Acrocanthosaurus, Erlikosaurus, Falcarius, Genyodectes, Megalosaurus, Richardoestesia, Torvosaurus), linguobasally within the root, beneath the crown of the erupted tooth (e.g., Albertosaurus, Alioramus, Allosaurus, Sinraptor), or labially from the tooth root (e.g., Halszkaraptor, Megaraptor, Sinornithosaurus). The labial and lingual depressions on the root and extending apically on the crown are then the tracks of the preceding tooth abutting against the labial/ lingual surface, and the succeeding tooth in contact on the lingual/labial surface of the root. Similar to the concave surface adjacent to carinae, the presence of a lingual and/or labial depression may also have some functional implications such as strengthening the crown or allowing rapid penetration and withdrawal of the tooth from the prey.

\section{Mesial carina reaching the cervix}

The mesial carina extends to the cervix or below in many theropods. This includes: the basal saurischians Eodromaeus (PVSJ 560, 561) and Tawa (GR 241); the non-averostran theropods Coelophysis (Buckley and Currie, 2014), Dilophosaurus (UCMP 37303), Dracovenator (the mesial denticles almost extend to the cervix in the single preserved maxillary tooth; C.H. personal obs.), and Liliensternus (Cillari, 2010), and almost all abelisaurid and spinosaurid teeth. The mesial carina of a single lateral tooth of the abelisaurid Arcovenator (MHNA.PV.2011.12.187) does not seem to reach the cervix, and a few isolated baryonychine crowns bear a mesial carina extending only on half or on two-thirds of the crown height (Canudo et al., 2008). In non-abelisaurid ceratosaurs, the mesial carina reaches the root in the lateral dentition of Ceratosaurus, Masiakasaurus, and Noasaurus but the mesial teeth of Ceratosaurus and Masiakasaurus bear a mesial carina terminating above the cervix. A mesial carina reaching the root can also be seen in some lateral teeth of the piatnitzkysaurid 
Piatnitzkysaurus (PVL 4073). The mesial carina of the megalosauroid Monolophosaurus and the piatnitzkysaurid Marshosaurus extends above the cervix in the whole dentition.

The mesial carina extends on the entire crown height in allosauroids such as Allosaurus (e.g., USNM 8335; SMA 0005/02), Erectopus (MNHN 2001-4), Fukuiraptor (Currie and Azuma, 2006), and Sinraptor (IVPP 10600; ZDM T0024). This dental feature is also present in the large majority of carcharodontosaurine teeth, although the mesial carina of some Giganotosaurus lateral teeth extends slightly above the cervix (C.H. personal obs.). Only a few maxillary teeth show a mesial carina reaching the cervix in the basal carcharodontosaurid Acrocanthosaurus. A mesial carina reaching the cervix is present in some mesial and lateral teeth of: the tyrannosauroids Alioramus (MPC-D 100-1844), Daspletosaurus (Carr and Williamson, 2004), Gorgosaurus (USNM 12814), Raptorex (LH PV18), Shanshanosaurus (Currie and Dong, 2001a) and Tyrannosaurus, in which some mesial and lateral teeth have a mesial carina reaching the crown base (contra Smith, 2005; Rdt1 of CMNH 9380; Ldt7 and Rdt12 of FMNH PR.2081); the dromaeosaurids Atrociraptor (Currie and Varricchio, 2004), Dromaeosaurus (AMNH 5356) and Saurornitholestes (Currie et al., 1990; Baszio, 1997); and in the mesial and some lateral teeth of Troodon (Currie, 1987; Currie et al., 1990).

Remarks and synapomorphy. A mesial carina reaching the cervix is widespread among non-maniraptoriform theropods and is probably the ancestral condition in theropods. We only consider this dental feature to be synapomorphic for the clade Spinosauridae (Appendix 6.10 for lateral teeth). The denticulated mesial carina does not reach the cervix in: Eoraptor (PVSJ 512); the basal megalosauroids Marshosaurus and Monolophosaurus; the neovenatorids Australovenator (Hocknull et al., 2009), Murusraptor (Coria and Currie, 2016), and Neovenator (MIWG 6348); basal pantyrannosaurians (e.g., Dilong, Eotyrannus); Haplocheirus; Therizinosauria; Microraptorinae and the eudromaeosaurians Bambiraptor, Graciliraptor, and Velociraptor. The two preserved lateral teeth of the basal coelurosaur Zuolong also have mesial denticles restricted to the apical half of the crown (IVPP V15912; Choiniere et al., 2010a). Hendrickx et al. (2015c) noted that the mesial carina of all megalosaurids terminates significantly above the cervix, yet two isolated teeth referred to Torvosaurus tanneri (BYU-VP 725 12817) and T. gurneyi (ML 857) bear a mesial carina reaching the root or extending close to it. A mesial carina terminating above the root in the lateral dentition is here considered a synapomorphy of Megalosauroidea and Maniraptora (Appendix 6.10 for lateral teeth).

Functional morphology. It has been demonstrated that the extension of the denticulate mesial carina correlates with the distal curving of the crown in ziphodont theropods, the tooth curvature decreasing mesially (D'Amore, 2009). The crown area that may not contact the flesh, called the 'dead-space', tends to be unserrated, and the dead-space that is produced during the puncturing by the denticulate margins of the crown is what allows for the removal for flesh (D'Amore, 2009). The extension of the denticulated mesial carina in different clades of ziphodont and pachydont theropods conforms to this model. For instance, the mesial carina of the poorly curved lateral crowns of Abelisauridae, Baryonychinae, Carcharodontosaurinae, and Ceratosauridae almost always reaches the cervix. However, this model only applies to meat-eating dinosaurs with ziphodont, pachydont and conidont teeth. Indeed, the denticles of herbivorous theropods with folidont dentition such as therizinosaurs were probably adapted to cut through hard fibrous material, and were consequently subject to different selection pressures (D'Amore, 2009).

\section{Twisted mesial carina}

Mesial dentition. A mesial carina spiraling on the crown from the mesial side apically to the mesiolingual or lingual side basally occurs in the mesial teeth of various theropods: the non-averostran neotheropod Dilophosaurus (UCMP 37302); the noasaurid Masiakasaurus (e.g., UA 8680; FMNH PR.2182, 2471); the large majority of allosauroids such as Allosaurus (e.g., AMNH 851; NHFO 455), Australovenator (Hocknull et al., 2009, figure 20B), Fukuiraptor (Currie and Azuma, 2006, figure 1D), Mapusaurus (MCF-PVPH-108.166), Sinraptor (Currie and Zhao, 1993) and Tyrannotitan (MPEFPV 1156). A twisted carina is also present in the basal tyrannosauroids Aviatyrannis (MG 27801 D90, D172) and Proceratosaurus (NHMUK R.4860), with more derived tyrannosauroids having a straight mesial carina facing lingually. In the abelisaurids Indosuchus (AMNH 1753) and Majungasaurus (FMNH PR 2100) the mesial carina of the fourth premaxillary crown also weakly curves mesiolingually. The dromaeosaurids Deinonychus (Ostrom, 1969), Dromaeosaurus (Currie et al., 1990), Sinornithosaurus (Xu and Wu, 2001) and 
Saurornitholestes (Currie et al., 1990) also have a spiraling mesial carina.

Lateral dentition. This condition appears in the mesialmost teeth of the lateral dentition in: Allosaurus (USNM 8335; UMNH VP 9168); tyrannosauroids such as Albertosaurus (DMNH 22019), Alioramus (MPC-D 100-1844; Brusatte et al., 2012), Appalachiosaurus (Carr et al., 2005), Daspletosaurus (TMP 1994.143.01), Proceratosaurus (NHMUK PV R.4860), Raptorex (LH PV18), Tyrannosaurus (FMNH PR.2081; Smith, 2005) and Zhuchengtyrannus (Hone et al., 2011); and the dromaeosaurids Dromaeosaurus (AMNH 5356; Currie et al., 1990) and Saurornitholestes (TMP 94.12.844, TMP 88.121.39; Currie et al., 1990, figure $8.2 \mathrm{~V}$ ). In the holotype specimens of the piatnitzkysaurid Piatnitzkysaurus (PVL 4073) and the dromaeosaurine Dromaeosaurus, the mesial carina spirals onto the mesiolingual side of the crown even in more distal teeth of the lateral dentition (Currie et al., 1990; C.H. personal obs.), a dental autapomorphy in these two taxa. In Dromaeosaurus, a twisted mesial carina is, in fact, present in all teeth (Currie et al., 1990). A twisted mesial carina does not occur in the lateral dentition of Carcharodontosauria, non-avetheropod theropods and Metriacanthosauridae. In non-eudromaeosaurian neocoelurosaurs, this condition has only been observed in a few lateral teeth of the basal and derived therizinosaurs Falcarius (UMNH VP 14528; Button et al., 2017, supplemental figure S2) and Segnosaurus (the 'folded carina' sensu Zanno et al., 2016), respectively.

Remarks and synapomorphy. In Falcarius, the spiraling mesial carina, present in mesial maxillary and several dentary teeth (UMNH VP 14545; Button et al. (2017), supplemental figure S2), is unserrated in the basal half portion of the crown (n.b., the single fully erupted dentary tooth in specimen UMNH VP 14528 has most likely rotated in its alveoli so that the spiraling carina is the mesial carina; C.H. personal obs.). Unlike Falcarius, the spiraling mesial carina of Segnosaurus is denticulated along most of the carina and, in some cases, bears apicobasally tall and vertically oriented denticles creating the 'lingual folding of the mesial carina' noted by Zanno et al. (2016). Some dentary teeth of Segnosaurus also show the peculiarity of having a sporadic variation of denticle size along the mesial carina, a split distal carina, extracarinal mesial denticles, and a third denticulated carina centrally positioned on the lingual surface of the crown (Zanno et al., 2016). The combination of these autapomorphic dental features in Segnosaurus was inter- preted by Zanno et al. (2016) as indicative of increased shredding capabilities and a higher degree of oral processing than in other therizinosaurs. A bifurcated distal carina, triple carinae, and extracarinal denticles have not been observed in the dentition of other therizinosaurs or theropods, and a sporadic variation of denticle size only occurs in the conidont teeth with minute denticles of baryonychine theropods. We conclude that these dental features, with the exclusion of the spiraling mesial carina, which is also present in Falcarius, were likely caused by trauma and do not result from a highly specialized feeding strategy. A mesial carina twisting onto the crown in the lateral dentition is here considered to be synapomorphic for Tyrannosauroidea (Appendix 6.11 for lateral teeth).

Functional morphology. According to Bakker (1998), a mesial carina passing inward from the crown tip, associated with a distal carina passing outward, would keep shallow wounds open during an attack. The combination of a lingually twisted mesial carina and a strongly labially displaced distal carina would indeed enable to slice the flesh on a crown width when penetrating the prey item. This would ultimately result in wider wounds compared to bites made by labiolingually narrowed teeth with carinae positioned on the same sagittal plane. The presence of many crowns with twisted mesial carina in the lateral dentition of Allosaurus, Dromaeosaurus and tyrannosauroids seems to support the hypothesis of a predatory lifestyle rather than obligate scavenging in these theropods although further work is required to test this hypothesis.

\section{Split mesial carina}

Split mesial carinae have been reported in several Tyrannosauridae including Albertosaurus, Alectrosaurus, Daspletosaurus and Tyrannosaurus (TMP 1994.143.1; Currie et al., 1990; Erickson, 1995; Abler, 1997; Tanke and Currie, 1998; Smith, 2005; Cillari, 2010). This crown abnormality is not rare in the dentition of Tyrannosauridae, and among 993 tyrannosaurid teeth examined by Erickson (1995), $11 \%$ displayed such a feature. Outside the clade of Tyrannosauridae, a split carina has been reported from isolated theropod teeth with uncertain or broad affinities: a possible coelophysoid from the Middle Jurassic Toutunhe Formation of Liuhonggou, China (Maisch and Matzke, 2003); a possible dromaeosaurid from the MiddleUpper Jurassic Shishugou Formation of Wucaiwan, China (Morphotype 5; Han et al., 2011); a 
tyrannosauroid from the Lower Cretaceous of the Rhenish Massif of Germany (Lanser and Heimhofer, 2015); and indeterminate theropods from the Upper Cretaceous Minhe Formation of China (Bohlin, 1953), Fruitland Formation of the San Juan Basin in New Mexico, USA, and the Bauru group of Brazil (Kellner, 1996). The theropod tooth from the upper portion of the Bauru group shows a morphology reminiscent to that of abelisaurid teeth (C.H. personal obs.), and a split carina is likely to be present in this clade. A split carina has also been identified in three allosauroid theropods, Allosaurus (Erickson, 1995) and two indeterminate carcharodontosaurids from Niger (Brusatte and Sereno, 2008) and Brazil (Candeiro and Tanke, 2008). In maniraptoriforms, the dromaeosaurid Dromaeosaurus albertensis from the Upper Cretaceous Prince Creek Formation of Alaska (Fiorillo and Gangloff, 2001) and an indeterminate dromaeosaurid from the Barremian of Uña in Spain (Rauhut, 2002) also show a bifurcated mesial carina. The therizinosaur Segnosaurus galbinensis from the Upper Cretaceous Bayanshiree Formation of Mongolia (Zanno et al., 2016) has a split distal carina occurring at the crown base delimiting a flattened triangular facet. This is present from the second to the twelfth dentary tooth on both left and right dentaries (Zanno et al., 2016) and appears to be unique to this taxon. Although uniformly expressed across the dentary tooth row on both sides of the mandible, this condition is here interpreted as an abnormality possibly due to trauma.

Remarks and functional morphology. Besides the isolated tooth MNN GAD15 illustrated and ascribed to a carcharodontosaurid by Brusatte and Sereno (2008), we did not observe split carina in the dentition of any non-tyrannosaurid taxa examined first hand, suggesting that this condition is not common outside Tyrannosauridae. Erickson (1995) equates the presence of split carinae as caused by trauma, aberrant tooth replacement, or genetic factors. The presence of this abnormality in many specimens of tyrannosaurids and at least three allosauroid taxa indicates that this feature may have some taxonomic potential and should be considered in phylogenetic analyses.

\section{Distal carina strongly deflected labially.}

Whole dentition. The distal carina of mesial and some lateral teeth is strongly displaced labially (i.e., the distal carina is at the level of the labial margin of the crown in distal view) in the crowns of: the ceratosaurids Ceratosaurus (USNM 4735) and Genyodectes (MLP 26-39); the noasaurid
Masiakasaurus (UA 8680; FMNH PR.2201, 2221, 2476), and the dromaeosaurid Dromaeosaurus (AMNH 5356). In the allosauroids Allosaurus (USNM 8335; SMA 005/02) and Sinraptor (IVPP V10600), a strongly labially displaced distal carina occurs in the crowns from the distal portion of the mesial dentition and the mesial portion of the lateral dentition. In the tyrannosauroids Alioramus (MPC-D 100-1844), Gorgosaurus (USNM 12814; AMNH 5458), Proceratosaurus (NHMUK PV R.4860), Raptorex (LH PV18) and Tyrannosaurus (CMNH 9380; NHMUK PV R.7994; FMNH PR.2081), the distal carina is strongly deflected labially in mesial teeth as well as in the mesial half of the lateral (i.e., 'transitional') dentition. In tyrannosaurids, the carina is significantly labially displaced in most dentary teeth (C.H. personal obs.). Mesial and lateral dentitions. A strongly deflected distal carina towards the labial side of the crown also occurs in the mesial teeth in: the nonaverostran saurischians Dilophosaurus (UCMP 37302), Eoraptor (PVSJ 512) and Ischisaurus (MACN 18.060); the megalosauroid Monolophosaurus (IVPP 84019); the neovenatorid Fukuiraptor (Currie and Azuma, 2006, figure 1SA-B); the carcharodontosaurids Acrocanthosaurus (NCSM 14345), Giganotosaurus (MUCPv-CH-1), Mapusaurus (MCF-PVPH 108), and Tyrannotitan (MPEF-PV 1156); and the dromaeosaurids Linheraptor (IVPP V16923), Richardoestesia (Currie et al., 1990), Saurornitholestes (TMP 88.121.39) and Sinornithosaurus (IVPP V12811). A strong labial displacement of the distal carina was also noticed in some lateral teeth of: the basal ceratosaurs Saltriovenator (Dal Sasso et al., 2018, figure 5L) and Berberosaurus (MNHN To 369); the abelisaurid Arcovenator (MHNA.PV.2011.12.187); the piatnitzkysaurids Marshosaurus (UMNH VP 7824) and Piatnitzkysaurus (MACN 895); the neovenatorids Megaraptor (MUCPv 595), Neovenator (MIVG 6348) and Orkoraptor (MPM-Pv 3457); as well as Ornitholestes (AMNH 619).

Remarks and synapomorphy. The distal carina of mesial and lateral teeth is displaced lingually and not labially in the basal sauropodomorph Eoraptor (PVSJ 512). The distal carina is centrally-positioned on the crown or only weakly displaced labially in the whole dentition of Abelisauridae, Eodromaeus (PVSJ 561), Liliensternus (MB R.2175), Megalosauridae, Noasaurus (PVL 4061), Sanjuansaurus (PVSJ 605) and Spinosauridae, in the lateral dentition of Carcharodontosauridae, and possibly the whole dentition of non-dromaeosaurid neocoelurosaurs (C.H. personal obs.). The strong 
labial displacement of the distal carina is a synapomorphy of Tyrannosauroidea for both mesial and lateral teeth. Given the presence of strongly labially displaced distal carina in at least the lateral dentition of basal ceratosaurs (i.e., Saltriosaurus, Berberosaurus) and the whole dentition of ceratosaurids (n.b., the strongly displaced distal carina in both basal ceratosaurs and ceratosaurids result from the poorly convex, almost flat, labial surface of the crown, which appears to characterize all non-abelisauroid ceratosaurs; C.H. personal obs.), this dental feature is also a possible synapomorphy of the clade Ceratosauria. A centrally positioned or weakly displaced distal carina on the crown is a here considered a synapomorphy of Abelisauridae and Megalosauroidea for the whole dentition, and Carcharodontosauria for lateral teeth.

Functional morphology. A gripping function was suggested by Reichel (2012) for the mesial maxillary and dentary dentitions of Tyrannosaurus, which have crowns with a strongly labially deflected distal carina. Such placement of the distal carina, combined with the centrally positioned or lingually twisted mesial carina, causes the anterior area of the jaw to make wide cuts on the prey and consequently wide-open wounds (Reichel, 2012). Crowns with labially deflected distal carinae were accompanied by the en echelon arrangement of the mesial maxillary teeth in Tyrannosaurus, which prevented the meat from slicing forward (Reichel, 2012). Conversely, the mesial and distal carinae are centrally positioned and on the same sagittal plane in more distal crowns, providing a slicing function to these teeth (Reichel, 2012).

\section{Hooked denticles}

The presence of distal denticles with an apex pointing towards the tip (Figure 14) is a feature present in the teeth of the basal sauropodomorph Eoraptor (e.g., third right premaxillary tooth; PVSJ 512; Figure 14.1), an isolated tooth possibly belonging to the neotheropod Gojirasaurus (Carpenter, 1997; Griffin, 2019) and many abelisauroids such as Kryptops (MNN GAD1-1; Sereno et al., 2004), Majungasaurus (FMNH PR.2008, 2100, 2278; Fanti and Therrien, 2007; Figure 14.3), Masiakasaurus (FMNH PR.2221, 2296; Figure 14.2) and Rugops (MNN IGU1). Mesial and distal hooked denticles can also be observed in some therizinosaurids such as Alxasaurus (Russell and Dong, 1993; IVPP V88402; Figure 14.6), Beipiaosaurus (Liao and $\mathrm{Xu}, 2019$ ), Eshanosaurus (IVPP V11579; Figure 14.5), Jianchangosaurus (Pu et al.,
2013, figure 4E, F) and Nothronychus (Kirkland and Wolfe, 2001). Some dromaeosaurids are well known to possess apically hooked denticles (e.g., Currie et al., 1990; Currie, 1995; Larson, 2008; Longrich, 2008), yet they are only present in the eudromaeosaurians Atrociraptor (TMP 1995.166.01; Currie and Varricchio, 2004; Figure 14.7) and Saurornitholestes (Currie et al., 1990; Sankey, 2001). Finally, some anchiornithines like Caihong (Hu et al., 2018) and several derived troodontines such as Saurornithoides (Norell et al., 2009), Sinornithoides (Currie and Dong, 2001b), Troodon (e.g., Currie, 1987; Currie et al., 1990; Holtz et al., 1998; Longrich, 2008; Figure 14.8) and Zanabazar (Norell et al., 2009) also display apically hooked denticles (Makovicky et al., 2003). Among basal tetanurans, the distal denticles of some maxillary and dentary teeth (dt7 of MACN-CH 895; $\mathrm{mx} 13$ of PVL 4073; Figure 14.4) and an isolated lateral tooth associated with the holotype of Piatnitzkysaurus (PVL 4073) are strongly asymmetrically convex to apically hooked. However, the external margin of distal denticles in other maxillary and isolated lateral teeth of the holotypic and referred material of Piatnitzkysaurus floresi (e.g., mx11 of PVL 4073 and all maxillary and isolated teeth of MACN-CH 895) is symmetrically to slightly asymmetrically convex (C.H. personal obs.), showing some variation in the curvature of the external margin in the distal denticles in this taxon.

Remarks and synapomorphy. The morphology of denticles with apically hooked external margin varies significantly among theropods. In some Masiakasaurus teeth, the hooked denticles are mesiodistally narrow and differ from the more subquadrangular denticles of abelisaurids and dromaeosaurids. Likewise, denticles with hooked external margins are usually apically inclined in therizinosaurids, sometimes almost apicobasally oriented on the carinae. Nevertheless, many therizinosaurid taxa do not bear genuinely hooked denticles but rather pointed denticles perpendicular to the crown margin, as in Eshanosaurus (IVPP V11579; Zhao and Xu, 1998), or apically inclined and/or vertically oriented denticles, as seen in Beipiaosaurus (Xu et al., 1999; Liao and Xu, 2019), Erlikosaurus (Clark et al., 1994) and Jianchangosaurus (Pu et al., 2013). This is also the case in Troodontidae, which tend to have particularly large, bulbous, and widely separated denticles. Given this morphology, troodontid denticles strongly differ from the small numerous and asymmetrically convex or parallelogram-shaped denticles (Figure 14.7) of dromaeosaurids (n.b., based on this differ- 


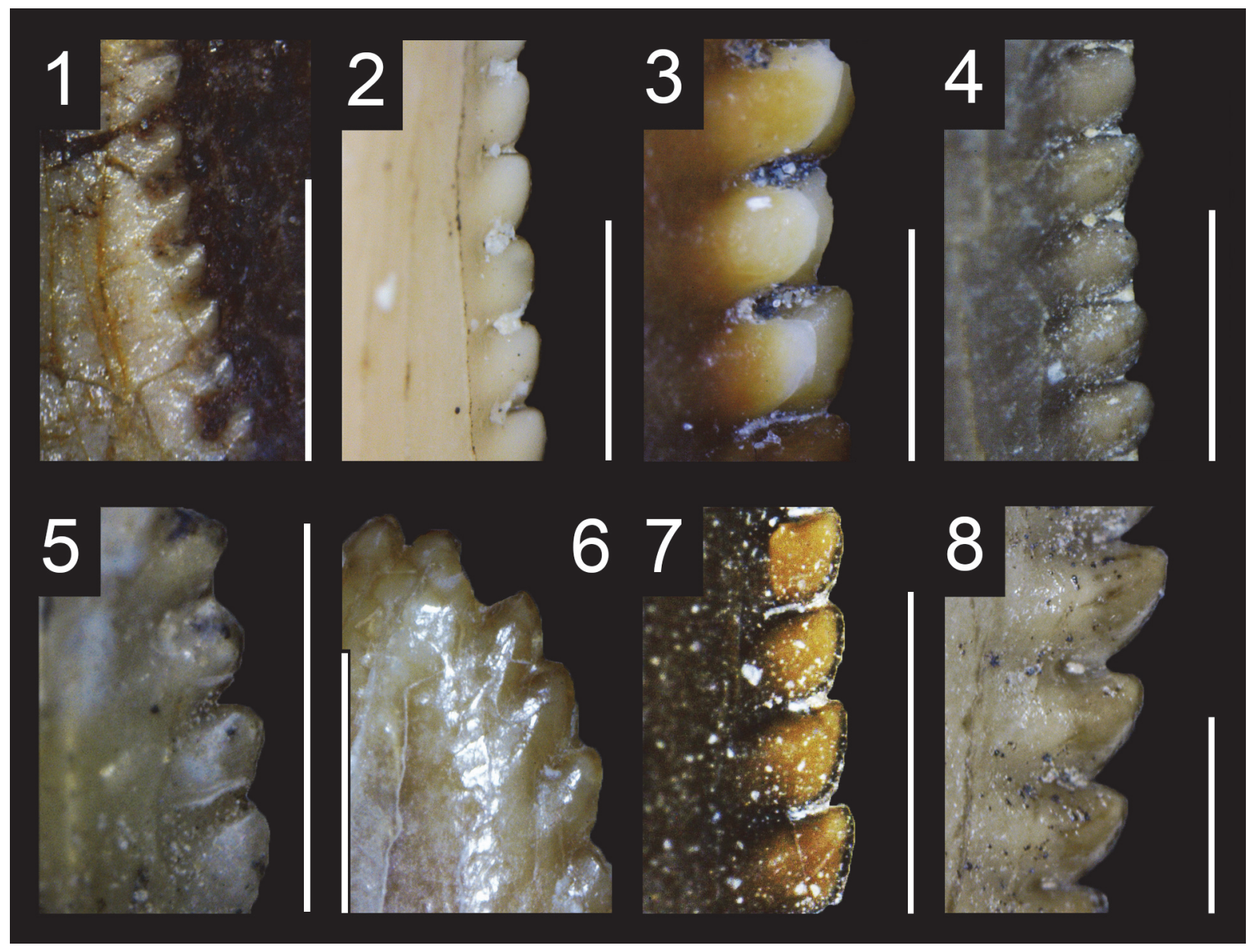

FIGURE 14. Hooked denticles in non-avian Saurischia. 1, Distal denticles of the third right premaxillary tooth of the basal sauropodomorph Eoraptor lunensis (PVSJ 512; image upside down) in labial view; 2, Distal denticles of an isolated tooth of the noasaurid Masiakasaurus knopfleri (FMNH PR.2696) in lingual view; 3, Distal carina of the second right premaxillary tooth of the abelisaurid Majungasaurus crenatissimus (FMNH PR 2008; image upside down) in lateral view; 4, Distal denticles of an isolated lateral tooth of the piatnitzkysaurid Piatnitzkysaurus floresi (PVL 4073) in lateral view; 5, Distal denticles of the sixth left dentary tooth of the therizinosaur Eshanosaurus deguchiianus (IVPP $\mathrm{V} 11579$ ) in lingual view; $\mathbf{6}$, Mesial denticles of the fifth? right dentary tooth of the therizinosaur Alxasaurus elesitaiensis (IVPP V88402; reversed) in lingual view; 7, Distal denticles of the first right maxillary tooth of the dromaeosaurid Atrociraptor marshalli (TMP 1995.166.01) in labial view; 8, Distal denticles of an isolated tooth of the troodontid Troodon formosus (DMNH 22337) in lateral view. Scale bars equal $1 \mathrm{~mm}$.

ence, the isolated teeth AMNH 8518 and $\mathrm{DMNH}$ 22870 referred to a dromaeosaurid and Saurornitholestes, respectively, are here identified as belonging to Troodon). Hooked denticles are clearly seen in Pectinodon (Longrich, 2008), Sinornithoides (Currie and Dong, 2001b), Troodon (Currie, 1987; Currie et al., 1990; Holtz et al., 1998; Ryan et al., 1998; Longrich, 2008) and some troodontid teeth from France (Vullo et al., 2007; Vullo and Néraudeau, 2010) and Central Asia (Averianov and Sues, 2007). Many troodontid taxa such as Linhevenator (Xu et al., 2011a), Pectinodon (Longrich, 2008), Saurornithoides, Zanabazar
(Norell et al., 2009), and teeth of indeterminate Troodontidae from France (Vullo and Néraudeau, 2010), Uzbekistan (Averianov and Sues, 2007) and India (Goswami et al., 2013) also show large denticles either with a pointed or a parabolic and rounded external margin. Variation in denticle sizes and morphologies may, however, be positional, as proposed by Longrich (2008) for Pectinodon bakkeri.

In many theropod clades such as ceratosaurids, megalosauroids, allosauroids and tyrannosauroids, the denticles are symmetrically rounded or asymmetrically convex in lateral view (Currie et al., 
1990; Abler, 1992; C.H. personal obs.), but never pointed or hooked apically (contra Bakker and Bir (2004) for ceratosaurids and allosaurids, and Smith (2007) for tyrannosaurids). All microraptorines such as Graciliraptor (IVPP V2339), Microraptor (CAGS-20-7-004) and Sinornithosaurus (IVPP V12811), as well as most eudromaeosaurians belonging to the clade Dromaeosaurinae + Velociraptorinae (sensu Evans et al., 2013) like Acheroraptor (Evans et al., 2013), Bambiraptor (AMNH 30556), Linheraptor (Xu et al., 2015a), Tsaagan (Norell et al., 2006), Utahraptor (Kirkland et al., 1993) and Velociraptor (AMNH 6515), do not have apically hooked denticles but instead possess symmetrically to asymmetrically convex serrations. The presence of hooked and/or pointed denticles is considered a synapomorphy of Abelisauridae, Therizinosauroidea, and Troodontinae more derived than Byronosaurus (Appendix 6.14 for lateral teeth).

Functional morphology. Hooked denticles in the dromaeosaurid Saurornitholestes were interpreted as being adapted to slicing flesh off bones by Currie et al. (1990). According to Fowler et al. (2011a), hooked denticles in dromaeosaurids are, however, not well-suited for tearing through flesh. They would instead enhance the effectiveness of the jaw's grip on the prey or helped to remove feathers and fur from prey items (Fowler et al., 2011a). Clearly, the origin of this structure is distinct from that of therizinosauroids and troodontids, being only superficially morphologically convergent. FEA on a troodontid tooth with apically hooked denticles has recently revealed that these asymmetric denticles best distribute stress when the force is directed parallel to the long axis of the denticles (Torices et al., 2018). This suggests that troodontids with coarse and apically hooked denticles were not well-adapted to acquiring struggling prey and likely favored small and softer prey with thinner bones (Torices et al., 2018). Conversely, the comparatively small and mildly apically hooked denticles of Saurornitholestes showed lower overall stress in a wide range of cutting angle than those of the troodontid crown. This indicates that, like the teeth of Dromaeosaurus with symmetrically convex denticles, the hooked denticles and overall dentition of Saurornitholestes were able to sustain powerful bite-force, so that these two dromaeosaurids were well-adapted for biting through bones and feeding on struggling preys (Torices et al., 2018). Although distantly related, abelisauroids and dromaeosaurids have similar denticle morphologies which have likely converged morphologically as a result of selection to perform a similar function. Consequently, abelisauroids were probably able to handle struggling prey and processing bones as part of their diet, a hypothesis that requires to be tested with FEA techniques in the future.

\section{Small number of denticles on the carina}

Farlow et al. (1991) demonstrated that denticle density decrease allometrically with basal crown length and height in theropods, a rule that can certainly apply to most theropods with ziphodont (D'Amore and Blumenschine, 2012) and pachydont dentition such as tyrannosaurids (Carr and Williamson, 2000), but not to folidont and conidont theropods. Indeed, some folidont theropods like therizinosaurs have small crowns with particularly coarse denticles (Clark et al., 2004) whereas others with conidont teeth like baryonychines have comparatively large crowns with minute denticles (Mateus et al., 2011; see next section; D'Amore and Blumenschine, 2012). A small number of denticles (here arbitrarily defined as less than 15 denticles on the crown) along the whole carina characterizes many troodontids such as Daliansaurus (Shen et al., 2017a), Linhevenator (Xu et al., 2011a), Pectinodon (Carpenter, 1982; Larson and Currie, 2013), Saurornithoides (Norell et al., 2009), Sinornithoides (Currie and Dong, 2001b), Sinusonasus (IVPP V11527), Troodon (Leidy, 1856; Russell, 1948; Currie, 1987) and Zanabazar (Norell et al., 2009). This feature is also present in most therizinosauroids such as Alxasaurus (Russell and Dong, 1993), Beipiaosaurus (Xu et al., 1999; Liao and Xu, 2019), Erlikosaurus (MPC-D 100-111; Clark et al., 1994), Eshanosaurus (Xu et al., 2001), Jianchangosaurus (Pu et al., 2013), Nothronychus (Kirkland and Wolfe, 2001) and Segnosaurus (MPC-D 100-80). A carina bearing few denticles is, however, not restricted to these two clades, as this dental feature can also be found in some lateral teeth of the basal oviraptorosaur Incisivosaurus (IVPP V13326), and the dromaeosaurids Microraptor (IVPP V13475; Hwang et al., 2002, figure 5) and Paronychodon (Currie et al., 1990), which has been interpreted as being a tooth morphotype of Richardoestesia by Longrich (2008) and as a pathological specimen of already known dromaeosaurid and troodontid taxa (Hwang, 2005). Some crowns of Saurornitholestes appear to have less than 15 denticles on the carina (Currie et al., 1990; Baszio, 1997; Larson and Currie, 2013) but the quantitative data gathered by Larson and Currie (2013) indicates that the large 
majority of Saurornitholestes teeth have much more than 15 denticles on the crown.

Remarks and synapomorphy. Embryonic or juvenile theropods tend to bear few coarse denticles such as the theropod embryos tentatively ascribed to Lourinhanosaurus (Araújo et al., 2013), the hatchling Scipionyx (Dal Sasso and Maganuco, 2011), and the posthatchling Sciurumimus (Rauhut et al., 2012). The presence of crowns with less than 15 denticles on the carina is a synapomorphy of Therizinosauroidea among Therizinosauria (sensu Zanno, 2010a), and Troodontidae more derived than Sinovenator (Appendix 6.16 for lateral teeth).

Functional morphology. In mature individuals, strongly constricted subsymmetrical crowns bearing relatively few large pointed denticles seems to be adapted to an omnivorous diet including plant material at least partially. Therizinosaur teeth are convergent with those of basal sauropodomorphs and iguanas (Barrett, 2000). Indeed, both possess relatively few and large pointed denticles on the carinae, mesial and distal carinae not reaching the cervix, and a convex margin of the crown, a tooth morphology that is correlated with omnivorous diets (Barrett, 2000). Troodontids with large apically hooked denticles on the distal carina have also been interpreted as omnivorous, as well as insectivorous based on the convergent dentition with iguanids and bat-eared fox, respectively (Varricchio, 1997; Holtz et al., 1998; Zanno et al., 2009). Holtz et al. (1998) suggested that the large denticles of troodontids and therizinosaurs would sever larger-sized and/or more resistant structures such as plant fibers. However, a predominantly carnivorous diet in troodontids has been inferred by other authors due to interdenticular sulci and sharp and hooked denticles (Currie and Dong, 2001b; Zanno and Makovicky, 2011; see previous sections on dentition type and hooked denticles). According to Currie et al. (1990), the large denticles of troodontids would slice through soft material and bones. Few troodontids actually possess sharply pointed and hooked serrations, and the denticles of Linhevenator (Xu et al., 2011a), Pectinodon (Carpenter, 1982; Larson and Currie, 2013), Saurornithoides and Zanabazar (Norell et al., 2009) rather display a rounded external margin. However, the presence of ziphodont lateral crowns and distally recurved pointy folidont teeth showing a weak constriction in the dentition of these troodontids support the fact that they fed predominantly on meat. Interestingly, large denticles are typically associated with a constricted crown, as illustrated with Microraptor, the only established dromaeosaurid possessing a small number of denticles on the crown and a constriction at the crown base. This suggests that Microraptor had an atypical diet among dromaeosaurids, which are usually considered as unquestioned carnivores (Norell and Makovicky, 2004; Zanno and Makovicky, 2011). The peculiar dentition of Microraptor seems to support a diverse diet, in which gut contents already revealed remains of mammals (Larsson et al., 2010), enantiornithine birds (O'Connor et al., 2011), and fish (Xing et al., 2013b). Microraptor and troodontids with denticulated teeth share the same heterodont lateral dentition made of weakly constricted teeth and a few distally denticulated crowns in the distal portion of the jaw (Hwang et al., 2002). It is, therefore, likely that derived troodontids bearing crowns with a small number of denticles were opportunistic carnivores feeding on a large variety of small prey items. As already mentioned in the previous section on hooked denticles, it was revealed that troodontids with coarse denticles likely favored small and softer prey with thinner bones (Torices et al., 2018).

\section{Large number of denticles on the carina}

Minute denticles relative to tooth size (i.e., more than 250 denticles along the distal carina) are found in the dentition of the baryonychines Baryonyx (150-300 denticles on the carinae; $~ 35$ de/ 5mm; NHMUK PV R.9951; ML 1190; Figure 15.1) and Suchomimus (100-280 denticles on the carinae; 35 de/5mm; e.g., UC G67-1, G22-7, G34-12; Figure 15.2) on both carinae and, due to their large size, some lateral teeth of the carcharodontosaurid Acrocanthosaurus (80-270 denticles on the carinae; 15 de/5mm; NCSM 14345; Smith et al., 2005).

Remarks and synapomorphy. A distal carina with a large number of denticles (i.e., more than 200 denticles) is also present in some particularly apicobasally long lateral crowns of the ceratosaurid Ceratosaurus ( 15 de/5mm; ML 865, 1151; Smith et al., 2005) and the tyrannosaurid Tyrannosaurus ( 10 de/5mm; Smith, 2005). Regardless of the tooth dimension, theropods with particularly small denticles, i.e., more than 6 denticles per millimeter on the distal carina, include non-neotheropod saurischians such as Aorun, Baryonychinae, Coelophysis, Compsognathidae, Eodromaeus, Eoraptor, Falcarius, Liliensternus, Noasauridae, Ornitholestes, Proceratosaurus, Richardoestesia, Saurornitholestinae (including Saurornitholestes), Sinovenator and Velociraptor (Smith et al., 2005; 


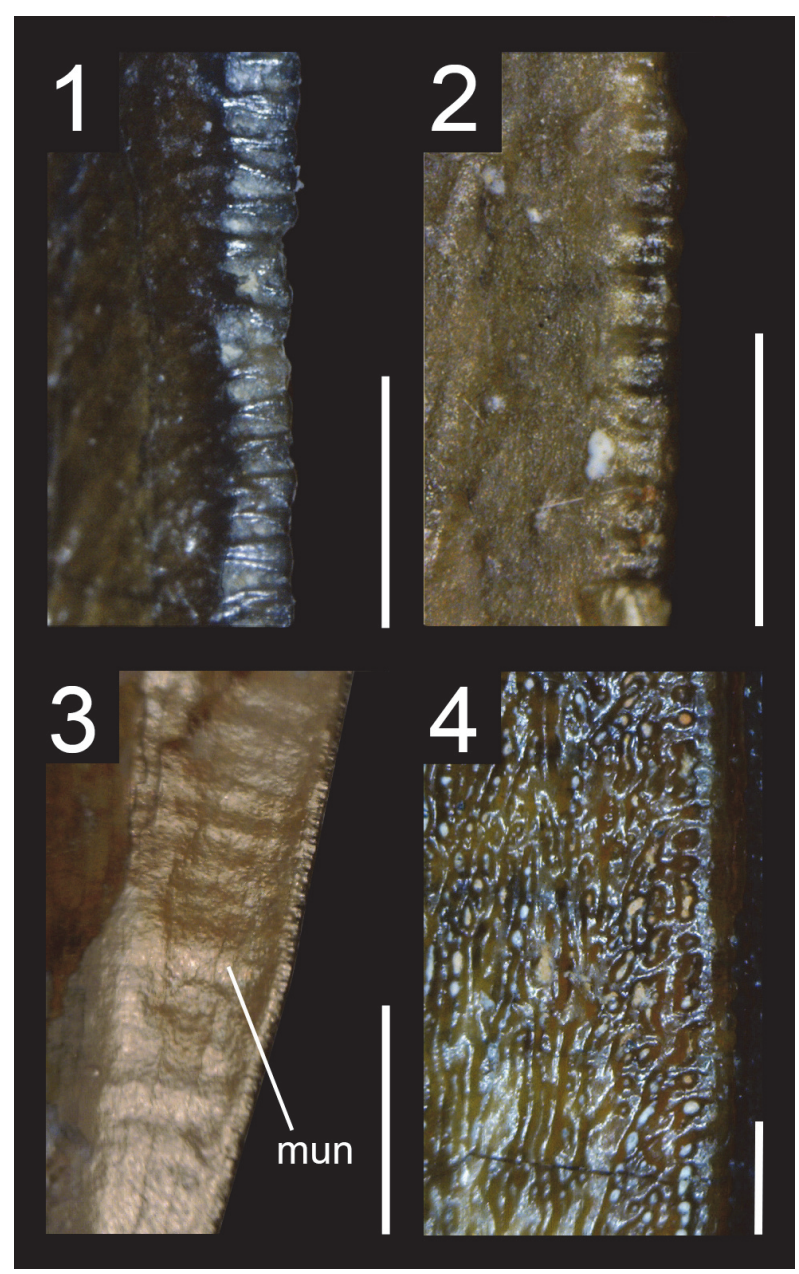

FIGURE 15. Denticles and carinae in Spinosauridae. 1, Carina and denticles of an isolated tooth of Baryonyx walkeri (NHMUK PV R.9951 R.278) in lateral view with details on the basally curving and veined enamel surface texture; 2, Carina and denticles of an isolated tooth of Suchomimus tenerensis (MNN G73-3) in lateral view; 3, Maxillary tooth of Irritator challengeri (SMNS 58022) in labial view showing the marginal undulations and the 'beaded' carina; 4, Isolated tooth of Spinosaurus cf. aegyptiacus (MSNM V6422) in lateral view, showing the 'beaded' carina and the anastomosed enamel surface texture. Abbreviations: mun, marginal undulation. Scale bars equal $5 \mathrm{~mm} \mathrm{(3),} 1 \mathrm{~mm}(1-2,4)$.

Zanno, 2010b; Rauhut et al., 2012; Larson and Currie, 2013; Choiniere et al., 2014a; C.H. personal obs.). The presence of a large number of minute denticles (more than $25 \mathrm{de} / 5 \mathrm{~mm}$ for crowns of more than $4 \mathrm{~cm}$ ) along both carinae is a synapomorphy of Baryonychinae (Appendix 6.16 for mesial and lateral teeth).

Functional morphology. The presence of minute denticles in large baryonychine teeth seems to be correlated with the increase in robustness of the crown (Charig and Milner, 1997). It may also result from the simplification of teeth that were mostly used to impale prey rather than slicing their flesh, evolving towards unserrated crowns in Spinosaurinae (Charig and Milner, 1997; Buffetaut, 2011; Gianechini et al., 2011b). This dental simplification is also present in other theropods such as Compsognathus, in which many teeth have lost serrations. The reversed condition occurred in Therizinosauria and Troodontidae, in which the basal forms bear minute denticles that increase in size in more derived taxa. Minute denticles are not suitable for the 'rip and grip' cutting action of medium-sized serrations of most non-avian theropods and might function in a similar way to unserrated teeth (Farlow et al., 1991; Charig and Milner, 1997).

\section{Sporadic variation of denticle size}

Random variation of denticle size along denticulated carina (Figure 15.1-2) has been observed in the baryonychines Baryonyx (NHMUK PV R.9951, ML 1190; Mateus et al., 2011; Figure 15.1) and Suchomimus (MNN G26-5b; Figure 15.2), and was thought to be restricted to Baryonychinae (Mateus et al., 2011). This dental feature is, however, present on the mesial carina of an isolated mesial tooth of Nuthetes (NHMUK PV R.15871 B), teeth referred to Richardoestesia isosceles (Larson, 2008) and some dentary crowns of the therizinosaurid Segnosaurus. In the latter, one small denticle is bounded by two large ones (Zanno et al., 2016, figure $3 \mathrm{~A}$ ).

Remarks and synapomorphy. In spinosaurids, a sporadic variation of denticle size is much more developed in Baryonyx and occurs along the whole carinae in this taxon. In Suchomimus, most denticles gradually change in size along the carinae and the basal part of the carina only displays this sporadic change of denticle size. Such a feature most likely results from the reduction of denticle dimension in Spinosauridae, which occurred throughout the evolution of this clade. In Spinosaurinae, the carina is unserrated, yet it does not correspond to a smooth and regularly shape ridge. In lateral view, the carinae of Irritator and Spinosaurus are 'beaded' (sensu Sues et al., 2002; Figure 15.3) and sculptured (Figure 15.4), respectively (Sues et al., 2002; Hasegawa et al., 2010), thus witnessing the vestigial presence of small denticles along the carinae. The sporadic variation of denticle size observed in Segnosaurus is, here, interpreted as an abnormality caused by trauma (see above). A sporadic variation of denticle size along the carinae 


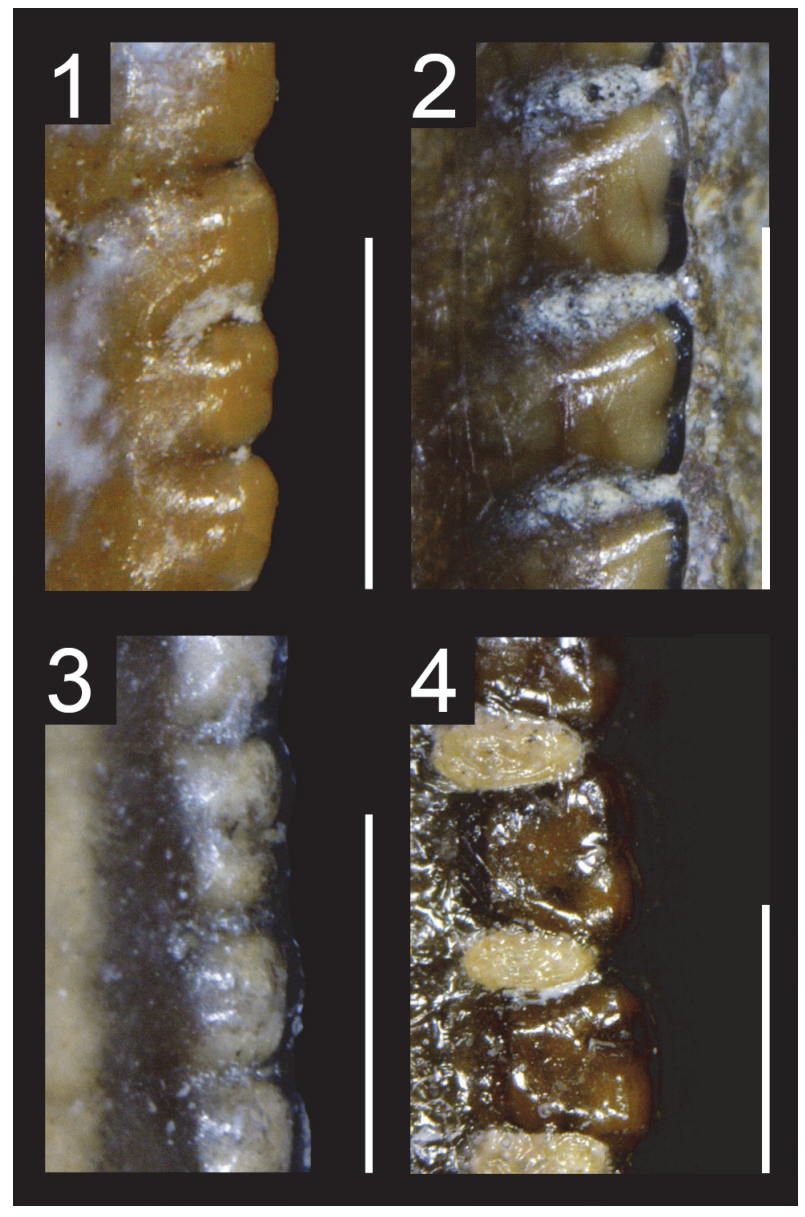

FIGURE 16. Bilobate denticles in non-avian Theropoda. 1, Mesial carina of an isolated crown of the abelisaurid Aucasaurus garridoi (MCF-PVPH 236) in lateral view; 2, Mesial carina of an isolated tooth of the megalosaurid Megalosaurus bucklandii (NHMUK PV R.234; tooth in matrix) in lateral view; 3, Mesial carina of the third left maxillary tooth of the putative metriacanthosaurid Erectopus superbus (MNHN 2001-4) in labial view; 4, Mesial carina of the tenth maxillary tooth of the tyrannosaurid Tyrannosaurus rex (FMNH PR.2081) in labial view. Scale bars equal $1 \mathrm{~mm}$.

is synapomorphic for Baryonychinae (Appendix 6.17).

\section{Bilobate denticles}

Thought to be an autapomorphy of the carcharodontosaurid Tyrannotitan chubutensis (Novas et al., 2005; Canale et al., 2015), denticles with a biconvex external margin (Figure 16) have been observed in several theropod clades, typically in the apical part of the mesial carina. Bilobate denticles have been identified in: the megalosaurids Duriavenator (NHMUK PV R.332; Figure 16.2), Megalosaurus (OUMNH J.13506; NHMUK PV
R.234) and possibly Torvosaurus (ML 1100); the carcharodontosaurids Acrocanthosaurus (SMU 74646) and Carcharodontosaurus (UCRC PV6); the possible metriacanthosaurid Erectopus (MNHN 2001-4; Figure 16.3), and the tyrannosaurid Tyrannosaurus (FMNH PR.2081, $\mathrm{mx10}$; Figure 16.4). They also appear to be present in the abelisaurids Abelisaurus (MPCA 5) and Aucasaurus (MCFPVPH 236; Figure 16.1), although the double convexity may result from the external margin being slightly worn out. Bilobate denticles have also been observed on the teeth of non-theropod dinosaurs such as the rauisuchid Postosuchus (R. Molnar, personal commun., 2017).

Remarks and synapomorphy. Bilobate denticles seem to be malformations possibly resulting from trauma. Yet, they have not been observed in any non-averostran theropods and Maniraptoriformes and may, therefore, correspond to a tooth trait change due to genetic factors influencing denticle morphology, a hypothesis that requires further testing. We, however, do not consider the presence of bilobate denticles as an apomorphic feature of a certain clade or taxon.

\section{Size difference between mesial and distal denticles}

Distal larger than mesial denticles. Long thought to characterize the dentition of Dromaeosauridae, and therefore used as a primary feature to identify dromaeosaurid teeth (e.g., Rauhut and Werner, 1995; Sweetman, 2004; Vullo et al., 2007; Lubbe et al., 2009), the difference in size between mesial and distal is widespread among other clades. Subtle differences in size may occur between mesial and distal denticles, and we consider there is a significant size variation between the mesial and distal denticulated carinae when the denticle size index (DSDI) is higher than 1.2 or lower than 0.9 . The arbitrary value of 1.2 to explain the much larger size of distal denticles compared to the mesial ones, was proposed by Rauhut et al. (2010) and corresponds to more than six mesial denticles for five distal serrations. A DSDI higher than 1.2 was measured in the lateral dentition of: the nonaverostran neotheropods Dracoraptor (NMW 2015.5G), Dracovenator (BP/1/5243; Yates, 2005), and Dilophosaurus (Welles, 1984; Smith et al., 2005; UCMP 37302), but not Sinosaurus triassicus (IVPP V34; = 'Dilophosaurus' sinensis according to Xing et al., 2013a); the noasaurids Noasaurus (PVL 4061) and Masiakasaurus (e.g., UA 9091; FMNH PR.2201, 2211, 2696; Figure 17.1); the abelisaurids Arcovenator (MHNA.PV.2011.12.15; 


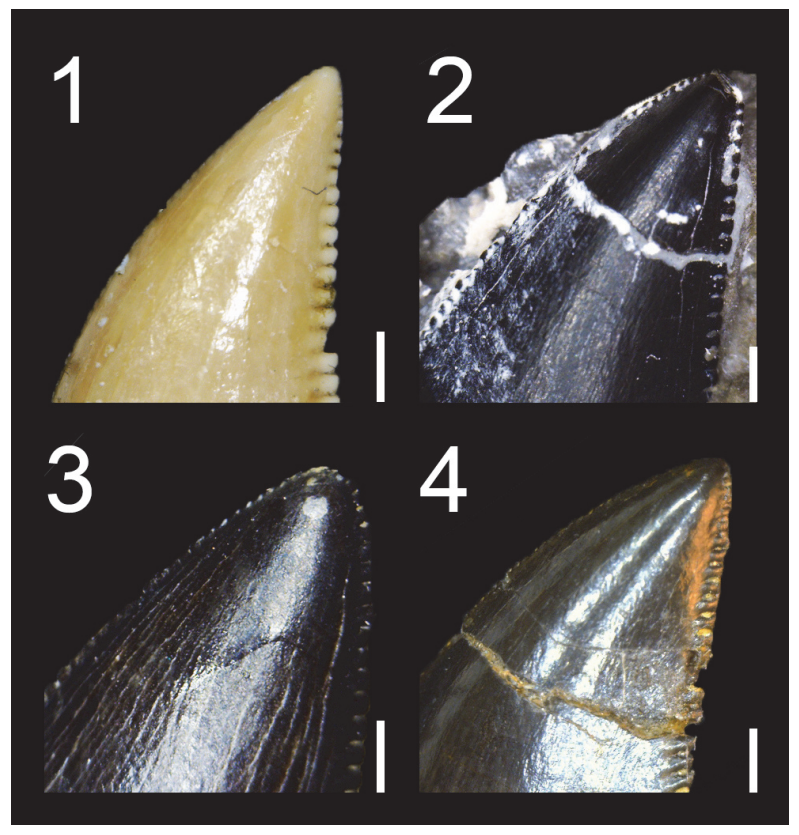

FIGURE 17. Distal larger than mesial denticles in nonavian Theropoda. 1, Isolated lateral crown of the noasaurid Masiakasaurus knopfleri (FMNH PF 2221) in labial view; 2, Fifth right dentary crown of the piatnitzkysaurid Marshosaurus bicentesimus (UMNH 6368) in lingual view; 3, First left maxillary crown of the basal pantyrannosaurian Eotyrannus lengi (MIWG 1997.550) in lingual view; 4, Distal dentary crown of the dromaeosaurid Deinonychus anthirrhopus (YPM 5232 612) in lingual view. Scale bars equal $1 \mathrm{~mm}$.

MHNA.PV.2011.12.187) and Majungasaurus (Fanti and Therrien, 2007); and the piatnitzkysaurids Marshosaurus (Madsen, 1976; UMNH 6368; Figure 17.2) and Piatnitzkysaurus (PVL 4043; MACN-CH 895), but not Condorraptor (Rauhut, 2005). A difference in size between mesial and distal denticles was also measured in a few teeth of: the baryonychines Baryonyx (NHMUK PV R.9951) and Suchomimus (UC G26-5; UC G67-1); the mesial dentition of Acrocanthosaurus (NCSM 14345) and Ceratosaurus (UMNH VP 5278), and in some lateral teeth of Allosaurus (e.g., UMNH VP 6189, UMNH VP 5841). Most crowns of these taxa appear, however, to have a DSDI close to 1 (C.H. personal obs.).

As noted by Rauhut et al. (2010), this condition is widespread among basal Tyrannosauroidea and can be observed in Dilong (Xu et al., 2004), Eotyrannus (Hutt et al., 2001; MIWG 1997.550, $\mathrm{mx} 1$ with DSDI of 1.5 , isolated lateral crown with DSDI of 1.35; contra Sweetman, 2004; Figure 17.3), Guanlong (Xu et al., 2006), Proceratosaurus (Rauhut et al., 2010), Stokesosaurus (UMNH VP
6368), Timurlengia (Averianov and Sues, 2012) and Xiongguanlong (Li et al., 2010). The proceratosaurid Yutyrannus (ELDM V1001) has mesial and distal denticles of the same size at mid-crown, whereas the tyrannosaurid Nanotyrannus, a possible juvenile of Tyrannosaurus (e.g., Carr, 1999; Carr and Williamson, 2004; see Larson, 2013 for a different opinion), shows a DSDI higher than 1.2 in most teeth (Carr and Williamson, 2004).

Among non-tyrannosauroid coelurosaurs, A DSDI higher than 1.2 was also measured in the teeth of the basal coelurosaurs Bicentenaria (MPCN-Pv 866) and Zuolong (IVPP V15912, premaxillary tooth), as well as the therizinosaurs Falcarius (UMNH VP 14528 Ldt?) and Nothronychus (Hedrick et al., 2015). Nonetheless, no size discrepancy between mesial and distal denticles was noticed in the dentition of Erlikosaurus (Zanno et al., 2016, figure 4), Eshanosaurus (IVPP V11579), Segnosaurus (Zanno et al., 2016), and the maxillary teeth of Falcarius (UMNH VP 14545). The majority of eudromaeosaurians such as the microraptorines Changyuraptor (Han et al., 2014), Graciliraptor (Xu and Wang, 2004), Sinornithosaurus (Xu and Wu, 2001) and Tianyuraptor (Zheng et al., 2010), the dromaeosaurines Achillobator (Perle et al., 1999), Atrociraptor (Currie and Varricchio, 2004), Dakotaraptor (DePalma et al., 2015) and Utahraptor (BYU-VP 18075), the velociraptorines Acheroraptor (Evans et al., 2013), Bambiraptor (Burnham, 2004), Deinonychus (Ostrom, 1969; Figure 17.4), Linheraptor (IVPP V16923), Saurornitholestes (Currie et al., 1990), and Velociraptor (Barsbold and Osmólska, 1999), and the European dromaeosaurids Nuthetes (Milner, 2002) and Pyroraptor (Allain and Taquet, 2000) also have a DSDI higher than 1.2. In Troodontidae, the lateral teeth of Zanabazar (Barsbold, 1974) and some isolated crowns assigned to Troodon also display such difference in denticle size between the mesial and distal carinae (Currie, 1987, figure 5k, m). Mesial denticles significantly smaller than distal denticles were also noted in the basal neotheropod Liliensternus (Smith et al., 2005), but both carinae bear denticles of relatively similar size in this taxon (Cillari, 2010; C.H. personal obs.).

Mesial larger than distal denticles. Mesial denticles are significantly larger than distal denticles (DSDI < 0.9) at mid-crown in some lateral teeth of: the basal sauropodomorph Eoraptor (PVSJ 512); the megalosaurids Afrovenator (UC UBA 1), Duriavenator (NHMUK PV R.332), Megalosaurus (NHMUK PV R.234; Milner, 2002), and Torvosaurus (ML 148); the carcharodontosaurids Acrocan- 
thosaurus (NCSM 14345), Carcharodontosaurus (SGM Din-1; MNN GAD12), Eocarcharia (MNN GAD13, GAD14), Giganotosaurus (MUCPV-CH-1) and Mapusaurus (MCF-PVPH-108.9); the tyrannosaurids Gorgosaurus (ROM 1247; Smith et al., 2005), Tyrannosaurus (UCMP 118742; Carr and Williamson, 2004; Smith et al., 2005) and Zhuchengtyrannus (IVPP FV 1794); as well as the dromaeosaurid Dromaeosaurus (AMNH 5356).

Remarks and synapomorphy. A significant difference in size between mesial and distal denticles, with the mesial serrations being significantly smaller than the distal ones, was acquired independently in Abelisauridae, Dilophosauridae, Dromaeosauridae, Noasauridae, Piatnitzkysauridae, and Tyrannosauroidea, and teeth bearing distal denticles significantly bigger than the mesial ones are considered as synapomorphic for the clades Coelurosauria, Noasaurinae, and Piatnitzkysauridae (Appendix 6.19 for lateral teeth). Likewise, the presence of some lateral teeth with mesial denticles significantly larger than distal ones was convergently acquired by large-sized megalosaurids, carcharodontosaurids, and tyrannosaurids, as well as the dromaeosaurid Dromaeosaurus.

Functional morphology. According to Fowler et al. (2011a), the reduction of mesial denticles would enhance the piercing function of the tooth. However, this functional hypothesis has never been tested. Distal denticles significantly larger than mesial ones are typically present in small-sized meat-eating theropods (i.e., Noasauridae, basal Tyrannosauroidea and Eudromaeosauria). The mesial and distal denticles are subequal in size in Utahraptor (Kirkland et al., 1993) and only slightly smaller on the mesial carina in Achillobator (Perle et al., 1999), the two largest dromaeosaurids. Piatnitzkysauridae and derived Abelisauridae appear to be the only medium-sized theropods showing a clear difference in size between mesial and distal serrations, with mesial denticles being significantly smaller than the distal ones. Mesial denticles larger than distal denticles tend to be present in particularly large-sized theropods such as megalosaurids, carcharodontosaurids and tyrannosaurids. Consequently, functional or developmental factors, or a combination of both, seem to come into play for the establishment of this condition in such disparate theropod clades with possibly similar feeding strategies.

Distal denticles significantly larger than mesial ones, in fact, appear to be a common feature of juvenile tyrannosaurids, with DSDIs decreasing progressively throughout ontogeny in at least
Tyrannosaurus (Carr and Williamson, 2004). A significant difference between mesial and distal denticles has for instance been observed in the transitional dentition (i.e., teeth at the transition between the mesial and lateral dentition) of an immature specimen of Daspletosaurus (TMP 1994.143.01), the premaxillary, maxillary and dentary dentitions of a juvenile Tarbosaurus (Tsuihiji et al., 2011), and the lateral dentition of the juvenile tyrannosaurine Shanshanosaurus (Currie and Dong, 2001a). In the young specimens of Daspletosaurus, the carinae of the premaxillary teeth are unserrated (TMP 1994.143.1; Currie, 2003) and show the beaded condition seen in Irritator (Figure 15.3). Transitional teeth in this taxon bear minute and, in some cases, poorly delimited mesial denticles and much larger and well-differentiated distal denticles.

\section{Straight to convex distal profile}

Although a straight or convex distal margin of the crown is the most common condition in conidont and folidont teeth, most ziphodont and pachydont teeth are usually slightly to strongly concave distally (Ezcurra, 2009; C.H. personal obs.). A straight or slightly curved distal profile was considered to be a synapomorphy for Abelisauridae by Smith (2007) as this feature is present in the crowns of Aucasaurus, Indosuchus, Majungasaurus, Rugops, Kryptops (Smith and Lamanna, 2006; Smith and Dalla Vecchia, 2006; Candeiro, 2007; Smith, 2007; C.H. personal obs.), and many indeterminate abelisaurids (e.g., UCPC 10; MNHN MRS1619, MRS1620). However, a straight or slightly concave curvature of the distal profile also occurs in the basalmost sauropodomorph Eoraptor (PVSJ 512), the ceratosaurids Ceratosaurus (USNM 4735) and Genyodectes (MLP 26-39), the noasaurids Noasaurus (PVL 4061), the allosaurid Allosaurus (NHFO 455), the metriacanthosaurids Sinraptor (IVPP 10600) and Yangchuanosaurus (CV 00215), the carcharodontosaurids Carcharodontosaurus (SGM Din1) and Mapusaurus (MCF$\mathrm{PVH}$ 108.43), the basal pantyrannosaurian Eotyrannus (MIWG 1997.550), and some coelurosaurs such as Paronychodon (Currie et al., 1990, figure 8.5A) and Zapsalis [Sankey et al., 2002, figure 4.10; n.b., Zapsalis corresponds to 'Dromaeosaurus' Morphotype A of Longrich (2008), ?Dromaeosaurus morphotype A of Sankey et al. (2002) and Sankey (2008), and Dromaeosaurinae morphotype A of Larson (2008)]. A straight distal margin of the crown is also present in pachydont teeth of some tyrannosaurids such as Gorgosaurus 
(USNM 12814) and Tyrannosaurus (FMNH PR.2081). A sigmoid outline of the distal margin of the crown, with the basal half slightly concave and the apical half weakly convex, is typical of carcharodontosaurid lateral teeth and can be observed in Carcharodontosaurus (SGM Din-1) and Giganotosaurus (MUCPv CH1). Among nonavian theropods, a convex distal profile of the crown is visible in some Abelisauridae, Alvarezsauroidea, Ceratosaurus, Carcharodontosaurus, Fukuivenator, Ornithomimosauria, Oviraptorosauria, Therizinosauria and Spinosaurinae.

Remarks and synapomorphy. Given the wide distribution of a straight or convex distal profile, this feature cannot be used solely to identify teeth and therefore the synapomorphy proposed by Smith (2007) cannot be supported. However, a convex distal profile of the crown is a rare condition in ziphodont teeth and is only present, to our knowledge, in Abelisauridae and a few lateral teeth of Carcharodontosaurus (Stromer, 1931, figure 2) and Ceratosaurus, in which the feature is considered to be apomorphic. A slightly convex and almost straight mesial profile is seen in Alvarezsauridae/ Parvicursorinae, Fukuivenator, Ornithomimosauria, Therizinosauria and some Spinosaurinae, whereas all other theropods display a strongly convex mesial profile. The presence of some lateral crowns with a straight distal profile is here considered to be synapomorphic for Ceratosauria, Spinosauridae, and Paraves, whereas a convex distal profile of the crown is a synapomorphy of Maniraptoriformes (Appendix 6.20 for lateral teeth).

\section{Flutes}

Whole dentition. Flutes are well-known to characterize the dentition of spinosaurids, as they are present on at least some of the mesial and lateral crowns in all known spinosaurid species (e.g., Charig and Milner, 1997; Taquet and Russell, 1998; Sereno et al., 1998; Sues et al., 2002; Dal Sasso et al., 2005; Sales and Schultz, 2017; Figure 18.5). Besides Spinosauridae, the basal theropod Tawa (GR 241; Figure 18.1), juvenile individuals of the coelophysid Coelophysis (Buckley and Currie, 2014), the noasaurid Masiakasaurus (Carrano et al., 2002; Figure 18.4), and an undescribed metriacanthosaurid (ZLJT 0115) appear to be the only non-avian theropods bearing fluted mesial and lateral teeth. All preserved premaxillary, maxillary and dentary teeth seem to be fluted on both sides in Tawa (GR 241). Flutes are restricted to the anterior portion of the maxilla and dentary in juvenile individuals of Coelophysis, as they are present on the labial surface of the crown up to the fifth maxillary and third dentary teeth in RTMP 1984.63.1-1 and to the first maxillary and sixth dentary teeth in NMMNH P-42200 (Buckley and Currie, 2014). In Masiakasaurus, flutes have been observed on the lingual surface of most in situ dentary teeth. They are present in Ldt1 (3 flutes) of FMNH PR 2471 and Rdt4 (5 flutes) and Rdt6 (1 flute) of UA 8680. The latter also seems to have a single flute on the labial surface of the crown, whereas the partially erupted tooth Ldt4 of FMNH PR 2471, of which the apex can only be seen, does not show any fluting.

In Spinosauridae, flutes are present on the lingual surface of all premaxillary teeth of Baryonyx (with the possible exception of the pm1, in which the lingual surface of Lpm1 is badly preserved and hidden by matrix) but absent in Lmx5, the only lateral tooth preserved in situ (NHMUK PV R.9951). Nine out of 14 isolated teeth from the mesial and lateral dentitions (which are impossible to distinguish in Spinosauridae) have flutes, suggesting that at least some lateral teeth were also fluted in Baryonyx. In Suchomimus, flutes can be seen on the labial surface of Lpm2, Lpm3 and mx19 but are absent on both labial and lingual sides of Rmx10 in MNN GDF501. Fluted crowns are also present in the posterior portion of the maxilla of the juvenile specimen MNN G232, and in the central portion of a dentary in MNN G74-1. All preserved maxillary teeth of Irritator (SMNS 58022) are fluted. As for the gigantic snout (MSNM V4047) referred to Spinosaurus, most but not all premaxillary and maxillary teeth are fluted, with flutes being numerous, closely packed and mesiodistally narrow in the largest crowns (i.e., Rpm3, Lmx2, L and Rmx3 and Lmx4). Flutes are essentially restricted to the lingual surface in Baryonyx (Charig and Milner, 1997; n.b., only a single tooth bears several flutes on the lingual side and a single flute on the labial surface; C.H. personal obs.) and present on both sides in most Suchomimus and all Suchosaurus crowns. All preserved teeth of Irritator appear to be fluted on both labial and lingual sides (Sues et al., 2002), and most Spinosaurus teeth have flutes on both sides as well.

Mesial dentition. Flutes restricted to the mesial dentition are present in the ceratosaurid Ceratosaurus (e.g., Madsen and Welles, 2000; UMNH VP 5278; Figure 18.3), the compsognathid Scipionyx (Dal Sasso and Maganuco, 2011; Figure 18.7) and the dromaeosaurid Velociraptor (AMNH 6515; Figure 18.10). While flutes have only been observed on the lingual and labial surface of a single pre- 


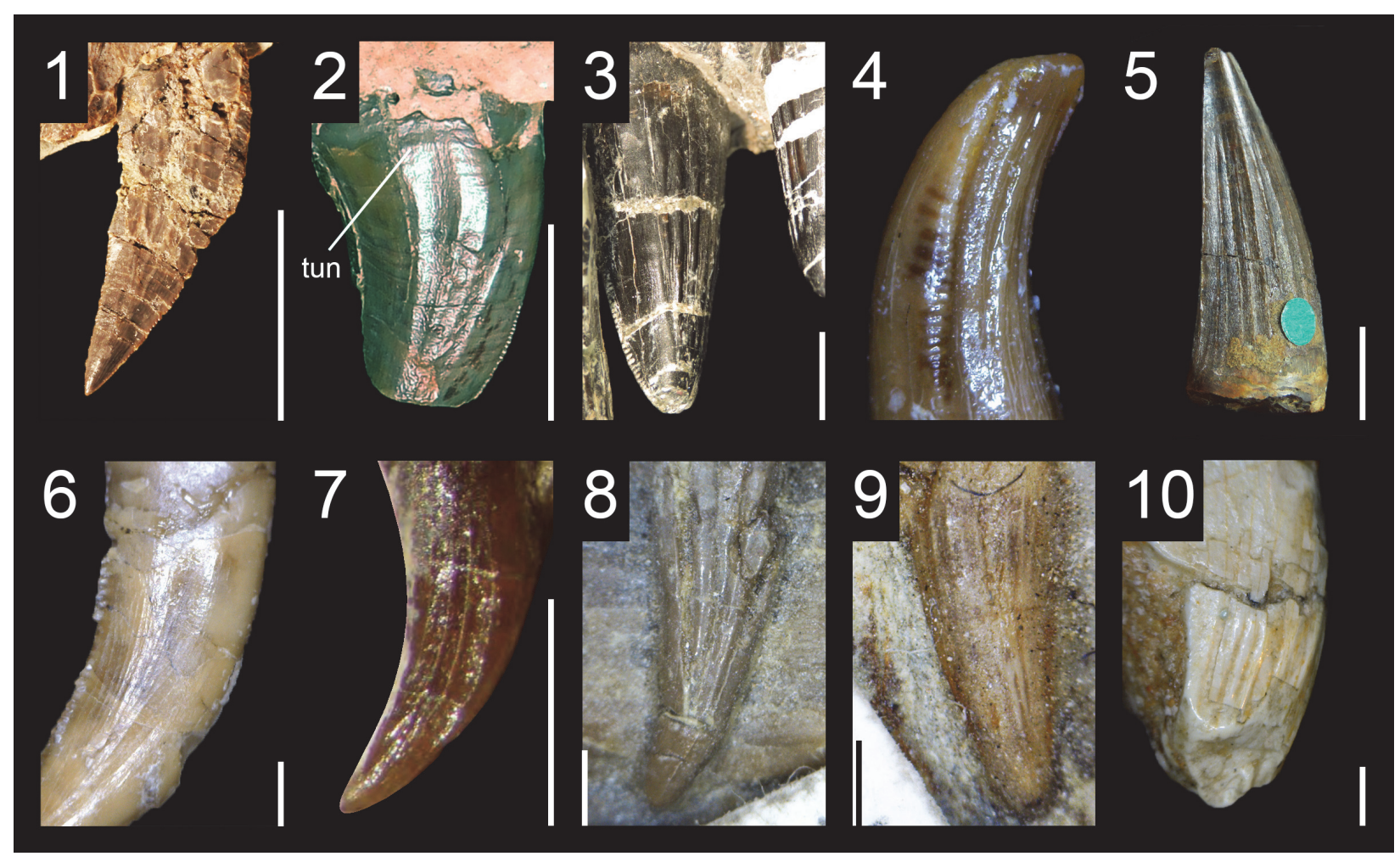

FIGURE 18. Fluted teeth in non-avian Theropoda. 1, Fifth? left maxillary tooth of the basal theropod Tawa hallae (GR 241) in lingual view (courtesy of Sterling Nesbitt); 2, Fourth? left maxillary tooth of the non-averostran neotheropod Sinosaurus triassicus (IVPP V34) in labial view, showing the transverse undulations at the base of the crown; 3, First left and right premaxillary teeth of the ceratosaurid Ceratosaurus nasicornis (UMNH VP 5278) in lingual view; 4, Third right dentary tooth of the noasaurid Masiakasaurus knopfleri (UA 8680) in lingual view; 5, Isolated tooth of the baryonychine Suchosaurus cultridens (= Baryonyx walkeri?; NHMUK PV R.36536) in lingual view; 6, Second right maxillary tooth of the basal pantyrannosaurian Dilong paradoxus (IVPP V14242) in labial view (n.b., an apparent labial concavity delimited by two faint longitudinal ridges results from a lightning effect); 7, Fifth left premaxillary tooth of the compsognathid Scipionyx samniticus (SBA-SA 163760) in lingual view (reprinted with permission from Dal Sasso and Maganuco (2011), courtesy of Cristiano Dal Sasso \& Simone Maganuco, (c) Soprintendenza Archeologia, Belle Arti e Paesaggio per le Province di Caserta e Benevento); 8, Seventh or eight? right maxillary tooth of the dromaeosaurid Microraptor sp. (IVPP 2008.5) in labial view; 9, Fourth left maxillary tooth of the dromaeosaurid Sinornithosaurus millenii (IVPP V12811) in labial view; 10, First right premaxillary tooth of the dromaeosaurid Velociraptor mongoliensis (AMNH 6515) in labial view. Abbreviation: tun, transverse undulation. Scale bars equal $1 \mathrm{~cm}(1-2,4), 1 \mathrm{~mm}(3,5-8)$.

maxillary tooth of Scipionyx (Lpm5) and Velociraptor $(\mathrm{L}$ and $\mathrm{Rpm} 1)$, respectively, they are present on the lingual surface of all premaxillary and at least the first two dentary teeth of Ceratosaurus (UMNH VP 5278).

Lateral dentition. Flutes are visible in the lateral dentition of the non-averostran neotheropod Sinosaurus (IVPP V34; Figure 18.2), the basal pantyrannosaurian Dilong (IVPP V14242; Figure 18.6), and the dromaeosaurids Austroraptor (Novas et al., 2009), Microraptor (IVPP 2008.5; Figure 18.8), and Sinornithosaurus (IVPP V12811; Figure 18.9). They are also present in Zapsalis (Cope, 1876a; Larson and Currie, 2013) and Paronychodon (e.g., Cope, 1876b; Baszio, 1997; Hwang, 2005; Sankey, 2008), which is interpreted as being a tooth mor- photype of Richardoestesia gilmorei by Longrich (2008). Flutes have been described to be present on the apical part of the crown in Mononykus (Perle et al., 1994) but they represent faint longitudinal grooves rather than genuine flutes. If the distribution of flutes is unknown in Austroraptor, flutes have only been observed in a few lateral teeth in Dilong (Rmx1, Rmx2, Rmx4, Ldt5) and Sinornithosaurus ( $R m \times 2, R m \times 4)$, and in a single tooth in Sinosaurus (IVPP V34) and Microraptor (IVPP 2008.5). Most of the teeth are, however, unpreserved or obscured in these two specimens.

Remarks and synapomorphy. Fluted teeth seem to be an ontogenetic feature in Coelophysis as only juvenile-sized skulls display this feature (Buckley and Currie, 2014). According to Buckley and Currie 
(2014), such ontogenetic variation may indicate different diets between juvenile and mature individuals, with young Coelophysis primarily eating arthropods and fish. This ontogenetic pattern can also be observed in the basal pantyrannosaurian Dilong as the crowns of IVPP V14243 are not fluted whereas the specimen IVPP V14242, which represents an earlier ontogenetic stage than IVPP V14243 (Xu et al., 2004), clearly bears several fluted teeth.

The number of flutes on the labial or lingual surface of the crown ranges from one to eight in juvenile Coelophysis (Buckley, 2009, figure 4.3, 4.4 and 4.5), four to seven in the mesial dentition of Ceratosaurus (4-6 flutes in L/Rpm1-3 and 4-5 in Ldt1-2 of UMNH VP 5278; Figure 18.3), two to five in the mesial crowns of Masiakasaurus (3-4 in average; UA 8680, FMNH PR 2182, 2471, 2696; Figure 18.4), and three in a single maxillary crown of Sinosaurus (IVPP V34; Figure 18.2). In spinosaurids, we counted four to eight flutes in Baryonyx (6-7 in average; NHMUK PV R.9951), ten to 12 in Suchosaurus cultridens (NHMUK PV R.3308, R.4702, and R.36536; Figure 18.5), two to 10 in Suchomimus (6-7 in average; UC G26-5, G34-7, UC G67-1), and five to 10 in Irritator (7-8 in average; SMNS 58022). The highest number of flutes in theropods is seen in the unserrated crowns of some spinosaurines, with up to 17 flutes in 'Sinopliosaurus' (IVPP V4793.2) and Siamosaurus (TF 2043), and 20 flutes in the largest maxillary crowns of Spinosaurus (Lmx2 of MSNM V4047). In averostrans, there are four to six, possibly more in the undescribed metriacanthosaurid ZLJ 0115, 12 to 13 flutes in Dilong (Rmx2 and 4 of IVPP 14242; Figure 18.6), two or three in Scipionyx (Dal Sasso and Maganuco, 2011, figure 45; Figure 18.7), four or five in Microraptor (IVPP 2008.5; Figure 18.8), eight or nine in Sinornithosaurus (Rmx4 of IVPP V12811; Figure 18.9), and around three to six flutes in the first premaxillary teeth of Velociraptor (AMNH 6515; Figure 18.10). Fluted mesial and lateral teeth are a synapomorphy of Spinosauridae (Sereno et al., 1998), whereas fluted lateral teeth are here considered as synapomorphic for Microraptorinae (Appendix 6.21).

Functional morphology. The presence of flutes, usually on conical teeth, is common in piscivorous tetrapods such as crocodiles (Longrich, 2008), mosasaurs, plesiosaurs (Massare, 1987) and pterosaurs (e.g., Kellner and Tomida, 2000; Andres et al., 2010). Longitudinal ridges bounding flutes are present in a large array of tooth morphologies associated with different diets in marine reptiles
(Massare, 1987). Plesiosauroid teeth with long slender conidont crowns and flutes delimited by low longitudinal ridges suggest that they were used to pierce soft prey, whereas the straight and robust cones with flutes delimited by prominent ridges were used for grasping prey with a hard exterior in ichthyosaurs. Likewise, the longitudinal and sharp ridge bounding each flute of large pliosaurids is probably a cutting edge used for tearing fleshy prey (Massare, 1987). Therefore, the presence of flutes on the crown may have a broad and general function in animals with disparate tooth morphologies and teeth adapted to different diets. Flutes most likely have some piercing and gripping function, allowing the sharp ridges to pierce the skin and broadening the flesh as the tooth penetrates the prey body, and keeping slippery prey in the mouth (Sues et al., 2002). Fluted crowns, which appear to be more difficult to pull out compared to non-fluted crowns given that the contact area between the meat and the teeth is larger, may have prevented the struggling prey from rotating and also helped to resist its movements (Kouzuma S. and Kamiya M., personal comm., 2017).

\section{Transverse undulations}

Although thought to be a possible tetanuran synapomorphy (Brusatte et al., 2007), transverse undulations (Figure 19) are present in the crowns of many non-avian theropods, from basal to derived forms. These are present in: the basalmost saurischians Ischisaurus (MACN 18.060), Eodromaeus (PVSJ 561) and Sanjuansaurus (PVSJ 605; Figure 19.1); the basal theropods Dracoraptor (NMW 2015.5G) and Dracovenator (BP/1/5243); the basal ceratosaur Berberosaurus (MNHN To 369); the ceratosaurids Ceratosaurus (USNM 4735) and Genyodectes (MLP 26-39; Figure 19.2); the noasaurid Masiakasaurus (FMNH PR.2221, 2476; Figure 19.3), and the abelisaurids Aucasaurus (MCF-PVPH 236; Figure 19.4) and Majungasaurus (FMNH PR.2278). As noted by Brusatte et al. (2007), transverse undulations are widespread among basal tetanurans and have been observed in: the megalosauroid Monolophosaurus (IVPP V84019; Brusatte et al., 2010a; Figure 19.5); the piatnitzkysaurids Marshosaurus (DMNS 3718; Figure 19.6) and Piatnitzkysaurus (PVL 4073; MACN $\mathrm{CH}$ 895); the megalosaurids Duriavenator (NHMUK PV R.332) and Megalosaurus (NHMUK PV R.8303; OUMNH J13505); the spinosaurid Baryonyx (NHMUK PV R.9951; ML 1190); the allosauroids Acrocanthosaurus (NCSM 14345), Allosaurus (AMNH 851; NHFO 455; UMNH VP5841; 


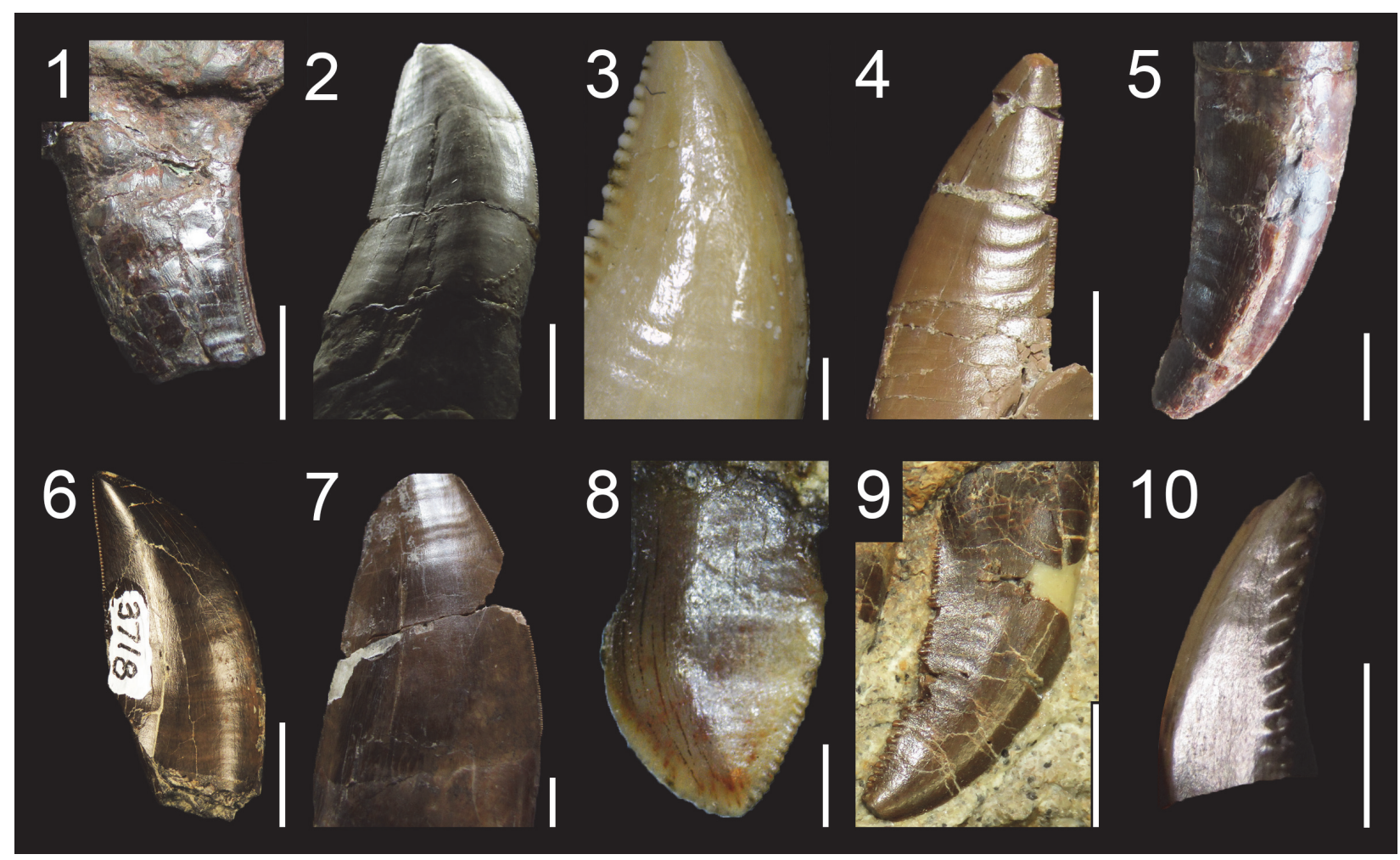

FIGURE 19. Transverse undulations in the teeth of non-avian Saurischia. 1, Fifth? left maxillary tooth of the herrerasaurid Sanjuansaurus gordilloi (PVSJ 605) in labial view; 2,Fifth right dentary tooth of the ceratosaurid Genyodectes serus (MLP 26-39) in basolabial view; 3, Isolated lateral tooth of the noasaurid Masiakasaurus knopfleri (FMNH PR 2221) in labial view; 4, Isolated lateral tooth of the abelisaurid Aucasaurus garridoi (MCF-PVPH-236) in labial? view; 5, Fourth right premaxillary tooth of the megalosauroid Monolophosaurus jiangi (IVPP 84019) in labial view; 6, Isolated lateral tooth of the piatnitzkysaurid Marshosaurus bicentesimus (DMNS 3718) in lingual view; 7, Isolated lateral tooth of the carcharodontosaurid Giganotosaurus carolinii (MUCPv-CH-1) in labial view; 8, Fourteenth left maxillary tooth of the therizinosaur Falcarius utahensis (UMNH VP 14545) in labial view; 9, Fifth right maxillary tooth of the dromaeosaurid Atrociraptor marshalli (TMP 1995.166.01) in labial view; 10, Isolated tooth of the troodontid Troodon formosus (DMNH 22337) in labiodistal view. Scale bars $=1 \mathrm{~cm}(1-2,4-7), 5 \mathrm{~mm}(9-10), 1 \mathrm{~mm}(3,8)$.

Figure 20.7), Concavenator (Cuesta et al., 2018), Neovenator (MIWG 6348), Giganotosaurus (MUCPV-CH-1; Figure 19.7), and Sinraptor (IVPP 10600); the tyrannosauroids Alioramus (MPC-D 100-1844; Brusatte et al., 2012), Dilong (IVPP V11979), Guanlong (IVPP V14532), Gorgosaurus (USNM 12814), Teratophoneus (UMNH VP 16690; Figure 20.10) and Tyrannosaurus (FMNH PR.2081); and the basal coelurosaurs Ornitholestes (AMNH 619) and Zuolong (Choiniere et al., 2010a). Among neocoelurosaurs, they are present in: the therizinosaurs Falcarius (UMNH VP 14545; Figure 19.8) and Segnosaurus (MPC-D 100-80); the dromaeosaurids Atrociraptor (TMP 1995.166.01; Figure 19.9), Deinonychus (YPM 5232), Dromaeosaurus (Smith, 2005; AMNH 5356), Saurornitholestes (TMP 1988.121.39, 1994.12.844), Utahraptor (BYU 1249) and an inde- terminate velociraptorine (DMNH unnumbered); and the troodontid Troodon (DMNH 22337; Figure 19.10).

Remarks and synapomorphy. Transverse undulations are not restricted to the crown as they have also been observed on the root of various averostrans such as Aucasaurus (MCF-PVPH 236), Allosaurus (UMNH VP 1251, 5841), Baryonyx (NHMUK PV R.9951), Neovenator (MIVG 6348), Saurornitholestes (TMP 1988.121.39), Zuolong (Choiniere et al., 2010a) and an immature specimen of Daspletosaurus (TMP 1994.143.1). Transverse undulations may be genuinely absent in the dentition of Alvarezsauroidea, Compsognathidae, Halszkaraptorinae, Microraptorinae, Ornithomimosauria, Oviraptorosauria, Scansoriopterygidae and Unenlagiinae, given that they are not observed in these clades. Likewise, enamel undu- 


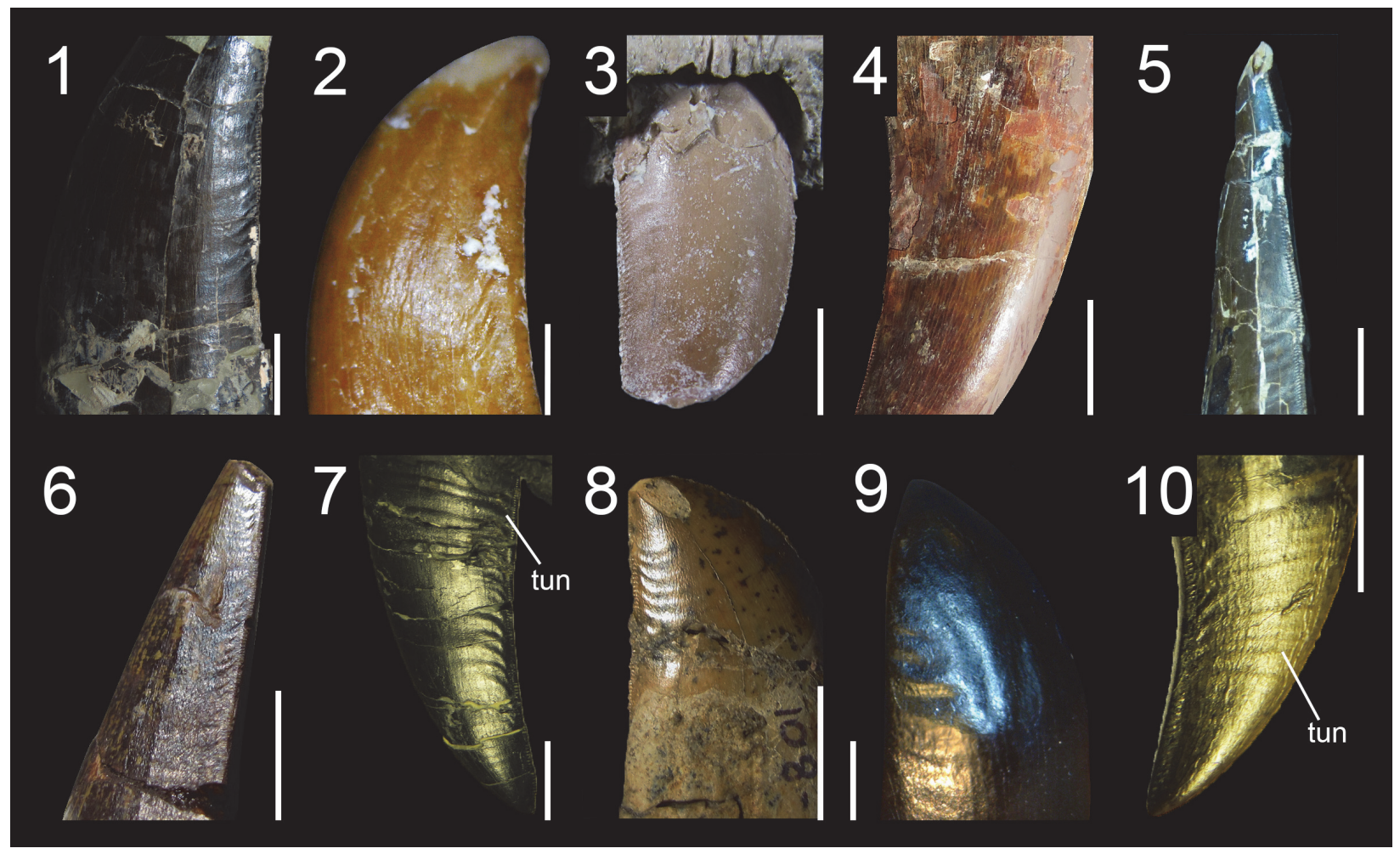

FIGURE 20. Marginal undulations in the teeth of non-avian Theropoda. 1, Fifth left maxillary tooth of the ceratosaurid Ceratosaurus nasicornis (UMNH VP 5278; reversed) in lingual view; 2, Second left maxillary tooth of the noasaurid Masiakasaurus knopfleri (FMNH PR.2696) in labiodistal view; 3, Second left maxillary tooth of the abelisaurid Majungasaurus crenatissimus (FMNH PR 2100) in basolingual view; 4,Second right maxillary crown of the megalosauroid Monolophosaurus jiangi (IVPP 84019) in labial view; 5, Isolated lateral tooth of the piatnitzkysaurid Piatnitzkysaurus floresi (MACN 895) in linguodistal view; 6, Isolated lateral tooth of the spinosaurid Suchomimus tenerensis (MNN G51) in labiodistal? view; 7, Sixth left maxillary tooth of the allosaurid Allosaurus sp. (UMNH VP 9168) in labial view and showing both the marginal and transverse undulations on the crown; 8, Distalmost isolated tooth of the carcharodontosaurid Mapusaurus roseae (MCF-PVPH-108.103) in basolabial view; 9, Isolated mesial tooth of the basal pantyrannosaurian Aviatyrannis jurassica (MG27801 D90) in labial view; 10, Isolated lateral tooth of the pantyrannosaurian Teratophoneus curriei (UMNH VP 16690) in basolabial view, showing both the marginal and transverse undulations. Abbreviation: tun, transverse undulation. Scale bars equal $1 \mathrm{~cm}(1,3-8), 5 \mathrm{~mm}(10), 1 \mathrm{~mm}(2,9)$.

lations (i.e., transverse and marginal undulations) have not been observed in the teeth of any coelophysoids, and these theropods may also lack these dental features. Due to the wide distribution of these crown ornamentations in non-avian theropods, enamel undulations have to be used in concert with other dental features in order to assign teeth to more restricted theropod clades. Nevertheless, the presence of numerous and closely packed transverse undulations seems to be restricted to the crowns of non-neocoelurosaur avetheropods such as Allosauroidea, Ceratosauridae, Megalosauridae, Piatnitzkysauridae and Tyrannosauroidea, as well as some dromaeosaurids like Deinonychus and Saurornitholestes (C.H. personal obs.). Transverse undulations are here considered to be synapomorphic for Avetheropoda (Appendix 6.22).

Functional morphology. Transverse undulations may have served to minimize suction when the tooth was pulled out of the flesh (Currie and Azuma, 2006), to help strengthen the crown during feeding (Brusatte, 2012), or may simply be a byproduct of growth (Brusatte et al., 2007). Transverse undulations in large meat-eating theropods are not necessarily homologous to those in taxa such as Troodon and Dromaeosaurus, and may differ in their development and origin. Transverse undulations are also present in many other tetrapods, including pliosaurids (Zverkov et al., 2018), mosasaurids (Buffetaut and Bardet, 2012), metriorhynchid crocodylomorphs (De Andrade et al., 
2010) and rauisuchian crurotarsans (Brusatte et al., 2009a).

\section{Marginal undulations}

Short and marginal undulations close to the carinae are a well-known feature of carcharodontosaurid teeth (Sereno et al., 1996; Coria and Currie, 2006) as they appear on the crowns of Carcharodontosaurus (SGM Din-1; UC PV6; MNN IGU6), Concavenator (Cuesta et al., 2018), Giganotosaurus (MUCPv-CH-1), Mapusaurus (MCF-PVPH 108; Figure 20.8) and Tyrannotitan (Canale et al., 2015). Marginal undulations (Figure 20) have also been reported in the abelisaurid Skorpiovenator (Canale et al., 2009) and are present in a large range of non-neocoelurosaur avetheropods: the ceratosaurs Abelisaurus (MC 5, MC 687), Ceratosaurus (USNM 4735; Figure 20.1), Chenanisaurus (Longrich et al., 2017), Majungasaurus (FMNH 2100; Figure 20.3) and Masiakasaurus (FMNH PR.2182; Figure 20.2); the megalosauroid Monolophosaurus (IVPP V84019; Figure 20.4); the piatnitzkysaurids Marshosaurus (DMNS 3718) and Piatnitzkysaurus (PVL 4073; MACN 895; Figure 20.5); the megalosaurids Afrovenator (UC UBA1), Megalosaurus (NHMUK PV R.234; OUMNH J.23014), and Torvosaurus (ML 500; Hendrickx et al., 2015c); the spinosaurids Baryonyx (NHMUK PV R.9951), Irritator (Sues et al., 2002; Figure 15.3), Suchomimus (MNN G35-9; Figure 20.6) and an indeterminate spinosaurine from Brazil (Medeiros, 2006); the pantyrannosaurians Aviatyrannis (MG 27801 D90; Figure 20.9), Daspletosaurus (NHMUK PV R.4863), Lythronax (UMNH VP 20200), Teratophoneus (UMNH VP 16690; Figure 20.10), and Tyrannosaurus (Brusatte et al., 2007); and many non-carcharodontosaurine allosauroids such as Acrocanthosaurus (NCSM 14345), Allosaurus (e.g., USNM 8335; UMNH VP 5837, 6113, 7436; Figure 20.7), Fukuiraptor (FPDM 9712204; Azuma and Currie, 2000, figure 4 bottom right), Neovenator (MIWG 6348) and Sinraptor (IVPP V10600).

Remarks and synapomorphy. In most theropods, marginal undulations usually extend mesiodistally on the crown, and are typically elongated and parabolic, with the part adjacent to the carina curving apically. However, marginal undulations of some theropods can be short, broad, and mesiodistally straight, as seen adjacent to the mesial carina of Afrovenator (MNN UBA1), Ceratosaurus (USNM 4735; Hendrickx et al., 2015c, figure A5.L), Masiakasaurus (FMNH PR.2182) and Tyrannosaurus (TMP 1981.006.01). They can also be strongly diagonally-oriented, as observed in some teeth of Irritator (Sues et al., 2002, figure 5), Masiakasaurus (FMNH PR.2696; Figure 20.2), Monolophosaurus (IVPP V84019; Figure 20.4), Megalosaurus (OUMNH J.23014), Suchomimus (MNN G51; Figure 20.6), and an indeterminate spinosaurid (Medeiros, 2006). Numerous and extremely pronounced marginal undulations on both mesial and distal sides of the crown have only been identified in Carcharodontosaurus saharicus and seem to be autapomorphic to this taxon among theropods (Brusatte and Sereno, 2007). The marginal undulations of other carcharodontosaurids such as Acrocanthosaurus, Carcharodontosaurus iguidensis, Concavenator, Giganotosaurus, Mapusaurus and Tyrannotitan are present but not as pronounced and numerous as those visible in the teeth of the neotype of Carcharodontosaurus saharicus (Brusatte and Sereno, 2007; C.H. personal obs.). Some Allosaurus lateral crowns also show numerous and particularly pronounced marginal undulations, yet they are mesiodistally shorter and restricted to the distal carina of the crown (Figure 20.7).

Marginal undulation is a more widespread feature than previously thought in non-avian theropods, and the presence of marginal undulations is here considered to be a synapomorphy of Tetanurae (Appendix 6.23). Yet, we cannot entirely dismiss the hypothesis that this feature is convergently present in several clades of theropods as it might have a particular functional role that converged after the split of the various theropod clades possessing it. Interestingly, marginal undulations are present only in large-sized theropods with the exception of Masiakasaurus and Aviatyrannis.

\section{Interdenticular sulci}

Elongated interdenticular sulci. Thought to be restricted to Allosauroidea and Tyrannosauroidea ('Carnosauria' sensu Gauthier, 1986; Rauhut and Kriwet, 1994) or to represent an abelisaurid synapomorphy (Smith, 2007), interdenticular sulci (Figure 21), in fact, occur in many other theropod clades. Strongly developed and elongated sulci have been observed in the abelisaurids Chenanisaurus (Longrich et al., 2017), Kryptops (MNN GAD1-1), and Majungasaurus (FMNH PR.2100, 2278; Figure 21.2) and the non-spinosaurid megalosauroids Megalosaurus (OUMNH J13506; Figure 21.4), Piatnitzkysaurus (PVL 4073; Figure 21.3) and Torvosaurus (Hendrickx and Mateus, 2014b). In avetheropods, they are also present in the allo- 


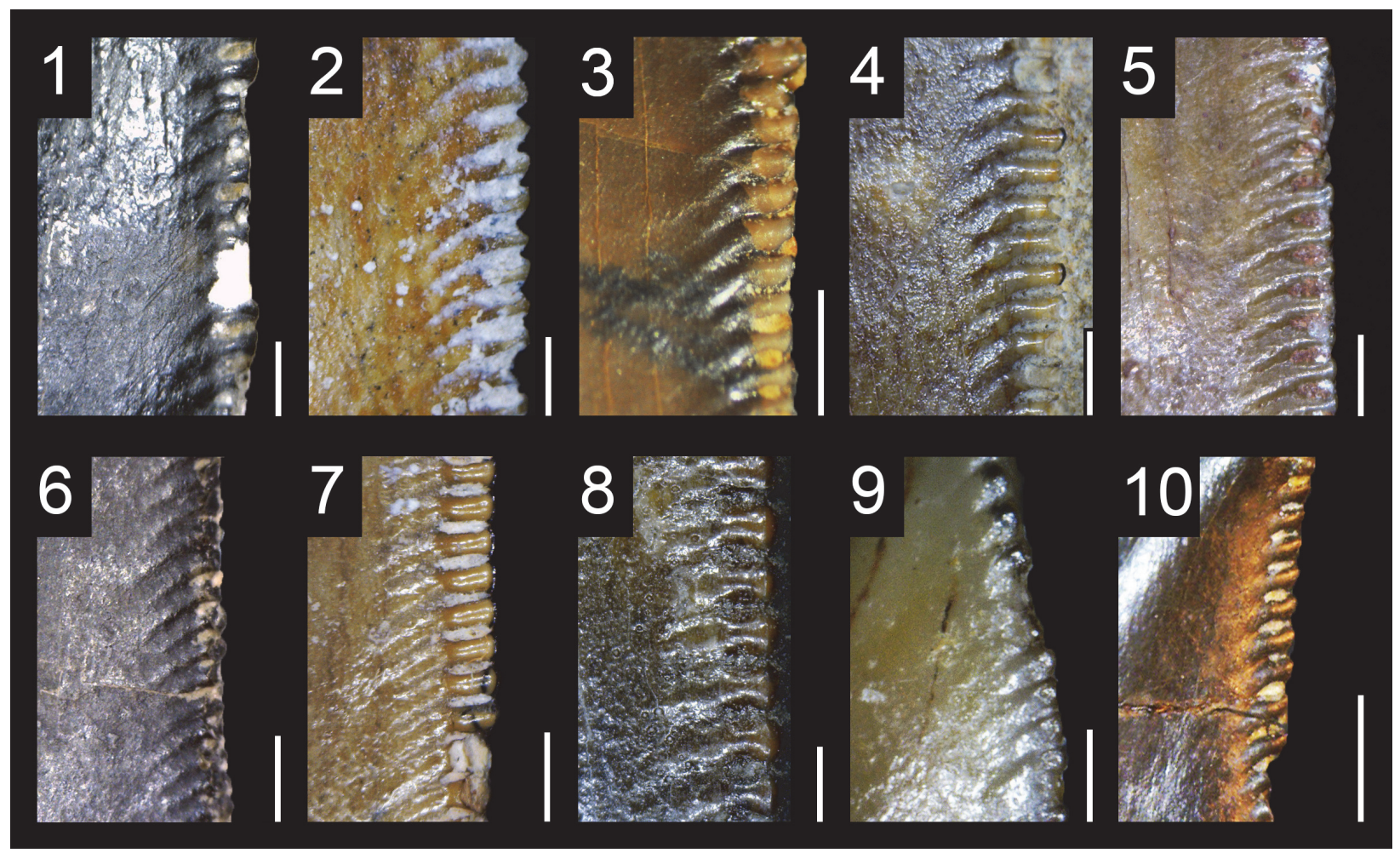

FIGURE 21. Well-developed interdenticular sulci in non-avian Theropoda. 1, Distal carina of the eighth left maxillary tooth of the ceratosaurid Ceratosaurus nasicornis (UMNH VP 5278; image upside down) in lateral view; 2, Distal carina of the sixth right maxillary tooth of the abelisaurid Majungasaurus crenatissimus (FMNH PR.2278; image upside down) in lateral view; 3, Distal carina of an isolated lateral tooth of the piatnitzkysaurid Piatnitzkysaurus floresi (PVL 4073) in lateral view; 4, Distal carina of an isolated lateral tooth of the megalosaurid Megalosaurus bucklandi (NHMUK PV R.234) in labial view; 5, Distal carina of an isolated lateral tooth of the metriacanthosaurid Sinraptor dongi (IVPP V10600) in lateral view; 6, Distal carina of an isolated tooth of the allosaurid Allosaurus sp. (UMNH VP 6177 ) in lateral view, also showing the transverse undulations at the crown base; 7 , Distal carina of an isolated lateral tooth of the carcharodontosaurid Giganotosaurus carolinii (MUCPv CH1 L2) in lateral view; 8, Distal carina of the fifth right maxillary tooth of the tyrannosaurid Tyrannosaurus rex (FMNH PR.2081; image upside down) in lateral view; 9, Distal carina of a right mid-dentary tooth of the basal therizinosaur Falcarius utahensis (UMNH VP 14528) in labial view; 10, Distal carina of the last? right dentary tooth of the dromaeosaurid Deinonychus anthirrhopus (YPM 5232) in labial view. Scale bars equal $1 \mathrm{~mm}$.

sauroids Allosaurus (UMNH VP 6499, 10393; Figure 21.6), Fukuiraptor (Azuma and Currie, 2000), Giganotosaurus (MUCPv-CH-1; Figure 21.7), Mapusaurus (MCF-PVPH-108) and Sinraptor (IVPP 10600; Figure 21.5), and the tyrannosaurids Albertosaurus (TMP 1999.50.40), Daspletosaurus (TMP 1994.143.1) and Tyrannosaurus (FMNH PR.2081; Figure 21.8). In non-paravian maniraptoriforms, they have only been observed in the basal therizinosaur Falcarius (UMNH VP 14528; Figure 21.9).

Short interdenticular sulci. Poorly-developed interdenticular sulci are widespread among nonneocoelurosaur theropods as they have been noticed in the basal theropod Tawa (GR 241), and almost all allosauroids, ceratosaurids (Figure 21.1), megalosaurids, piatnitzkysaurids (including
Condorraptor; contra Rauhut, 2005) and tyrannosauroids (e.g., Currie et al., 1990; Azuma and Currie, 2000; Fanti and Therrien, 2007; Hendrickx and Mateus, 2014a; Hendrickx et al., 2015c; C.H. personal obs.). In paravians, they have also been observed in the microraptorine specimen IVPP V13476 (Xu and Li, 2016), the eudromaeosaurians Deinonychus (YPM 5232; Figure 21.10), Saurornitholestes (Currie et al., 1990) and Dromaeosaurus (AMNH 5356; Currie et al., 1990; Larson, 2008), as well as some troodontids (Currie and Dong, 2001b; Sankey, 2008, figure 3.13 and 3.21) such as Troodon (NHMUK PV R.12568).

Remarks and synapomorphy. Interdenticular sulci have not been seen in herrerasaurids, coelophysoids, dilophosaurids, noasaurids, spinosaurids, several abelisaurids such as Rugops (MNN 
IGU1), Aucasaurus (MCF-PVPH 236) and Skorpiovenator (MMCN-PV 48), the megalosauroid Monolophosaurus (IVPP 84019), and the large majority of non-paravian neocoelurosaurs. Nonetheless, given the widespread distribution of this feature in faunivorous theropods, we suspect the presence of interdenticular sulci in these taxa, especially in herrerasaurids, dilophosaurids and Monolophosaurus. Interdenticular sulci are, for now, considered to be synapomorphic for Abelisauridae, Allosauroidea, Ceratosauridae, Piatnitzkysauridae and Tyrannosauroidea (Appendix 6.24 for lateral teeth). Likewise, the presence of strongly-developed interdenticular sulci, which seem to be restricted to non-coelurosaur averostrans and basal therizinosaurs (Young et al., 2019), is a possible apomorphic dental character of the clades Abelisauridae, Allosauroidea, Giganotosaurini and Tyrannosauridae.

Functional morphology. Interdenticular sulci have been noticed in several theropods by many authors (Currie et al., 1990; Abler, 1992; Buscalioni et al., 1996; Smith, 2007; Benson, 2009), but none provided functional implications for these structures. Interdenticular sulci may play several roles such as helping the entry of venom in a possible venomous theropod, hosting septic bacteria for an infectious bite, distributing stresses from the base of the denticle, or preventing suction when the crown was pulled out of the flesh. The first hypothesis is supported by the presence of interdenticular sulci on the crown of the venomous Komodo dragon (D'Amore and Blumenschine, 2009), representing a rare example of an extant animal showing these sulci. The teeth of the Komodo dragon do not have any venom-delivering systems, unlike the hollow teeth of snakes or the apicobasally long grooves on the crowns of helodermatid lizards (Fry et al., 2009). Instead, its crowns are smooth and lack any dental features besides short interdenticular sulci and plicidentine (i.e., the radial infolding of the dentine layer of the basal portion of the crown, present in some fish, amphibians, and varanoids; e.g., Peyer, 1968; Kearney et al., 2006; Maxwell et al., 2011), so that the venom seems to enter via deep wounds when lacerating the prey items (Fry et al., 2009). Therefore, interdenticular sulci of Varanus komodoensis and theropods may help venom to be administrated during biting.

The second hypothesis was proposed by Abler $(1997,1999)$ for the deep interdenticular space ('cella' sensu Abler, 1992, 1999) present in between tyrannosaurid denticles. Abler (1992, 1999) suggested that these interdenticular spaces would trap grease and food debris that functioned as receptacles for septic bacteria, becoming the source of a lethal infection when biting. Pathogenic bacteria have also been reported in the saliva of the Komodo dragon (Auffenberg, 1981; Montgomery et al., 2002), and interdenticular sulci may help the proliferation and entering of these toxic bacteria within the prey. Fry et al. (2009), however, note that no compelling evidence supports the presence of pathogenic bacteria in the saliva of Varanus komodoensis, a hypothesis supported by Goldstein et al. (2013) based on captive Komodo dragons. Yet, Tehrani et al. (2008) and Vikrant and Verma (2014) have reported septic effects of varanid bites, and pathogenic bacteria might, therefore, be present and possibly hosted within interdenticular sulci of the crowns of wild varanids.

Interdenticular sulci may also serve as stressdistributing structures as they re-orient the stresses at the base of the denticle towards the middle of the crown, thus preserving the integrity of the denticle under high-stress regimes. Contrasting with the disparate morphology of hooked denticles among different theropod clades, interdenticular sulci are similar, possibly homologous, in Ceratosauridae, Megalosauroidea, Allosauroidea, Tyrannosauroidea, Therizinosauria and Paraves. Given that these theropods were faunivorous, herbivorous or omnivorous, this condition favors the idea that interdenticular sulci are stress-dissipation or suction reduction structures, rather than specialized infectious or venomous delivery systems. It is, nonetheless, possible that interdenticular sulci had a combination of several or all of these functions in some theropods.

\section{Longitudinal ridges}

Mesial dentition. A longitudinal ridge centrally positioned on the lingual surface of the crown and delimited by two concave surfaces is typical of tyrannosauroid mesial teeth (Carr and Williamson, 2004). This feature has been observed in the pantyrannosaurians Albertosaurus (Carr and Williamson, 2004), Appalachiosaurus (Carr et al., 2005), Daspletosaurus (Lehman and Carpenter, 1990; TMP 1994.143.01), Gorgosaurus (Cillari, 2010), Nanotyrannus (Carr and Williamson, 2004; n.b., the small-sized Lmx1 of LACM 28471 displaying a $\mathrm{U}$-shaped cross-section and a medial ridge is here considered to be a premaxillary tooth; Molnar, 1978; contra Carr and Williamson, 2004), Raptorex (Sereno et al., 2009; LH PV18; Figure 22.2), Timurlengia (Averianov and Sues, 2012), Tyrannosaurus (Smith, 2005; MOR 1125), Xiongguanlong (Li et al., 
2010; Figure 11.4) and an indeterminate tyrannosauroid from the Cenomanian of Utah (Zanno et al., 2019). A discrete ridge also occurs on the lingual side of the mesial premaxillary teeth in the basal tyrannosauroids Dilong (IVPP V11979, V14243) and Proceratosaurus (Lpm2; NHMUK PV R.4860). In Proceratosaurus, the median ridge seems, however, to be a byproduct of the numerous basal striations extending on the basal half or basal two-thirds of the lingual surface of the crowns. This median ridge is absent in the proceratosaurid Guanlong (IVPP V14531; contra Sereno et al., 2009) as well as some basal pantyrannosaurians such as Aviatyrannis (MG 2781 D90 and D172) and Eotyrannus (MIWG 1997.550). Outside Tyrannosauroidea, one or several longitudinal ridges can be seen on the lingual surfaces of some premaxillary teeth of the basal coelurosaur Fukuiraptor (Azuma et al., 2016), and the microraptorines Sinornithosaurus (IVPP V12811) and some specimens of Microraptor (IVPP V13320, 2008.5). Likewise, a mesiodistally wide and centrally positioned convexity delimited by two concavities adjacent to the carinae on the lingual surface of some premaxillary crowns of Allosaurus (YPM-PU 14554) can also be considered as a longitudinal ridge.

Lateral dentition. Two longitudinal ridges delimiting the lingual depression are observable on the lingual surface of some lateral teeth of Orkoraptor (Novas et al., 2008; Figure 22.1). One, two or several longitudinal ridges delimiting grooves of irregular width and orientation are present on the crowns of the basal therizinosaur Falcarius (Zanno, 2010a) and many paravians. In Falcarius, a single centrally positioned longitudinal ridge running along the whole crown height can be observed on the lingual surface of the first maxillary tooth of UMNH VP 14545 and several mid-dentary teeth (Zanno, 2010b, figure 2L; Button et al. (2017), supplemental figure S2B). In dromaeosaurids, they have been noticed in Bambiraptor (AMNH 30556; Figure 22.3), Buitreraptor (Gianechini et al., 2011b; MPCA 245), Changyuraptor (Han et al., 2014, figure 3b), Linheraptor (IVPP V16923), Microraptor (IVPP 2008.5), Richardoestesia (Longrich, 2008; Sankey, 2008), Saurornitholestes (Baszio, 1997; Sankey, 2008), Velociraptor (AMNH 6515), and Zapsalis (Larson, 2008; Longrich, 2008; Larson and Currie, 2013), as well as Acheroraptor, in which they are numerous and particularly well-visible (Evans et al., 2013; Figure 22.4). They are also present in some troodontids such as cf. Pectinodon (Sankey, 2008; Larson and Currie, 2013), Troodon (Currie,

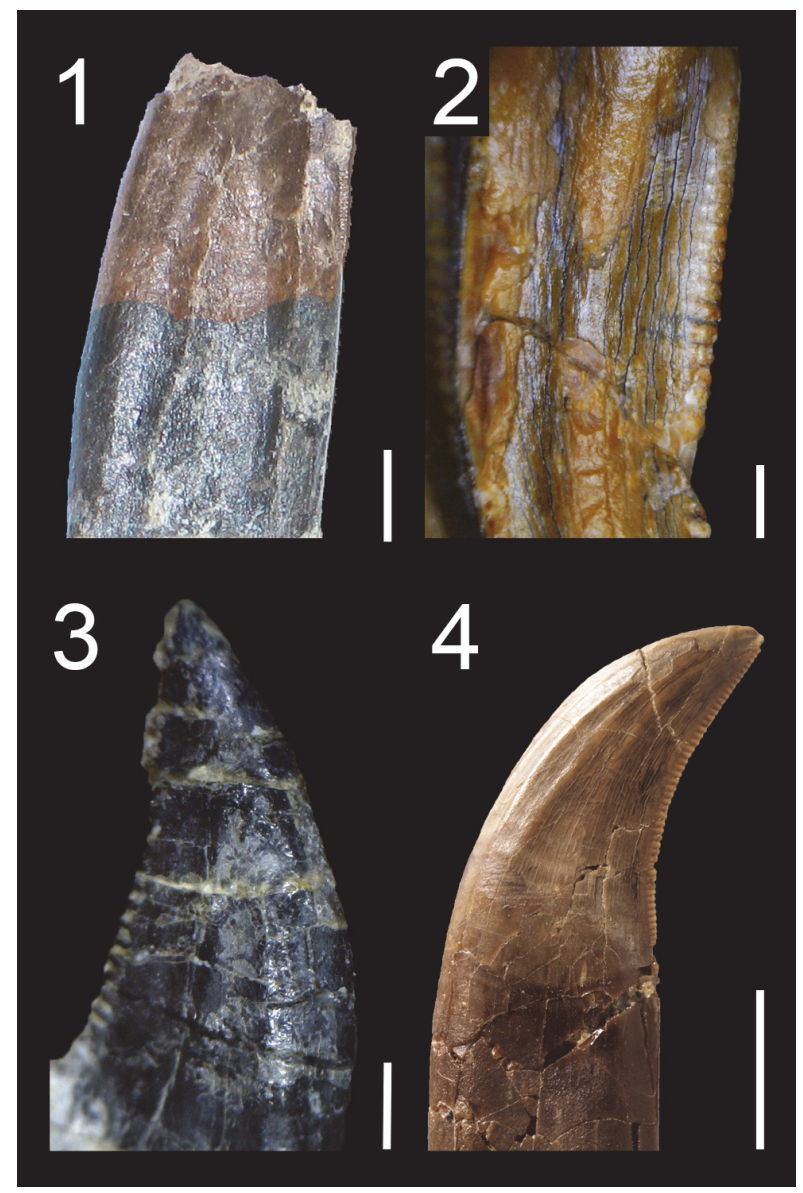

FIGURE 22. Longitudinal ridges in the teeth of nonavian Theropoda. 1, Isolated tooth of the megaraptoran Orkoraptor burkei (MPM-Pv 3458) in lateral view (courtesy of M. Ezcurra); 2, Fourth right premaxillary tooth of the pantyrannosaurian Raptorex kriegsteini (LH PV18) in mesiolabial view; 3, Fifth left maxillary tooth of the dromaeosaurid Bambiraptor feinbergi (AMNH 30556) in labial view; 4, Second? maxillary tooth of the dromaeosaurid Acheroraptor temertyorum (ROM 63777) in labial view (courtesy of D. Larson). Scale bars equal $1 \mathrm{~cm} \mathrm{(1,}$ 4 ) and $1 \mathrm{~mm}(2-3)$.

1987, figure 5S; Sankey, 2008) and the indeterminate paravians and tooth-based taxa Euronychodon (Antunes and Sigogneau-Russell, 1991) and Paronychodon (Currie et al., 1990; Zinke and Rauhut, 1994; Baszio, 1997; Larson, 2008; Sankey, 2008). In the ziphodont teeth of troodontids, a prominent median ridge appears to be present on the lingual surface of some maxillary crowns in Xixiasaurus (Lü et al., 2010, figure 3A1) and is visible on the labial surface of the some maxillary teeth in Byronosaurus (Makovicky et al., 2003; Figure 6.3). Faint longitudinal ridges delimiting shallow and narrow grooves were reported by Choiniere et al. 
(2014b) on the largest crowns of the lateral dentition in the basal alvarezsauroid Haplocheirus, yet we could not observe this feature.

Remarks and synapomorphy. The presence of one or several ridges extending diagonally or parallel to the crown margins on the labial and/or lingual surface of the crown is a possible synapomorphy of Paravians. Among tyrannosauroids, a median longitudinal ridge on the lingual surface of mesial teeth is a synapomorphy of a clade encompassing Timurlengia and more derived tyrannosauroids (Brusatte et al., 2016; Appendix 6.25 for mesial teeth). This feature seems to be absent in more basal tyrannosauroids such as Aviatyrannis, Eotyrannus, Guanlong and Proceratosaurus, with the exception of Dilong which is here considered as autapomorphic. The longitudinal ridges in dromaeosaurids seem to be a genuine diagnostic feature (Evans et al., 2013) that could be apomorphic for some dromaeosaurid clades.

Functional morphology. Prominent ridges delimiting deep grooves dorsal to the plicidentine are present on the crown of the possibly venomous Varanus (Megalania) priscus (Fry et al., 2009, figure 3), which also possesses a ziphodont dentition. These ridges are similar to those observed in Bambiraptor, Buitreraptor and Velociraptor which delimit the labial depression on the crown. These structures may have helped venom to enter in the prey flesh. Other authors proposed that the labial and lingual depressions in the lateral dentition of Sinornithosaurus were related to venom delivery (Gong et al., 2010, 2011). The dentition of the possibly venomous Varanus priscus is, to our knowledge, the closest analogue to the dromaeosaurid condition in terms of tooth type (i.e., ziphodont), denticle morphology and crown ornamentation (i.e., longitudinal ridges/grooves). Based solely on the fact that Varanus and some dromaeosaurids show some morphological convergence (and not on other anatomical features proposed by Gong et al. (2010, 2011) such as a 'subfenestral fossa' housing an ascinar venom gland), it is plausible that some theropods may have been venomous animals. For a different opinion, see Gianechini et al. (2011a).

The grooved teeth present in Unenlagiinae may indicate fish-eating behavior, since these dromaeosaurids lived in proximity to fluvial systems (Gianechini et al., 2011b). However, the teeth of piscivorous tetrapods such as crocodiles, marine reptiles, pterosaurs and spinosaurids are fluted and do not possess wide longitudinal grooves bounded by one or two poorly delimited ridges.
Based on the tooth morphology, a piscivorous lifestyle of dromaeosaurid theropods, although evidenced in Microraptor (Xing et al., 2013b), is therefore poorly supported in Unenlagiinae.

\section{Longitudinal grooves}

The presence of longitudinal grooves on the crown, which differ from lingual/labial depressions, flutes and concave surfaces, is poorly documented in theropods and may suggest the scarcity of this feature among these dinosaurs. A longitudinal groove on the mesiolingual surface of the crown has been noted in the lateral tooth of an abelisaurid (Hendrickx and Mateus, 2014a). Several authors have noted a groove in the vicinity of the mesial carina on the lingual surface of the mesial teeth. This is, for instance, the case in the paravians Linhevenator (Xu et al., 2011a), Sinornithosaurus (Xu and $\mathrm{Wu}, 2001$ ) and Urbacodon (Averianov and Sues, 2007). Yet, they better correspond to a mesiodistally narrow and well-delimited concave surface and are here reported in the section on $\mathrm{J}$ shaped cross-section. A similar groove can be observed in some mesial teeth of the metriacanthosaurid Sinraptor (IVPP V10600) and the allosaurid Allosaurus (UMNH VP 1251). Longitudinal grooves have also been observed on the crowns of Byronosaurus (Makovicky et al., 2003), as well as Austroraptor and Buitreraptor by Gianechini et al. (2011b), yet they result from the longitudinal ridges delimiting them, and we only consider the presence of ridges in Austroraptor, Buitreraptor, and Byronosaurus. An indeterminate tyrannosauroid from the Cenomanian of Utah also presents the particularity of having a longitudinal groove centrally positioned on the lingual side of a single mesial isolated crown (Zanno et al., 2019). Like these authors, who regard this dental feature as autapomorphic, we did not observe such groove in the mesial dentition of any other theropods. Finally, faint longitudinal grooves are present on the apex of a crown of Mononykus.

\section{Basal striations}

Basal striations, forming short parallel grooves extending apicobasally on the crown base, have been reported in herrerasaurids (Hendrickx and Mateus, 2014a) and the basal tyrannosauroid Proceratosaurus (Rauhut et al., 2010; Appendix 6.27). In the herrerasaurids Herrerasaurus (PVSJ 407; mx10) and Ischisaurus (MACN 18.060; pm1), which may represent the same taxon (Novas, 1992), these striations are numerous, closely packed, and restricted to some teeth. Although 


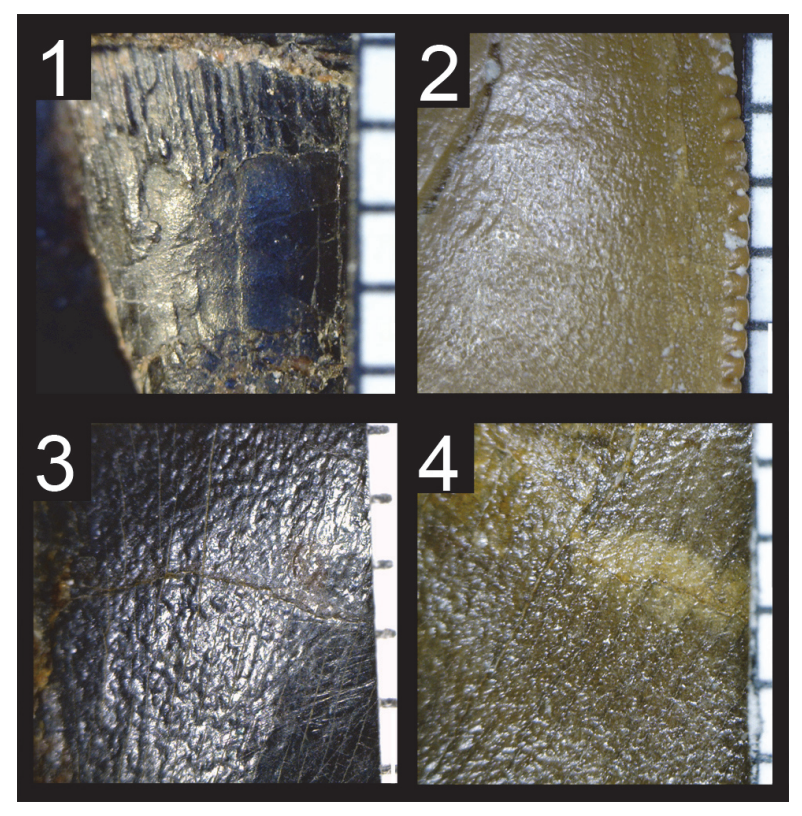

FIGURE 23. Irregular enamel texture of non-avian Theropoda. 1, Tenth left maxillary tooth of the herrerasaurid Herrerasaurus ischigualastensis (PVSJ 407) in labial view; 2, Isolated tooth of the abelisaurid Aucasaurus garridoi (MCF-PVPH-236) in lateral view; 3, Second premaxillary tooth of the allosaurid Allosaurus 'jimmadseni' (NHFO 455) in labial view; 4, Tenth maxillary tooth of the tyrannosaurid Tyrannosaurus rex (FMNH PR.2081) in labial view.

they likely result from taphonomic or anthropogenic factors in Herrerasaurus (they appear as scratches extending randomly apicobasally on the crown base and could correspond to striations from a layer of glue), basal striations appear to be genuinely present in the first premaxillary tooth of Ischisaurus where they, however, seem to be restricted to the root. These closely packed basal striations resemble and might be homologous to the plicidentine. A thorough investigation on the histology of teeth presenting such basal striations is, however, required to support this hypothesis.

Unlike herrerasaurids, basal striations form wide longitudinal depressions on the labial and/or lingual surfaces of most mesial teeth in Proceratosaurus (Rauhut et al., 2010). These basal striations do most likely not result from taphonomic processes given that they are restricted to the mesial dentition (pm1 to 3, dt1 and 3) and are present on undeformed teeth with well-preserved denticles and enamel surface texture (C.H. personal obs.). Apicobasally oriented striations at the base of the crown have also been noted in Spinosauridae by Mateus et al. (2011) but these basal striations could not be observed in any spinosaurid examined first hand.

\section{Irregular and smooth enamel texture}

With the exception of Spinosauridae, the crown enamel texture is rarely detailed in the theropod literature and we, therefore, rely on our own observations to explore this feature's distribution among non-avian theropods. An irregular non-oriented or smooth texture of the enamel (Figure 23) is present in most non-tetanuran saurischians, including Eoraptor (PVSJ 512), Herrerasaurus (PVSJ 407; Figure 23.1), Ischisaurus (MACN 18.060), Saltriovenator (Dal Sasso et al., 2018), Sinosaurus (IVPP V34) and most abelisauroids [e.g., Limusaurus; Wang et al.'s (2017a) data matrix] like Abelisaurus, Aucasaurus (Figure 23.2), Chenanisaurus (Longrich et al., 2017), Kryptops, Majungasaurus and Noasaurus (C.H. personal obs.). An irregular or smooth texture has also been observed in most non-dromaeosaurid neocoelurosaurs such as Compsognathus (MNHN CNJ 79), Scipionyx (SBA-SA 163760), Nqwebasaurus (AM 6040), Pelecanimimus (LHC 7777), Falcarius (UMNH VP 14528, 14545), Alxasaurus (IVPP V88402), Beipiaosaurus (IVPP V11559), Erlikosaurus (MPC-D 100-111), Shuvuuia (MPC-D 100-977), Caudipteryx (IVPP V12430), Almas (MPC-D 1001323), an unnamed basal troodontid (MPC-D 1001128), Byronosaurus (MPC-D 100-983), Troodon (DMNH 22337; 22837), Epidexipteryx (IVPP V15471) and Archaeopteryx (11 th specimen). In dromaeosaurids, this type of enamel texture has also been seen in Buitreraptor (MPCA 245), Velociraptor (AMNH 6515), Tsaagan (MPC-D 100-1015) and in the lateral dentition of Dromaeosaurus (AMNH 5356). Among non-neocoelurosaur tetanurans, an irregular texture of the enamel was observed in Erectopus (MNHN 2001-4), Sinraptor dongi (IVPP 10600) and S. hepingensis (ZDM T0024) as well as Irritator (SMNS 58022). Based on our observation, the latter does not appear to display the deeply veined texture of other spinosaurids (we could not identify the granular texture observed by Sues et al. (2002) in any maxillary tooth of Irritator). An irregular texture is also present in the crowns of some Tyrannosauridae such as Tyrannosaurus (FMNH PR.2081; Figure 23.4) and Zhuchengtyrannus (ZCDM V0031). This pattern of enamel texture has also been identified in some Allosaurus teeth (NHFO 455; Figure 23.3), the other crowns displaying a more oriented texture. In fact, Allosaurus appears to show a transitional condition between metriacanthosaurids with 
a non-oriented irregular enamel texture and the Allosauria (i.e., Allosauridae + Neovenatoridae + Carcharodontosauridae) with an oriented texture of the enamel. The presence of a smooth or irregular enamel surface texture is considered to be synapomorphic for Abelisauroidea, Metriacanthosauridae, and Neocoelurosauria (Appendix 6.28).

\section{Braided enamel texture}

A braided texture of the enamel, defined by alternating and interweaving grooves and sinuous ridges apicobasally oriented on the crown and never converging (Hendrickx et al., 2015c; Figure 24 ), is present in non-neocoelurosaur Neotheropoda, and can be observed in basal tetanurans such as Monolophosaurus (IVPP V84019), non-spinosaurid Megalosauroidea (e.g., Afrovenator, Dubreuillosaurus, Duriavenator, EustreptospondyIus, Megalosaurus, Piatnitzkysaurus, Torvosaurus), Allosauria (e.g., Acrocanthosaurus, Allosaurus, Carcharodontosaurus, Eocarcharia, Giganotosaurus, Mapusaurus, Megaraptor, Neovenator, Figure 24.2), Tyrannosauroidea (e.g., Albertosaurus, Alioramus, Daspletosaurus, Dilong, Eotyrannus, Gorgosaurus, Guanlong, Lythronax, Proceratosaurus, Raptorex, Teratophoneus, Tyrannosaurus; Figure 24.3) the basal coelurosaurs Bicentenaria (MPCA 866), Juravenator (JME Sch 200), Ornitholestes (AMNH 619) and Zuolong (IVPP V15912), and the basal alvarezsauroid Aorun (IVPP V15709). Such texture is also present in the basal saurischian Eodromaeus (PVSJ 561), the basal theropod Dracovenator (BP/1/5243), the non-averostran neotheropods Coelophysis (CMNH 81765), Dracoraptor (Martill et al., 2016, figure 9A-B), and Megapnosaurus rhodesiensis (USNM unnumbered), and non-abelisauroid ceratosaurs (e.g., Berberosaurus, Ceratosaurus, Genyodectes; Figure 24.1). An oriented texture has also been identified in the basal neotheropod 'Dilophosaurus' sinensis (the 'longitudinal striations in the enamel' of Xing 2012), the therizinosaur Segnosaurus (MPC-D 100-80), the dromaeosaurids Atrociraptor (TMP 1995.166.01), Bambiraptor (AMNH 30556), Deinonychus (YPM 5232 612), and Saurornitholestes (TMP 1994.12.844), and some mesial teeth of Dromaeosaurus (AMNH 5356; Figure 24.4) and Masiakasaurus (FMNH PR.2182, 2471). It is unknown whether a braided enamel texture is the ancestral condition in theropods, but the presence of such oriented pattern of the enamel is here considered synapomorphic for the clade Microraptorinae + Eudromaeosauria (Appendix 6.28).

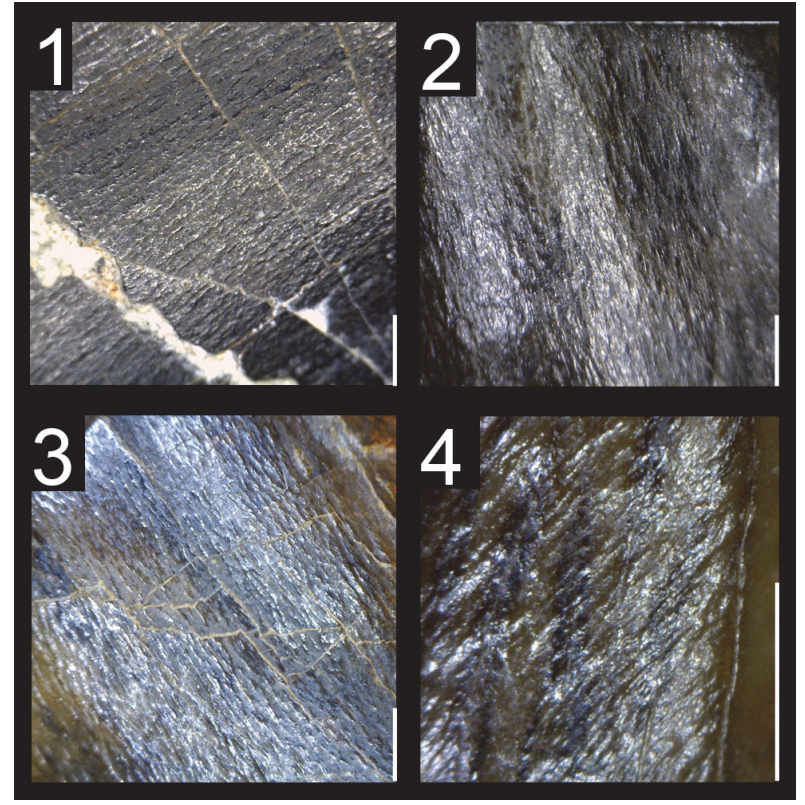

FIGURE 24. Braided enamel texture of non-avian Theropoda. 1, First right premaxillary tooth of the ceratosaurid Ceratosaurus nasicornis (UMNH VP 5278) in labial view; 2, Isolated tooth of the neovenatorid Neovenator salerii (MIWG 6348) in lateral view; 3, Third right maxillary tooth of the tyrannosaurid Lythronax argestes (UMNH VP 20 200) in labial view; 4, Isolated premaxillary tooth of the dromaeosaurid Dromaeosaurus albertensis (AMNH 5356) in lingual view. Scale bars equal 1 $\mathrm{mm}$.

\section{Veined and anastomosed enamel texture}

Veined enamel texture. A veined texture of the enamel is made of deep alternating grooves and long sinuous and/or dichotomized ridges obliquely or apicobasally oriented and converging basomesially or basodistally on the crown (Hendrickx et al., 2015d). It can be seen in the baryonychines Baryonyx walkeri (NHMUK PV R.9951; ML 1190; Figure 25.1) and Suchomimus tenerensis (e.g., MNN G35-9, G43-9, G73-73; Figure 25.2), and the spinosaurine Spinosaurus aegyptiacus (Hasegawa et al., 2010; MNHN MRS 478). Although noted by Sues et al. (2002) as exhibiting a granular texture similar to that of Baryonyx, the enamel texture of the spinosaurine Irritator challengeri (SMNS 58022 ) is smooth or displays an irregular pattern (Figure 21.3).

Anastomosed enamel texture. An anastomosed enamel surface texture, which consists of multiple ridges dividing and reconnecting in an irregular way, is present in Spinosaurus aegyptiacus (MSNM V4047, V6422; MNHN MRS 548; Figure 25.4) and in the premaxillary teeth of the basal ovi- 


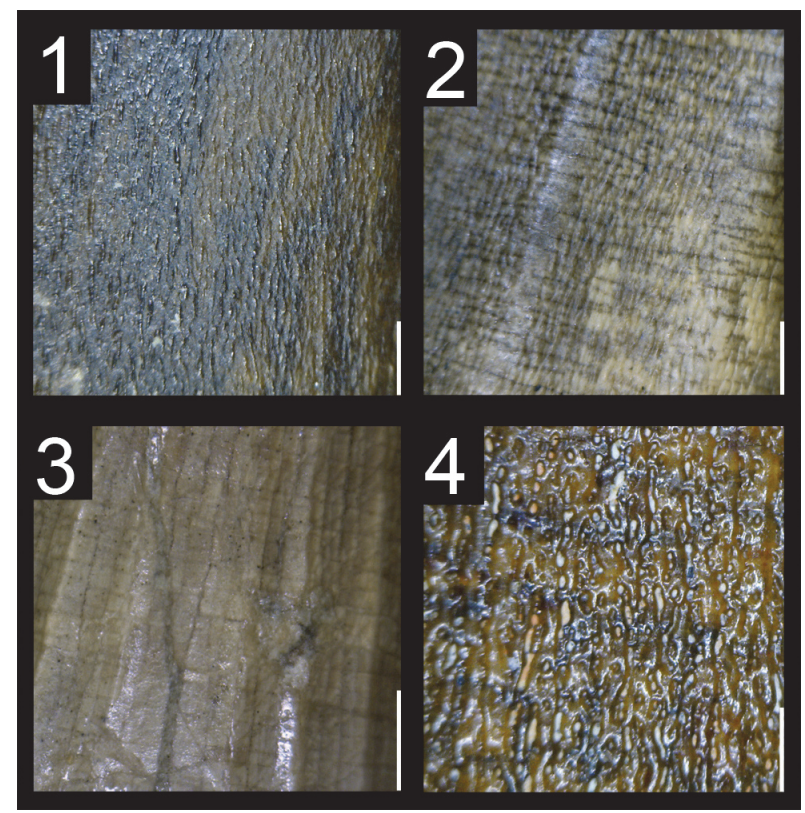

FIGURE 25. Enamel texture of spinosaurid teeth. 1, Veined enamel texture of an isolated tooth of the baryonychine Baryonyx walkeri (NHMUK PV R.9951 278) in lateral view; 2, Veined enamel texture of an isolated tooth of the baryonychine Suchomimus tenerensis (MNN G43-4) in lateral view; 3, Smooth enamel texture of a maxillary tooth of the spinosaurine Irritator challengeri (SMNS 58022) in lateral view; 4, Anastomosed enamel texture of an isolated tooth of the spinosaurine Spinosaurus aegyptiacus (MSNM V6422) in lateral view. Scale bars equal $1 \mathrm{~mm}$.

raptorosaur Incisivosaurus gauthieri (IVPP 13326). Such a pattern of the enamel texture has only been observed in these taxa and may correspond to an autapomorphy of Spinosaurus and Incisivosaurus. In spinosaurids, veined and anastomosed enamel textures are characterized by their strong basal curvature adjacent to the carinae (Hasegawa et al., 2010; Mateus et al., 2011), a feature that is not present in other theropods with a braided enamel texture of the crown (C.H. personal obs.).

Functional morphology and synapomorphy. We hypothesize that a veined and anastomosed texture of the enamel was developed in crowns subjected to particularly high mechanical stresses during feeding, a hypothesis which requires to be properly tested with tools like FEA. Spinosaurids are known to be at least partially piscivorous (e.g., Taquet, 1984; Charig and Milner, 1997; Ibrahim et al., 2014; Sales and Schultz, 2017), hunting on fish with hard scales like those of the actinopterygian Scheenstia, whose remains were found in the stomach region of Baryonyx (Charig and Milner, 1997). The coarse, complex and multiridged enamel may have conveyed particularly high resistance to the crowns, which were capable to resist high apicobasal stresses when piercing the scaly skins of fish. The same can be said for the putative herbivorous Incisivosaurus, whose premaxillary teeth show a strong similarity with the incisors of several mammalian lineages such as rodents ( $\mathrm{Xu}$ et al., 2002a). Some rodents are known to use their elongated incisors for gnawing (Ungar, 2010) and if the dentition of Incisivosaurus does not support such a feeding strategy (i.e., the anteriormost dentary portion is edentulous and the lateral dentition includes minute and labiolingually compressed folidont teeth unadapted for chewing), this basal oviraptorosaur likely used its premaxillary teeth to cut through particularly hard plant material. The presence of a deeply veined and/or anastomosed enamel surface texture is synapomorphic for Spinosauridae (Appendix 6.28).

\section{Procumbent, laterocumbent and retrocumbent teeth}

Procumbency in the premaxilla. Procumbent teeth (i.e., anterior inclination of the teeth; opposite of decumbent teeth, i.e., teeth with no inclination) are visible in the premaxilla of the basal saurischian Daemonosaurus (Sues et al., 2011), the basal coelurosaur Ornitholestes (Figure 26.1), the basal oviraptorosaur Similicaudipteryx ( $\mathrm{Li}$ et al., 2018, figure S3f), the scansoriopterygids Epidexipteryx (Zhang et al., 2008; Figure 26.2) and Yi (Xu et al., 2015b), in some specimens of the basal avialan Archaeopteryx (Rauhut et al., 2018), and to a lesser degree in the basal theropod Dracovenator (Yates, 2005). Based on the inclination of the alveoli, procumbent premaxillary teeth were also likely present in the noasaurid Masiakasaurus (Carrano et al., 2011) and the basal tetanuran Chilesaurus (SNGM-1935). Although the premaxillary teeth of the oviraptorosaur Caudipteryx were thought to be procumbent by Ji et al. (1998), the first right premaxillary tooth preserved in-situ in IVPP V12430 points ventrally, suggesting that the premaxillary teeth of Caudipteryx were decumbent.

Procumbency in the maxilla. Anteroventrally inclined teeth are present in the mesial maxillary teeth of many non-averostran neotheropods such as Coelophysis (Colbert, 1989), Dilophosaurus (Welles, 1984; Figure 26.4), 'Syntarsus' kayentakatae (Rowe, 1989), and Zupaysaurus (Ezcurra, 2007), the basal neotheropod 'Dilophosaurus' sinensis (ZLJT 0057), all Spinosauridae (Charig and Milner, 1997; Taquet and Russell, 1998; Sereno et al., 1998; Dal Sasso et al., 2005) that possess a 


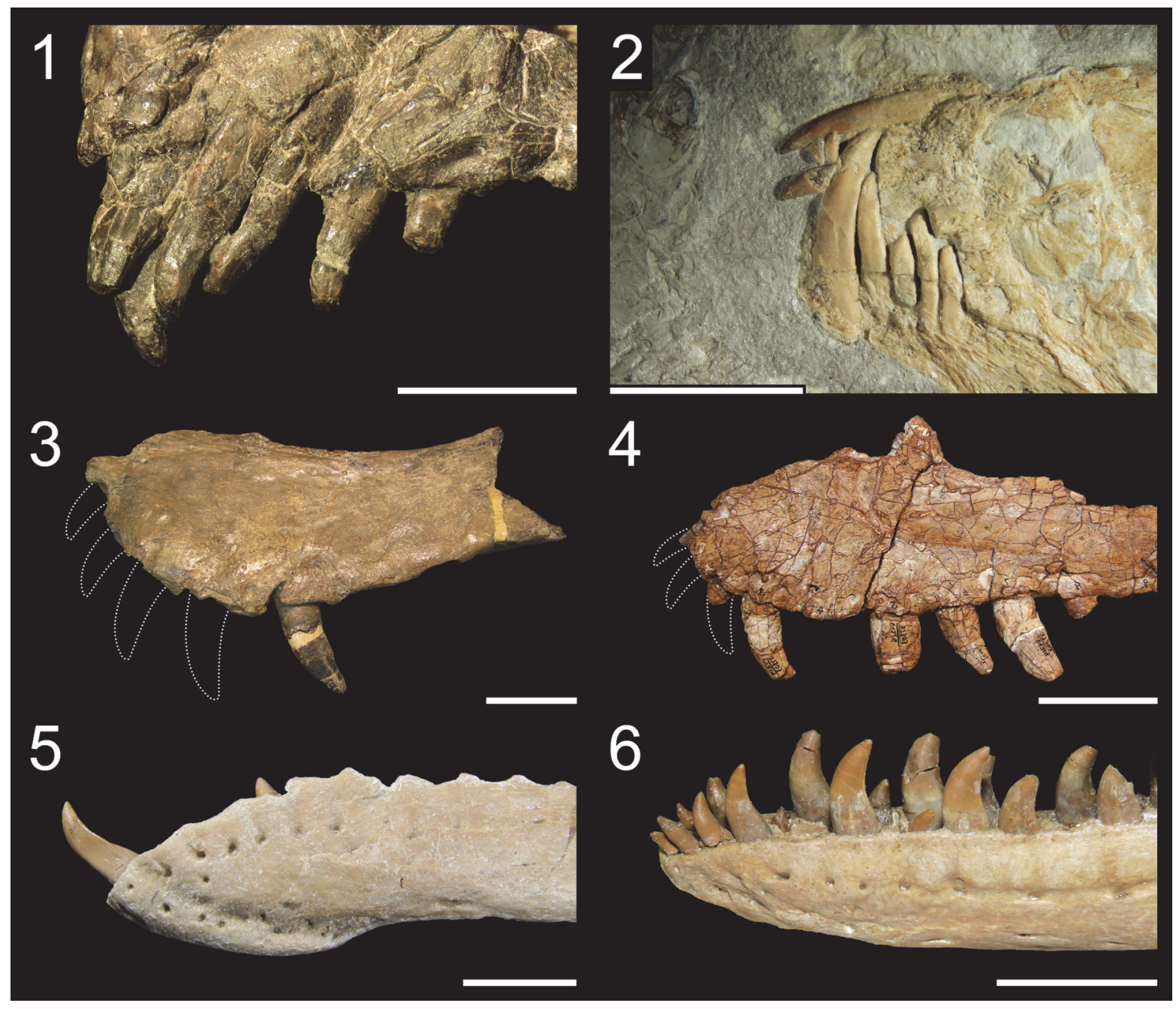

FIGURE 26. Procumbent teeth in non-avian Theropoda. 1, Procumbent premaxillary teeth of the right rostrum of the basal neocoelurosaur Ornitholestes hermanni (AMNH 619; reversed) in lateral view; 2, Procumbent premaxillary and dentary teeth of the right rostrum of the scansoriopterygid Epidexipteryx hui (IVPP V15471; reversed) in lateral view; 3, Hypothetical procumbent mesial maxillary teeth of the left maxilla of the spinosaurid Baryonyx walkeri (NHMUK PV R.9951) in lateral view; 4, Hypothetical procumbent mesial maxillary teeth of the right maxilla of the non-averostran neotheropod Dilophosaurus wetherilli (UCMP 37303; reversed) in lateral view; 5, Procumbent mesial dentary teeth of the left dentary of the noasaurid Masiakasaurus knopfleri (FMNH PR 2471) in lateral view; 6, Procumbent mesial dentary teeth of the right dentary of the proceratosaurid Proceratosaurus bradleyi (NHMUK PV R.4860; reversed) in lateral view. Scale bars equal $1 \mathrm{~cm}(1-2,5), 2 \mathrm{~cm} \mathrm{(6),} \mathrm{and} 5 \mathrm{~cm}(3,4)$.

sigmoid alveolar margin of the upper jaw (Figure 26.3), and in the basal coelurosaur Ornitholestes (AMNH 619). Procumbency has also been noted in the first maxillary tooth of the compsognathid Scipionyx (Dal Sasso and Maganuco, 2011) and the noasaurid Masiakasaurus (Carrano et al., 2002, 2011). An anteroventrally inclined lateral dentition has been observed in the maxilla of the basal tetanuran Chilesaurus (Novas et al., 2015, figure 2a), the basal ornithomimosaur Nqwebasaurus (Choiniere et al., 2012), the basal oviraptorosaur Similicaudipteryx ( $\mathrm{Li}$ et al., 2018, figure S3f), the scansoriopterygid Epidexipteryx (Zhang et al., 2008) and some specimens of Archaeopteryx (Rauhut et al., 2018). Chilesaurus and Nqwebasaurus' procumbency may, however, result from diagenetic factors (Choiniere et al., 2012). Procumbent mesial maxillary teeth is a synapomorphy for Spinosauridae (Appendix 6.30)

Procumbency in the dentary. Strongly procumbent teeth are seen in the anteriormost part of the dentary of the basal saurischian Daemonosaurus (Sues et al., 2011), the coelophysoids Coelophysis (NMMNH p42200; DMNS 39022; CM 81765), 


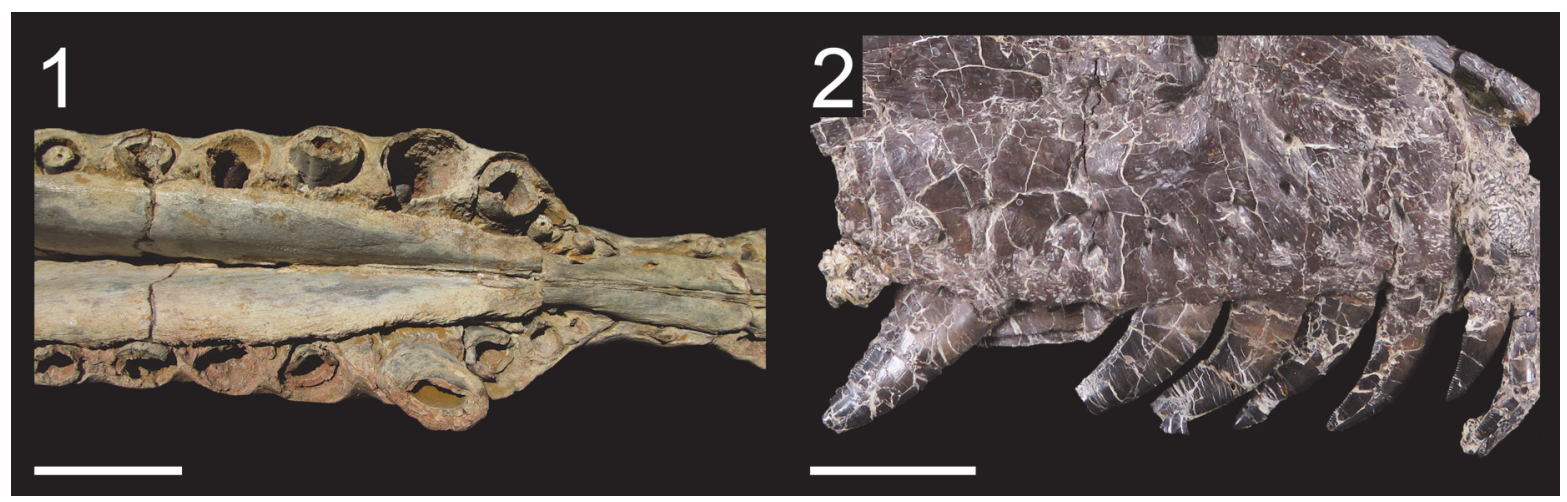

FIGURE 27. Laterocumbent and retrocumbent teeth in non-avian Theropoda. 1, Laterocumbent teeth (teeth facing ventrolabially) in the maxillae of the spinosaurid Spinosaurus aegyptiacus (MSNM V4047) in palatal view; 2, retrocumbent teeth (distally inclined teeth) in the cranium of the dromaeosaurid Deinonychus antirrhopus (YPM 5232) in labial view. Scale bars equal $10 \mathrm{~cm}(1)$ and $2 \mathrm{~cm} \mathrm{(2).}$

Megapnosaurus (Raath, 1977), the basal neotheropod Liliensternus (MB R.2175), the noasaurid Masiakasaurus (Carrano et al., 2002; Figure 26.5), the spinosaurid Spinosaurus (NHMUK VP R.16421), adult individuals of the dromaeosaurid Utahraptor (UMNH VP 20501; Kirkland et al., 2016, figure 9B) and the troodontid Daliansaurus (Shen et al., 2017a, figures 3 and 4A). Among Tyrannosauroidea, the proceratosaurid Proceratosaurus (Rauhut et al., 2010; Figure 26.6) and a single specimen of the basal pantyrannosaurian Dilong (IVPP V11979) also show this condition. Procumbent mesial dentary teeth are present, yet to a lesser degree (i.e., teeth less labiobasally inclined), in the basal saurischian Herrerasaurus (Sereno and Novas, 1994), the megalosaurid Duriavenator (Benson, 2008) and the tyrannosaurids Albertosaurus, Daspletosaurus, Tarbosaurus and Tyrannosaurus (Bakker et al., 1988; Carr and Williamson, 2004). Slightly procumbent mesialmost dentary teeth have also been observed in the basal maniraptoriform Ornitholestes (AMNH 619), the alvarezsauroid Haplocheirus (Choiniere et al., 2010b), the basal oviraptorosaur Incisivosaurus (Balanoff et al., 2009), and the dromaeosaurids Sinornithosaurus (Xu and $\mathrm{Wu}, 2001$ ), possibly Saurornitholestes (TMP 88.121.39), and some Microraptor specimens (BMNHC PH881; Xing et al., 2013b). In the basal tetanuran Chilesaurus (Novas et al., 2015), the ornithomimosaur Shenzhousaurus (Ji et al., 2003), the basal oviraptorosaur Similicaudipteryx (Li et al., 2018, figure S3f), and the scansoriopterygids Epidexipteryx (Zhang et al., 2008; Figure 26.2) and Yi (Xu et al., 2015b), the whole dentition of the dentary is slightly to strongly procumbent. If the mandibular procumbency in Similicaudipteryx and Epidexipteryx clearly results from the downturned symphyseal region of the dentary, that seen in Chilesaurus might possibly be due to diagenetic factors.

Laterocumbency. Theropod teeth can also be strongly inclined laterally/labially within the toothbearing bones (C.H. personal obs.), a condition here referred to as laterocumbent (Figure 27). Laterocumbent premaxillary and maxillary teeth seem to be synapomorphic for the Spinosaurinae as this condition is seen in Angaturama (USP GP/2T-5), Irritator (SMNS 58022) and Spinosaurus (MSNM V4047; Figure 27.1). The teeth of other spinosaurid taxa such as the baryonychine Baryonyx are decumbent or procumbent. A laterocumbent dentition is also present in some rhamphorhynchid (e.g., Rhamphorhynchus; Bonde and Leal, 2015), ornithocheiran (e.g., Ornithocheirus; Rodrigues and Kellner, 2013, figures 4-6, 11-14) and ctenochasmatid pterosaurs (e.g., Ctenochasma; Jouve, 2004) as well as in many plesiosaurs such as cryptoclidids (e.g., Cryptoclidus; Brown and Cruickshank, 1994, figure 5) and plesiosaurids (e.g., Morturneria, Aristonectes; O'Keefe et al., 2017).

Retrocumbency. Some theropods have posteriorly/distally inclined crowns along the jaws, a condition here referred to as retrocumbent. The apex of fully erupted retrocumbent teeth is strongly distally offset from the distal margin of the crownbase. Retrocumbent premaxillary teeth are present in juvenile individuals of Limusaurus (Wang et al., 2017a, figure 2A and B) as well as Eoraptor (PVSJ 512), Juravenator (Chiappe and Göhlich, 2010, figure 10A) and several basalmost neotheropods such as Dilophosaurus (Welles, 1984, figure 4), Dracovenator (BP/1/5278), 'Syntarsus' kayentakatae (Tykoski, 1998, figure 6) and some Coelophysis specimens (e.g., NMMNH p42200) due to the 


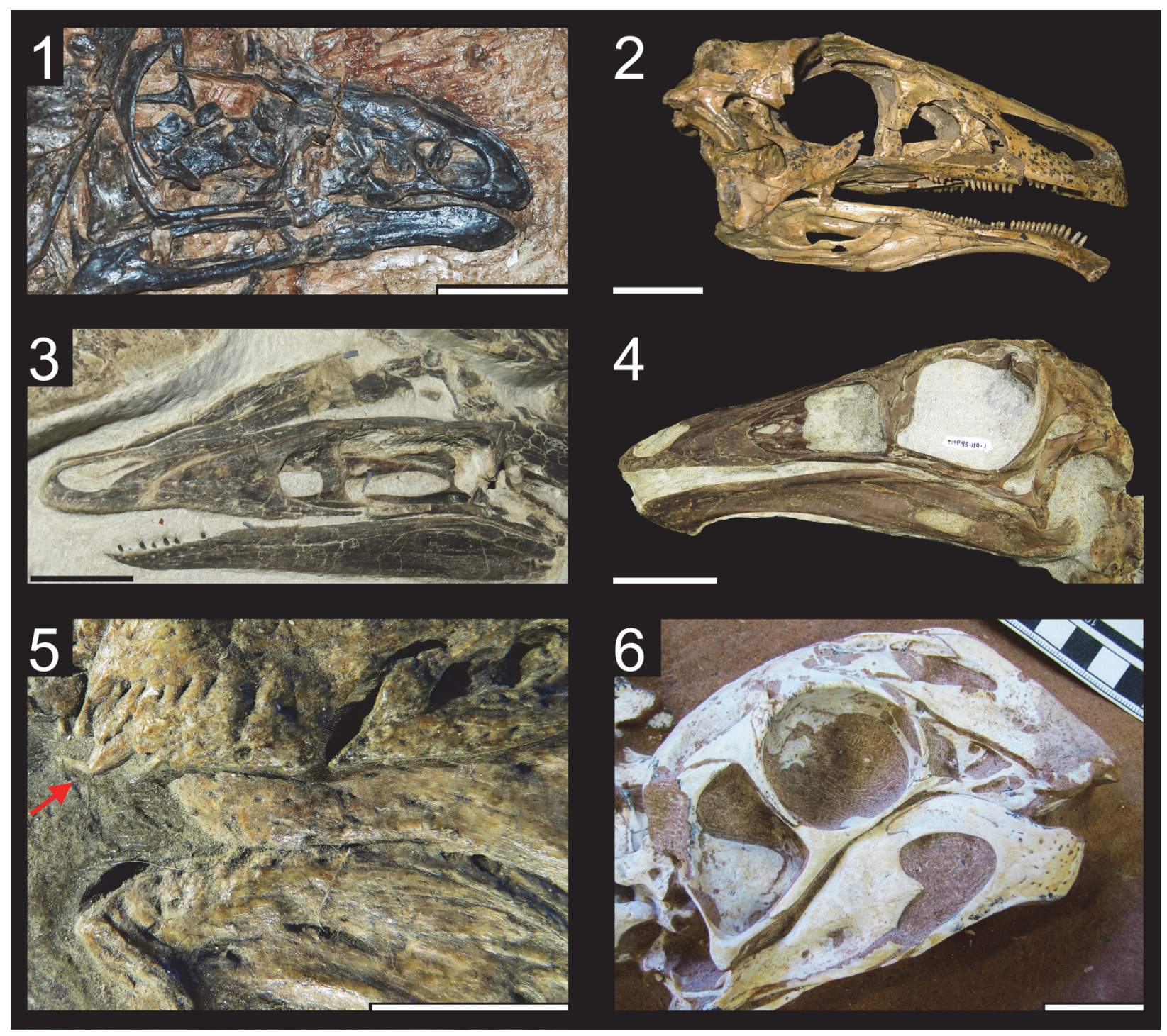

FIGURE 28. Partial and complete edentulism in non-avian Theropoda. 1, Skull of the toothless noasaurid Limusaurus inextricabilis (IVPP V15523) in right lateral view; 2, skull of the therizinosaurid Erlikosaurus andrewsi (MPC-D 100111 ) with a premaxilla and anterior portions of the maxilla and dentary toothless in right lateral (cranium) and medial (mandible) views; 3, skull of the basal ornithomimosaur Shenzhousaurus orientalis (NGMC 97-4-002), with a dentulous anterior portion of the dentary, in left laterodorsal view; 4, Skull of the toothless ornithomimosaurid Ornithomimus edmontonicus (TMP 1995.110.01) in left lateral view; 5, premaxilla, maxilla and dentary of the caudipterid Caudipteryx zoui (IVPP V12430), with a toothed anterior portion of the premaxilla, in left lateral view; $\mathbf{6}$, Skull of the toothless oviraptorid Khaan mckennai (MPC-D 100-1002) in right lateral view. Scale bars equal $1 \mathrm{~cm}(5), 2 \mathrm{~cm}(3), 3 \mathrm{~cm}(1,6)$, and $5 \mathrm{~cm}(2,4)$.

slight posteroventral inclination of the premaxilla. Retrocumbent maxillary teeth have been observed in the coelophysoid Panguraptor (You et al., 2014, figure 2), the megalosaurid Sciurumimus (Rauhut et al., 2012), the dromaeosaurids Atrociraptor, Bambiraptor, and Deinonychus (Figure 27.2), and an undescribed dromaeosaurine (UC uncatalogued) from the Ulansuhai Formation of Inner Mongolia (Watanabe and Sereno, 2010). The ret- rocumbent premaxillary and maxillary teeth seen in one specimen of Sinovenator (IVPP V12615; Xu et al. 2002b, figure 1a) are here interpreted as resulting from diagenetic factors given that the cranial dentition is clearly decumbent in the undescribed specimen of Sinovenator IVPP V12632.

Functional morphology. Procumbent mesial teeth are common in piscivorous animals including crocodiles, fish, and pterosaurs (Xing et al., 
2013b). A forward-projecting mesial tooth arrangement seems to be adapted for the prehension of small prey such as invertebrates and small vertebrates such as fish (Carrano et al., 2002). In coelophysoids and spinosaurids, procumbent maxillary teeth result from the sigmoid ventral margin of the maxilla and are suitable for gripping small to moderate-sized prey transversally through slashing bites (Charig and Milner, 1997; Therrien et al., 2005). The sigmoid margin of the upper jaw, present in many crocodylomorphs, would also have enhanced the ability for holding prey and/or tearing their flesh (Russell and Wu, 1997). Bakker and colleagues (1988) suggested that the mesialmost procumbent dentary teeth of tyrannosaurids allowed to bite a chunk of bone and flesh off a prey's body part that was gently curved such as the rib-cage and abdominal wall when the head was moving forward. In ornithomimosaurs with mesially inclined teeth like Nqwebasaurus and Shenzhousaurus, in which a herbivorous diet has been inferred (Makovicky et al., 2004; Zanno and Makovicky, 2011; Choiniere et al., 2012), procumbency is most likely adapted for the prehension of vegetation, perhaps for branch raking/stripping as suggested for other saurischians like Diplodocus (e.g., Barrett and Upchurch, 1994; Upchurch and Barrett, 2000). The procumbency seen in the lateral dentition of ornithomimosaurs and other maniraptoriforms may also result from a trophic shift from carnivory to herbivory (Zanno and Makovicky, 2011; Choiniere et al., 2012). Laterocumbent and retrocumbent teeth conversely enable to firmly hold and keep small struggling and/or slippery prey such as lizards and fish, or to keep a firm and immobile bite when teeth are deeply inserted into the flesh of a larger prey item.

\section{Partial and complete edentulism}

Premaxilla. Among toothed theropods, partially edentulous premaxillae combined with toothed maxillae and dentaries is an autapomorphic condition of juvenile individuals of the noasaurid Limusaurus (Wang et al., 2017a, figure 2A and B), in which a single premaxillary tooth erupts in the posterior part of the premaxilla. Fully edentulous premaxillae with dentulous maxillae and dentaries are shared by Therizinosauroidea such as Erlikosaurus (Clark et al., 1994; Lautenschlager et al., 2014; Figure 28.2) and Jianchangosaurus ( $\mathrm{Pu}$ et al., 2013). Toothed premaxillae with edentulous maxillae and dentaries characterize the basal oviraptorosaur Caudipteryx (Ji et al., 1998; Zhou et al., 2000; Figure 28.5) whose teeth are restricted to the anterior portion of the premaxilla. Although premaxillary teeth were revealed to be present in an Avimimus specimen (Watabe et al., 2000), the premaxilla is edentulous in all specimens of this taxon (Funston et al., 2016; Tsuihiji et al., 2017; Funston personal comm. 2019). An edentulous premaxilla is synapomorphic for Ornithomimosauria more derived than Pelecanimimus, Therizinosauroidea and Caenagnathoidea (Appendix 6.32).

Maxilla. An edentulous anterior portion of the maxilla is present in the therizinosaurid Erlikosaurus (Lautenschlager et al., 2014) and in ontogenetic stage II of Limusaurus (Wang et al., 2017a, figure 2B). An edentulous posterior portion of the maxilla (i.e., tooth row extending only on the anterior $75 \%$, or less, of the maxilla) can be seen in ontogenetic stage II of Limusaurus (Wang et al., 2017a, figure 2B), and the basal ornithomimosaurs Nqwebasaurus (Choiniere et al., 2012) and Pelecanimimus (Pérez-Moreno et al., 1994). Such condition is also seen in the alvarezsaurid Shuvuuia (Chiappe et al., 1998), the therizinosaur Erlikosaurus (MPC-D 100111), the oviraptorosaur Similicaudipteryx ( $\mathrm{Li}$ et al., 2018, figure S3f), many jinfengopterygine and troodontine troodontids such as MPC-D 100-1128, IVPP V20378, Almas, Byronosaurus, Gobivenator, Saurornithoides and Zanabazar (Pei et al., 2017b), and the scansoriopterygids Epidexipteryx (IVPP V15471) and Yi (Xu et al., 2015b). A toothless posterior portion of the maxilla is a synapomorphy of Ornithomimosauria, whereas an edentulous maxilla is synapomorphic for Ornithomimosauria more derived than Pelecanimimus, and the oviraptorosaur clade gathering Caudipteryx and Caenagnathoidea (Appendix 6.33).

Dentary. Edentulous premaxillae and maxillae, combined with toothed dentaries, are present in the basal ornithomimosaurs Harpymimus (Kobayashi and Barsbold, 2005), Hexing (Jin et al., 2012) and Shenzhousaurus (Ji et al., 2003; Figure 28.3). The absence of teeth in the anteriormost part of a dentulous dentary has been recorded in Eoraptor (Sereno et al., 2013), therizinosauroids (Beipiaosaurus, Erlikosaurus, Neimongosaurus, Segnosaurus; Zanno et al., 2016; Liao and Xu, 2019; Figure 28.2), basal oviraptorosaurs (Incisivosaurus, Protarchaeopteryx, Similicaudipteryx; Zanno and Makovicky, 2011) and juvenile individuals of Limusaurus (Wang et al., 2017a, figure 2A and $B$ ). An edentulous anteriormost portion of the dentary was also noted in Alvarezsauridae by Longrich et al. (2009). Although possible, the source used by these authors (i.e., Suzuki et al., 2002) does not seem to provide this information, and the 
missing anterior extremity of the dentary of Shuvuuia (Dufeau, 2003), the only alvarezsaurid preserving in situ teeth in the anterior portion of the mandible, does not allow to confirm this statement either. The posterior portion of the dentary is devoid of teeth (i.e., more than $25 \%$ of the posterior portion of the dentary is toothless) in ontogenetic stage II of Limusaurus (Wang et al., 2017a, figure 2B), the basal ornithomimosaurs Harpymimus (Kobayashi and Barsbold, 2005), Hexing (Jin et al., 2012) and Shenzhousaurus (Ji et al., 2003; Figure 28.3), the oviraptorosaur Similicaudipteryx (Li et al., 2018, figure S3f), and possibly all scansoriopterygids (e.g., IVPP V15471; STM 31-2; Zhang et al., 2002, figure 1b). An edentulous posterior portion of the dentary is synapomorphic for Ornithomimosauria more derived than Pelecanimimus, whereas a toothless anteriormost portion of the dentary is a synapomorphy of Oviraptorosauria and Therizinosauroidea (Appendix 6.33). The oviraptorosaur clade gathering Caudipteryx and Caenagnathoidea share the apomorphic feature of having an edentulous dentary (Appendix 6.34).

Complete edentulism. Toothless non-avian theropods are restricted to three clades, namely: Ceratosauria with the noasaurid Limusaurus (Xu et al., 2009; Figure 28.1), Ornithomimosauria with all ornithomimoids (Kobayashi and Barsbold, 2005; Makovicky et al., 2010; Lee et al., 2014b; Figure 28.4), and Oviraptorosauria with all caenagnathoids (Avimimus + Caenagnathidae + Oviraptoridae, sensu Qiu et al., 2019; Figure 28.6). In Limusaurus, however, teeth are borne by hatchling and juvenile individuals and disappear throughout ontogeny (Wang et al., 2017a). Palatal teeth have been retained by the basal sauropodomorphs Eoraptor (Sereno et al., 2013) and Buriolestes (Müller et al., 2018) as well as the putative nonneotheropod theropod Eodromaeus (Martínez et al., 2011; Baron et al., 2017; Müller et al., 2018, fourth phylogenetic analysis). Theropods more derived than Eodromaeus, therefore, have an edentulous pterygoid. If Eodromaeus is, however, a non-theropod saurischian, as recently recovered by Langer et al. (2017) and Müller et al. (2018, first and fifth phylogenetic analyses), a toothless pterygoid is synapomorphic to theropods. As for the rest of the jaw, a fully edentulous skull is synapomorphic for Ornithomimoidea and Caenagnathoidea.

Functional morphology. Tooth loss and edentulism in theropods have been recently investigated by Zhou and Li (2009), Davit-Béal et al. (2009), Louchart and Viriot (2011), Zanno and Makovicky (2011), Lautenschlager et al. (2013), Meredith et al. (2014), Bhullar et al. (2016), Wang et al. (2017b), Erickson et al. (2017) and Yang and Sander (2018). The classical reasons for the origin of edentulism are related to developmental economy and for food acquisition. Davit-Béal et al. (2009) pointed out that in tetrapods the loss of teeth (or enamel) was preceded by the progressive replacement of an anatomical identity that allowed food uptake and oral processing (e.g., beak, baleen, ever-growing teeth, known as hypselodonty. Such anatomical entities are called by the authors as secondary tools). Positive selection of these secondary tools led to relaxed functional constraints on the teeth evolution. However, various other hypotheses have been proposed. Namely, Zhou and Li (2009) while describing the basal bird toothless Zhongjianornis yangi related its edentulism to the selective pressure for weight reduction. However, this hypothesis seems to lack much support as not only teeth represent a minor proportion of head weight, but also many other edentulous clades are characterized by large body masses (e.g., ornithomimosaurs, turtles and whales).

According to Lautenschlager et al. (2013) who focused on Therizinosauria, large-scale dietary changes were a trigger to specializations we see in the masticatory apparatus of maniraptoriform theropods including tooth loss as in birds, their descendants. Through FEA of biomechanical models of Erlikosaurus, the authors have concluded that a keratinous rhamphotheca was capable of dissipating stresses and strains generated by muscle action during jaw adduction. Along these lines, Zanno and Makovicky (2011) causally related partial to complete edentulism to herbivory. However, they argue that efficient oral processing is a negative selective factor for edentulism (Wings and Sander, 2007; Zanno and Makovicky, 2011), as various other herbivorous dinosaur clades actually developed dental batteries. In order to understand if edentulism aroused convergently in two or more modern bird lineages, Meredith et al. (2014) analyzed for the presence of inactivating mutations on six dentine- and enamel-forming genes in multiple bird clades and a crocodilian outgroup. All enameland dentine-forming genes were, in fact, deactivated in all bird lineages but present in the crocodilian outgroup, pointing towards a single deactivation of these genes in the common ancestor to all birds.

In another study, Wang et al. (2017a) studied nearly 20 specimens of the unorthodox ceratosaurian species Limusaurus inextricabilis concerning 
its developmental morphological patterns. The juvenile Limusaurus possesses fully toothed jaws, but the teeth are completely lost in adults. This rare example of radical ontogenetic edentulism happens through an unusual mechanism, compared to Cretaceous ornithurine birds and other theropods. The teeth are lost on the anterior and posterior portions of the jaws coupled with tooth loss by the absence of replacement teeth eruption. The authors hypothesize that the early cessation of tooth replacement in Limusaurus may have resulted from the regression of successional lamina. Wang et al. (2017a) also point out to heterochronic mechanisms for the retardation and eventual truncation of tooth development, as exemplified by Limusaurus. In a follow-up paper, Wang et al. (2017b) provide evidence for the hypothesis of vestigialization of alveoli as part of a process towards edentulism. Caenagnathid oviraptorosaurs and the Cretaceous bird genus Sapeornis show a system of vestiges that connects via foramina with a dorsally closed canal homologous to alveoli, which exemplifies an evolutionary stage more advanced than that in Limusaurus. The authors further substantiate the hypothesis that heterochrony plays a crucial role in the progressively earlier postnatal and embryonic truncation of odontogenesis. Eventually, the absence of teeth leads to the development of a secondary tool: a keratinous beak.

More recently, Erickson et al. (2017) developed a new approach to determine incubation periods in fossil non-avian dinosaurs by counting the number of growth lines in fossil embryonic teeth of two ornithischian dinosaurs: Hypacrosaurus and Protoceratops. The estimated incubation periods of these ornithischians were close to those of reptiles (i.e., long incubation periods), contrasted with those of birds, which last for only 11-85d. It is noteworthy that this approach could be tested on other dinosaurs closer to the bird lineage (e.g., Torvosaurus; Araújo et al., 2013). Yang and Sander (2018) suggested that the incubation period is a selection factor for edentulism or, in other words, the selection for tooth loss was a side effect of the selection for fast embryo growth and thus shorter incubation. This hypothesis, however, lacks the support of experimental evidence.

\section{CONCLUSIONS}

By investigating the distribution of dental features in non-avian theropods, this study reveals that isolated theropod teeth can be better diagnosed at 'family' and 'sub-family' level clades than genus-level taxa based on qualitative data. The dentition of theropod dinosaurs is highly homoplastic: there is a morphological convergence among distantly related clades that have adopted similar dietary preferences. Nonetheless, dental characters often provide useful grouping information, and the isolated crowns of a few taxa such as Majungasaurus, Piatnitzkysaurus and Acrocanthosaurus provide sufficiently distinct morphologies to be used as high-precision biostratigraphic markers.

Crown ornamentations and enamel surface texture and microstructure are the least homoplastic dental features possibly because they are less prone to evolve than other dental features under similar evolutionary pressures. A mesial dentition bearing a longitudinal ridge, a mesial carina spiraling or reaching the cervix, an unserrated distal carina and the cross-sectional outline in mesial teeth as well as fluted lateral teeth, lateral crown height and a basal constriction at the cervix in the lateral dentition are the dental characters that provide the most important grouping information. On the other hand, procumbent teeth, the mesial and distal denticle morphology, a labially deflected distal carina, a significant difference in size between mesial and distal denticles, interdenticular sulci and transverse and marginal undulations provide particularly little grouping information. Yet, a combination of these dental features can certainly refine the identification of isolated teeth up to the genus level for some taxa.

Major evolutionary transitions in the dentition of theropod dinosaurs occurred with the emergence of Spinosauridae, Allosauroidea, Tyrannosauroidea and Maniraptoriformes. These dental transitions are functionally linked to an anteroposterior shortening of the premaxillae in allosauroids and tyrannosauroids, and to a switch in dietary preferences towards piscivory and herbivory in spinosaurids and maniraptoriforms, respectively. Although dental characters have high levels of homoplasy, for some large datasets they are no more or less reliable, on average, than other character systems. This argues for their continued inclusion into phylogenetic datasets for theropod dinosaurs. Moreover, the functional implications of tooth features allow for the more nuanced study of their homoplasy in a comparative biology framework.

\section{ACKNOWLEDGEMENTS}

Several institutions of Europe, United States, China, Argentina and Qatar were visited in order to conduct this research, and we are deeply appreciative to S. Mateus (ML), L. Jacobs (SMU), D Winkler 
(SMU), A. Fiorillo (DMNH), R. Tykoski (DMNH), P. Sereno (Uni. Chicago), Peter Makovicky (FMNH), W. Simpson $(\mathrm{FMNH}), \mathrm{M}$. Lamanna $(\mathrm{CMNH}), \mathrm{A}$. Henrici (CMNH), M. Carrano (USNM), M. BrettSurman (USNM), A. Millhouse (USNM), S. Chapman (NHMUK), P. Barrett (NHMUK), P. Jeffery (OUMNH), S. Hutt (MIW), R. Allain (MNHN), R. Schoch (SMNS), H. Siber (SMA), C. Dal Sasso (MSNM), A. Kramarz (MACN), F. Novas (MACN), M. Reguero (MLP), R. Barbieri (MPCA), L. Salgado (MUCPv-CH), J. Ignacio Canale (MUCPv-CH), R. Coria (MCF-PVPH), C. Succar (MCF-PVPH), J. Calvo (CePaLB), J. Porfiri (UNDC), R. Martínez (PVSJ), C. Mehling (AMNH), M. Norell (AMNH), D. Krauze (SBU), J. Groenke (SBU), P. Brinkman (NCSM), L. Zanno (NCSM), J. Sequeira (LNEG), Fareed Krupp (QMA), Khalid Hassan Al-Jaber (QMA), Sanker S.B (QMA), Yves Dutour (MHNA), T. Tortosa (MHNA), J. Powell (PVL), P. Ortiz (PVL), R. Gonzalez (PVL), B. Zipfel (ESI) and S. Jirah (ESI), X. Xing (IVPP), Z. Fang (IVPP), C. Sullivan (IVPP), L.Feng (IVPP), L. Zhang (IVPP), J. O'Connor (IVPP), A. Folie (IRSNB), P. Godefroit (IRSNB), O. Rauhut (BSPG), G. Rößner (BSPG), F. Holwerda (BSPG), M. Kölbl-Ebert (JME), B. Britt (BYU), C. Levitt-Bussian (UMNH) R.B. Irmis (UMNH), J. Sertich (DMNS), L. Ivy (DMNS), B. Strilisky (TMP), B. Sanchez (TMP), D. Brinkman (TMP), D. Henderson (TMP) and D.L. Brinkman (YPM) for access to the specimens and the help provided during the visit of their collections. We are particularly thankful to X. Xing, P. Sereno, F. Novas, M. Norell, F. Krupp, K. Hassan Al-Jaber, and S.B. Sanker who gave us access to unpublished material.

Many photos of theropod teeth were also kindly shared by R. Delcourt (Uni. São Paulo), S. Evers (Uni. Oxford), M. Ezcurra (LMU), M. Loewen (UMNH), J. Stiegler (George Washington Uni.), L. Zanno (NCSM), M. Lamanna (CMNH), O. Rauhut (Ludwig-Maximilians-Uni.), S. Brusatte (Uni. Edinburgh), C. Foth (Ludwig-Maximilians-Uni.), A. Cau (MGG), X. Wu (Canadian Museum of Nature), P. Currie (Uni. of Alberta), S. Nesbitt (Virginia Tech),
P. Makovicky (FMNH), D. Pol (MPEF), J. Canale (MUCPv-CH), C. Dal Sasso (MCSN), L. Feng (IVPP), R. Molnar (MNA), R. Benson (Uni. Oxford), M. Pittman (Uni. Hong Kong), V. Radermacher (Wits Uni.), C. Sullivan (IVPP), M. Ellison (AMNH), E. Malafaia (MNHNC), E. Tschopp (AMNH), D. Eddy (Uni. Texas), V. Shneider (NCSM), K. Peyer (MNHN), D. Larson (Uni. Toronto), M. Mortimer, S. Maganuco (MSNM) and A. Yates (MCA) and the authors would like to address their sincere thanks to these people and their generosity.

We acknowledge the use of the Willi Hennig Society edition of TNT for the cladistic analysis and Phylopic for the theropod silhouettes, and thank S. Hartman, G.S. Paul, M. Martyniuk, J. Headden, T.M. Keesey, J. Garza, V.-V. Sinkkonen, N. Tamura, Funkmonk, C. Dyke, T. Tischler, Conty, and Traver for accepting to use or sharing their artworks on Phylopic. The authors wish to thank R. Molnar (MNA), three anonymous reviewers and editors L. Wilson and J. Louys for extensive comments that greatly improved this manuscript. G. Apud Martin and her parents J. and G. Apud Martin are also warmly thanked for the corrections of the text and/or the tremendous support. This research was financially supported by the Fundação para a Ciência e a Tecnologia (FCT) doctoral scholarship SFRH/BD/62979/2009 (Ministério da Ciência, Tecnologia e Ensino superior, Portugal), and the Postdoctoral Fellowship from the University Research Committee (URC) of the University of the Witwatersrand to C.H. The support of the DST-NRF Center of Excellence in Palaeosciences (CoE-Pal) towards this research (grant PPD2015/17CH and CoE2017-038 as) is hereby acknowledged. Opinions expressed and conclusions arrived at, are those of the authors and are not necessarily to be attributed to the CoE. J.N.C. was supported by the Palaeontological Scientific Trust and its Scatterlings of Africa Programmes, and by the Friedel Sellschop Award administered by the University of the Witwatersrand. C.H. dedicates this work to E. Buffetaut.

\section{REFERENCES}

Abler, W.L. 1992. The serrated teeth of tyrannosaurid dinosaurs, and biting structures in other animals. Paleobiology, 18:161-183.

Abler, W.L. 1997. Tooth serrations in carnivorous dinosaurs, p. 740-741. In Currie, P.J. and Padian, K. (eds.), Encyclopedia of Dinosaurs. Academic Press, San Diego, California. Abler, W.L. 1999. The teeth of the tyrannosaurs. Scientific American, 281:50-51. 
Agnolin, F.L., Motta, M.J., Brissón Egli, F., Lo Coco, G., and Novas, F.E. 2019. Paravian phylogeny and the dinosaur-bird transition: an overview. Frontiers in Earth Science, 6:1-252. https://doi.org/10.3389/feart.2018.00252

Allain, R. and Taquet, P. 2000. A new genus of Dromaeosauridae (Dinosauria, Theropoda) from the Upper Cretaceous of France. Journal of Vertebrate Paleontology, 20:404-407. https:// doi.org/10.1671/0272-4634(2000)020[0404:angodd]2.0.co;2

Alonso, A., Canudo, J.I., Fernández-Baldor, F.T., and Huerta, P. 2017. Isolated theropod teeth associated with sauropod remains from El Oterillo II (Early Cretaceous) site of Salas de los Infantes (Burgos, Spain). Journal of Iberian Geology, 43:193-215. https://doi.org/10.1007/ s41513-017-0017-3

Alonso, A., Gasca, J.M., Navarro-Lorbés, P., Rubio, C., and Canudo, J.I. 2018. A new contribution to our knowledge of the large-bodied theropods from the Barremian of the Iberian Peninsula: the "Barranco del Hocino" site (Spain). Journal of Iberian Geology, 44:723. https://doi.org/10.1007/s41513-018-0051-9

Amiot, R., Buffetaut, E., Lécuyer, C., Fernandez, V., Fourel, F., Martineau, F., and Suteethorn, V. 2009. Oxygen isotope composition of continental vertebrate apatites from Mesozoic formations of Thailand; environmental and ecological significance. Geological Society, London, Special Publications, 315:271-283. https://doi.org/10.1144/SP315.19

Amiot, R., Buffetaut, E., Lécuyer, C., Wang, X., Boudad, L., Ding, Z., Fourel, F., Hutt, S., Martineau, F., Medeiros, M.A., Mo, J., Simon, L., Suteethorn, V., Sweetman, S., Tong, H., Zhang, F., and Zhou, Z. 2010. Oxygen isotope evidence for semi-aquatic habits among spinosaurid theropods. Geology, 38:139-142. https://doi.org/10.1130/G30402.1

Amiot, R., Lécuyer, C., Buffetaut, E., Escarguel, G., Fluteau, F., and Martineau, F. 2006. Oxygen isotopes from biogenic apatites suggest widespread endothermy in Cretaceous dinosaurs. Earth and Planetary Science Letters, 246:41-54. https://doi.org/10.1016/j.epsl.2006.04.018

Amiot, R., Lécuyer, C., Buffetaut, E., Fluteau, F., Legendre, S., and Martineau, F. 2004. Latitudinal temperature gradient during the Cretaceous Upper Campanian-Middle Maastrichtian: $\delta^{18} \mathrm{O}$ record of continental vertebrates. Earth and Planetary Science Letters, 226:255-272. https://doi.org/10.1016/j.epsl.2004.07.015

Amiot, R., Wang, X., Zhou, Z., Wang, X., Buffetaut, E., Lécuyer, C., Ding, Z., Fluteau, F., Hibino, T., Kusuhashi, N., Mo, J., Suteethorn, V., Wang, Y., Xu, X., and Zhang, F. 2011. Oxygen isotopes of East Asian dinosaurs reveal exceptionally cold Early Cretaceous climates. Proceedings of the National Academy of Sciences, 108:5179-5183.

Andres, B., Clark, J.M., and Xing, X. 2010. A new rhamphorhynchid pterosaur from the Upper Jurassic of Xinjiang, China, and the phylogenetic relationships of basal pterosaurs. Journal of Vertebrate Paleontology, 30:163-187. https://doi.org/10.1080/02724630903409220

Antunes, M.T. and Sigogneau-Russell, D. 1991. Nouvelles données sur les dinosaures du Crétacé supérieur du Portugal. Comptes Rendus de l'Académie des Sciences. Série 2, Mécanique, Physique, Chimie, Sciences de l'univers, Sciences de la Terre, 313:113-119.

Araújo, R., Castanhinha, R., Martins, R.M.S., Mateus, O., Hendrickx, C., Beckmann, F., Schell, N., and Alves, L.C. 2013. Filling the gaps of dinosaur eggshell phylogeny: Late Jurassic theropod clutch with embryos from Portugal. Scientific Reports, 3:1-8. https://doi.org/ 10.1038/srep01924

Arden, T.M.S., Klein, C.G., Zouhri, S., and Longrich, N.R. 2019. Aquatic adaptation in the skull of carnivorous dinosaurs (Theropoda: Spinosauridae) and the evolution of aquatic habits in spinosaurids. Cretaceous Research, 93:275-284. https://doi.org/10.1016/ j.cretres.2018.06.013

Auffenberg, W. 1981. The Behavioral Ecology of the Komodo Monitor. University Press of Florida, Gainesville.

Averianov, A. and Sues, H.-D. 2012. Skeletal remains of Tyrannosauroidea (Dinosauria: Theropoda) from the Bissekty Formation (Upper Cretaceous: Turonian) of Uzbekistan. Cretaceous Research, 34:284-297. https://doi.org/10.1016/j.cretres.2011.11.009

Averianov, A. and Sues, H.-D. 2016. Troodontidae (Dinosauria: Theropoda) from the Upper Cretaceous of Uzbekistan. Cretaceous Research, 59:98-110. https://doi.org/10.1016/ j.cretres.2015.11.005

Averianov, A.O., Ivantsov, S.V., and Skutschas, P.P. 2019. Theropod teeth from the Lower Cretaceous llek Formation of Western Siberia, Russia. Proceedings of the Zoological Institute RAS, 323:65-84. 
Averianov, A.O. and Sues, H.-D. 2007. A new troodontid (Dinosauria: Theropoda) from the Cenomanian of Uzbekistan, with a review of troodontid records from the territories of the former Soviet Union. Journal of Vertebrate Paleontology, 27:87-98. https://doi.org/10.1671/ 0272-4634(2007)27[87:antdtf]2.0.co;2

Avrahami, H.M., Gates, T.A., Heckert, A.B., Makovicky, P.J., and Zanno, L.E. 2018. A new microvertebrate assemblage from the Mussentuchit Member, Cedar Mountain Formation: insights into the paleobiodiversity and paleobiogeography of early Late Cretaceous ecosystems in western North America. PeerJ, 6:e5883. https://doi.org/10.7717/peerj.5883

Azuma, Y. and Currie, P.J. 2000. A new carnosaur (Dinosauria: Theropoda) from the Lower Cretaceous of Japan. Canadian Journal of Earth Sciences, 37:1735-1753. https://doi.org/ 10.1139/e00-064

Azuma, Y., Xu, X., Shibata, M., Kawabe, S., Miyata, K., and Imai, T. 2016. A bizarre theropod from the Early Cretaceous of Japan highlighting mosaic evolution among coelurosaurians. Scientific Reports, 6:20478. https://doi.org/10.1038/srep20478

Bakker, R.T. 1998. Brontosaur killers: Late Jurassic allosaurids as sabre-tooth cat analogues. Gaia, 15:145-158.

Bakker, R.T. and Bir, G. 2004. Dinosaur crime scene investigations: theropod behavior at Como Bluff, Wyoming, and the evolution of birdness, p. 301-342. In Currie, P.J., Koppelhus, E.B., Shugar, M.A., and Wright, J.L. (eds.), Feathered Dragons: Studies on the Transition from Dinosaurs to Birds. Indiana University Press, Bloomington, Indiana.

Bakker, R.T., Williams, M., and Currie, P.J. 1988. Nanotyrannus, a new genus of pygmy tyrannosaur, from the latest Cretaceous of Montana. Hunteria, 1:1-30.

Balanoff, A.M., Xu, X., Kobayashi, Y., Matsufune, Y., and Norell, M.A. 2009. Cranial osteology of the theropod dinosaur Incisivosaurus gauthieri (Theropoda: Oviraptorosauria). American Museum Novitates, 3651:1-35.

Baron, M.G. and Barrett, P.M. 2017. A dinosaur missing-link? Chilesaurus and the early evolution of ornithischian dinosaurs. Biology Letters, 13:20170220. https://doi.org/10.1098/ rsbl.2017.0220

Baron, M.G., Norman, D.B., and Barrett, P.M. 2017. A new hypothesis of dinosaur relationships and early dinosaur evolution. Nature, 543:501-506. https://doi.org/10.1038/nature21700

Barrett, P.M. 2000. Prosauropod dinosaurs and iguanas: speculations on the diets of extinct reptiles, p. 42-78. In Sues, H.-D. (ed.), Evolution of Herbivory in Terrestrial Vertebrates. Cambridge University Press, Cambridge, U.K.; New York.

Barrett, P.M. 2005. The diet of ostrich dinosaurs (Theropoda: Ornithomimosauria). Palaeontology, 48:347-358. https://doi.org/10.1111/j.1475-4983.2005.00448.x

Barrett, P.M. 2009. The affinities of the enigmatic dinosaur Eshanosaurus deguchiianus from the Early Jurassic of Yunnan Province, People's Republic of China. Palaeontology, 52:681-688. https://doi.org/10.1111/j.1475-4983.2009.00887.x

Barrett, P.M. and Rayfield, E.J. 2006. Ecological and evolutionary implications of dinosaur feeding behaviour. Trends in Ecology \& Evolution, 21:217-224. https://doi.org/10.1016/ j.tree.2006.01.002

Barrett, P.M. and Upchurch, P. 1994. Feeding mechanisms of Diplodocus. Gaia, 10:195-204.

Barsbold, R. 1974. Saurornithoididae, a new family of small theropod dinosaurs from central Asia and North America. Palaeontologia Polonica, 30:5-22.

Barsbold, R. and Osmólska, H. 1999. The skull of Velociraptor (Theropoda) from the Late Cretaceous of Mongolia. Acta Palaeontologica Polonica, 44:189-219.

Baszio, S. 1997. Systematic palaeontology of isolated dinosaur teeth from the latest Cretaceous of south Alberta, Canada. Courier Forschungsinstitut Senckenberg, 196:33-77.

Baumel, J.J. 1993. Handbook of Avian Anatomy: Nomina Anatomica Avium. 2nd ed. Publications of the Nuttall Ornithological Club, 23. Nuttall Ornithological Club, Cambridge.

Benson, R., Carrano, M., and Brusatte, S. 2010. A new clade of archaic large-bodied predatory dinosaurs (Theropoda: Allosauroidea) that survived to the latest Mesozoic. Naturwissenschaften, 97:71-78. https://doi.org/10.1007/s00114-009-0614-x

Benson, R.B.J. 2008. A redescription of 'Megalosaurus' hesperis (Dinosauria, Theropoda) from the Inferior Oolite (Bajocian, Middle Jurassic) of Dorset, United Kingdom. Zootaxa, 1931:5767. https://doi.org/10.11646/zootaxa.1931.1.5 
Benson, R.B.J. 2009. An assessment of variability in dinosaur remains from the Bathonian (Middle Jurassic) of Stonesfield and New Park Quarry, UK and taxonomic implications for Megalosaurus bucklandii and Iliosuchus incognitus. Palaeontology, 52:857-877. https:// doi.org/10.1111/j.1475-4983.2009.00884.x

Benson, R.B.J. 2010. A description of Megalosaurus bucklandii (Dinosauria: Theropoda) from the Bathonian of the UK and the relationships of Middle Jurassic theropods. Zoological Journal of the Linnean Society, 158:882-935.

Bertin, T. 2010. A catalogue of material and review of the Spinosauridae. PalArch's Journal of Vertebrate Palaeontology, 7:1-39.

Bhullar, B.-A.S., Hanson, M., Fabbri, M., Pritchard, A., Bever, G.S., and Hoffman, E. 2016. How to make a bird skull: major transitions in the evolution of the avian cranium, paedomorphosis, and the beak as a surrogate hand. Integrative and Comparative Biology, 56:389-403. https:// doi.org/10.1093/icb/icw069

Blob, R.W. and Badgley, C. 2007. Numerical methods for bonebed analysis, p. 333-396. In Rogers, R.R., Eberth, D.A., and Fiorillo, A.R. (eds.), Bonebeds: Genesis, Analysis, and Paleobiological Significance. University of Chicago Press, Chicago.

Bohlin, B. 1953. Fossil Reptiles from Mongolia and Kansu. Reports from the Scienitfic Expedition to the North-Western Provinces of China Under the Leadership of Dr Sven Hedin. The SinoSwedish Expedition. Publication 37. VI. Vertebrate Palaeontology. Statens Etnografiska Museum, Stockholm.

Bonde, N. and Leal, M.E.C. 2015. The detailed anatomy of Rhamphorhynchus II: braincase, pneumatics and jaws. Historical Biology, 27:755-770. https://doi.org/10.1080/ 08912963.2014.993630

Briggs, D.E.G. and Crowther, P.R. 2001. Palaeobiology II. Blackwell Science, Osney Mead, Oxford; Malden, MA.

Brink, K.S., Chen, Y.-C., Wu, Y.-N., Liu, W.-M., Shieh, D.-B., Huang, T.D., Sun, C.-K., and Reisz, R.R. 2016. Dietary adaptions in the ultrastructure of dinosaur dentine. Journal of the Royal Society Interface, 13:20160626. https://doi.org/10.1098/rsif.2016.0626

Brink, K.S., Reisz, R.R., LeBlanc, A.R.H., Chang, R.S., Lee, Y.C., Chiang, C.C., Huang, T., and Evans, D.C. 2015. Developmental and evolutionary novelty in the serrated teeth of theropod dinosaurs. Scientific Reports, 5:1-12. https://doi.org/10.1038/srep12338

Brinkman, D.B. 2008. The structure of Late Cretaceous (Late Campanian) nonmarine aquatic communities: a guild analysis of two vertebrate microfossil localities in Dinosaur Provincial Park, Alberta, Canada, p. 33-60. In Sankey, J.T. and Baszio, S. (eds.), Vertebrate Microfossil Assemblages: Their Role in Paleoecology and Paleobiogeography. Indiana University Press, Bloomington, Indiana.

Brown, D.S. and Cruickshank, A.R. 1994. The skull of the Callovian plesiosaur Cryptoclidus eurymerus, and the sauropterygian cheek. Palaeontology, 37:941-953.

Brusatte, S.L. 2012. Dinosaur Paleobiology. Wiley-Blackwell, Hoboken.

Brusatte, S.L., Averianov, A., Sues, H.-D., Muir, A., and Butler, I.B. 2016. New tyrannosaur from the mid-Cretaceous of Uzbekistan clarifies evolution of giant body sizes and advanced senses in tyrant dinosaurs. Proceedings of the National Academy of Sciences, 113:34473452. https://doi.org/10.1073/pnas.1600140113

Brusatte, S.L., Benson, R.B.J., Carr, T.D., Williamson, T.E., and Sereno, P.C. 2007. The systematic utility of theropod enamel wrinkles. Journal of Vertebrate Paleontology, 27:10521056. https://doi.org/10.1671/0272-4634(2007)27[1052:tsuote]2.0.co;2

Brusatte, S.L., Benson, R.B.J., Currie, P.J., and Xijin, Z. 2010a. The skull of Monolophosaurus jiangi (Dinosauria: Theropoda) and its implications for early theropod phylogeny and evolution. Zoological Journal of the Linnean Society, 158:573-607. https://doi.org/10.1111/ j.1096-3642.2009.00563.x

Brusatte, S.L., Benson, R.B.J., and Hutt, S. 2008. The osteology of Neovenator salerii (Dinosauria: Theropoda) from the Wealden Group (Barremian) of the Isle of Wight. Palaeontographical Society, 162:1-75.

Brusatte, S.L., Benson, R.B.J., and Norell, M.A. 2011. The anatomy of Dryptosaurus aquilunguis (Dinosauria: Theropoda) and a review of its tyrannosauroid affinities. American Museum Novitates, 3717:1-53. https://doi.org/10.1206/3717.2

Brusatte, S.L., Butler, R.J., Sulej, T., and Niedźwiedzki, G. 2009a. The taxonomy and anatomy of rauisuchian archosaurs from the Late Triassic of Germany and Poland. Acta Palaeontologica Polonica, 54:221-230. https://doi.org/10.4202/app.2008.0065 
Brusatte, S.L. and Carr, T.D. 2016. The phylogeny and evolutionary history of tyrannosauroid dinosaurs. Scientific Reports, 6:20252. https://doi.org/10.1038/srep20252

Brusatte, S.L., Carr, T.D., Erickson, G.M., Bever, G.S., and Norell, M.A. 2009b. A long-snouted, multihorned tyrannosaurid from the Late Cretaceous of Mongolia. Proceedings of the National Academy of Sciences, 106:17261-17266. https://doi.org/10.1073/pnas.0906911106

Brusatte, S.L., Carr, T.D., and Norell, M.A. 2012. The osteology of Alioramus, a gracile and longsnouted tyrannosaurid (Dinosauria: Theropoda) from the Late Cretaceous of Mongolia. Bulletin of the American Museum of Natural History, 366:1-197. https://doi.org/10.1206/770.1

Brusatte, S.L. and Clark, N.D.L. 2015. Theropod dinosaurs from the Middle Jurassic (BajocianBathonian) of Skye, Scotland. Scottish Journal of Geology, 2014-022. https://doi.org/10.1144/ sjg2014-022

Brusatte, S.L., Lloyd, G.T., Wang, S.C., and Norell, M.A. 2014. Gradual assembly of avian body plan culminated in rapid rates of evolution across the dinosaur-bird transition. Current Biology, 24:2386-2392. https://doi.org/10.1016/j.cub.2014.08.034

Brusatte, S.L., Norell, M.A., Carr, T.D., Erickson, G.M., Hutchinson, J.R., Balanoff, A.M., Bever, G.S., Choiniere, J.N., Makovicky, P.J., and Xu, X. 2010b. Tyrannosaur paleobiology: new research on ancient exemplar organisms. Science, 329:1481-1485. https://doi.org/10.1126/ science. 1193304

Brusatte, S.L. and Sereno, P.C. 2007. A new species of Carcharodontosaurus (Dinosauria: Theropoda) from the Cenomanian of Niger and a revision of the genus. Journal of Vertebrate Paleontology, 27:902-916. https://doi.org/10.1671/0272-4634(2007)27[902:ansocd]2.0.co;2

Brusatte, S.L. and Sereno, P.C. 2008. Phylogeny of Allosauroidea (Dinosauria: Theropoda): comparative analysis and resolution. Journal of Systematic Palaeontology, 6:155-182. https:/ /doi.org/10.1017/s1477201907002404

Buckley, L.G. 2009. Individual and Ontogenetic Variation in Theropod Dinosaur Teeth: a Case Study of Coelophysis bauri (Theropoda: Coelophysoidea) and Implications for Identifying Isolated Theropod Teeth. MSc. Dissertation, University of Alberta, Edmonton, Alberta, Canada.

Buckley, L.G. and Currie, P.J. 2014. Analysis of intraspecific and ontogenetic variation in the dentition of Coelophysis bauri (Late Triassic), and implications for the systematics of isolated theropod teeth. New Mexico Museum of Natural History and Science, 63:1-73.

Buckley, L.G., Larson, D.W., Reichel, M., and Samman, T. 2010. Quantifying tooth variation within a single population of Albertosaurus sarcophagus (Theropoda: Tyrannosauridae) and implications for identifying isolated teeth of tyrannosaurids. Canadian Journal of Earth Sciences, 47:1227-1251.

Buffetaut, E. 2011. An early spinosaurid dinosaur from the Late Jurassic of Tendaguru (Tanzania) and the evolution of the spinosaurid dentition. Oryctos, 10:1-8.

Buffetaut, E. and Bardet, N. 2012. The mosasaurid (Squamata) Prognathodon in the Maastrichtian (Late Cretaceous) of the Cotentin Peninsula (Normandy, northwestern France). Bulletin de la Société Géologique de France, 183:111-115. https://doi.org/10.2113/ gssgfbull.183.2.111

Buffetaut, E., Escuillié, F., and Pohl, B. 2005. First theropod dinosaur from the Maastrichtian phosphates of Morocco. Kaupia, 14:3-8.

Buffetaut, E. and Ingavat, R. 1986. Unusual theropod dinosaur teeth from the Upper Jurassic of Phu Wiang, northeastern Thailand. Revue de Paléobiologie, 5:217-220.

Burnham, D.A. 2004. New Information on Bambiraptor feinbergi (Theropoda: Dromaeosauridae) from the Late Cretaceous of Montana, p. 67-111. In Currie, P.J., Koppelhus, E.B., Shugar, M.A., and Wright, J.L. (eds.), Feathered Dragons: Studies on the Transition from Dinosaurs to Birds. Indiana University Press, Bloomington, Indiana.

Buscalioni, A.D., Gasparini, Z., Pérez-Moreno, B.P., and Sanz, J.L. 1996. Argentinean theropods: first morphological analysis on isolated teeth. Proceedings from the First European Workshop on Vertebrate Palaeontology. Geological Museum, Copenhagen University, 1-4 May 1996. Copenhagen. Pages unknown.

Button, K., You, H., Kirkland, J.I., and Zanno, L. 2017. Incremental growth of therizinosaurian dental tissues: implications for dietary transitions in Theropoda. PeerJ, 5:e4129. https:// doi.org/10.7717/peerj.4129

Cabreira, S.F., Kellner, A.W.A., Dias-da-Silva, S., Silva, L.R. da, Bronzati, M., Marsola, J.C. de A., Müller, R.T., Bittencourt, J. de S., Batista, B.J., Raugust, T., Carrilho, R., Brodt, A., and Langer, M.C. 2016. A unique Late Triassic dinosauromorph assemblage reveals dinosaur 
ancestral anatomy and diet. Current Biology, 26:3090-3095. https://doi.org/10.1016/ j.cub.2016.09.040

Canale, J.I., Novas, F.E., and Pol, D. 2015. Osteology and phylogenetic relationships of Tyrannotitan chubutensis Novas, de Valais, Vickers-Rich and Rich, 2005 (Theropoda: Carcharodontosauridae) from the Lower Cretaceous of Patagonia, Argentina. Historical Biology, 27:1-32. https://doi.org/10.1080/08912963.2013.861830

Canale, J.I., Scanferla, C.A., Agnolín, F.L., and Novas, F.E. 2009. New carnivorous dinosaur from the Late Cretaceous of NW Patagonia and the evolution of abelisaurid theropods. Naturwissenschaften, 96:409-414. https://doi.org/10.1007/s00114-008-0487-4

Candeiro, C.R.A. 2007. Padrões morfológicos dos dentes de Abelisauroidea y Carcharodontosauridae (Theropoda, Dinosauria) do Cretáceo da América do Sul. Ph.D. Dissertation, Universidade Federal do Rio de Janeiro, Departamento de Geología, Rio de Janeiro, Brazil.

Candeiro, C.R.A. and Tanke, D.H. 2008. A pathological Late Cretaceous carcharodontosaurid tooth from Minas Gerais, Brazil. Bulletin of Geosciences, 83:351-354.

Canudo, J.I., Gasulla, J.M., Gómez-Fernández, D., Ortega, F., Sanz, J.L., and Yagüe, P. 2008. Primera evidencia de dientes aislados atribuidos a Spinosauridae (Theropoda) en el Aptiano inferior (Cretácico Inferior) de Europa: Formación Arcillas de Morella (España). Ameghiniana, 45:649-662.

Carpenter, K. 1982. Baby dinosaurs from the Late Cretaceous Lance and Hell Creek formations and a description of a new species of theropod. Contributions to Geology, University of Wyoming, 20:123-134.

Carpenter, K. 1997. A giant coelophysoid (Ceratosauria) theropod from the Upper Triassic of New Mexico, USA. Neues Jahrbuch für Geologie und Paläontologie-Abhandlungen, 205:189-208.

Carr, T.D. 1999. Craniofacial ontogeny in Tyrannosauridae (Dinosauria, Coelurosauria). Journal of Vertebrate Paleontology, 19:497-520. https://doi.org/10.1080/02724634.1999.10011161

Carr, T.D. and Williamson, T.E. 2000. A review of Tyrannosauridae (Dinosauria, Coelurosauria) from New Mexico. New Mexico Museum of Natural History and Science Bulletin, 17:113-145.

Carr, T.D. and Williamson, T.E. 2004. Diversity of late Maastrichtian Tyrannosauridae (Dinosauria: Theropoda) from western North America. Zoological Journal of the Linnean Society, 142:479-523. https://doi.org/10.1111/j.1096-3642.2004.00130.x

Carr, T.D., Williamson, T.E., and Schwimmer, D.R. 2005. A new genus and species of tyrannosauroid from the Late Cretaceous (Middle Campanian) Demopolis Formation of Alabama. Journal of Vertebrate Paleontology, 25:119-143. https://doi.org/10.1671/02724634(2005)025[0119:angaso]2.0.co;2

Carrano, M.T., Benson, R.B.J., and Sampson, S.D. 2012. The phylogeny of Tetanurae (Dinosauria: Theropoda). Journal of Systematic Palaeontology, 10:211-300. https://doi.org/ 10.1080/14772019.2011.630927

Carrano, M.T., Loewen, M.A., and Sertich, J.J.W. 2011. New materials of Masiakasaurus knopfleri Sampson, Carrano, and Forster, 2001, and implications for the morphology of the Noasauridae (Theropoda: Ceratosauria). Smithsonian Contributions to Paleobiology, 95:153. https://doi.org/10.5479/si.00810266.95.1

Carrano, M.T., Sampson, S.D., and Forster, C.A. 2002. The osteology of Masiakasaurus knopfleri, a small abelisauroid (Dinosauria: Theropoda) from the Late Cretaceous of Madagascar. Journal of Vertebrate Paleontology, 22:510-534. https://doi.org/10.1671/02724634(2002)022[0510:toomka]2.0.co;2

Casal, G., Candeiro, C.R.A., Martínez, R., Ivany, E., and Ibiricu, L. 2009. Dientes de Theropoda (Dinosauria: Saurischia) de la Formación Bajo Barreal, Cretácico Superior, Provincia del Chubut, Argentina. Geobios, 42:553-560. https://doi.org/10.1016/j.geobios.2009.03.002.

Cau, A., Beyrand, V., Voeten, D.F.A.E., Fernandez, V., Tafforeau, P., Stein, K., Barsbold, R., Tsogtbaatar, K., Currie, P.J., and Godefroit, P. 2017. Synchrotron scanning reveals amphibious ecomorphology in a new clade of bird-like dinosaurs. Nature, 552:395-399. https://doi.org/10.1038/nature24679

Cau, A., Brougham, T., and Naish, D. 2015. The phylogenetic affinities of the bizarre Late Cretaceous Romanian theropod Balaur bondoc (Dinosauria, Maniraptora): dromaeosaurid or flightless bird? PeerJ, 3:e1032. https://doi.org/10.7717/peerj.1032 
Cau, A., Dyke, G.J., Lee, M.S.Y., and Naish, D. 2014. Data from: Sustained miniaturization and anatomical innovation in the dinosaurian ancestors of birds, downloaded 1 August 2014, Dryad Digital Repository.

Charig, A.J. and Milner, A.C. 1997. Baryonyx walkeri, a fish-eating dinosaur from the Wealden of Surrey. Bulletin of the Natural History Museum, 53:11-70.

Chiappe, L.M. and Göhlich, U.B. 2010. Anatomy of Juravenator starki (Theropoda: Coelurosauria) from the Late Jurassic of Germany. Neues Jahrbuch für Geologie und Paläontologie Abhandlungen, 258:257-296. https://doi.org/10.1127/0077-7749/2010/0125

Chiappe, L.M., Norell, M.A., and Clark, J.M. 1996. Phylogenetic position of Mononykus (Aves: Alvarezsauridae) from the Late Cretaceous of the Gobi Desert. Memoirs of the Queensland Museum, 39:557-582.

Chiappe, L.M., Norell, M.A., and Clark, J.M. 1998. The skull of a relative of the stem-group bird Mononykus. Nature, 392:275-278. https://doi.org/10.1038/32642

Chiappe, L.M. and Witmer, L.M. 2002. Mesozoic Birds: Above the Heads of Dinosaurs. University of California Press, Oakland.

Choiniere, J.N., Clark, J.M., Forster, C.A., Norell, M.A., Eberth, D.A., Erickson, G.M., Chu, H., and $\mathrm{Xu}, \mathrm{X}$. 2014a. A juvenile specimen of a new coelurosaur (Dinosauria: Theropoda) from the Middle-Late Jurassic Shishugou Formation of Xinjiang, People's Republic of China. Journal of Systematic Palaeontology, 12:177-215. https://doi.org/10.1080/ 14772019.2013.781067

Choiniere, J.N., Clark, J.M., Forster, C.A., and Xu, X. 2010a. A basal coelurosaur (Dinosauria: Theropoda) from the Late Jurassic (Oxfordian) of the Shishugou Formation in Wucaiwan, People's Republic of China. Journal of Vertebrate Paleontology, 30:1773-1796. https:// doi.org/10.1080/02724634.2010.520779

Choiniere, J.N., Clark, J.M., Norell, M., and Xu, X. 2014b. Cranial osteology of Haplocheirus sollers Choiniere et al., 2010 (Theropoda, Alvarezsauroidea). American Museum Novitates, 3816:1-44.

Choiniere, J.N., Forster, C.A., and de Klerk, W.J. 2012. New information on Nqwebasaurus thwazi, a coelurosaurian theropod from the Early Cretaceous Kirkwood Formation in South Africa. Journal of African Earth Sciences, 71-72:1-17. https://doi.org/10.1016/ j.jafrearsci.2012.05.005

Choiniere, J.N., Xu, X., Clark, J.M., Forster, C.A., Guo, Y., and Han, F. 2010b. A basal alvarezsauroid theropod from the Early Late Jurassic of Xinjiang, China. Science, 327:571574. https://doi.org/10.1126/science.1182143

Chure, D.J., Manabe, M., Tanimoto, M., and Tomida, Y. 1999. An unusual theropod tooth from the Mifune Group (late Cenomanian to early Turonian), Kumamoto, Japan. National Science Museum Monographs, 15:291-296.

Cillari, A. 2010. Teeth of Theropoda (Dinosauria, Saurischia): Morphology, Function and Classification. Ph.D. Dissertation, Scienze della Terra, Sapienza Universitá di Roma, Rome.

Clark, J.M., Maryanska, T., and Barsbold, R. 2004. Therizinosauroidea, p. 151-164. In Weishampel, D.B., Dodson, P., and Osmólska, H. (eds.), The Dinosauria, Second Edition. University of California Press, Berkeley, California.

Clark, J.M., Perle, A., and Norell, M. 1994. The skull of Erlicosaurus andrewsi, a Late Cretaceous "Segnosaur" (Theropoda, Therizinosauridae) from Mongolia. American Museum Novitates, 3115:1-39.

Cobos, A., Lockley, M.G., Gascó, F., Royo-Torres, R., and Alcalá, L. 2014. Megatheropods as apex predators in the typically Jurassic ecosystems of the Villar del Arzobispo Formation (Iberian Range, Spain). Palaeogeography, Palaeoclimatology, Palaeoecology, 399:31-41. https://doi.org/10.1016/j.palaeo.2014.02.008

Colbert, E.H. 1989. The Triassic dinosaur Coelophysis. Museum of Northern Arizona Bulletin, 57:1-174.

Cope, E.D. 1876a. On some extinct reptiles and Batrachia from the Judith River and Fox Hills Beds of Montana. Proceedings of the Academy of Natural Sciences of Philadelphia, 28:340359.

Cope, E.D. 1876b. Descriptions of some vertebrate remains from the Fort Union beds of Montana. Proceedings of the Academy of Natural Sciences of Philadelphia, 28:248-261.

Coria, R.A. and Currie, P.J. 2006. A new carcharodontosaurid (Dinosauria, Theropoda) from the Upper Cretaceous of Argentina. Geodiversitas, 28:71-118. 
Coria, R.A. and Currie, P.J. 2016. A new megaraptoran dinosaur (Dinosauria, Theropoda, Megaraptoridae) from the Late Cretaceous of Patagonia. PLoS ONE, 11:e0157973. https:// doi.org/10.1371/journal.pone.0157973

Cracraft, J. 1986. The origin and early diversification of birds. Paleobiology, 12:383-399.

Csiki-Sava, Z., Brusatte, S.L., and Vasile, Ş. 2016. "Megalosaurus cf. superbus" from southeastern Romania: The oldest known Cretaceous carcharodontosaurid (Dinosauria: Theropoda) and its implications for earliest Cretaceous Europe-Gondwana connections. Cretaceous Research, 60:221-238. https://doi.org/10.1016/j.cretres.2015.12.004

Cuesta, E., Vidal, D., Ortega, F., and Sanz, J.L. 2018. The cranial osteology of Concavenator corcovatus (Theropoda; Carcharodontosauria) from the Lower Cretaceous of Spain. Cretaceous Research, 91:176-194. https://doi.org/10.1016/j.cretres.2018.06.007

Currie, P.J. 1987. Bird-like characteristics of the jaws and teeth of troodontid theropods (Dinosauria, Saurischia). Journal of Vertebrate Paleontology, 7:72-81.

Currie, P.J. 1995. New information on the anatomy and relationships of Dromaeosaurus albertensis (Dinosauria: Theropoda). Journal of Vertebrate Paleontology, 15:576-591.

Currie, P.J. 2003. Cranial anatomy of tyrannosaurid dinosaurs from the Late Cretaceous of Alberta, Canada. Acta Palaeontologica Polonica, 48:191-226.

Currie, P.J. and Azuma, Y. 2006. New specimens, including a growth series, of Fukuiraptor (Dinosauria, Theropoda) from the Lower Cretaceous Kitadani Quarry of Japan. Journal of the Paleontological Society of Korea, 22:173-193

Currie, P.J. and Carabajal, A.P. 2012. A new specimen of Austroraptor cabazai Novas, Pol, Canale, Porfiri and Calvo, 2008 (Dinosauria, Theropoda, Unenlagiidae) from the Latest Cretaceous (Maastrichtian) of Río Negro, Argentina. Ameghiniana, 49:662-667. https:// doi.org/10.5710/AMGH.30.8.2012.574

Currie, P.J. and Carpenter, K. 2000. A new specimen of Acrocanthosaurus atokensis (Theropoda, Dinosauria) from the Lower Cretaceous Antlers Formation (Lower Cretaceous, Aptian) of Oklahoma, USA. Geodiversitas, 22:207-246.

Currie, P.J. and Chen, P. 2001. Anatomy of Sinosauropteryx prima from Liaoning, northeastern China. Canadian Journal of Earth Sciences, 38:1705-1727. https://doi.org/10.1139/e01-050.

Currie, P.J. and Dong, Z. 2001a. New information on Shanshanosaurus huoyanshanensis, a juvenile tyrannosaurid (Theropoda, Dinosauria) from the Late Cretaceous of China. Canadian Journal of Earth Sciences, 38:1729-1737. https://doi.org/10.1139/e01-042

Currie, P.J. and Dong, Z. 2001b. New information on Cretaceous troodontids (Dinosauria, Theropoda) from the People's Republic of China. Canadian Journal of Earth Sciences, 38:1753-1766. https://doi.org/10.1139/e01-065

Currie, P.J., Rigby, J.K., Jr., and Sloan, R.E. 1990. Theropod teeth from the Judith River Formation of southern Alberta, Canada, p. 107-125. In Carpenter, K. and Currie, P.J. (eds.), Dinosaur Systematics: Approaches and Perspectives. Cambridge University Press, New York.

Currie, P.J. and Varricchio, D.J. 2004. A new dromaeosaurid from the Horseshoe Canyon Formation (Upper Cretaceous) of Alberta, Canada, p. 112-132. In Currie, P.J., Koppelhus, E.B., Shugar, M.A., and Wright, J.L. (eds.), Feathered Dragons: Studies on the Transition from Dinosaurs to Birds. Indiana University Press, Bloomington, Indiana.

Currie, P.J. and Zhao, X.-J. 1993. A new carnosaur (Dinosauria, Theropoda) from the Jurassic of Xinjiang, People's Republic of China. Canadian Journal of Earth Sciences, 30:2037-2081. https://doi.org/10.1139/e93-179

Czerkas, S.A. and Yuan, C. 2002. An arboreal maniraptoran from northeast China. The Dinosaur Museum Journal, The Dinosaur Museum, Blanding, Utah, 1:63-95.

Dal Sasso, C. and Maganuco, S. 2011. Scipionyx samniticus (Theropoda: Compsognathidae) from the Lower Cretaceous of Italy: osteology, ontogenetic assessment, phylogeny, soft tissue anatomy, taphonomy and palaeobiology. Memorie della Società Italiana di Scienze Naturali e del Museo Civico di Storia Naturale di Milano, 37:1-281.

Dal Sasso, C., Maganuco, S., Buffetaut, E., and Mendez, M.A. 2005. New information on the skull of the enigmatic theropod Spinosaurus, with remarks on its size and affinities. Journal of Vertebrate Paleontology, 25:888-896. https://doi.org/10.1671/02724634(2005)025[0888:niotso]2.0.co;2

Dal Sasso, C., Maganuco, S., and Cau, A. 2018. The oldest ceratosaurian (Dinosauria: Theropoda), from the Lower Jurassic of Italy, sheds light on the evolution of the threefingered hand of birds. PeerJ, 6:e5976. https://doi.org/10.7717/peerj.5976 
D'Amore, D.C. 2009. A functional explanation for denticulation in theropod dinosaur teeth. The Anatomical Record: Advances in Integrative Anatomy and Evolutionary Biology, 292:12971314. https://doi.org/10.1002/ar.20977

D'Amore, D.C. and Blumenschine, R.J. 2009. Komodo monitor (Varanus komodoensis) feeding behavior and dental function reflected through tooth marks on bone surfaces, and the application to ziphodont paleobiology. Paleobiology, 35:525-552. https://doi.org/10.1666/ 0094-8373-35.4.525

D'Amore, D.C. and Blumenschine, R.J. 2012. Using striated tooth marks on bone to predict body size in theropod dinosaurs: a model based on feeding observations of Varanus komodoensis, the Komodo monitor. Paleobiology, 38:79-100. https://doi.org/10.1666/ 09079.1

Davit-Béal, T., Tucker, A.S., and Sire, J.-Y. 2009. Loss of teeth and enamel in tetrapods: fossil record, genetic data and morphological adaptations. Journal of Anatomy, 214:477-501. https://doi.org/10.1111/j.1469-7580.2009.01060.x

De Andrade, M., Young, M.T., Desojo, J.B., and Brusatte, S.L. 2010. The evolution of extreme hypercarnivory in Metriorhynchidae (Mesoeucrocodylia: Thalattosuchia) based on evidence from microscopic denticle morphology. Journal of Vertebrate Paleontology, 30:1451-1465. https://doi.org/10.1080/02724634.2010.501442

De Klerk, W.J., Forster, C.A., Sampson, S.D., Chinsamy, A., and Ross, C.F. 2000. A new coelurosaurian dinosaur from the Early Cretaceous of South Africa. Journal of Vertebrate Paleontology, 20:324-332. https://doi.org/10.1671/0272-4634(2000)020[0324:ancdft]2.0.co;2

Delcourt, R. and Grillo, O.N. 2018. Tyrannosauroids from the Southern Hemisphere: Implications for biogeography, evolution, and taxonomy. Palaeogeography, Palaeoclimatology, Palaeoecology, 511:379-387. https://doi.org/10.1016/j.palaeo.2018.09.003

DePalma, R.A., Burnham, D.A., Martin, L.D., Larson, P.L., and Bakker, R.T. 2015. The first giant raptor (Theropoda: Dromaeosauridae) from the Hell Creek Formation. Paleontological Contributions, 2015:1-16. https://doi.org/10.17161/paleo.1808.18764

Dong, Z. and Chen, P. 2000. A tiny fossil lizard in the stomach content of the feathered dinosaur Sinosauropteryx from northeastern China. Shi, L. and Zhang, F. (eds.), 5th International Meeting of the Society of Avian Paleontology and Evolution and the Symposium on Jehol Biota. Vertebrata PalAsiatica, 38(supplement): 10.

Dong, Z., Zhou, S.W., and Zhang, Y. 1983. Dinosaurs from the Jurassic of Sichuan. Palaeontologica Sinica, New Series C, 162:1-136.

Dufeau, D.L. 2003. The Cranial Anatomy of the Theropod Dinosaur Shuvuuia deserti (Coelurosauria: Alvarezsauridae), and its Bearing upon Coelurosaurian Phylogeny. MSc. Dissertation, University of Texas, Austin, Texas.

Eddy, D.R. and Clarke, J.A. 2011. New information on the cranial anatomy of Acrocanthosaurus atokensis and its implications for the phylogeny of Allosauroidea (Dinosauria: Theropoda). PLoS ONE, 6:e17932. https://doi.org/10.1371/journal.pone.0017932

Elzanowski, A. and Wellnhofer, P. 1993. Skull of Archaeornithoides from the Upper Cretaceous of Mongolia. American Journal of Science, 293:235-252. https://doi.org/10.2475/ ajs.293.A.235

Elzanowski, A. and Wellnhofer, P. 1996. Cranial morphology of Archaeopteryx: evidence from the seventh skeleton. Journal of Vertebrate Paleontology, 16:81-94. https://doi.org/10.1080/ 02724634.1996.10011286

Erickson, G.M. 1995. Split carinae on tyrannosaurid teeth and implications of their development. Journal of Vertebrate Paleontology, 15:268-274. https://doi.org/10.1080/ 02724634.1995.10011229

Erickson, G.M. 1996. Incremental lines of von Ebner in dinosaurs and the assessment of tooth replacement rates using growth line counts. Proceedings of the National Academy of Sciences, 93:14623-14627. https://doi.org/10.1073/pnas.93.25.14623

Erickson, G.M., Zelenitsky, D.K., Kay, D.I., and Norell, M.A. 2017. Dinosaur incubation periods directly determined from growth-line counts in embryonic teeth show reptilian-grade development. Proceedings of the National Academy of Sciences, 114:540-545. https:// doi.org/10.1073/pnas.1613716114

Evans, D.C., Larson, D.W., and Currie, P.J. 2013. A new dromaeosaurid (Dinosauria: Theropoda) with Asian affinities from the latest Cretaceous of North America. Naturwissenschaften, 100:1041-1049. https://doi.org/10.1007/s00114-013-1107-5 
Ezcurra, M. 2017. A new early coelophysoid neotheropod from the Late Triassic of Northwestern Argentina. Ameghiniana, 54:506-538. https://doi.org/10.5710/amgh.04.08.2017.3100

Ezcurra, M.D. 2007. The cranial anatomy of the coelophysoid theropod Zupaysaurus rougieri from the Upper Triassic of Argentina. Historical Biology, 19:185-202. https://doi.org/10.1080/ 08912960600861467

Ezcurra, M.D. 2009. Theropod remains from the uppermost Cretaceous of Colombia and their implications for the palaeozoogeography of western Gondwana. Cretaceous Research, 30:1339-1344. https://doi.org/10.1016/j.cretres.2009.08.004

Fanti, F., Cau, A., Martinelli, A., and Contessi, M. 2014. Integrating palaeoecology and morphology in theropod diversity estimation: A case from the Aptian-Albian of Tunisia. Palaeogeography, Palaeoclimatology, Palaeoecology, 410:39-57. https://doi.org/10.1016/ j.palaeo.2014.05.033

Fanti, F. and Therrien, F. 2007. Theropod tooth assemblages from the Late Cretaceous Maevarano Formation and the possible presence of dromaeosaurids in Madagascar. Acta Palaeontologica Polonica, 52:155-166

Farlow, J.O., Brinkman, D.L., Abler, W.L., and Currie, P.J. 1991. Size, shape, and serration density of theropod dinosaur lateral teeth. Modern Geology, 16:161-198.

Farris, J.S. 1989. The retention index and the rescaled consistency index. Cladistics, 5:417-419. https://doi.org/10.1111/j.1096-0031.1989.tb00573.x

Fiorillo, A.R. and Currie, P.J. 1994. Theropod teeth from the Judith River Formation (Upper Cretaceous) of south-central Montana. Journal of Vertebrate Paleontology, 14:74-80. https:// doi.org/10.1080/02724634.1994.10011539

Fiorillo, A.R. and Gangloff, R.A. 2001. Theropod teeth from the Prince Creek Formation (Cretaceous) of northern Alaska, with speculations on Arctic dinosaur paleoecology. Journal of Vertebrate Paleontology, 20:675-682. https://doi.org/10.1671/02724634(2000)020[0675:ttftpc]2.0.co;2

Folinsbee, K.E., Müller, J., and Reisz, R.R. 2007. Canine grooves: morphology, function, and relevance to venom. Journal of Vertebrate Paleontology, 27:547-551. https://doi.org/10.1671/ 0272-4634(2007)27[547:cgmfar]2.0.co;2

Forster, C.A., Sampson, S.D., Chiappe, L.M., and Krause, D.W. 1998. The theropod ancestry of birds: new evidence from the Late Cretaceous of Madagascar. Science, 279:1915-1919. https://doi.org/10.1126/science.279.5358.1915

Foth, C. and Rauhut, O.W.M. 2017. Re-evaluation of the Haarlem Archaeopteryx and the radiation of maniraptoran theropod dinosaurs. BMC Evolutionary Biology, 17:236. https:// doi.org/10.1186/s12862-017-1076-y

Foth, C., Tischlinger, H., and Rauhut, O.W.M. 2014. New specimen of Archaeopteryx provides insights into the evolution of pennaceous feathers. Nature, 511:79-82. https://doi.org/ 10.1038/nature13467

Fowler, D.W., Freedman, E.A., Scannella, J.B., and Kambic, R.E. 2011a. The predatory ecology of Deinonychus and the origin of flapping in birds. PLOS ONE, 6:e28964. https://doi.org/ 10.1371/journal.pone.0028964

Fowler, D.W., Woodward, H.N., Freedman, E.A., Larson, P.L., and Horner, J.R. 2011b. Reanalysis of "Raptorex kriegsteini": a juvenile tyrannosaurid dinosaur from Mongolia. PLoS ONE, 6:e21376. https://doi.org/10.1371/journal.pone.0021376

Frederickson, J.A., Engel, M.H., and Cifelli, R.L. 2018. Niche partitioning in theropod dinosaurs: diet and habitat preference in predators from the Uppermost Cedar Mountain Formation (Utah, U.S.A.). Scientific Reports, 8:17872. https://doi.org/10.1038/s41598-018-35689-6

Freedman, L. 1957. The fossil Cercopithecoidea of South Africa. Annals of the Transvaal Museum, 23:121-262.

Fry, B.G., Wroe, S., Teeuwisse, W., Osch, M.J.P. van, Moreno, K., Ingle, J., McHenry, C., Ferrara, T., Clausen, P., Scheib, H., Winter, K.L., Greisman, L., Roelants, K., van der Weerd, L., Clemente, C.J., Giannakis, E., Hodgson, W.C., Luz, S., Martelli, P., Krishnasamy, K., Kochva, E., Kwok, H.F., Scanlon, D., Karas, J., Citron, D.M., Goldstein, E.J.C., Mcnaughtan, J.E., and Norman, J.A. 2009. A central role for venom in predation by Varanus komodoensis (Komodo Dragon) and the extinct giant Varanus (Megalania) priscus. Proceedings of the National Academy of Sciences, 106:8969-8974. https://doi.org/10.1073/pnas.0810883106 
Funston, G.F., Currie, P.J., Eberth, D.A., Ryan, M.J., Chinzorig, T., Badamgarav, D., and Longrich, N.R. 2016. The first oviraptorosaur (Dinosauria: Theropoda) bonebed: evidence of gregarious behaviour in a maniraptoran theropod. Scientific Reports, 6:35782. https://doi.org/ 10.1038/srep35782

Galton, P.M. 1984. Cranial anatomy of the prosauropod dinosaur Plateosaurus from the Knollenmergel (Middle Keuper, Upper Triassic) of Germany. I. Two complete skulls from Trossingen/Württ. with comments on the diet. Geologica et Palaeontologica, 18:139-171.

Galton, P.M. 1985. Diet of prosauropod dinosaurs from the late Triassic and early Jurassic. Lethaia, 18:105-123. https://doi.org/10.1111/j.1502-3931.1985.tb00690.x

Galton, P.M. and Molnar, R.E. 2012. An unusually large theropod dinosaur tooth from the Kirkwood Formation (Lower Cretaceous) of South Africa. Neues Jahrbuch für Geologie und Paläontologie - Abhandlungen, 263:17-23. https://doi.org/10.1127/0077-7749/2011/0204

Gao, C., Morschhauser, E.M., Varricchio, D.J., Liu, J., and Zhao, B. 2012. A second soundly sleeping dragon: new anatomical details of the Chinese troodontid Mei long with implications for phylogeny and taphonomy. PLoS ONE, 7:e45203. https://doi.org/10.1371/ journal.pone.0045203

Gauthier, J. 1986. Saurischian monophyly and the origin of birds, p. 1-55. In Padian, K. (ed.), The Origin of Birds and the Evolution of Flight. Memoirs of the California Academy of Sciences Number 8. California Academy of Sciences, San Francisco, California.

Gerke, O. and Wings, O. 2016. Multivariate and cladistic analyses of isolated teeth reveal sympatry of theropod dinosaurs in the Late Jurassic of Northern Germany. PLOS ONE, 11:e0158334. https://doi.org/10.1371/journal.pone.0158334

Gianechini, F.A., Agnolín, F.L., and Ezcurra, M.D. 2011a. A reassessment of the purported venom delivery system of the bird-like raptor Sinornithosaurus. Paläontologische Zeitschrift, 85:103-107. https://doi.org/10.1007/s12542-010-0074-9

Gianechini, F.A., Apesteguía, S., Landini, W., Finotti, F., Juárez Valieri, R., and Zandonai, F. 2015. New abelisaurid remains from the Anacleto Formation (Upper Cretaceous), Patagonia, Argentina. Cretaceous Research, 54:1-16. https://doi.org/10.1016/j.cretres.2014.11.009

Gianechini, F.A., Makovicky, P.J., and Apesteguía, S. 2011b. The teeth of the unenlagiine theropod Buitreraptor from the Cretaceous of Patagonia, Argentina, and the unusual dentition of the Gondwanan dromaeosaurids. Acta Palaeontologica Polonica, 56:279-290.

Gilmore, C.W. 1924. On Troodon validus, an ornithopodus dinosaur from the Belly River Cretaceous of Alberta, Canada. Bulletin of the Department of Geology, University of Alberta, 1:1-143.

Godefroit, P., Cau, A., Dong-Yu, H., Escuillié, F., Wenhao, W., and Dyke, G. 2013a. A Jurassic avialan dinosaur from China resolves the early phylogenetic history of birds. Nature, 498:359-362. https://doi.org/10.1038/nature12168

Godefroit, P., Currie, P.J., Hong, L., Yong, S.C., and Zhi-Ming, D. 2008. A new species of Velociraptor (Dinosauria: Dromaeosauridae) from the Upper Cretaceous of northern China. Journal of Vertebrate Paleontology, 28:432-438. https://doi.org/10.1671/02724634(2008)28[432:ansovd]2.0.co;2

Godefroit, P., Demuynck, H., Dyke, G., Hu, D., Escuillié, F., and Claeys, P. 2013b. Reduced plumage and flight ability of a new Jurassic paravian theropod from China. Nature Communications, 4:1394. https://doi.org/10.1038/ncomms2389

Goldstein, E.J.C., Tyrrell, K.L., Citron, D.M., Cox, C.R., Recchio, I.M., Okimoto, B., Bryja, J., and Fry, B.G. 2013. Anaerobic and aerobic bacteriology of the saliva and gingiva from 16 captive komodo dragons (Varanus komodoensis): new implications for the "bacteria as venom" model. Journal of Zoo and Wildlife Medicine, 44:262-272. https://doi.org/10.1638/20120022R.1

Goloboff, P.A. and Catalano, S.A. 2016. TNT version 1.5, including a full implementation of phylogenetic morphometrics. Cladistics, 32:221-238. https://doi.org/10.1111/cla.12160

Gong, E., Martin, L.D., Burnham, D.A., and Falk, A.R. 2010. The birdlike raptor Sinornithosaurus was venomous. Proceedings of the National Academy of Sciences, 107:766-768. https:// doi.org/10.1073/pnas.0912360107

Gong, E., Martin, L.D., Burnham, D.A., and Falk, A.R. 2011. Evidence for a venomous Sinornithosaurus. Paläontologische Zeitschrift, 85:109-111. https://doi.org/10.1007/s12542010-0076-7 
Goswami, A., Prasad, G.V.R., Verma, O., Flynn, J.J., and Benson, R.B.J. 2013. A troodontid dinosaur from the latest Cretaceous of India. Nature Communications, 4:1703. https:// doi.org/10.1038/ncomms 2716

Griffin, C.T. 2019. Large neotheropods from the Upper Triassic of North America and the early evolution of large theropod body sizes. Journal of Paleontology, 93:1010-1030. https:// doi.org/10.1017/jpa.2019.13

Hammer, Ø., Harper, D.A.T., and Ryan, P.D. 2001. PAST: Paleontological statistics software package for education and data analysis. Palaeontologia Electronica, 4.14A:1-9. https://palaeo-electronica.org/2001_1/past/issue1_01.htm

Han, F., Clark, J.M., Xu, X., Sullivan, C., Choiniere, J., and Hone, D.W.E. 2011. Theropod teeth from the Middle-Upper Jurassic Shishugou Formation of northwest Xinjiang, China. Journal of Vertebrate Paleontology, 31:111-126. https://doi.org/10.1080/02724634.2011.546291

Han, G., Chiappe, L.M., Ji, S.-A., Habib, M., Turner, A.H., Chinsamy, A., Liu, X., and Han, L. 2014. A new raptorial dinosaur with exceptionally long feathering provides insights into dromaeosaurid flight performance. Nature Communications, 5:1-9. https://doi.org/10.1038/ ncomms5382

Harris, J.D. 1998. A reanalysis of Acrocanthosaurus atokensis, its phylogenetic status, and paleobiogeographic implications, based on a new specimen from Texas. New Mexico Museum of Natural History and Science Bulletin, 13:1-75.

Harris, J.D. 2004. Confusing dinosaurs with mammals: Tetrapod phylogenetics and anatomical terminology in the world of homology. The Anatomical Record Part A: Discoveries in Molecular, Cellular, and Evolutionary Biology, 281A:1240-1246. https://doi.org/10.1002/ ar.a.20078

Hasegawa, Y., Tanaka, G., Takakuwa, Y., and Koike, S. 2010. Fine sculptures on a tooth of Spinosaurus (Dinosauria, Theropoda) from Morocco. Bulletin of Gunma Museum of Natural History, 14:11-20.

Hassler, A., Martin, J.E., Amiot, R., Tacail, T., Godet, F.A., Allain, R., and Balter, V. 2018. Calcium isotopes offer clues on resource partitioning among Cretaceous predatory dinosaurs. Proceedings of the Royal Society B, 285:20180197. https://doi.org/10.1098/rspb.2018.0197

Hedrick, B.P., Zanno, L.E., Wolfe, D.G., and Dodson, P. 2015. The slothful claw: osteology and taphonomy of Nothronychus mckinleyi and N. graffami (Dinosauria: Theropoda) and anatomical considerations for derived therizinosaurids. PLOS ONE, 10:e0129449. https:// doi.org/10.1371/journal.pone.0129449

Hendrickx, C. 2015. Evolution of Teeth and Quadrate in Non-avian Theropoda (Dinosauria: Saurischia), with the Description of Torvosaurus Remains from Portugal. Ph.D. Dissertation, Universidade Nova de Lisboa, Lisbon, Portugal.

Hendrickx, C., Araújo, R., and Mateus, O. 2015a. The non-avian theropod quadrate I: standardized terminology with an overview of the anatomy and function. PeerJ, 3:e1245. https://doi.org/10.7717/peerj.1245

Hendrickx, C. and Carrano, M.T. 2016. Erratum on "An overview of non-avian theropod discoveries and classification." PalArch's Journal of Vertebrate Palaeontology, 13:1-7.

Hendrickx, C., Hartman, S.A., and Mateus, O. 2015b. An overview of non-avian theropod discoveries and classification. PalArch's Journal of Vertebrate Palaeontology, 12:1-73.

Hendrickx, C. and Mateus, O. 2014a. Abelisauridae (Dinosauria: Theropoda) from the Late Jurassic of Portugal and dentition-based phylogeny as a contribution for the identification of isolated theropod teeth. Zootaxa, 3759:1-74. https://doi.org/10.11646/zootaxa.3759.1.1

Hendrickx, C. and Mateus, O. 2014b. Torvosaurus gurneyi n. sp., the largest terrestrial predator from Europe, and a proposed terminology of the maxilla anatomy in nonavian theropods. PLoS ONE, 9:e88905. https://doi.org/10.1371/journal.pone.0088905

Hendrickx, C., Mateus, O., and Araújo, R. 2015c. The dentition of megalosaurid theropods. Acta Palaeontologica Polonica, 60:627-642. https://doi.org/10.4202/app.00056.2013

Hendrickx, C., Mateus, O., and Araújo, R. 2015d. A proposed terminology of theropod teeth (Dinosauria, Saurischia). Journal of Vertebrate Paleontology, 35:e982797. https://doi.org/ 10.1080/02724634.2015.982797

Hendrickx, C., Mateus, O., and Buffetaut, E. 2016. Morphofunctional analysis of the quadrate of Spinosauridae (Dinosauria: Theropoda) and the presence of Spinosaurus and a second spinosaurine taxon in the Cenomanian of North Africa. PLOS ONE, 11:e0144695. https:// doi.org/10.1371/journal.pone.0144695

Hillson, S. 2005. Teeth: Second Edition. Cambridge University Press, New York. 
Hocknull, S.A., White, M.A., Tischler, T.R., Cook, A.G., Calleja, N.D., Sloan, T., and Elliott, D.A. 2009. New mid-Cretaceous (Latest Albian) dinosaurs from Winton, Queensland, Australia. PLOS ONE, 4:e6190. https://doi.org/10.1371/journal.pone.0006190

Holtz, T.R. 1994. The phylogenetic position of the Tyrannosauridae: implications for theropod systematics. Journal of Paleontology, 68:1100-1117.

Holtz, T.R.J. 1998a. Spinosaurs as crocodile mimics. Science, 282:1276-1277. https://doi.org/ 10.1126/science.282.5392.1276

Holtz, T.R.J. 1998b. A new phylogeny of the carnivorous dinosaurs. Gaia, 15:5-61.

Holtz, T.R.J. 2001. The phylogeny and taxonomy of the Tyrannosauridae, p. 64-83. In Tanke, D.H., Carpenter, K., and Skrepnick, M.W. (eds.), Mesozoic Vertebrate Life. Indiana University Press, Bloomington, Indiana.

Holtz, T.R.J. 2003. Dinosaur predation: evidence and ecomorphology, p. 325-340. In Kelley, P.H., Kowalewski, M., and Hansen, T.A. (eds.), Predator-Prey Interactions in the Fossil Record. Topics in Geobiology 20. Kluwer Academic/Plenum Publishers, New York, Boston, Dordrecht, London, Moscow.

Holtz, T.R.J. 2004. Tyrannosauroidea, p. 111-136. In Weishampel, D.B., Dodson, P., and Osmólska, H. (eds.), The Dinosauria. Second Edition. University of California Press, Berkeley, California.

Holtz, T.R.J. 2008. A critical reappraisal of the obligate scavenging hypothesis for Tyrannosaurus rex and other tyrant dinosaurs, p. 371-396. In Larson, P.L. and Kenneth, C. (eds.), Tyrannosaurus rex, the Tyrant King. Indiana University Press, Bloomington, Indiana.

Holtz, T.R.J., Brinkman, D.L., and Chandler, C.L. 1998. Denticle morphometrics and a possibly omnivorous feeding habit for the theropod dinosaur Troodon. Gaia, 15:159-166.

Holtz, T.R.J., Molnar, R.E., and Currie, P.J. 2004. Basal Tetanurae, p. 71-110. In Weishampel, D.B., Dodson, P., and Osmólska, H. (eds.), The Dinosauria. Second Edition. University of California Press, Berkeley, California.

Hone, D.W.E., Wang, K., Sullivan, C., Zhao, X., Chen, S., Li, D., Ji, S., Ji, Q., and Xu, X. 2011. A new, large tyrannosaurine theropod from the Upper Cretaceous of China. Cretaceous Research, 32:495. https://doi.org/10.1016/j.cretres.2011.03.005

Howgate, M.E. 1984. The teeth of Archaeopteryx and a reinterpretation of the Eichstätt specimen. Zoological Journal of the Linnean Society, 82:159-175. https://doi.org/10.1111/ j.1096-3642.1984.tb00540.x

Hu, D., Clarke, J.A., Eliason, C.M., Qiu, R., Li, Q., Shawkey, M.D., Zhao, C., D’Alba, L., Jiang, J., and Xu, X. 2018. A bony-crested Jurassic dinosaur with evidence of iridescent plumage highlights complexity in early paravian evolution. Nature Communications, 9:217. https:// doi.org/10.1038/s41467-017-02515-y

Hu, D., Hou, L., Zhang, L., and Xu, X. 2009. A pre-Archaeopteryx troodontid theropod from China with long feathers on the metatarsus. Nature, 461:640-643. https://doi.org/10.1038/ nature 08322

Hurum, J.H. and Sabath, K. 2003. Giant theropod dinosaurs from Asia and North America: Skulls of Tarbosaurus bataar and Tyrannosaurus rex compared. Acta Palaeontologica Polonica, 48:161-190.

Hutt, S., Naish, D., Martill, D.M., Barker, M.J., and Newbery, P. 2001. A preliminary account of a new tyrannosauroid theropod from the Wessex Formation (Early Cretaceous) of southern England. Cretaceous Research, 22:227-242. https://doi.org/10.1006/cres.2001.0252

Hwang, S.H. 2005. Phylogenetic patterns of enamel microstructure in dinosaur teeth. Journal of Morphology, 266:208-240. https://doi.org/10.1002/jmor.10372

Hwang, S.H. 2007. Phylogenetic Patterns of Enamel Microstructure in Dinosaur Teeth. Ph.D. Dissertation, Columbia University, New York, New York.

Hwang, S.H. 2010. The utility of tooth enamel microstructure in identifying isolated dinosaur teeth. Lethaia, 43:307-322. https://doi.org/10.1111/j.1502-3931.2009.00194.x

Hwang, S.H., Norell, M.A., Ji, Q., and Keqin, G. 2002. New specimens of Microraptor zhaoianus (Theropoda: Dromaeosauridae) from Northeastern China. American Museum Novitates, 3381:1-44. https://doi.org/10.1206/0003-0082(2002)381\%3C0001:nsomzt\%3E2.0.co;2

Hwang, S.H., Norell, M.A., Qiang, J., and Keqin, G. 2004. A large compsognathid from the Early Cretaceous Yixian Formation of China. Journal of Systematic Palaeontology, 2:13-30. https:// doi.org/10.1017/S1477201903001081 
Ibrahim, N., Sereno, P.C., Sasso, C.D., Maganuco, S., Fabbri, M., Martill, D.M., Zouhri, S., Myhrvold, N., and lurino, D.A. 2014. Semiaquatic adaptations in a giant predatory dinosaur. Science, 345:1613-1616. https://doi.org/10.1126/science.1258750

ICVGAN. 2012. Nomina Anatomica Veterinaria (Revised 5th edition). International Committee on Veterinary Gross Anatomical Nomenclature (ICVGAN). Union of International Associations, Brussels.

Jacobsen, A.R. 1997. Tooth marks, p. 738. In Currie, P.J. and Padian, K. (eds.), Encyclopedia of Dinosaurs. Academic Press, San Diego, California.

Janensch, W. 1925. Die Coelurosaurier und Theropoden der Tendaguruschichten DeutschOstafrikas. Palaeontographica-Supplementbände, 1:1-100.

Ji, Q., Currie, P.J., Norell, M.A., and Shu-An, J. 1998. Two feathered dinosaurs from northeastern China. Nature, 393:753-761. https://doi.org/10.1038/31635

Ji, Q., Ji, S., Lü, J., You, H., Chen, W., Liu, Y., and Liu, Y. 2005. First avialian bird from China. Geological Bulletin of China, 24:197-210.

Ji, Q., Ji, S., Lü, J., and Yuan, C. 2007. A new giant compsognathid dinosaur with long filamentous integuments from Lower Cretaceous of Northeastern China. Acta Geologica Sinica, 81:8-15.

Ji, Q., Norell, M.A., Makovicky, P.J., Gao, K.-Q., Ji, S., and Yuan, C. 2003. An early ostrich dinosaur and implications for ornithomimosaur phylogeny. American Museum Novitates, 3420:1-19. https://doi.org/10.1206/0003-0082(2003)420\%3C0001:aeodai\%3E2.0.co;2

Jin, L., Chen, J., and Godefroit, P. 2012. A new basal ornithomimosaur (Dinosauria: Theropoda) from the Early Cretaceous Yixian Formation, Northeast China, p. 466-487. In Godefroit, P. (ed.), Bernissart Dinosaurs and Early Cretaceous Terrestrial Ecosystems. Indiana University Press, Bloomington, Indiana.

Ji, S. and Ji, Q. 2007. Jinfengopteryx compared to Archaeopteryx, with comments on the mosaic evolution of long-tailed avialan birds. Acta Geologica Sinica, 81:337-343. https://doi.org/ 10.1111/j.1755-6724.2007.tb00957.x

Jouve, S. 2004. Description of the skull of a Ctenochasma (Pterosauria) from the latest Jurassic of eastern France, with a taxonomic revision of European Tithonian Pterodactyloidea. Journal of Vertebrate Paleontology, 24:542-554. https://doi.org/10.1671/02724634(2004)024[0542:dotsoa]2.0.co;2

Kear, B.P., Rich, T.H., Vickers-Rich, P., Ali, M.A., Al-Mufarreh, Y.A., Matari, A.H., Al-Massari, A.M., Nasser, A.H., Attia, Y., and Halawani, M.A. 2013. First dinosaurs from Saudi Arabia. PLoS ONE, 8:e84041. https://doi.org/10.1371/journal.pone.0084041

Kearney, M., Rieppel, O., and Wood, R.M. 2006. An investigation into the occurrence of plicidentine in the teeth of squamate reptiles. Copeia, 2006:337-350. https://doi.org/10.1643/ 0045-8511(2006)2006[337:aiitoo]2.0.co;2

Kellner, A.W.A. 1996. Remarks on Brazilian dinosaurs. Memoirs of the Queensland Museum, 39:611-626.

Kellner, A.W.A. and Campos, D.A. 1996. First Early Cretaceous theropod dinosaur from Brazil with comments on Spinosauridae. Neues Jahrbuch für Geologie und Paläontologie Abhandlungen, 199:151-166.

Kellner, A.W.A. and Tomida, Y. 2000. Description of a new species of Anhangueridae (Pterodactyloidea) with comments on the pterosaur fauna from the Santana Formation (Aptian-Albian), Northeastern Brazil. National Science Museum Monographs, 17:1-137.

Kirkland, J.I., Gaston, R., and Burge, D. 1993. A large dromaeosaur (Theropoda) from the Lower Cretaceous of eastern Utah. Hunteria, 2:1-16.

Kirkland, J.I., Simpson, E.L., DeBlieux, D.D., Madsen, S.K., Bogner, E., and Tibert, N.E. 2016. Depositional constraints on the Lower Cretaceous stikes quarry dinosaur site: Upper yellow cat member, cedar mountain formation, Utah. Palaios, 31:421-439. https://doi.org/10.2110/ palo.2016.041

Kirkland, J.I. and Wolfe, D.G. 2001. First definitive therizinosaurid (Dinosauria; Theropoda) from North America. Journal of Vertebrate Paleontology, 21:410-414. https://doi.org/10.1671/ 0272-4634(2001)021[0410:fdtdtf]2.0.co;2

Kirkland, J.I., Zanno, L.E., Sampson, S.D., Clark, J.M., and DeBlieux, D.D. 2005. A primitive therizinosauroid dinosaur from the Early Cretaceous of Utah. Nature, 435:84-87. https:// doi.org/10.1038/nature03468

Kitching, I.J., Forey, P.L., Humphries, C.J., and Williams, D.M. 1998. Cladistics: The Theory and Practice of Parsimony Analysis 2nd Edition. Oxford University Press, Oxford. 
Kluge, A.G. and Farris, J.S. 1969. Quantitative phyletics and the evolution of anurans. Systematic Biology, 18:1-32. https://doi.org/10.1093/sysbio/18.1.1

Kobayashi, Y. and Barsbold, R. 2005. Reexamination of a primitive ornithomimosaur, Garudimimus brevipes Barsbold, 1981 (Dinosauria: Theropoda), from the Late Cretaceous of Mongolia. Canadian Journal of Earth Sciences, 42:1501-1521. https://doi.org/10.1139/e05044

Kobayashi, Y., Lu, J.-C., Dong, Z.-M., Barsbold, R., Azuma, Y., and Tomida, Y. 1999. Herbivorous diet in an ornithomimid dinosaur. Nature, 402:480-481. https://doi.org/10.1038/ 44999

Kundrát, M., Nudds, J., Kear, B.P., Lü, J., and Ahlberg, P. 2019. The first specimen of Archaeopteryx from the Upper Jurassic Mörnsheim Formation of Germany. Historical Biology, 31:3-63. https://doi.org/10.1080/08912963.2018.1518443

Lamanna, M.C., Sues, H.-D., Schachner, E.R., and Lyson, T.R. 2014. A new large-bodied oviraptorosaurian theropod dinosaur from the Latest Cretaceous of Western North America. PLoS ONE, 9:e92022. https://doi.org/10.1371/journal.pone.0092022

Langer, M.C. and Benton, M.J. 2006. Early dinosaurs: a phylogenetic study. Journal of Systematic Palaeontology, 4:309-358. https://doi.org/10.1017/S1477201906001970

Langer, M.C., Ezcurra, M.D., Rauhut, O.W.M., Benton, M.J., Knoll, F., McPhee, B.W., Novas, F.E., Pol, D., and Brusatte, S.L. 2017. Untangling the dinosaur family tree. Nature, 551:E1E3. https://doi.org/10.1038/nature24011

Langer, M.C., Martins, N. de O., Manzig, P.C., Ferreira, G. de S., Marsola, J.C. de A., Fortes, E., Lima, R., Sant'ana, L.C.F., Vidal, L. da S., Lorençato, R.H. da S., and Ezcurra, M.D. 2019. A new desert-dwelling dinosaur (Theropoda, Noasaurinae) from the Cretaceous of south Brazil. Scientific Reports, 9:9379. https://doi.org/10.1038/s41598-019-45306-9

Lanser, K.-P. and Heimhofer, U. 2015. Evidence of theropod dinosaurs from a Lower Cretaceous karst filling in the northern Sauerland (Rhenish Massif, Germany). Paläontologische Zeitschrift, 89:79-94. https://doi.org/10.1007/s12542-013-0215-z

Larson, D.W. 2008. Diversity and variation of theropod dinosaur teeth from the uppermost Santonian Milk River Formation (Upper Cretaceous), Alberta: a quantitative method supporting identification of the oldest dinosaur tooth assemblage in Canada. Canadian Journal of Earth Sciences, 45:1455-1468. https://doi.org/10.1139/e08-070

Larson, D.W., Brown, C.M., and Evans, D.C. 2016. Dental disparity and ecological stability in bird-like dinosaurs prior to the end-Cretaceous mass extinction. Current Biology, 26:13251333.

Larson, D.W. and Currie, P.J. 2013. Multivariate analyses of small theropod dinosaur teeth and implications for paleoecological turnover through time. PLOS ONE, 8:e54329. https://doi.org/ 10.1371/journal.pone.0054329

Larson, P. 2013. The case for Nanotyrannus, p. 15-53. In Parrish, J.M., Molnar, R.E., Currie, P.J., and Koppelhus, E.B. (eds.), Tyrannosaurid Paleobiology. Indiana University Press, Bloomington, Indiana.

Larsson, H.C.E., Hone, D.W., Dececchi, T.A., Sullivan, C., and Xu, X. 2010. The winged nonavian dinosaur Microraptor fed on mammals: Implications for the Jehol Biota ecosystem. Journal of Vertebrate Paleontology, 30:114A.

Lautenschlager, S. 2013. Cranial myology and bite force performance of Erlikosaurus andrewsi: a novel approach for digital muscle reconstructions. Journal of Anatomy, 222:260-272. https:/ /doi.org/10.1111/joa.12000

Lautenschlager, S. 2014. Morphological and functional diversity in therizinosaur claws and the implications for theropod claw evolution. Proceedings of the Royal Society of London B: Biological Sciences, 281:20140497. https://doi.org/10.1098/rspb.2014.0497

Lautenschlager, S., Witmer, L.M., Perle, A., and Rayfield, E.J. 2013. Edentulism, beaks, and biomechanical innovations in the evolution of theropod dinosaurs. Proceedings of the National Academy of Sciences, 110:20657-20662. https://doi.org/10.1073/pnas.1310711110

Lautenschlager, S., Witmer, L.M., Perle, A., Zanno, L.E., and Rayfield, E.J. 2014. Cranial anatomy of Erlikosaurus andrewsi (Dinosauria, Therizinosauria): new insights based on digital reconstruction. Journal of Vertebrate Paleontology, 34:1263-1291. https://doi.org/ 10.1080/02724634.2014.874529

Lee, M.S.Y., Cau, A., Naish, D., and Dyke, G.J. 2014a. Sustained miniaturization and anatomical innovation in the dinosaurian ancestors of birds. Science, 345:562-566. https://doi.org/ 10.1126/science. 1252243 
Lee, Y.-N., Barsbold, R., Currie, P.J., Kobayashi, Y., Lee, H.-J., Godefroit, P., Escuillié, F., and Chinzorig, T. 2014b. Resolving the long-standing enigmas of a giant ornithomimosaur Deinocheirus mirificus. Nature, 515:257-260. https://doi.org/10.1038/nature13874

Lefèvre, U., Cau, A., Cincotta, A., Hu, D., Chinsamy, A., Escuillié, F., and Godefroit, P. 2017. A new Jurassic theropod from China documents a transitional step in the macrostructure of feathers. The Science of Nature, 104:74. https://doi.org/10.1007/s00114-017-1496-y

Lehman, T.M. and Carpenter, K. 1990. A partial skeleton of the tyrannosaurid dinosaur Aublysodon from the Upper Cretaceous of New Mexico. Journal of Paleontology, 64:10261032.

Leidy, J. 1856. Notice of remains of extinct reptiles and fishes, discovered by Dr. F.V. Hayden in the Bad Lands of the Judith River, Nebraska Territory. Proceedings of the Academy of Natural Sciences of Philadelphia, 8:72-73.

Li, D., Norell, M.A., Gao, K.Q., Smith, N.D., and Makovicky, P.J. 2010. A longirostrine tyrannosauroid from the Early Cretaceous of China. Proceedings of the Royal Society B: Biological Sciences, 277:183-190.

Li, Z., Zhou, Z., and Clarke, J.A. 2018. Convergent evolution of a mobile bony tongue in flighted dinosaurs and pterosaurs. PLoS ONE, 13:e0198078. https://doi.org/10.1371/ journal.pone.0198078

Liao, C.C. and Xu, X. 2019. Cranial osteology of Beipiaosaurus inexpectus (Theropoda: Therizinosauria). Vertebrata PalAsiatica, 57:117-132.

Liyong, J., Jun, C., and Godefroit, P. 2012. A new basal ornithomimosaur (Dinosauria: Theropoda) from the Early Cretaceous Yixian Formation, Northeast China, p. 466-487. In Godefroit, P. (ed.), Bernissart Dinosaurs and Early Cretaceous Terrestrial Ecosystems. Indiana University Press, Bloomington, Indiana.

Long, J.A. and Schouten, P. 2008. Feathered Dinosaurs: the Origin of Birds. Oxford University Press, Oxford, New York.

Longrich, N. 2008. Small theropod teeth from the Lance Formation of Wyoming, USA, p. 135158. In Sankey, J.T. and Baszio, S. (eds.), Vertebrate Microfossil Assemblages: Their Role in Paleoecology and Paleobiogeography. Indiana University Press, Bloomington, Indiana.

Longrich, N.R., Barnes, K., Clark, S., and Millar, L. 2013. Caenagnathidae from the Upper Campanian Aguja Formation of West Texas, and a revision of the Caenagnathinae. Bulletin of the Peabody Museum of Natural History, 54:23-49. https://doi.org/10.3374/014.054.0102

Longrich, N.R. and Currie, P.J. 2009. Albertonykus borealis, a new alvarezsaur (Dinosauria: Theropoda) from the Early Maastrichtian of Alberta, Canada: implications for the systematics and ecology of the Alvarezsauridae. Cretaceous Research, 30:239-252. https://doi.org/ 10.1016/j.cretres.2008.07.005

Longrich, N.R., Pereda-Suberbiola, X., Jalil, N.-E., Khaldoune, F., and Jourani, E. 2017. An abelisaurid from the latest Cretaceous (late Maastrichtian) of Morocco, North Africa. Cretaceous Research, 76:40-52. https://doi.org/10.1016/j.cretres.2017.03.021

Loewen, M.A., Irmis, R.B., Sertich, J.J.W., Currie, P.J., and Sampson, S.D. 2013. Tyrant dinosaur evolution tracks the rise and fall of Late Cretaceous oceans. PLoS ONE, 8:e79420. https://doi.org/10.1371/journal.pone.0079420

Louchart, A. and Viriot, L. 2011. From snout to beak: the loss of teeth in birds. Trends in Ecology \& Evolution, 26:663-673. https://doi.org/10.1016/j.tree.2011.09.004

Lü, J. and Brusatte, S.L. 2015. A large, short-armed, winged dromaeosaurid (Dinosauria: Theropoda) from the Early Cretaceous of China and its implications for feather evolution. Scientific Reports, 5:11775. https://doi.org/10.1038/srep11775

Lü, J., Xu, L., Liu, Y., Zhang, X., Jia, S., and Ji, Q. 2010. A new troodontid theropod from the Late Cretaceous of central China, and the radiation of Asian troodontids. Acta Palaeontologica Polonica, 55:381-388.

Lü, J., Yi, L., Brusatte, S.L., Yang, L., Li, H., and Chen, L. 2014. A new clade of Asian Late Cretaceous long-snouted tyrannosaurids. Nature Communications, 5. https://doi.org/ 10.1038/ncomms4788

Macaluso, L. and Tschopp, E. 2018. Evolutionary changes in pubic orientation in dinosaurs are more strongly correlated with the ventilation system than with herbivory. Palaeontology, 61:703-719. https://doi.org/10.1111/pala.12362

Maddison, W.P. and Maddison, D.R. 2017. Mesquite: a modular system for evolutionary analysis. Version 3.2. Downloaded 16 June 2017 from http://mesquiteproject.org 
Madsen, J.H. 1976. A second new theropod dinosaur from the Late Jurassic of east central Utah. Utah Geology, 3:51-60.

Madsen, J.H. and Welles, S.P. 2000. Ceratosaurus (Dinosauria, Theropoda): a revised osteology. Utah Geological Survey, Miscellaneous Publication, 00-2:1-89.

Madzia, D. 2014. The first non-avian theropod from the Czech Republic. Acta Palaeontologica Polonica, 59:855-862.

Maganuco, S., Cau, A., and Pasini, G. 2005. First description of theropod remains from the Middle Jurassic (Bathonian) of Madagascar. Atti della Società Italiana di Scienze Naturali e del Museo Civico di Storia Naturale di Milano, 146:165-202.

Maisch, M.W. and Matzke, A.T. 2003. Theropods (Dinosauria, Saurischia) from the middle Jurassic Toutunhe Formation of the Southern Junggar Basin, NW China. Paläontologische Zeitschrift, 77:281-292. https://doi.org/10.1007/BF03006942

Makovicky, P.J., Kobayashi, Y., and Currie, P.J. 2004. Ornithomimosauria, p. 137-150. In Weishampel, D.B., Dodson, P., and Osmólska, H. (eds.), The Dinosauria. Second Edition. University of California Press, Berkeley, California.

Makovicky, P.J., Li, D., Gao, K.-Q., Lewin, M., Erickson, G.M., and Norell, M.A. 2010. A giant ornithomimosaur from the Early Cretaceous of China. Proceedings of the Royal Society B: Biological Sciences, 277:191-198. https://doi.org/10.1098/rspb.2009.0236

Makovicky, P.J., Norell, M.A., Clark, J.M., and Rowe, T. 2003. Osteology and relationships of Byronosaurus jaffei (Theropoda: Troodontidae). American Museum Novitates, 3402:1-32. https://doi.org/10.1206/0003-0082(2003)402\%3C0001:oarobj\%3E2.0.co;2

Makovicky, P.J. and Sues, H.D. 1998. Anatomy and phylogenetic relationships of the theropod dinosaur Microvenator celer from the Lower Cretaceous of Montana. American Museum Novitates, 3240:1-27.

Malafaia, E., Escaso, F., Mocho, P., Serrano-Martínez, A., Torices, A., Cachão, M., and Ortega, F. 2017a. Analysis of diversity, stratigraphic and geographical distribution of isolated theropod teeth from the Upper Jurassic of the Lusitanian Basin, Portugal. Journal of Iberian Geology, 43:257-291. https://doi.org/10.1007/s41513-017-0021-7

Malafaia, E., Mocho, P., Escaso, F., and Ortega, F. 2017b. New data on the anatomy of Torvosaurus and other remains of megalosauroid (Dinosauria, Theropoda) from the Upper Jurassic of Portugal. Journal of Iberian Geology, 43:33-59. https://doi.org/10.1007/s41513017-0003-9

Martill, D.M., Vidovic, S.U., Howells, C., and Nudds, J.R. 2016. The oldest Jurassic dinosaur: a basal neotheropod from the Hettangian of Great Britain. PLoS ONE, 11:e0145713. https:// doi.org/10.1371/journal.pone.0145713

Martin, L.D., Stewart, J.D., and Whetstone, K.N. 1980. The origin of birds: structure of the tarsus and teeth. The Auk, 97:86-93.

Martínez, R.N. and Apaldetti, C. 2017. A late Norian-Rhaetian coelophysid neotheropod (Dinosauria, Saurischia) from the Quebrada Del Barro Formation, Northwestern Argentina. Ameghiniana, 54:488-505.

Martínez, R.N., Sereno, P.C., Alcober, O.A., Colombi, C.E., Renne, P.R., Montañez, I.P., and Currie, B.S. 2011. A basal dinosaur from the dawn of the dinosaur era in southwestern Pangaea. Science, 331:206-210. https://doi.org/10.1126/science.1198467.

Martyniuk, M.P. 2012. A Field Guide to Mesozoic Birds and Other Winged Dinosaurs. Pan Aves, Vernon, New Jersey.

Massare, J.A. 1987. Tooth morphology and prey preference of Mesozoic marine reptiles. Journal of Vertebrate Paleontology, 7:121-137. https://doi.org/10.1080/02724634.1987.10011647

Mateus, O., Araújo, R., Natário, C., and Castanhinha, R. 2011. A new specimen of the theropod dinosaur Baryonyx from the Early Cretaceous of Portugal and taxonomic validity of Suchosaurus. Zootaxa, 2827:54-68. https://doi.org/10.11646/zootaxa.2827.1.3

Maxwell, E.E., Caldwell, M.W., Lamoureux, D.O., and Budney, L.A. 2011. Histology of tooth attachment tissues and plicidentine in Varanus (Reptilia: Squamata), and a discussion of the evolution of amniote tooth attachment. Journal of Morphology, 272:1170-1181. https://doi.org/ 10.1002/jmor.10972

Mayr, G., Pohl, B., Hartman, S., and Peters, D.S. 2007. The tenth skeletal specimen of Archaeopteryx. Zoological Journal of the Linnean Society, 149:97-116. https://doi.org/ 10.1111/j.1096-3642.2006.00245.x

Medeiros, M.A. 2006. Large theropod teeth from the Eocenomanian of northeastern Brazil and the occurrence of Spinosauridae. Revista Brasileira de Paleontologia, 9:333-338. 
Melstrom, K.M. 2017. The relationship between diet and tooth complexity in living dentigerous saurians. Journal of Morphology, 278:500-522. https://doi.org/10.1002/jmor.20645

Meredith, R.W., Zhang, G., Gilbert, M.T.P., Jarvis, E.D., and Springer, M.S. 2014. Evidence for a single loss of mineralized teeth in the common avian ancestor. Science, 346:1254390. https:/ /doi.org/10.1126/science.1254390

Milner, A.C. 2002. Theropod dinosaurs of the Purbeck limestone group, Southern England. Special Papers in Palaeontology, 68:191-202.

Mo, J.-Y. and Xu, X. 2015. Large theropod teeth from the Upper Cretaceous of Jiangxi, southern China. Vertebrata PalAsiatica, 53:63-72.

Molnar, R.E. 1978. A new theropod dinosaur from the Upper Cretaceous of central Montana. Journal of Paleontology, 52:73-82.

Molnar, R.E., Obata, I., Tanimoto, M., and Matsukawa, M. 2009. A tooth of Fukuiraptor aff. F. kitadaniensis from the Lower Cretaceous Sebayashi Formation, Sanchu Cretaceous, Japan. Bulletin of Tokyo Gakugei University, Division of Natural Sciences, 61:105-117.

Monfroy, Q.T. 2017. Correlation between the size, shape and position of the teeth on the jaws and the bite force in Theropoda. Historical Biology, 29:1089-1105. https://doi.org/10.1080/ 08912963.2017.1286652

Montgomery, J.M., Gillespie, D., Sastrawan, P., Fredeking, T.M., and Stewart, G.L. 2002. Aerobic salivary bacteria in wild and captive komodo dragons. Journal of Wildlife Diseases, 38:545551. https://doi.org/10.7589/0090-3558-38.3.545

Müller, R.T., Langer, M.C., Bronzati, M., Pacheco, C.P., Cabreira, S.F., and Dias-Da-Silva, S. 2018. Early evolution of sauropodomorphs: anatomy and phylogenetic relationships of a remarkably well-preserved dinosaur from the Upper Triassic of southern Brazil. Zoological Journal of the Linnean Society, 184:1187-1248. https://doi.org/10.1093/zoolinnean/zly009

Naish, D. 2012. Birds, p. 379-423. In Brett-Surman, M.K., Holtz, T.R.J., and Farlow, J.O. (eds.), The Complete Dinosaur, Second Edition. Indiana University Press, Bloomington, Indiana.

Nesbitt, S.J., Denton, R.K., Loewen, M.A., Brusatte, S.L., Smith, N.D., Turner, A.H., Kirkland, J.I., McDonald, A.T., and Wolfe, D.G. 2019. A mid-Cretaceous tyrannosauroid and the origin of North American end-Cretaceous dinosaur assemblages. Nature Ecology \& Evolution, 3:892-899. https://doi.org/10.1038/s41559-019-0888-0

Nesbitt, S.J. and Ezcurra, M.D. 2015. The early fossil record of dinosaurs in North America: A new neotheropod from the base of the Upper Triassic Dockum Group of Texas. Acta Palaeontologica Polonica, 60:513-527. https://doi.org/10.4202/app.00143.2014

Nixon, K.C. 2002. WinClada, version 1.00.08. Published by the author, Ithaca, New York.

Norell, M.A., Clark, J.M., and Chiappe, L.M. 2001a. An embryonic oviraptorid (Dinosauria: Theropoda) from the Upper Cretaceous of Mongolia. American Museum Novitates, 3315:120. https://doi.org/10.1206/0003-0082(2001)315\%3C0001:aeodtf\%3E2.0.co;2

Norell, M.A., Clark, J.M., and Makovicky, P.J. 2001b. Phylogenetic relationships among coelurosaurian theropods, p. 49-67. In Gauthier, J. and Gall, L.F. (eds.), New Perspectives on the Origin and Early Evolution of Birds: Proceedings of the International Symposium in Honor of John H. Ostrom. Peabody Museum of Natural History, Yale University, New Haven.

Norell, M.A., Clark, J.M., Turner, A.H., Makovicky, P.J., Barsbold, R., and Rowe, T. 2006. A new dromaeosaurid theropod from Ukhaa Tolgod (Ömnögov, Mongolia). American Museum Novitates, 3545:1-51. https://doi.org/10.1206/0003-0082(2006)3545[1:andtfu]2.0.co;2

Norell, M.A. and Makovicky, P.J. 2004. Dromaeosauridae, p. 196-209. In Weishampel, D., Dodson, P., and Osmólska, H. (eds.), The Dinosauria. Second Edition. University of California Press, Berkeley, California.

Norell, M.A., Makovicky, P.J., Bever, G.S., Balanoff, A.M., Clark, J.M., Barsbold, R., and Rowe, T. 2009. A review of the Mongolian Cretaceous dinosaur Saurornithoides (Troodontidae: Theropoda). American Museum Novitates, 3654:1-63. https://doi.org/10.1206/648.1

Norell, M.A., Makovicky, P.J., and Clark, J.M. 2000. A new troodontid theropod from Ukhaa Tolgod, Mongolia. Journal of Vertebrate Paleontology, 20:7-11. https://doi.org/10.1671/02724634(2000)020[0007:anttfu]2.0.co;2

Novas, F.E. 1992. La evolución de los dinosaurios carnivoros, p. 126-163. In Sanz, J.L. and Buscalioni, A.D. (eds.), Los Dinosaurios y Su Entorno Biotico: Actas del Segundo Curso de Paleontología en Cuenca. Instituto 'Juan Valdez,' Cuenca, Spain.

Novas, F.E., Agnolín, F.L., Ezcurra, M.D., Porfiri, J., and Canale, J.I. 2013. Evolution of the carnivorous dinosaurs during the Cretaceous: The evidence from Patagonia. Cretaceous Research, 45:174-215. https://doi.org/10.1016/j.cretres.2013.04.001 
Novas, F.E., Chatterjee, S., Rudra, D.K., and Datta, P.M. 2010. Rahiolisaurus gujaratensis, n. gen. n. sp., a new abelisaurid theropod from the Late Cretaceous of India, p. 45-62. In Bandyopadhyay, S. (ed.), New Aspects of Mesozoic Biodiversity. Lecture Notes in Earth Sciences. Springer Berlin Heidelberg.

Novas, F.E., Ezcurra, M.D., Agnolín, F.L., Pol, D., and Ortíz, R. 2012. New Patagonian Cretaceous theropod sheds light about the early radiation of Coelurosauria. Revista del Museo Argentino de Ciencias Naturales, 14:57-81.

Novas, F.E., Ezcurra, M.D., and Lecuona, A. 2008. Orkoraptor burkei nov. gen. et sp., a large theropod from the Maastrichtian Pari Aike Formation, Southern Patagonia, Argentina. Cretaceous Research, 29:468-480. https://doi.org/10.1016/j.cretres.2008.01.001

Novas, F.E., Pol, D., Canale, J.I., Porfiri, J.D., and Calvo, J.O. 2009. A bizarre Cretaceous theropod dinosaur from Patagonia and the evolution of Gondwanan dromaeosaurids. Proceedings of the Royal Society B: Biological Sciences, 276:1101-1107. https://doi.org/ 10.1098/rspb.2008.1554

Novas, F.E., Salgado, L., Suárez, M., Agnolín, F.L., Ezcurra, M.D., Chimento, N.R., de la Cruz, R., Isasi, M.P., Vargas, A.O., and Rubilar-Rogers, D. 2015. An enigmatic plant-eating theropod from the Late Jurassic period of Chile. Nature, 522:331-334. https://doi.org/ 10.1038/nature14307

Novas, F.E., Valais, S., Vickers-Rich, P., and Rich, T. 2005. A large Cretaceous theropod from Patagonia, Argentina, and the evolution of carcharodontosaurids. Naturwissenschaften, 92:226-230. https://doi.org/10.1007/s00114-005-0623-3

O'Connor, J., Zhou, Z., and Xu, X. 2011. Additional specimen of Microraptor provides unique evidence of dinosaurs preying on birds. Proceedings of the National Academy of Sciences, 108:19662-19665. https://doi.org/10.1073/pnas.1117727108

O'Keefe, F.R., Otero, R.A., Soto-Acuña, S., O'gorman, J.P., Godfrey, S.J., and Chatterjee, S. 2017. Cranial anatomy of Morturneria seymourensis from Antarctica, and the evolution of filter feeding in plesiosaurs of the Austral Late Cretaceous. Journal of Vertebrate Paleontology, 37:e1347570. https://doi.org/10.1080/02724634.2017.1347570

Ösi, A., Apesteguía, S., and Kowalewski, M. 2010. Non-avian theropod dinosaurs from the early Late Cretaceous of central Europe. Cretaceous Research, 31:304-320. https://doi.org/ 10.1016/j.cretres.2010.01.001

Osmólska, H., Currie, P.J., and Barsbold, R. 2004. Oviraptorosauria, p. 165-183. In Weishampel, D.B., Dodson, P., and Osmólska, H. (eds.), The Dinosauria. Second Edition. University of California Press, Berkeley, California.

Ostrom, J.H. 1969. Osteology of Deinonychus antirrhopus, an unusual theropod from the Lower Cretaceous of Montana. Bulletin Peabody Museum of Natural History: Peabody Museum of Natural History, Yale University, 30:1-165.

Ostrom, J.H. 1978. The osteology of Compsognathus longipes Wagner. Zitteliana, 4:73-118.

Padian, K. and Chiappe, L.M. 1998. The origin and early evolution of birds. Biological Reviews, 73:1-42. https://doi.org/10.1111/j.1469-185X.1997.tb00024.x

Paul, G.S. 1984. The segnosaurian dinosaurs: relics of the prosauropod-ornithischian transition? Journal of Vertebrate Paleontology, 4:507-515. https://doi.org/10.1080/ 02724634.1984.10012026

Paul, G.S. 2016. The Princeton Field Guide to Dinosaurs. Princeton University Press, Princeton.

Pei, R., Li, Q., Meng, Q., Gao, K.-Q., and Norell, M.A. 2014. A new specimen of Microraptor (Theropoda: Dromaeosauridae) from the Lower Cretaceous of western Liaoning, China. American Museum Novitates, 3821:1-28. https://doi.org/10.1206/3821.1

Pei, R., Li, Q., Meng, Q., Norell, M., and Gao, K. 2017a. New specimens of Anchiornis huxleyi (Theropoda, Paraves) from the late Jurassic of northeastern China. Bulletin of the American Museum of Natural History, 411:1-66. https://doi.org/10.1206/0003-0090-411.1.1

Pei, R., Norell, M.A., Barta, D.E., Bever, G.S., Pittman, M., and Xu, X. 2017b. Osteology of a new Late Cretaceous troodontid specimen from Ukhaa Tolgod, Ömnögovi Aimag, Mongolia. American Museum Novitates, 3889:1-47. https://doi.org/10.1206/3889.1

Pérez-Moreno, B.P., Sanz, J.L., Buscalioni, A.D., Moratalla, J.J., Ortega, F., and RasskinGutman, D. 1994. A unique multitoothed ornithomimosaur dinosaur from the Lower Cretaceous of Spain. Nature, 370:363-367. https://doi.org/10.1038/370363a0

Perle, A., Chiappe, L.M., and Barsbold, R. 1994. Skeletal morphology of Mononykus olecranus (Theropoda: Avialae) from the Late Cretaceous of Mongolia. American Museum Novitates, 3105:1-29. 
Perle, A., Norell, M., and Clark, J.M. 1999. A new maniraptoran theropod, Achillobator giganticus (Dromaeosauridae), from the Upper Cretaceous of Burkhant, Mongolia. Contributions from the Geology and Mineralogy Chair, National Museum of Mongolia, 101:1-105.

Perle, A., Norell, M.A., Chiappe, L.M., and Clark, J.M. 1993. Flightless bird from the Cretaceous of Mongolia. Nature, 362:623-626. https://doi.org/10.1038/362623a0

Peyer, B. 1968. Comparative Odontology. University of Chicago Press. Chicago.

Peyer, K. 2006. A reconsideration of Compsognathus from the upper Tithonian of Canjuers, southeastern France. Journal of Vertebrate Paleontology, 26:879-896. https://doi.org/ 10.1671/0272-4634(2006)26[879:arocft]2.0.co;2

Piechowski, R., Niedźwiedzki, G., and Tałanda, M. 2019. Unexpected bird-like features and high intraspecific variation in the braincase of the Triassic relative of dinosaurs. Historical Biology, 31:1065-1081. https://doi.org/10.1080/08912963.2017.1418339

Pol, D. and Rauhut, O.W.M. 2012. A Middle Jurassic abelisaurid from Patagonia and the early diversification of theropod dinosaurs. Proceedings of the Royal Society B: Biological Sciences, 279:3170-3175. https://doi.org/10.1098/rspb.2012.0660

Porfiri, J.D., Novas, F.E., Calvo, J.O., Agnolín, F.L., Ezcurra, M.D., and Cerda, I.A. 2014. Juvenile specimen of Megaraptor (Dinosauria, Theropoda) sheds light about tyrannosauroid radiation. Cretaceous Research, 51:35-55. https://doi.org/10.1016/j.cretres.2014.04.007

Pu, H., Kobayashi, Y., Lü, J., Xu, L., Wu, Y., Chang, H., Zhang, J., and Jia, S. 2013. An unusual basal therizinosaur dinosaur with an ornithischian dental arrangement from Northeastern China. PLoS ONE, 8:e63423. https://doi.org/10.1371/journal.pone.0063423

Qiu, R., Wang, X., Wang, Q., Li, N., Zhang, J., and Ma, Y. 2019. A new caudipterid from the Lower Cretaceous of China with information on the evolution of the manus of Oviraptorosauria. Scientific Reports, 9:6431. https://doi.org/10.1038/s41598-019-42547-6

Raath, M.A. 1977. The Anatomy of the Triassic Theropod Syntarsus rhodesiensis (Saurischia: Podokesauridae) and a Consideration of its Biology. Ph.D. Dissertation, Department of Zoology and Entomology, Rhodes University, Salisbury, Rhodesia.

Ramírez, M. 2013. Macro CharStats.run v.6 downloaded 23 January 2018. Sistemática Teórica. https://sites.google.com/site/teosiste/tp/archivos.

Rauhut, O.W. 2011. Theropod dinosaurs from the Late Jurassic of Tendaguru (Tanzania). Special Papers in Palaeontology, 86:195-239.

Rauhut, O.W., Hübner, T., and Lanser, K.-P. 2016. A new megalosaurid theropod dinosaur from the late Middle Jurassic (Callovian) of north-western Germany: Implications for theropod evolution and faunal turnover in the Jurassic. Palaeontologia Electronica, 19.2.29A:1-65. https://doi.org/10.26879/654

Rauhut, O.W.M. 2002. Dinosaur teeth from the Barremian of Uña, Province of Cuenca, Spain. Cretaceous Research, 23:255-263. https://doi.org/10.1006/cres.2002.1003

Rauhut, O.W.M. 2003. The interrelationships and evolution of basal theropod dinosaurs. Special Papers in Palaeontology, 69:1-213.

Rauhut, O.W.M. 2004. Provenance and anatomy of Genyodectes serus, a large-toothed ceratosaur (Dinosauria: Theropoda) from Patagonia. Journal of Vertebrate Paleontology, 24:894-902. https://doi.org/10.1671/0272-4634(2004)024[0894:paaogs]2.0.co;2

Rauhut, O.W.M. 2005. Osteology and relationships of a new theropod dinosaur from the Middle Jurassic of Patagonia. Palaeontology, 48:87-110. https://doi.org/10.1111/j.14754983.2004.00436.x

Rauhut, O.W.M. 2014. New observations on the skull of Archaeopteryx. Paläontologische Zeitschrift, 88:211-221. https://doi.org/10.1007/s12542-013-0186-0

Rauhut, O.W.M. and Carrano, M.T. 2016. The theropod dinosaur Elaphrosaurus bambergi Janensch, 1920, from the Late Jurassic of Tendaguru, Tanzania. Zoological Journal of the Linnean Society, 178:546-610. https://doi.org/10.1111/zoj.12425

Rauhut, O.W.M., Foth, C., and Tischlinger, H. 2018. The oldest Archaeopteryx (Theropoda: Avialiae): a new specimen from the Kimmeridgian/Tithonian boundary of Schamhaupten, Bavaria. PeerJ, 6:e4191. https://doi.org/10.7717/peerj.4191

Rauhut, O.W.M., Foth, C., Tischlinger, H., and Norell, M.A. 2012. Exceptionally preserved juvenile megalosauroid theropod dinosaur with filamentous integument from the Late Jurassic of Germany. Proceedings of the National Academy of Sciences, 109:11746-11751. https://doi.org/10.1073/pnas.1203238109

Rauhut, O.W.M. and Kriwet, J. 1994. Teeth of a big theropod dinosaur from Porto das Barcas (Portugal). Berliner Geowissenschaftliche Abhandlungen, E, 13:179-185. 
Rauhut, O.W.M., Milner, A.C., and Moore-Fay, S. 2010. Cranial osteology and phylogenetic position of the theropod dinosaur Proceratosaurus bradleyi (Woodward, 1910) from the Middle Jurassic of England. Zoological Journal of the Linnean Society, 158:155-195. https:// doi.org/10.1111/j.1096-3642.2009.00591.x

Rauhut, O.W.M. and Werner, C. 1995. First record of the family Dromaeosauridae (Dinosauria: Theropoda) in the Cretaceous of Gondwana (Wadi Milk Formation, northern Sudan). Paläontologische Zeitschrift, 69:475-489. https://doi.org/10.1007/BF02987808

Rayfield, E.J., Milner, A.C., Xuan, V.B., and Young, P.G. 2007. Functional morphology of spinosaur 'crocodile-mimic' dinosaurs. Journal of Vertebrate Paleontology, 27:892-901. https://doi.org/10.1671/0272-4634(2007)27[892:fmoscd]2.0.co;2

Reichel, M. 2010. The heterodonty of Albertosaurus sarcophagus and Tyrannosaurus rex: biomechanical implications inferred through 3-D models. Canadian Journal of Earth Sciences, 47:1253-1261. https://doi.org/10.1139/e10-063

Reichel, M. 2012. The variation of angles between anterior and posterior carinae of tyrannosaurid teeth. Canadian Journal of Earth Sciences, 49:477-491. https://doi.org/ 10.1139/e11-068

Reig, O.A. 1963. La presencia de dinosaurios saurisquios en los "Estratos de Ischigualasto" (Mesotriásico Superior) de las provincias de San Juan y La Rioja (República Argentina). Ameghiniana, 3:3-20.

Reisz, R.R. and Sues, H.-D. 2000. Herbivory in late Paleozoic and Triassic terrestrial vertebrates, p. 9-41. In Sues, H.-D. (ed.), Evolution of Herbivory in Terrestrial Vertebrates. Cambridge University Press, New York.

Richter, U., Mudroch, A., and Buckley, L.G. 2013. Isolated theropod teeth from the Kem Kem Beds (Early Cenomanian) near Taouz, Morocco. Paläontologische Zeitschrift, 87:291-309. https://doi.org/10.1007/s12542-012-0153-1

Rodrigues, T. and Kellner, A.W.A. 2013. Taxonomic review of the Ornithocheirus complex (Pterosauria) from the Cretaceous of England. ZooKeys, 308:1-112. https://doi.org/10.3897/ zookeys.308.5559

Rogers, R.R., Eberth, D.A., and Fiorillo, A.R. 2007. Bonebeds: Genesis, Analysis, and Paleobiological Significance. University of Chicago Press, Chicago.

Rowe, T. 1989. A new species of the theropod dinosaur Syntarsus from the Early Jurassic Kayenta Formation of Arizona. Journal of Vertebrate Paleontology, 9:125-136. https://doi.org/ 10.1080/02724634.1989.10011748

Rowe, T. and Gauthier, J. 1990. Ceratosauria, p. 151-168. In Weishampel, D., Dodson, P., and Osmólska, H. (eds.), The Dinosauria. First Edition. University of California Press, Berkeley, California.

Ruiz-Omeñaca, J.I., Piñuela, L., and García-Ramos, J.C. 2012. Primera descripción de dientes de dinosaurios terópodos en la Formación Tereñes (Kimmeridgiense), Asturias. Geogaceta, 52:177-180.

Russell, D.A. and Dong, Z.M. 1993. The affinities of a new theropod from the Alxa Desert, Inner Mongolia, People's Republic of China. Canadian Journal of Earth Sciences, 30:2107-2127. https://doi.org/10.1139/e93-183

Russell, D.A. and Wu, X.-C. 1997. The Crocodylomorpha at and between geological boundaries: the Baden-Powell approach to change? Zoology, 100:164-182.

Russell, L.S. 1948. The dentary of Troödon, a genus of theropod dinosaurs. Journal of Paleontology, 22:625-629.

Ryan, M.J., Currie, P.J., Gardner, J.D., Vickaryous, M.K., and Lavigne, J.M. 1998. Baby hadrosaurid material associated with an unusually high abundance of Troodon teeth from the Horseshoe Canyon Formation, Upper Cretaceous, Alberta, Canada. Gaia, 15:123-133.

Sales, M.A.F. and Schultz, C.L. 2017. Spinosaur taxonomy and evolution of craniodental features: Evidence from Brazil. PLoS ONE, 12:e0187070. https://doi.org/10.1371/ journal.pone. 0187070

Sander, P.M. 1997. Teeth and jaws, p. 717-725. In Currie, P.J. and Padian, K. (eds.), Encyclopedia of Dinosaurs. Academic Press, San Diego, California.

Sander, P.M. 1999. The microstructure of reptilian tooth enamel: terminology, function, and phylogeny. Münchner Geowissenschaftliche Abhandlungen, Reihe A, 38:1-102.

Sander, P.M., Gee, C.T., Hummel, J., and Clauss, M. 2010. Mesozoic plants and dinosaur herbivory, p. 331-359. In Gee, C.T. (ed.), Plants in Mesozoic Time: Morphological Innovations, Phylogeny, Ecosystems. Indiana University Press, Bloomington. 
Sanderson, M.J. and Donoghue, M.J. 1989. Patterns of variation in levels of homoplasy. Evolution, 43:1781-1795. https://doi.org/10.1111/j.1558-5646.1989.tb02626.x

Sankey, J.T. 2001. Late Campanian southern dinosaurs, Aguja Formation, Big Bend, Texas. Journal of Paleontology, 75:208-215. https://doi.org/10.1017/s0022336000031991

Sankey, J.T. 2008. Diversity of Latest Cretaceous (Late Maastrichtian) small theropods and birds: teeth from the Lance and Hell Creek Formations, USA, p. 117-134. In Sankey, J.T. and Baszio, S. (eds.), Vertebrate Microfossil Assemblages: Their Role in Paleoecology and Paleobiogeography. Indiana University Press, Bloomington, Indiana.

Sankey, J.T., Brinkman, D.B., Guenther, M., and Currie, P.J. 2002. Small theropod and bird teeth from the Late Cretaceous (Late Campanian) Judith River Group, Alberta. Journal of Paleontology, 76:751-763. https://doi.org/10.1666/00223360(2002)076\%3C0751:stabtf\%3E2.0.co;2

Schwenk, K. 2000. Feeding: Form, Function and Evolution in Tetrapod Vertebrates. Academic Press, San Diego.

Senter, P. 2007. A new look at the phylogeny of Coelurosauria (Dinosauria: Theropoda). Journal of Systematic Palaeontology, 5:429-463. https://doi.org/10.1017/S1477201907002143

Senter, P. 2011. Using creation science to demonstrate evolution 2: morphological continuity within Dinosauria. Journal of Evolutionary Biology, 24:2197-2216. https://doi.org/10.1111/ j.1420-9101.2011.02349.x

Senter, P., Barsbold, R., Britt, B.B., and Burnham, D.A. 2004. Systematics and evolution of Dromaeosauridae (Dinosauria, Theropoda). Bulletin of Gunma Museum of Natural History, 8:1-20.

Sereno, P.C. 1999. The evolution of dinosaurs. Science, 284:2137-2147. https://doi.org/10.1126/ science.284.5423.2137

Sereno, P.C. 2017. Early Cretaceous Ornithomimosaurs (Dinosauria: Coelurosauria) from Africa. Ameghiniana, 54:576-616. https://doi.org/10.5710/AMGH.23.10.2017.3155

Sereno, P.C., Beck, A.L., Dutheil, D.B., Gado, B., Larsson, H.C.E., Lyon, G.H., Marcot, J.D., Rauhut, O.W.M., Sadleir, R.W., Sidor, C.A., Varricchio, D.D., Wilson, G.P., and Wilson, J.A. 1998. A long-snouted predatory dinosaur from Africa and the evolution of spinosaurids. Science, 282:1298-1302. https://doi.org/10.1126/science.282.5392.1298

Sereno, P.C., Dutheil, D.B., Larochene, M., Larsson, H.C.E., Lyon, G.H., Magwene, P.M., Sidor, C.A., Varricchio, D.J., and Wilson, J.A. 1996. Predatory dinosaurs from the Sahara and Late Cretaceous faunal differentiation. Science, 272:986-991. https://doi.org/10.1126/ science.272.5264.986

Sereno, P.C., Forster, C.A., Rogers, R.R., and Monetta, A.M. 1993. Primitive dinosaur skeleton from Argentina and the early evolution of Dinosauria. Nature, 361:64-66. https://doi.org/ 10.1038/361064a0

Sereno, P.C., Martínez, R.N., and Alcober, O.A. 2013. Osteology of Eoraptor lunensis (Dinosauria, Sauropodomorpha). Journal of Vertebrate Paleontology, 32:83-179. https:// doi.org/10.1080/02724634.2013.820113

Sereno, P.C. and Novas, F.E. 1994. The skull and neck of the basal theropod Herrerasaurus ischigualastensis. Journal of Vertebrate Paleontology, 13:451-476. https://doi.org/10.1080/ 02724634.1994.10011525

Sereno, P.C., Tan, L., Brusatte, S.L., Kriegstein, H.J., Zhao, X., and Cloward, K. 2009. Tyrannosaurid skeletal design first evolved at small body size. Science, 326:418-422. https:// doi.org/10.1126/science.1177428

Sereno, P.C., Wilson, J.A., and Conrad, J.L. 2004. New dinosaurs link southern landmasses in the Mid-Cretaceous. Proceedings of the Royal Society of London. Series B: Biological Sciences, 271:1325-1330. https://doi.org/10.1098/rspb.2004.2692

Serrano-Brañas, C.I., Gutiérrez-Blando, C., Bigurra, R.D., and González-León, C.M. 2017. First occurrence of tyrannosaurid theropods from the Corral de Enmedio Formation (Upper Cretaceous) Sonora, México. Cretaceous Research, 75:81-93. https://doi.org/10.1016/ j.cretres.2017.03.015

Serrano-Martínez, A., Ortega, F., Sciscio, L., Tent-Manclús, J.E., Fierro Bandera, I., and Knoll, F. 2015. New theropod remains from the Tiourarén Formation (?Middle Jurassic, Niger) and their bearing on the dental evolution in basal tetanurans. Proceedings of the Geologists' Association, 126:107-118. https://doi.org/10.1016/j.pgeola.2014.10.005 
Serrano-Martínez, A., Vidal, D., Sciscio, L., Ortega, F., and Knoll, F. 2016. Isolated theropod teeth from the Middle Jurassic of Niger and the early dental evolution of Spinosauridae. Acta Palaeontologica Polonica, 61:403-415. https://doi.org/10.4202/app.00101.2014

Shen, C., Lü, J., Liu, S., Kundrát, M., Brusatte, S.L., and Gao, H. 2017a. A new troodontid dinosaur from the Lower Cretaceous Yixian Formation of Liaoning Province, China. Acta Geologica Sinica - English Edition, 91:763-780. https://doi.org/10.1111/1755-6724.13307

Shen, C., Zhao, B., Gao, C., Lü, J., and Kundrát, M. 2017b. A new troodontid dinosaur (Liaoningvenator curriei gen. et sp. nov.) from the Early Cretaceous Yixian Formation in western Liaoning province. Acta Geoscientia Sinica, 38:359-371.

Smith, J.B. 2005. Heterodonty in Tyrannosaurus rex: implications for the taxonomic and systematic utility of theropod dentitions. Journal of Vertebrate Paleontology, 25:865-887. https://doi.org/10.1671/0272-4634(2005)025[0865:hitrif]2.0.co;2

Smith, J.B. 2007. Dental morphology and variation in Majungasaurus crenatissimus (Theropoda: Abelisauridae) from the Late Cretaceous of Madagascar. Journal of Vertebrate Paleontology, 27:103-126. https://doi.org/10.1671/0272-4634(2007)27[103:dmavim]2.0.co;2

Smith, J.B. and Dalla Vecchia, F.M. 2006. An abelisaurid (Dinosauria: Theropoda) tooth from the Lower Cretaceous Chicla formation of Libya. Journal of African Earth Sciences, 46:240-244. https://doi.org/10.1016/j.jafrearsci.2006.05.007

Smith, J.B. and Dodson, P. 2003. A proposal for a standard terminology of anatomical notation and orientation in fossil vertebrate dentitions. Journal of Vertebrate Paleontology, 23:1-12. https://doi.org/10.1671/0272-4634(2003)23[1:apfast]2.0.co;2

Smith, J.B. and Lamanna, M.C. 2006. An abelisaurid from the Late Cretaceous of Egypt: implications for theropod biogeography. Naturwissenschaften, 93:242-245. https://doi.org/ 10.1007/s00114-006-0092-3

Smith, J.B., Vann, D.R., and Dodson, P. 2005. Dental morphology and variation in theropod dinosaurs: Implications for the taxonomic identification of isolated teeth. The Anatomical Record Part A: Discoveries in Molecular, Cellular, and Evolutionary Biology, 285A:699-736. https://doi.org/10.1002/ar.a.20206

Smith, N.D., Makovicky, P.J., Agnolín, F.L., Ezcurra, M.D., Pais, D.F., and Salisbury, S.W. 2008. A Megaraptor-like theropod (Dinosauria: Tetanurae) in Australia: support for faunal exchange across eastern and western Gondwana in the Mid-Cretaceous. Proceedings of the Royal Society B: Biological Sciences, 275:2085-2093. https://doi.org/10.1098/rspb.2008.0504

Snively, E., Henderson, D.M., and Phillips, D.S. 2006. Fused and vaulted nasals of tyrannosaurid dinosaurs: implications for cranial strength and feeding mechanics. Acta Palaeontologica Polonica, 51:435-454.

Soto, M. and Perea, D. 2008. A ceratosaurid (Dinosauria, Theropoda) from the Late JurassicEarly Cretaceous of Uruguay. Journal of Vertebrate Paleontology, 28:439-444. https://doi.org/ 10.1671/0272-4634(2008)28[439:acdtft]2.0.co;2

Stromer, E. 1915. Ergebnisse der Forschungsreisen Prof. E. Stromers in den Wüsten Ägyptens. II. Wirbeltier-Reste der Baharîje-Stufe (unterstes Cenoman). 3. Das Original des Theropoden Spinosaurus aegyptiacus nov. gen., nov. spec. Abhandlungen der Königlichen Bayerischen Akademie der Wissenschaften, Mathematisch- Physikalische Klasse, 28:1-32.

Stromer, E. 1931. Wirbeltier-Reste der Baharije-Stufe (unterstes Cenoman). 10. Ein Skelett-Rest von Carcharodontosaurus nov. gen. Abhandlungen der Bayerischen Akademie der Wissenschaften Mathematisch-naturwissenschaftliche Abteilung, 9:1-31.

Stromer, E. 1934. Die Zähne des Compsognathus und Bemerkungen über das Gebiss der Theropoda. Centralblatt für Mineralogie, Geologie und Paläontologie, Abteilung B, 4:74-85.

Sues, H.-D. 1977. The skull of Velociraptor mongoliensis, a small cretaceous theropod dinosaur from Mongolia. Paläontologische Zeitschrift, 51(3-4):173-184.

Sues, H.-D. 2000. Herbivory in terrestrial vertebrates: an introduction, p. 1-8. In Sues, H.-D. (ed.), Evolution of Herbivory in Terrestrial Vertebrates. Cambridge University Press, Cambridge, U.K.; New York.

Sues, H.-D. and Averianov, A. 2013. Enigmatic teeth of small theropod dinosaurs from the Upper Cretaceous (Cenomanian-Turonian) of Uzbekistan. Canadian Journal of Earth Sciences, 50:306-314. https://doi.org/10.1139/e2012-033

Sues, H.-D., Frey, E., Martill, D.M., and Scott, D.M. 2002. Irritator challengeri, a spinosaurid (Dinosauria: Theropoda) from the Lower Cretaceous of Brazil. Journal of Vertebrate Paleontology, 22:535-547. https://doi.org/10.1671/0272-4634(2002)022[0535:icasdt]2.0.co;2 
Sues, H.-D., Nesbitt, S.J., Berman, D.S., and Henrici, A.C. 2011. A late-surviving basal theropod dinosaur from the latest Triassic of North America. Proceedings of the Royal Society B: Biological Sciences, 278:3459-3464. https://doi.org/10.1098/rspb.2011.0410

Suzuki, S., Chiappe, L.M., and Dyke, G.J. 2002. A new specimen of Shuvuuia deserti Chiappe et al., 1998, from the Mongolian Late Cretaceous with a discussion of the relationships of alvarezsaurids to other theropod dinosaurs. Contributions in Science: Natural History Museum of Los Angeles County, 194:1-18.

Sweetman, S.C. 2004. The first record of velociraptorine dinosaurs (Saurischia, Theropoda) from the Wealden (Early Cretaceous, Barremian) of southern England. Cretaceous Research, 25:353-364. https://doi.org/10.1016/j.cretres.2004.01.004

Tanke, D.H. and Currie, P.J. 1998. Head-biting behavior in theropod dinosaurs. Gaia, 15:167184.

Taquet, P. 1984. Une curieuse spécialisation du crâne de certains dinosaures carnivores du Crétacé: le museau long et étroit des spinosauridés. Comptes-rendus des Séances de l'Académie des Sciences. Série 2, Mécanique-physique, Chimie, Sciences de l'Univers, Sciences de la Terre, 299:217-222.

Taquet, P. and Russell, D.A. 1998. New data on spinosaurid dinosaurs from the Early Cretaceous of the Sahara. Comptes Rendus de l'Académie des Sciences-Series IIA-Earth and Planetary Science, 327:347-353. https://doi.org/10.1016/s1251-8050(98)80054-2

Tavares, S.A.S., Ricardi Branco, F., and Santucci, R.M. 2014. Theropod teeth from the Adamantina Formation (Bauru Group, Upper Cretaceous), Monte Alto, São Paulo, Brazil. Cretaceous Research, 50:59-71. https://doi.org/10.1016/j.cretres.2014.03.021

Tehrani, H., Tejero-Trujeque, R., and Dhital, S.K. 2008. Re: Septic arthritis due to a Savannah Monitor lizard bite: a case report. Journal of Hand Surgery (European Volume), 33:810-810. https://doi.org/10.1177/1753193408092495

Therrien, F., Henderson, D.M., and Ruff, C.B. 2005. Bite me: biomechanical models of theropod mandibles and implications for feeding behavior, p. 179-237. In Carpenter, K. (ed.), The Carnivorous Dinosaurs. Indiana University Press, Bloomington, Indiana.

Torices, A., Currie, P.J., Canudo, J.I. and Pereda-Suberbiola, X. 2015. Theropod dinosaurs from the Upper Cretaceous of the South Pyrenees Basin of Spain. Acta Palaeontologica Polonica, 60:611-626.

Torices, A., Wilkinson, R., Arbour, V.M., Ruiz-Omeñaca, J.I., and Currie, P.J. 2018. Punctureand-pull biomechanics in the teeth of predatory coelurosaurian dinosaurs. Current Biology, 28:1467-1474.e2. https://doi.org/10.1016/j.cub.2018.03.042

Tortosa, T., Buffetaut, E., Vialle, N., Dutour, Y., Turini, E., and Cheylan, G. 2014. A new abelisaurid dinosaur from the Late Cretaceous of southern France: Palaeobiogeographical implications. Annales de Paléontologie, 100:63-86. https://doi.org/10.1016/ j.annpal.2013.10.003

Tsuihiji, T., Barsbold, R., Watabe, M., Tsogtbaatar, K., Chinzorig, T., Fujiyama, Y., and Suzuki, S. 2014. An exquisitely preserved troodontid theropod with new information on the palatal structure from the Upper Cretaceous of Mongolia. Naturwissenschaften, 101:131-142. https:/ /doi.org/10.1007/s00114-014-1143-9

Tsuihiji, T., Watabe, M., Tsogtbaatar, K., Tsubamoto, T., Barsbold, R., Suzuki, S., Lee, A.H., Ridgely, R.C., Kawahara, Y., and Witmer, L.M. 2011. Cranial osteology of a juvenile specimen of Tarbosaurus bataar (Theropoda, Tyrannosauridae) from the Nemegt Formation (Upper Cretaceous) of Bugin Tsav, Mongolia. Journal of Vertebrate Paleontology, 31:497517. https://doi.org/10.1080/02724634.2011.557116

Tsuihiji, T., Witmer, L.M., Watabe, M., Barsbold, R., Tsogtbaatar, K., Suzuki, S., and Khatanbaatar, P. 2017. New information on the cranial morphology of Avimimus (Theropoda: Oviraptorosauria). Journal of Vertebrate Paleontology, 37(4):e1347177 https://doi.org/ 10.1080/02724634.2017.1347177

Turner, A.H., Hwang, S.H., and Norell, M.A. 2007. A small derived theropod from Öösh, Early Cretaceous, Baykhangor Mongolia. American Museum Novitates, 3557:1-27. https://doi.org/ 10.1206/0003-0082(2007)3557[1:asdtfs]2.0.co;2

Turner, A.H., Makovicky, P.J., and Norell, M. 2012. A review of dromaeosaurid systematics and paravian phylogeny. Bulletin of the American Museum of Natural History, 371:1-206. https:// doi.org/10.1206/748.1

Turner, A.H., Pol, D., and Norell, M.A. 2011. Anatomy of Mahakala omnogovae (Theropoda: Dromaeosauridae), Tögrögiin Shiree, Mongolia. American Museum Novitates, 3722:1-66. 
Tykoski, R.S. 1998. The Osteology of Syntarsus kayentakatae and Its Implications for Ceratosaurid Phylogeny. MSc Thesis, University of Texas at Austin, Austin, Texas, USA.

Tykoski, R.S. 2005. Anatomy, Ontogeny, and Phylogeny of Coelophysoid Theropods. Ph.D. Dissertation, The University of Texas, Austin, Texas, USA.

Tykoski, R.S. and Rowe, T. 2004. Ceratosauria, p. 47-70. In Weishampel, D., Dodson, P., and Osmólska, H. (eds.), The Dinosauria. Second Edition. University of California Press, Berkeley, California.

Ungar, P.S. 2010. Mammal Teeth: Origin, Evolution, and Diversity. Johns Hopkins University Press, Baltimore.

Upchurch, P. and Barrett, P.M. 2000. The evolution of sauropod feeding mechanisms, p. 79-122. In Sues, H.-D. (ed.), Evolution of Herbivory in Terrestrial Vertebrates: Perspectives from the Fossil Record. Cambridge University Press, Cambridge, U.K.; New York.

van der Lubbe, T., Richter, U. and Knötschke, N. 2009. Velociraptorine dromaeosaurid teeth from the Kimmeridgian (Late Jurassic) of Germany. Acta Palaeontologica Polonica, 54:401-408. https://doi.org/10.4202/app.2008.0007

Varricchio, D.J. 1997. Troodontidae, p. 749-754. In Currie, P.J. and Padian, K. (eds.), Encyclopedia of Dinosaurs. Academic Press, San Diego, California.

Varricchio, D.J., Horner, J.R., and Jackson, F.D. 2002. Embryos and eggs for the Cretaceous theropod dinosaur Troodon formosus. Journal of Vertebrate Paleontology, 22:564-576. https:/ /doi.org/10.1671/0272-4634(2002)022[0564:eaeftc]2.0.co;2

Vikrant, S. and Verma, B.S. 2014. Monitor lizard bite-induced acute kidney injury - a case report. Renal Failure, 36:444-446. https://doi.org/10.3109/0886022X.2013.868223

von Meyer, H. 1861. Archaeopteryx lithographica (Vogel-Feder) und Pterodactylus von Solnhofen. Neues Jahrbuch für Mineralogie, Geognosie, Geologie und Petrefakten-Kunde, 1861:678-679.

Vullo, R., Abit, D., Ballevre, M., Billon-Bruyat, J.-P., Bourgeais, R., Buffetaut, E., Daviero-Gomez, V., Garcia, G., Gomez, B., and Mazin, J.-M. 2014. Palaeontology of the Purbeck-type (Tithonian, Late Jurassic) bonebeds of Chassiron (Oléron Island, western France). Comptes Rendus Palevol, 13:421-441. https://doi.org/10.1016/j.crpv.2014.03.003

Vullo, R. and Néraudeau, D. 2010. Additional dinosaur teeth from the Cenomanian (Late Cretaceous) of Charentes, southwestern France. Comptes Rendus Palevol, 9:121-126. https://doi.org/10.1016/j.crpv.2010.03.001

Vullo, R., Néraudeau, D., and Lenglet, T. 2007. Dinosaur teeth from the Cenomanian of Charentes, Western France: evidence for a mixed Laurasian-Gondwanan assemblages. Journal of Vertebrate Paleontology, 27:931-943. https://doi.org/10.1671/02724634(2007)27[931:dtftco]2.0.co;2

Wang, C.-C., Song, Y.-F., Song, S.-R., Ji, Q., Chiang, C.-C., Meng, Q., Li, H., Hsiao, K., Lu, Y.-C., Shew, B.-Y., Huang, T., and Reisz, R.R. 2015. Evolution and function of dinosaur teeth at ultramicrostructural level revealed using synchrotron transmission $\mathrm{x}$-ray microscopy. Scientific Reports, 5:srep15202. https://doi.org/10.1038/srep15202

Wang, S., Stiegler, J., Amiot, R., Wang, X., Du, G., Clark, J.M., and Xu, X. 2017a. Extreme ontogenetic changes in a ceratosaurian theropod. Current Biology, 27:144-148. https:// doi.org/10.1016/j.cub.2016.10.043

Wang, S., Stiegler, J., Wu, P., Chuong, C.-M., Hu, D., Balanoff, A., Zhou, Y., and Xu, X. 2017b. Heterochronic truncation of odontogenesis in theropod dinosaurs provides insight into the macroevolution of avian beaks. Proceedings of the National Academy of Sciences, 114:10930-10935. https://doi.org/10.1073/pnas.1708023114

Watabe, M., Weishampel, D.B., Barsbold, R., and Tsogtbaatar, K. 2000. New nearly complete skeleton of the bird-like theropod, Avimimus, from the Upper Cretaceous of the Gobi Desert, Mongolia. Journal of Vertebrate Paleontology, 20:77A.

Watanabe, A. and Sereno, P. 2010. A large short-snouted dromaeosaurid (Theropoda: Maniraptora) from Inner Mongolia. 70th Annual Meeting Society of Vertebrate Paleontology, Pittsburgh, Pennsylvania, USA (October 10-13, 2010), Program and Abstracts, p. 184A.

Welles, S.P. 1984. Dilophosaurus wetherilli (Dinosauria, Theropoda). Osteology and comparisons. Palaeontographica Abteilung A, 185:85-180.

White, M.A., Bell, P.R., Cook, A.G., Poropat, S.F., and Elliott, D.A. 2015. The dentary of Australovenator wintonensis (Theropoda, Megaraptoridae); implications for megaraptorid dentition. PeerJ, 3:e1512. https://doi.org/10.7717/peerj.1512 
Wilson, J.A. 2006. Anatomical nomenclature of fossil vertebrates: standardized terms or 'lingua franca'? Journal of Vertebrate Paleontology, 26:511-518. https://doi.org/10.1671/02724634(2006)26[511:anofvs]2.0.co;2

Wings, O. 2007. A review of gastrolith function with implications for fossil vertebrates and a revised classification. Acta Palaeontologica Polonica, 52:1-16.

Wings, O. and Sander, P.M. 2007. No gastric mill in sauropod dinosaurs: new evidence from analysis of gastrolith mass and function in ostriches. Proceedings of the Royal Society of London B: Biological Sciences, 274:635-640. https://doi.org/10.1098/rspb.2006.3763

Wongko, K., Buffetaut, E., Khamka, S., and Lauprasert, K. 2019. Spinosaurid theropod teeth from the Red Beds of the Khok Kruat Formation (Early Cretaceous) in Northeastern Thailand. Tropical Natural History, 19:8-20.

Xing, L. 2012. Sinosaurus from Southwestern China. MSc. Dissertation, University of Alberta, Edmonton, Alberta, Canada.

Xing, L., Bell, P.R., Persons, W.S., Ji, S., Miyashita, T., Burns, M.E., Ji, Q., and Currie, P.J. 2012. Abdominal contents from two large Early Cretaceous compsognathids (Dinosauria: Theropoda) demonstrate feeding on confuciusornithids and dromaeosaurids. PLOS ONE, 7:e44012. https://doi.org/10.1371/journal.pone.0044012

Xing, L., Bell, P.R., Rothschild, B.M., Ran, H., Zhang, J., Dong, Z., Zhang, W., and Currie, P.J. 2013a. Tooth loss and alveolar remodeling in Sinosaurus triassicus (Dinosauria: Theropoda) from the Lower Jurassic strata of the Lufeng Basin, China. Chinese Science Bulletin, 58:1931-1935. https://doi.org/10.1007/s11434-013-5765-7

Xing, L., Persons, W.S., Bell, P.R., Xu, X., Zhang, J., Miyashita, T., Wang, F., and Currie, P.J. 2013b. Piscivory in the feathered dinosaur Microraptor. Evolution, 67:2441-2445. https:// doi.org/10.1111/evo.12119

Xu, X., Cheng, Y.-N., Wang, X.-L., and Chang, C.-H. 2002a. An unusual oviraptorosaurian dinosaur from China. Nature, 419:291-293. https://doi.org/10.1038/nature00966

Xu, X., Choiniere, J., Tan, Q., Benson, R.B.J., Clark, J., Sullivan, C., Zhao, Q., Han, F., Ma, Q., He, Y., Wang, S., Xing, H., and Tan, L. 2018. Two Early Cretaceous fossils document transitional stages in alvarezsaurian dinosaur evolution. Current Biology, 28:1-8. https:// doi.org/10.1016/j.cub.2018.07.057

Xu, X., Choiniere, J.N., Pittman, M.D., Tan, Q., Xiao, D., Li, Z., Tan, L., Clark, J.M., Norell, M.A., and Hone, D.W. 2010. A new dromaeosaurid (Dinosauria: Theropoda) from the Upper Cretaceous Wulansuhai Formation of Inner Mongolia, China. Zootaxa, 2403:1-9. https:// doi.org/10.11646/zootaxa.2403.1.1

$\mathrm{Xu}, \mathrm{X}$. and Clark, J.M. 2008. The presence of a gigantic theropod in the Jurassic Shishugou Formation, Junggar Basin, western China. Vertebrata PalAsiatica, 46:57-160.

Xu, X., Clark, J.M., Forster, C.A., Norell, M.A., Erickson, G.M., Eberth, D.A., Jia, C., and Zhao, Q. 2006. A basal tyrannosauroid dinosaur from the Late Jurassic of China. Nature, 439:715718. https://doi.org/10.1038/nature04511

Xu, X., Clark, J.M., Mo, J., Choiniere, J., Forster, C.A., Erickson, G.M., Hone, D.W.E., Sullivan, C., Eberth, D.A., Nesbitt, S., Zhao, Q., Hernandez, R., Jia, C., Han, F., and Guo, Y. 2009. A Jurassic ceratosaur from China helps clarify avian digital homologies. Nature, 459:940-944. https://doi.org/10.1038/nature08124

Xu, X., Currie, P., Pittman, M., Xing, L., Meng, Q., Lü, J., Hu, D., and Yu, C. 2017. Mosaic evolution in an asymmetrically feathered troodontid dinosaur with transitional features. Nature Communications, 8:ncomms14972. https://doi.org/10.1038/ncomms14972

$\mathrm{Xu}, \mathrm{X}$. and Li, F. 2016. A new microraptorine specimen (Theropoda: Dromaeosauridae) with a brief comment on the evolution of compound bones in theropods. Vertebrata PalAsiatica, 54:269-285.

$\mathrm{Xu}, \mathrm{X}$. and Norell, M.A. 2004. A new troodontid dinosaur from China with avian-like sleeping posture. Nature, 431:838-841. https://doi.org/10.1038/nature02898

Xu, X., Norell, M.A., Kuang, X., Wang, X., Zhao, Q., and Jia, C. 2004. Basal tyrannosauroids from China and evidence for protofeathers in tyrannosauroids. Nature, 431:680-684. https:// doi.org/10.1038/nature02855

Xu, X., Norell, M.A., Wang, X., Makovicky, P.J., and Wu, X. 2002b. A basal troodontid from the Early Cretaceous of China. Nature, 415:780-784. https://doi.org/10.1038/415780a 
Xu, X., Pittman, M.D., Sullivan, C., Choiniere, J.N., Tan, Q.W., Clark, J.M., Norell, M.A., and Wang, S. 2015a. The taxonomic status of the Late Cretaceous dromaeosaurid Linheraptor exquisitus and its implications for dromaeosaurid systematics. Vertebrata PalAsiatica, 53:2962.

Xu, X., Tan, Q., Sullivan, C., Han, F., and Xiao, D. 2011a. A short-armed troodontid dinosaur from the Upper Cretaceous of Inner Mongolia and its implications for troodontid evolution. PLoS ONE, 6:e22916. https://doi.org/10.1371/journal.pone.0022916

Xu, X., Tang, Z., and Wang, X. 1999. A therizinosauroid dinosaur with integumentary structures from China. Nature, 399:350-354. https://doi.org/10.1038/20670

$\mathrm{Xu}, \mathrm{X}$. and Wang, X.-L. 2004. A new troodontid (Theropoda: Troodontidae) from the Lower Cretaceous Yixian Formation of western Liaoning, China. Acta Geologica Sinica, 78:22-26.

$\mathrm{Xu}, \mathrm{X}$. and Wu, X.-C. 2001. Cranial morphology of Sinornithosaurus millenii Xu et al. 1999 (Dinosauria: Theropoda: Dromaeosauridae) from the Yixian Formation of Liaoning, China. Canadian Journal of Earth Sciences, 38:1739-1752. https://doi.org/10.1139/e01-082

Xu, X., Xhou, Z., Sullivan, C., Wang, Y., and Ren, D. 2016. An updated review of the Middle-Late Jurassic Yanliao biota: chronology, taphonomy, paleontology and paleoecology. Acta Geologica Sinica-English Edition, 90:2229-2243. https://doi.org/10.1111/1755-6724.13033

Xu, X., You, H., Du, K., and Han, F. 2011b. An Archaeopteryx-like theropod from China and the origin of Avialae. Nature, 475:465-470. https://doi.org/10.1038/nature10288

Xu, X., Zhao, X., and Clark, J.M. 2001. A new therizinosaur from the Lower Jurassic lower Lufeng Formation of Yunnan, China. Journal of Vertebrate Paleontology, 21:477-483. https:// doi.org/10.1671/0272-4634(2001)021[0477:antftl]2.0.co;2

Xu, X., Zheng, X., Sullivan, C., Wang, X., Xing, L., Wang, Y., Zhang, X., O'Connor, J.K., Zhang, F., and Pan, Y. 2015b. A bizarre Jurassic maniraptoran theropod with preserved evidence of membranous wings. Nature, 521:70-73. https://doi.org/10.1038/nature14423

Xu, X., Zhou, Z., and Wang, X. 2000. The smallest known non-avian theropod dinosaur. Nature, 408:705-708. https://doi.org/10.1038/35047056

Yang, T.-R. and Sander, P.M. 2018. The origin of the bird's beak: new insights from dinosaur incubation periods. Biology Letters, 14:20180090. https://doi.org/10.1098/rsbl.2018.0090

Yates, A.M. 2005. A new theropod dinosaur from the Early Jurassic of South Africa and its implications for the early evolution of theropods. Palaeontologia Africana, 41:105-122.

You, H.-L., Azuma, Y., Wang, T., Wang, Y.-M., and Dong, Z.-M. 2014. The first well-preserved coelophysoid theropod dinosaur from Asia. Zootaxa, 3873:233. https://doi.org/10.11646/ zootaxa.3873.3.3

Young, C.M.E., Hendrickx, C., Challands, T.J., Foffa, D., Ross, D.A., Butler, I.B., and Brusatte, S.L. 2019. New theropod dinosaur teeth from the Middle Jurassic of the Isle of Skye, Scotland. Scottish Journal of Geology, 55:7-19. https://doi.org/10.1144/sjg2018-020

Zanno, L.E. 2010a. Osteology of Falcarius utahensis (Dinosauria: Theropoda): characterizing the anatomy of basal therizinosaurs. Zoological Journal of the Linnean Society, 158:196-230. https://doi.org/10.1111/j.1096-3642.2009.00464.x

Zanno, L.E. 2010b. A taxonomic and phylogenetic re-evaluation of Therizinosauria (Dinosauria: Maniraptora). Journal of Systematic Palaeontology, 8:503-543. https://doi.org/10.1080/ 14772019.2010.488045

Zanno, L.E., Gillette, D.D., Albright, L.B., and Titus, A.L. 2009. A new North American therizinosaurid and the role of herbivory in 'predatory' dinosaur evolution. Proceedings of the Royal Society B: Biological Sciences, 276:3505-3511. https://doi.org/10.1098/ rspb.2009.1029

Zanno, L.E. and Makovicky, P.J. 2011. Herbivorous ecomorphology and specialization patterns in theropod dinosaur evolution. Proceedings of the National Academy of Sciences, 108:232237. https://doi.org/10.1073/pnas.1011924108

Zanno, L.E., Tsogtbaatar, K., Chinzorig, T., and Gates, T.A. 2016. Specializations of the mandibular anatomy and dentition of Segnosaurus galbinensis (Theropoda: Therizinosauria). PeerJ, 4:e1885. https://doi.org/10.7717/peerj.1885

Zanno, L.E., Tucker, R.T., Canoville, A., Avrahami, H.M., Gates, T.A., and Makovicky, P.J. 2019. Diminutive fleet-footed tyrannosauroid narrows the 70-million-year gap in the North American fossil record. Communications Biology, 2:64. https://doi.org/10.1038/s42003-019-0308-7

Zhang, F., Zhou, Z., Xu, X., and Wang, X. 2002. A juvenile coelurosaurian theropod from China indicates arboreal habits. Naturwissenschaften, 89:394-398. https://doi.org/10.1007/s00114002-0353-8 
Zhang, F., Zhou, Z., Xu, X., Wang, X., and Sullivan, C. 2008. A bizarre Jurassic maniraptoran from China with elongate ribbon-like feathers. Nature, 455:1105-1108. https://doi.org/ 10.1038/nature07447

Zhang, X.-H., Xu, X., Zhao, X.-J., Sereno, P., Kuang, X.-W., and Tan, L. 2001. A long-necked therizinosauroid dinosaur from the Upper Cretaceous Iren Dabasu Formation of nei Mongol, People's Republic of China. Vertebrata PalAsiatica, 39:283-294.

Zhao, X. and Xu, X. 1998. The oldest coelurosaurian. Nature, 394:234-235. https://doi.org/ $10.1038 / 28300$

Zheng, X., Xu, X., You, H., Zhao, Q., and Dong, Z. 2010. A short-armed dromaeosaurid from the Jehol Group of China with implications for early dromaeosaurid evolution. Proceedings of the Royal Society B: Biological Sciences, 277:211-217. https://doi.org/10.1098/rspb.2009.1178

Zhou, Z. and Li, F.Z.Z. 2009. A new Lower Cretaceous bird from China and tooth reduction in early avian evolution. Proceedings of the Royal Society of London B: Biological Sciences, rspb20090885. https://doi.org/10.1098/rspb.2009.0885

Zhou, Z.-H. and Wang, X.L. 2000. A new species of Caudipteryx from the Yixian Formation of Liaoning, northeast China. Vertebrata PalAsiatica, 38:113-130.

Zhou, Z.-H., Wang, X.-L., Zhang, F.-C., and Xu, X. 2000. Important features of Caudipteryxevidence from two nearly complete new specimens. Vertebrata PalAsiatica, 38:243-265.

Zinke, J. 1998. Small theropod teeth from the Upper Jurassic coal mine of Guimarota (Portugal). Paläontologische Zeitschrift, 72:179-189. https://doi.org/10.1007/bf02987825

Zinke, J. and Rauhut, O.W.M. 1994. Small theropods (Dinosauria, Saurischia) from the Upper Jurassic and Lower Cretaceous of the Iberian Peninsula. Berliner Geowissenschaftliche Abhandlugen (E), 13:163-177.

Zverkov, N.G., Fischer, V., Madzia, D., and Benson, R.B.J. 2018. Increased pliosaurid dental disparity across the Jurassic-Cretaceous transition. Palaeontology, 61:825-846. https:// doi.org/10.1111/pala.12367 


\section{APPENDICES}

The appendix files are available as PDFs at this url: https://palaeo-electronica.org/content/2019/ 2806-dental-features-in-theropods.

\section{APPENDIX 1.}

List of the 200 non-avian saurischian taxa included in this study and silhouette acknowledgements.

\section{APPENDIX 2.}

Dentition-based character list.

\section{APPENDIX 3.}

Phylogenetic character dataset.

\section{APPENDIX 4.}

$\mathrm{Cl}$ and $\mathrm{RI}$ values.

\section{APPENDIX 5.}

Distribution of all 145 apomorphic dental characters.

\section{APPENDIX 6.}

Distribution of the 34 dental character

\section{APPENDIX 7.}

Prior use and scorings of dental characters in the most recent phylogenetic analyses.

\section{APPENDIX 8.}

Zipped file containing spreadsheets are available at this url: https://palaeo-electronica.org/content/2019/2806-dental-features-in-theropods.

T-tests and Mann-Whitney U-tests using ci and ri values for each dental character. (ci_ri_dental characters.xlsx)

$\mathrm{Ci}$ and ri values for each dental character. (ci_ri_dental_characters_past3.xlsx)

Dentition-based datamatrix and dentition-based datamatrix combined with datasets on the whole skeleton. (teeth_characters_mesquite.xlsx) 